Resultados de multiplicidade para equações de Schrödinger com campo magnético via teoria de Morse e topologia do domínio 


\title{
Resultados de multiplicidade para equações de Schrödinger com campo magnético via teoria de Morse e topologia do domínio
}

\author{
Rodrigo Cohen Mota Nemer \\ Orientador: Prof. Dr. Sérgio Henrique Monari Soares \\ Coorientador: Prof. Dr. Claudianor Oliveira Alves
}

Tese apresentada ao Instituto de Ciências Matemáticas e de Computação - ICMC-USP, como parte dos requisitos para obtenção do título de Doutor em Ciências - Matemática. EXEMPLAR DE DEFESA. 
Ficha catalográfica elaborada pela Biblioteca Prof. Achille Bassi e Seção Técnica de Informática, ICMC/USP, com os dados fornecidos pelo(a) autor(a)

\begin{tabular}{|c|c|}
\hline \multirow[t]{3}{*}{ N433r } & $\begin{array}{l}\text { Nemer, Rodrigo Cohen Mota } \\
\text { Resultados de multiplicidade para equações de } \\
\text { Schrödinger com campo magnético via teoria de Morse } \\
\text { e topologia do domínio / Rodrigo Cohen Mota Nemer; } \\
\text { orientador Sérgio Henrique Monari Soares; co- } \\
\text { orientador Claudianor oliveira Alves. -- São } \\
\text { Carlos, } 2013 \text {. } \\
\quad 126 \text { p. }\end{array}$ \\
\hline & $\begin{array}{l}\text { Tese (Doutorado - Programa de Pós-Graduação em } \\
\text { Matemática) -- Instituto de Ciências Matemáticas e } \\
\text { de Computação, Universidade de São Paulo, } 2013 .\end{array}$ \\
\hline & $\begin{array}{l}\text { 1. Equações de Schrödinger. 2. Categoria de } \\
\text { Ljusternik-Schnirelman. 3. Teoria de Morse. I. } \\
\text { Soares, Sérgio Henrique Monari, orient. II. Alves, } \\
\text { Claudianor Oliveira, co-orient. III. Título. }\end{array}$ \\
\hline
\end{tabular}


Aos meus pais,

Jorge e Rosineide 



\section{Agradecimentos}

Aos meus pais, pelo amor, apoio e incentivo.

Aos ótimos amigos que aqui fiz, pela ajuda e pelos momentos de descontração.

Aos amigos de Santarém e de Campina Grande que, apesar da distância, mantiveram-se próximos e tornaram as visitas a essas cidades sempre prazerosas e revigorantes. Em especial, muito obrigado, Ciléia, por fazer parte da minha vida.

Aos professores Sérgio e Claudianor, meus orientadores, pelas conversas, paciência e incentivo, e sem os quais este trabalho não seria possível.

Aos professores do ICMC, em especial àqueles dos quais fui aluno, por todas as coisas que pude aprender.

Sei que esqueci de muita gente aqui, mas perdoem-me - se eu sou péssimo pra escrever matemática, imaginem pra escrever sobre coisas tão complicadas como sentimentos.

Por fim, à FAPESP pelo apoio financeiro. 

NEMER, R. C. M. Resultados de multiplicidade para equações de Schrödinger com campo magnético via teoria de Morse e topologia do domínio. 2013. 126 f. Tese (Doutorado) - Instituto de Ciências Matemáticas e de Computação, Universidade de São Paulo, São Carlos, 2013.

Neste trabalho, estudamos a existência de soluções não triviais para uma classe de equações de Schrödinger não lineares envolvendo um campo magnético com condição de Dirichlet ou condição de fronteira mista Dirichlet-Neumann. Nos dois primeiros capítulos, damos uma estimativa para o número de soluções não triviais para o problema de Dirichlet em termos da topologia do domínio. Nos dois capítulos restantes, consideramos o problema de fronteira mista e estimamos o número de soluções não triviais em termos da topologia da porção da fronteira onde é prescrita a condição de Neumann. Em ambos os casos, usamos a teoria de categoria de Ljusternik-Schnirelmann e a teoria de Morse.

Palavras-chave: Equações de Schrödinger não lineares. Métodos variacionais. Categoria de Ljusternik-Schnirelman. Teoria de Morse. 



\section{Abstract}

NEMER, R. C. M. Multiplicity results for nonlinear Schrödinger equations with magnetic field via Morse theory and domain topology. 2013. 126 f. Tese (Doutorado) - Instituto de Ciências Matemáticas e de Computação, Universidade de São Paulo, São Carlos, 2013.

We study the existence of nontrivial solutions for a class of nonlinear Schrödinger equations involving a magnetic field with Dirichlet or mixed Dirichlet-Neumann boundary condition. In the first two chapters we give an estimate for the number of nontrivial solutions for the Dirichlet boundary value problem in terms of topology of the domain. In the last two chapters we consider mixed Dirichlet-Neumann boundary value problems and the estimation of the number of nontrivial solutions is given in terms of the topology of the part of the boundary where the Neumann condition is prescribed. In both cases, we use LyusternikShnirelman category and the Morse theory.

Keywords: Nonlinear Schrödinger equations. Variational methods. Ljusternik-Schnirelman category. Morse theory. 



\section{Índice de Notações}

$B_{r}(x)$ é a bola de raio $r>0$ e centro $x \in \mathbb{R}^{N}$;

$\mathbb{R}_{+}:=\{x \in \mathbb{R} ; x \geq 0\}$;

$\mathbb{R}_{+}^{N}:=\left\{x=\left(x^{1}, x^{2}, \ldots, x^{N}\right) \in \mathbb{R}^{N} ; x^{N} \geq 0\right\} ;$

$\Omega-y:=\left\{x \in \mathbb{R}^{N} ; x=z-y\right.$, para algum $\left.z \in \Omega\right\}$;

$\operatorname{diam}(\Omega)$ é o diâmetro de $\Omega \subset \mathbb{R}^{N}$;

$\operatorname{dist}(X, Y)$ é a distância entre os conjuntos $X, Y \subset \mathbb{R}^{N}$;

$\frac{\partial u}{\partial \nu}$ denota a derivada normal exterior da função $u$;

$\operatorname{cat}_{X}(A)$ é a categoria de Ljusternik-Schnirelmann de $A$ em $X$ (ver página 2);

$\operatorname{cat}(A):=\operatorname{cat}_{A}(A)$

$|A|$ é a medida de Lebesgue de um subconjunto $A \subset \mathbb{R}^{N}$;

$L^{p}(\Omega, \mathbb{K})=\left\{u: \Omega \rightarrow \mathbb{K} ; u\right.$ é mensurável e $\left.\int_{\Omega}|u|^{p} \mathrm{~d} x<\infty\right\}$, onde $\mathbb{K}=\mathbb{R}$ ou $\mathbb{C}$;

$|u|_{p, \Omega}$ é a norma de $u \in L^{p}(\Omega, \mathbb{R})$;

$H^{1}(\Omega, \mathbb{K})=\left\{u \in L^{2}(\Omega, \mathbb{K}) ;|\nabla u| \in L^{2}(\Omega)\right\} ;$

$\|u\|_{\Omega}$ denota a norma de $H^{1}(\Omega, \mathbb{K})$;

$H_{0}^{1}(\Omega, \mathbb{R})=\overline{C_{0}^{\infty}(\Omega, \mathbb{R})}$, onde o fecho é tomado com relação a norma $\|\cdot\|_{\Omega}$;

$H_{0, \text { rad }}^{1}(\Omega, \mathbb{R})=\left\{u \in H_{0}^{1}(\Omega, \mathbb{R}) ; u\right.$ é radialmente simétrica $\} ;$

$\mathcal{M}\left(\mathbb{R}^{N}\right)$ é o espaço das medidas de Radon finitas em $\mathbb{R}^{N}$;

$\|\nu\|_{\mathcal{M}}:=\nu\left(\mathbb{R}^{N}\right)$ é a norma de $\nu \in \mathcal{M}\left(\mathbb{R}^{N}\right)$. 

Resumo $\quad$ v

Abstract vii

Índice de Notações $\quad$ ix

$\begin{array}{ll}\text { Introdução } & 1\end{array}$

1 Multiplicidade de soluções via teoria de Morse: problema de Dirichlet 9

1.1 Condição $(\mathrm{PS}) \ldots \ldots \ldots \ldots \ldots \ldots$

1.2 Comportamento dos níveis $\operatorname{minimax} \ldots \ldots \ldots \ldots$

1.3 Alguns resultados técnicos . . . . . . . . . . . . . . . . . . . . . . 24

1.4 Prova do Teorema $1.1 \ldots \ldots \ldots \ldots \ldots$

1.5 Provas dos Teorema 1.2 e Corolário $1.3 \ldots \ldots$. . . . . . . . . . 30

2 Multiplicidade de soluções via teoria de Morse: problema de Dirichlet homogêneo $\quad 33$

2.1 Condição $(\mathrm{PS}) \ldots \ldots \ldots \ldots \ldots \ldots \ldots \ldots$

2.2 Comportamento dos níveis $\operatorname{minimax} \ldots \ldots \ldots \ldots$

2.3 Preliminares . . . . . . . . . . . . . . . . . . . . . . . . . . . 41

2.4 A aplicação baricentro . . . . . . . . . . . . . . . . . . . . . 46

2.5 Prova do Teorema $2.1 \ldots \ldots \ldots \ldots \ldots$

2.6 Provas dos Teorema 2.2 e Corolário $2.3 \ldots \ldots \ldots$. . . . . . . . . 50

3 Equações de Schrödinger com condição de fronteira mista: caso homogêneo 53

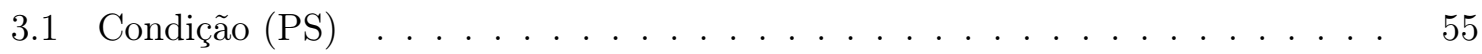




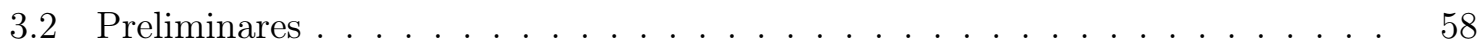

3.3 Comportamento dos níveis $\operatorname{minimax} \ldots \ldots \ldots$. . . . . . . . . . 64

3.4 A aplicação baricentro . . . . . . . . . . . . . . . . . . . . . . 77

3.5 Prova do Teorema $3.1 \ldots \ldots \ldots \ldots$. . . . . . . . . . . . . . 80

3.6 Provas dos Teorema 3.2 e Corolário $3.3 \ldots \ldots$. . . . . . . . . . . . . 81

4 Multiplicidade de soluções via teoria de Morse: condição de fronteira mistacaso geral $\quad 83$

4.1 Condição $(\mathrm{PS}) \ldots \ldots \ldots \ldots \ldots$. . . . . . . . . . . . . . . . . . . 85

4.2 Preliminares . . . . . . . . . . . . . . . . . . . . . . . . . . 88

4.3 Comportamento dos níveis minimax f . . . . . . . . . . . . . . . . . 90

4.4 A aplicação baricentro . . . . . . . . . . . . . . . . . . . . . . . 97

4.5 Prova do Teorema $4.1 \ldots \ldots \ldots$. . . . . . . . . . . . . . . 104

4.6 Provas dos Teorema 4.2 e Corolário $4.3 \ldots \ldots$. . . . . . . . . . . 105

$\begin{array}{ll}\text { A Teoria de Morse e resultados de homologia } & 107\end{array}$

A.1 Alguns resultados básicos de homologia . . . . . . . . . . . . . . . . . 107

A.2 Teoria de Morse para funcionais sobre espaços de Hilbert e o índice de Morse 108

A.2.1 Blocos de Conley . . . . . . . . . . . . . . . . . . . . . . . . . . 109

A.2.2 Índice de Morse generalizado . . . . . . . . . . . . . . . . . . . 111

A.3 Teoria de Morse e multiplicidade e soluções . . . . . . . . . . . . . . . . . . . 112

$\begin{array}{ll}\text { Referências Bibliográficas } & 123\end{array}$ 
A equação de Schrödinger não linear

$$
i h \frac{\partial \Psi}{\partial t}=\left(\frac{\hbar}{i} \nabla-A(x)\right)^{2} \Psi+U(x) \Psi-f\left(|\Psi|^{2}\right) \Psi, \quad x \in \Omega,
$$

tem um papel central em vários contextos físicos, tais como na física da matéria condensada e na ótica não linear [36]. O operador Schrödinger é definido por

$$
\left(\frac{\hbar}{i} \nabla-A(x)\right)^{2} \Psi=-\hbar^{2} \Delta \Psi-\frac{2 \hbar}{i} A \nabla \Psi+|A|^{2} \Psi-\frac{\hbar}{i} \Psi \operatorname{div} A,
$$

onde $A: \mathbb{R}^{N} \rightarrow \mathbb{R}^{N}$ é o potencial magnético e $U: \mathbb{R}^{N} \rightarrow \mathbb{R}^{N}$ é o potencial elétrico, $\Omega$ é domínio em $\mathbb{R}^{N}, t \in \mathbb{R}, \hbar$ é a constante de Planck, $i$ é a unidade imaginária, $\Psi: \mathbb{R} \times \mathbb{R}^{N} \rightarrow \mathbb{C}$ é a função de onda e $f$ é o termo não linear. O estudo do tipo especial de soluções da forma $\Psi(t, x)=e^{-i E t / h} u(x)$ para (1), quando $\hbar$ é suficientemente pequeno, leva a procurar por soluções $u: \Omega \rightarrow \mathbb{C}$ para a equação complexa em $\Omega$

$$
\left(\frac{\hbar}{i} \nabla-A(x)\right)^{2} u+V(x) u=f\left(|u|^{2}\right) u, \quad x \in \Omega
$$

sendo $V(x)=U(x)-E$. No caso particular em que $V \equiv 1$, vemos que $u$ é uma solução de (2) se, e só se, a função $v(x)=u(\hbar x)$ é solução para a equação

$$
\left(\frac{1}{i} \nabla-A_{\lambda}(x)\right)^{2} v+v=f\left(|v|^{2}\right) v, \quad x \in \Omega_{\lambda},
$$

onde $\lambda=h^{-1}, A_{\lambda}(x)=A\left(\lambda^{-1} x\right)$ e $\Omega_{\lambda} \doteq \lambda \Omega$.

O caso em que o campo magnético não está presente $(A=0)$ tem sido amplamente estudo na literatura, por exemplo, [4], [6], [10], [9], [23], [27], [32], [41], [40], e as demais referências citadas nesse trabalhos. Nos trabalhos pioneiros [10, 9], Benci e Cerami mostraram como a 
topologia de $\Omega$ afeta o número de soluções do problema

$$
\left\{\begin{array}{cl}
-\Delta u+\lambda u=u^{p-1}, & \text { em } \Omega, \\
u>0, & \text { em } \Omega, \\
u=0, & \text { sobre } \partial \Omega,
\end{array}\right.
$$

onde $\Omega \subset \mathbb{R}^{N}, N \geq 3$ é um domínio suave limitado, $p \in(2,2 N /(N-2))$ e $\lambda \geq 0$. Mais especificamente, eles usaram a categoria de Ljusternik-Schnirelman ${ }^{1}$ para mostrar que o número de soluções de (4) é pelo menos a categoria de $\Omega$, desde que $\lambda$ seja suficientemente grande (ou se $p \rightarrow 2^{*}=2 N /(N-2)$ ). Posteriormente, esses autores empregaram a teoria de Morse para estimar o número de soluções do problema

$$
\left\{\begin{array}{cl}
-\epsilon \Delta u+u=f(u), & \text { em } \Omega, \\
u>0, & \text { em } \Omega, \\
u=0, & \text { sobre } \partial \Omega,
\end{array}\right.
$$

em termos do polinômio de Poincaré ${ }^{2}$ de $\Omega, \mathcal{P}_{t}(\Omega)$, desde que $\epsilon>0$ seja suficientemente pequeno. É provado que para $\epsilon$ suficientemente pequeno, o número de soluções é pelo menos $\mathcal{P}_{t}(\Omega)$. Em [14], Candela e Lazzo consideraram a equação em (5) com condição de fronteira mista e $f(t)=|t|^{p-2} t$ e provaram que o número de soluções positivas é influenciado pela topologia da parte $\Gamma_{1}$ da fronteira $\partial \Omega$ onde a condição de Neumann é prescrita. Mais precisamente, se a medida de Lebesgue $(N-1)$-dimensional em $\mathbb{R}^{N}$ de $\Gamma_{1}$ é positiva, então o número de soluções do respectivo problema é pelo menos a categoria $\Gamma_{1}$, desde que $\epsilon$ seja suficientemente pequeno.

Resultados de existência para o caso magnético podem ser encontrados em [1], [3], [7], [15], [16], [17], [18], [19], [20], [21], [26], [28], [29], [30], [34], [37], [38], [39]. Em [3], os autores provaram que se $f$ é uma função superlinear com crescimento subcrítico, então para $\lambda>0$ suficientemente grande, o número de soluções não triviais do problema de Dirichlet para a equação (3) é pelo menos a categoria de $\Omega$.

Motivados pelos trabalhos citados, uma questão natural é se a teoria de Morse pode ser empregada para estimar o número de soluções para problemas não lineares com campo magnético. Esta tese tem por objetivo responder afirmativamente esta questão.

No Capítulo 1, estabelecemos que o campo magnético não desempenha papel algum sobre o número de soluções de (3) em $\Omega_{\lambda}$, e portanto um resultado no mesmo espírito de[9] é válido. Usando a teoria de Morse, obtemos uma estimativa do número de soluções do problema

$$
\begin{cases}\left(-i \nabla-A\left(\frac{x}{\lambda}\right)\right)^{2} u+u=f\left(|u|^{2}\right) u, & \text { em } \Omega_{\lambda}, \\ u=0, & \text { sobre } \partial \Omega_{\lambda},\end{cases}
$$

\footnotetext{
${ }^{1}$ Sejam $X$ um espaço topológico e $A$ um subconjunto fechado de $X$. A categoria de Ljusternik-Schnirelmann de $A$ em $X, \operatorname{cat}_{X}(A)$, é o menor natural $n$ tal que existem $n$ conjuntos fechados e contráteis (ver Definição A.3) em $X, A_{j} \subset A$, com $A=\bigcup_{j=1}^{n} A_{j}$.

${ }^{2}$ ver Definição A.5
} 
onde $\lambda$ é um parâmetro positivo, $\Omega_{\lambda}=\lambda \Omega, \Omega \subset \mathbb{R}^{N}$ é um domínio limitado, $N \geq 3$, $A \in C\left(\Omega, \mathbb{R}^{N}\right)$ and $f \in C^{1}(\mathbb{R})$ satisfaz:

$\left(f_{1}\right) \lim _{s \rightarrow 0} f(s)=0$ na origem;

$\left(f_{2}\right)$ existe $q \in\left(2,2^{*}\right)$ tal que

$$
\lim _{s \rightarrow \infty} \frac{f(s)}{s^{\frac{q-2}{2}}}=0 \text { e } \lim _{s \rightarrow \infty} \frac{f^{\prime}(s)}{s^{\frac{q-4}{2}}}=0
$$

onde $2^{*}=\frac{2 N}{N-2} ;$

$\left(f_{3}\right)$ existe $\theta>2$ tal que

$$
0<\frac{\theta}{2} F(s) \leq s f(s), \text { para } s>0,
$$

onde $F(s)=\int_{0}^{s} f(t) \mathrm{d} t$

$\left(f_{4}\right) f^{\prime}(s)>0$, para todo $s>0$.

Inspirados por [3] e [9], provaremos os seguintes resultados:

Teorema 0.1. Existe $\hat{\lambda}>0$ tal que, para $\lambda>\hat{\lambda}$, o problema $\left(P_{A_{\lambda}}\right)$ possui, pelo menos, $\operatorname{cat}\left(\Omega_{\lambda}\right)$ soluções.

Teorema 0.2. Seja $\hat{\lambda}>0$ dado no Teorema 0.1. Se $\lambda>\hat{\lambda}$ e o conjunto $\mathcal{K}$ de soluções de $\left(P_{A_{\lambda}}\right)$ é discreto, então

$$
\sum_{u \in \mathcal{K}} i_{t}(u)=t \mathcal{P}_{t}\left(\Omega_{\lambda}\right)+t^{2}\left[\mathcal{P}_{t}\left(\Omega_{\lambda}\right)-1\right]+(t+1) \mathcal{Q}(t)
$$

onde $\mathcal{Q}$ é um polinômio de coeficientes inteiros não-negativos, $\mathcal{P}_{t}\left(\Omega_{\lambda}\right)$ é o polinômio de Poincaré de $\Omega_{\lambda}$ e $i_{t}(u)$ é o indice de Morse generalizado ${ }^{3}$ de $u$.

No caso em que as soluções de $\left(P_{A_{\lambda}}\right)$ são não-degeneradas, temos o

Corolário 0.3. Seja $\hat{\lambda}>0$ dado no Teorema 0.1. Se $\lambda>\hat{\lambda}$ e as soluções de $\left(P_{A_{\lambda}}\right)$ são não-degeneradas, então

$$
\sum_{u \in \mathcal{K}} t^{m(u)}=t \mathcal{P}_{t}\left(\Omega_{\lambda}\right)+t^{2}\left[\mathcal{P}_{t}\left(\Omega_{\lambda}\right)-1\right]+(t+1) \mathcal{Q}(t)
$$

onde $\mathcal{Q}$ é um polinômio de coeficientes inteiros não-negativos e $m_{t}(u)$ é o índice de Morse ${ }^{4}$ de $u$.

Como observado em [9], e em [22], a aplicação da teoria de Morse pode fornecer informações melhores do o uso da categoria de Ljusternik-Schnirelman. O Corolário 0.3 mostra que $\left(P_{A, \kappa, p}\right)$ possui pelo menos $2 \mathcal{P}_{1}\left(\Omega_{\lambda}\right)-1$ soluções não triviais. No caso em que $\Omega$ é topologicamente trivial, segue que $\mathcal{P}_{1}\left(\Omega_{\lambda}\right)=1$ e este resultado não dá nehuma informação adicional sobre

\footnotetext{
${ }^{3}$ ver Definição A.14

${ }^{4}$ ver Definição A.7
} 
multiplicidade de soluções. Por outro lado, quando $\Omega$ é topologicamente rico, por exemplo, se $\Omega$ é obtido de um conjunto aberto contrátil do qual foi recortado $k$ conjuntos abertos abertos contráteis não vazios, obtemos que o número de soluções não triviais de $\left(P_{A_{\lambda}}\right)$ é afetado por $K$, mesmo que a categoria de $\Omega$ seja 2 . O Teorema 0.2 pode ser visto como uma versão do resultado de Alves, Figueiredo e Furtado em [3] em termos da teoria de Morse, cuja prova é baseada nas técnicas desenvolvidas em [9] e envolve os argumentos empregados em [3].

No Capítulo 2, retomamos o estudo do problema [10] no contexto de equações não lineares na presença do campo magnético. Estamos interessados em aplicar a teoria de Morse ao problema

$$
\left\{\begin{aligned}
(-i \nabla-A)^{2} u+\kappa u & =|u|^{p-2} u, \text { em } \Omega, \\
u & =0, \quad \text { sobre } \partial \Omega,
\end{aligned}\right.
$$

onde $\kappa$ é um parâmetro real positivo, $\Omega \subset \mathbb{R}^{N}$ é um domínio limitado, $N \geq 3$, $i$ é a unidade imaginária, $A: \Omega \rightarrow \mathbb{R}$ uma aplicação contínua e $p \in\left(2,2^{*}\right)$. Observamos que $\left(P_{A, \kappa, p}\right)$ pode ser escrito na forma do problema (2) fazendo $v(x)=(1 / \kappa)^{1 /(p-2)} u(x)$ e $\hbar=1 / \kappa$. Portanto, o método apresentado no Capítulo 1 se aplica ao problema $\left(P_{A, \kappa, p}\right)$; assim, se $\kappa$ é suficientemente grande, obtemos a mesma conclusão dos Teoremas 0.1 e 0.2 . Por outro lado, tal como em [10], provamos que para cada $\kappa$ fixo, se $p$ estiver próximo de $2^{*}$, podemos estimar o número de soluções do problema $\left(P_{A, \kappa, p}\right)$ em termos da teoria de Morse e da topologia do domínio. Mais especificamente, obtemos os seguintes resultados:

Teorema 0.4. Existe uma função $\bar{p}:[0,+\infty) \rightarrow\left(2,2^{*}\right)$ tal que para todo $p \in\left[\bar{p}(\kappa), 2^{*}\right)$, o problema $\left(P_{A, \kappa, p}\right)$ possui, pelo menos, cat $(\Omega)$ soluções não triviais.

Teorema 0.5. Seja $\bar{p}:[0,+\infty) \rightarrow\left(2,2^{*}\right)$ a função dada no Teorema 0.4. Suponha que o conjunto $\mathcal{K}$ de soluções do problema $\left(P_{A, \kappa, p}\right)$ é discreto. Então, para todo $p \in\left[\bar{p}(\kappa), 2^{*}\right)$,

$$
\sum_{u \in \mathcal{K}} i_{t}(u)=t \mathcal{P}_{t}(\Omega)+t^{2}\left[\mathcal{P}_{t}(\Omega)-1\right]+(t+1) \mathcal{Q}(t)
$$

onde $\mathcal{Q}$ é um polinômio com coeficientes inteiros não negativos.

No caso não degenerado, temos

Corolário 0.6. Seja $\bar{p}:[0,+\infty) \rightarrow\left(2,2^{*}\right)$ a função dada no Teorema 0.4. Suponha que as soluções de $\left(P_{A, \kappa, p}\right)$ sejam não degeneradas. Então, para todo $p \in\left[\bar{p}(\kappa), 2^{*}\right)$,

$$
\sum_{u \in \mathcal{K}} t^{m(u)}=t \mathcal{P}_{t}(\Omega)+t^{2}\left[\mathcal{P}_{t}(\Omega)-1\right]+(t+1) \mathcal{Q}(t)
$$

onde $\mathcal{Q}$ é um polinômio com coeficientes inteiros não negativos.

A partir do Corolário 0.6 , concluímos que $\left(P_{A, \kappa, p}\right)$ possui, pelo menos, $2 \mathcal{P}_{1}(\Omega)-1$ soluções não triviais. 
Os Capítulos 3 e 4 são dedicados ao estudo de multiplicidade de soluções para problemas com condição de fronteira mista da forma:

$$
\left\{\begin{aligned}
\left(-i \nabla-A_{\lambda}\right)^{2} u+u & =f\left(|u|^{2}\right) u, & & \text { em } \Omega_{\lambda}, \\
u & =0, & & \text { sobre } \Gamma_{0 \lambda}, \\
\frac{\partial u}{\partial \nu} & =0, & & \text { sobre } \Gamma_{1 \lambda},
\end{aligned}\right.
$$

onde $\lambda$ é um parâmetro real positivo, $\Omega_{\lambda}=\lambda \Omega$ é um domínio em expansão, $\Omega \subset \mathbb{R}^{N}(N \geq 3)$ é um domínio limitado e suave com fronteira $\partial \Omega=\overline{\Gamma_{0}} \cup \overline{\Gamma_{1}}$, sendo $\Gamma_{0}, \Gamma_{1}$ são subvariedades suaves disjuntas, a medida de Lebesgue $(N-1)$-dimensional de $\Gamma_{1}$ é positiva em $\mathbb{R}^{N}, \Gamma_{0 \lambda} \doteq \lambda \Gamma_{0}$, $\Gamma_{1 \lambda} \doteq \lambda \Gamma_{1}, A \in C\left(\Omega, \mathbb{R}^{N}\right)$. Quanto à não linearidade $f$, o Capítulo 3 é dedicado ao caso homogêneo $f(s)=f(1) s^{\frac{q-2}{2}}$, para $q \in\left(2,2^{*}\right)$, enquanto o Capítulo 4 trata o caso geral, a saber, $f \in C^{1}\left(\mathbb{R}^{+}\right)$satisfazendo, além das hipóteses $\left(f_{1}\right)-\left(f_{4}\right)$ já citadas,

$\left(f_{5}\right)$ Existem $q \in\left(2,2^{*}\right)$ e constante $C>0$ tais que

$$
s f(s)-F(s) \geq C|s|^{q / 2}, \text { para todo } s \geq 0 .
$$

Provamos que para o problema de fronteira mista, a condição de Neumann domina, no sentido que as soluções de energia minima se concentram em torno dos pontos de $\Gamma_{1}$ (a maior parte do suporte das soluções de energia mínima está concentrada nas proximidades de $\Gamma_{1}$ ); assim a multiplicidade de soluções é afetada pela topologia da parte da fronteira onde a condição de Neumann é prescrita. Mais precisamente, os resultados principais do Capítulo 4 são:

Teorema 0.7. Suponhamos que $f$ satisfaça $\left(f_{1}\right)-\left(f_{5}\right)$. Então existe $\lambda^{*}>0$ tal que para qualquer $\lambda>\lambda^{*}$ o problema $\left(P_{A_{\lambda}}\right)$ possui pelo menos cat $\left(\Gamma_{1 \lambda}\right)$ soluçôes não triviais.

Em termos da teoria de Morse, os resultados são:

Teorema 0.8. Suponhamos que $f$ satisfaça $\left(f_{1}\right)-\left(f_{5}\right)$ e que o conjunto $\mathcal{K}$ das soluções não triviais do problema $\left(P_{A_{\lambda}}\right)$ seja discreto. Então, existe $\lambda^{*}>0$ tal que

$$
\sum_{u \in \mathcal{K}} i_{t}(u)=t \mathcal{P}_{t}\left(\Gamma_{1 \lambda}\right)+t^{2}\left[\mathcal{P}_{t}\left(\Gamma_{1 \lambda}\right)-1\right]+(t+1) \mathcal{Q}(t)
$$

para qualquer $\lambda>\lambda^{*}$, onde $\mathcal{Q}(t)$ é um polinômio com coeficientes inteiros não negativos.

No caso degenerado, temos

Corolário 0.9. Suponhamos que $f$ satisfaça $\left(f_{1}\right)-\left(f_{5}\right)$ e que as soluções do problema $\left(P_{A_{\lambda}}\right)$ são não degeneradas. Então, existe $\lambda^{*}>0$ tal que

$$
\sum_{u \in \mathcal{K}} t^{\mu(u)}=t \mathcal{P}_{t}\left(\Gamma_{1 \lambda}\right)+t^{2}\left[\mathcal{P}_{t}\left(\Gamma_{1 \lambda}\right)-1\right]+(t+1) \mathcal{Q}(t)
$$

para qualquer $\lambda>\lambda^{*}$, onde $\mathcal{Q}(t)$ é um polinômio com coeficientes inteiros não negativos. 
Uma vez que o caso homogêneo $f(s)=f(1) s^{\frac{q-2}{2}}$ é um caso particular do caso geral, o método desenvolvido no Capítulo 4 pode ser aplicado ao respectivo problema obtendo a mesma conclusão dos Teoremas 0.1 e 0.2. No entanto, incluímos no Capítulo 3 uma demonstração independente desses resultados, pois o caso homogêneo permite fazer o uso de minimização em esferas de $L^{q}$ e, desta forma, estabelecer resultados de concentração como no Lema 3.14 via Lema de Concentração-Compacidade de Lions, tal qual em [42] e [14].

Como observado em [9, 22], a aplicação da teoria de Morse pode fornecer mais informações do que o uso do teorema de Ljusternik-Schnirelman. O Teorema 0.8 mostra que o problema $\left(P_{A_{\lambda}}\right)$ possui pelo menos $2 \mathcal{P}_{1}\left(\Gamma_{1 \lambda}\right)-1$ soluções não triviais. No caso em que $\Gamma_{1 \lambda}$ é topologicamente trivial, temos $\mathcal{P}_{1}\left(\Gamma_{1 \lambda}\right)=1$ e esse teorema não dá nehuma informação adicional sobre multiplicidade de soluções. Por outro lado, quando $\Gamma_{1 \lambda}$ é topologicamente rico, por exemplo se $\Gamma_{1 \lambda}$ é obtido de uma subvariedade da qual forma recortados $k$ conjuntos não vazios, abertos e contráteis em $\partial \Omega$, obtemos que o número de soluções não triviais é afetado por $k$, mesmo que a categoria de $\Gamma_{1 \lambda}$ seja 2 .

Para provarmos os Teoremas 0.7 and 0.8, combinamos o método de Benci e Cerami [9] com uma variação dos argumentos de Candela e Lazzo [14]. Os passos principais no método de Benci e Cerami são a análise do comportamento dos níveis críticos relacionados ao problema (5) e a comparação da topologia de $\Omega$ com alguns conjuntos de subnível do funcional associado com (5). Embora tenhamos usado este método, tivemos que fazer uma análise detalhada do comportamento do níveis minimax associados com o problema $\left(P_{A_{\lambda}}\right)$ e uma prova mais complexa de que a função baricentro aplica conjuntos apropriados de subnível do funcional associado com $\left(P_{A_{\lambda}}\right)$ restrito à variedade de Nehari em uma vizinhança da parte da fronteira de $\Omega$ onde a condição de Neumann é dada. Isto é porque a equação $\left(P_{A_{\lambda}}\right)$ envolve uma campo magnético e condição de fronteira mista. Em particular, nossa abordagem ao demonstrarmos este resultado deu-nos condições de aplicar tanto a teoria de categoria quanto a teoria de Morse à contagem de soluções, como em [9], não apenas no capítulo em questão, mas em todos os problemas desta tese. Além disso, como a não linearidade no Capítulo 4 não necessariamente é homogênea, nossos argumentos diferem aqueles daqueles utilizados em [14] por Candela e Lazzo. Uma vez que esses passos cruciais estão verificados, podemos empregar a teoria de Morse, bem como o teorema de Ljusternik-Schnirelman, para estimar o número de soluções não triviais para $\left(P_{A_{\lambda}}\right)$ em termos da topologia da parte da fronteira onde a condição de Neumann é prescrita. Os teoremas estabelecidos nesta tese podem ser vistos como um complemento dos estudos feitos em [3], [10], [9] e [14] nos seguintes aspectos: 1) Em [3] somente a condição de Dirichlet foi considerada e os resultados são dados em termos da categoria de LjusternikSchnirelman do domínio; 2) Em [10, 9], o problema foi considerado para o operador Laplaciano e condição de fronteira Dirichlet. Aqui lidamos com condição de fronteira mais geral e uma classe de operadores que incluem o Laplaciano como caso particular; 3) Em [14], o problema também foi considerado para o operador Laplaciano e com não linearidade homogênea. No Capítulo 4 lidamos com uma classe de não linearidades mais geral tendo as funções homogêneas de grau $\frac{q-2}{2}$ como caso particular. 
Por fim, acrescentamos um apêndice contendo um recorte, porém detalhado, da teoria de Morse utilizada neste trabalho. Acreditamos que com isso a leitura da tese será mais agradável e instrutiva ao leitor. 



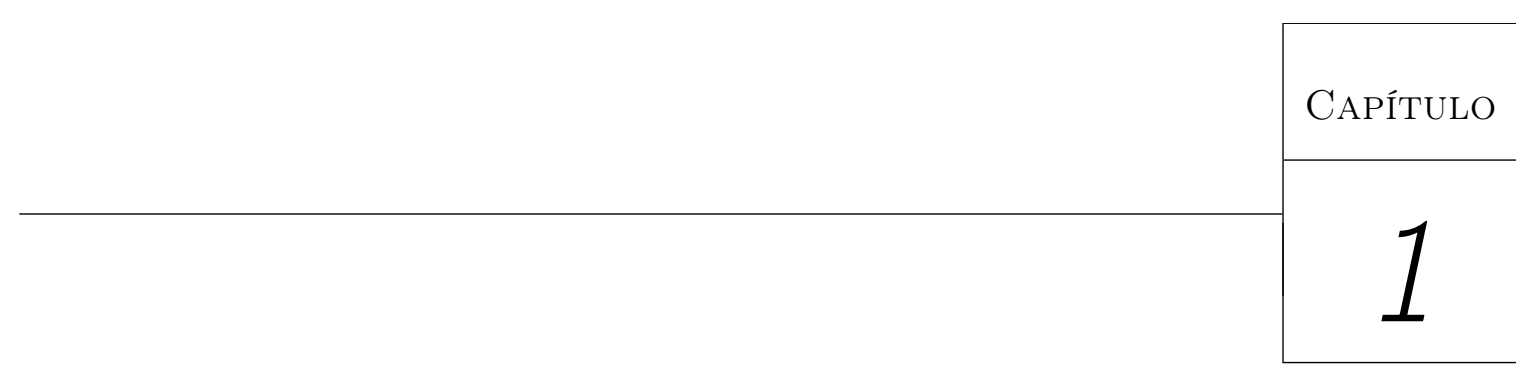

\section{Multiplicidade de soluções para} uma equação de Schrödinger envolvendo um campo magnético

Estamos interessados em aplicar a teoria de Morse ao problema

$$
\left\{\begin{aligned}
\left(-i \nabla-A_{\lambda}\right)^{2} u+u & =f\left(|u|^{2}\right) u, \quad \text { em } \Omega_{\lambda}, \\
u & =0, \quad \text { sobre } \partial \Omega_{\lambda},
\end{aligned}\right.
$$

onde $\Omega \subset \mathbb{R}^{N}$ é um domínio limitado, $N \geq 3, i$ é a unidade imaginária, $\lambda>0$ um parâmetro, $\Omega_{\lambda}:=\lambda \Omega$ um domínio em expansão, $A_{\lambda}:=A\left(\frac{x}{\lambda}\right), \operatorname{com} A: \Omega \rightarrow \mathbb{R}$ uma aplicação contínua e $f \in C^{1}\left(\mathbb{R}_{+}\right)$uma função satisfazendo algumas hipóteses, usando as técnicas apresentadas em Benci e Cerami [9].

A não linearidade $f$ satisfaz:

$\left(f_{1}\right) \lim _{s \rightarrow 0} f(s)=0$;

$\left(f_{2}\right)$ existe $q \in\left(2,2^{*}\right)$ tal que

$$
\lim _{s \rightarrow \infty} \frac{f(s)}{s^{\frac{q-2}{2}}}=0 \text { e } \lim _{s \rightarrow \infty} \frac{f^{\prime}(s)}{s^{\frac{q-4}{2}}}=0
$$


onde $2^{*}=\frac{2 N}{N-2} ;$

$\left(f_{3}\right)$ existe $\theta>2$ tal que

$$
0<\frac{\theta}{2} F(s) \leq s f(s), \text { para } s>0,
$$

onde $F(s)=\int_{0}^{s} f(t) \mathrm{d} t$;

$\left(f_{4}\right) f^{\prime}(s)>0$, para todo $s>0$

Como consequência de $\left(f_{1}\right)-\left(f_{2}\right)$,

$\left(f_{5}\right)$ dado $\epsilon>0$, existe $c_{\epsilon}>0$ tal que, para todo $s \in \mathbb{R}_{+}$,

$$
f(s) \leq \epsilon+c_{\epsilon}|s|^{\frac{q-2}{2}}, \quad F\left(s^{2}\right) \leq \epsilon s^{2}+c_{\epsilon}|s|^{q} .
$$

Por $\left(f_{3}\right)$, resulta

$\left(f_{6}\right)$ existem constantes $C_{1}, C_{2}>0$ tais que

$$
F\left(s^{2}\right) \geq C_{1}|s|^{\theta}-C_{2}, \forall s \geq 0,
$$

e, consequentemente, $\lim _{s \rightarrow \infty} f\left(s^{2}\right)=+\infty$.

Inspirados por [3] e [9], provaremos os seguintes resultados:

Teorema 1.1. Existe $\hat{\lambda}>0$ tal que, para $\lambda>\hat{\lambda}$, o problema $\left(P_{A_{\lambda}}\right)$ possui, pelo menos, $\operatorname{cat}\left(\Omega_{\lambda}\right)$ soluções.

Teorema 1.2. Seja $\hat{\lambda}>0$ dado no Teorema 1.1. Se $\lambda>\hat{\lambda}$ e o conjunto $\mathcal{K}$ de soluções de $\left(P_{A_{\lambda}}\right)$ é discreto, então

$$
\sum_{u \in \mathcal{K}} i_{t}(u)=t \mathcal{P}_{t}\left(\Omega_{\lambda}\right)+t^{2}\left[\mathcal{P}_{t}\left(\Omega_{\lambda}\right)-1\right]+(t+1) \mathcal{Q}(t)
$$

onde $\mathcal{Q}$ é um polinômio de coeficientes inteiros não-negativos, $\mathcal{P}_{t}\left(\Omega_{\lambda}\right)$ é o polinômio de Poincaré de $\Omega_{\lambda}$ e $i_{t}(u)$ é o indice de Morse generalizado de $u$.

No caso em que as soluções de $\left(P_{A_{\lambda}}\right)$ são não-degeneradas, temos o

Corolário 1.3. Seja $\hat{\lambda}>0$ dado no Teorema 1.1. Se $\lambda>\hat{\lambda}$ e as soluções de $\left(P_{A_{\lambda}}\right)$ são não-degeneradas, então

$$
\sum_{u \in \mathcal{K}} t^{m(u)}=t \mathcal{P}_{t}\left(\Omega_{\lambda}\right)+t^{2}\left[\mathcal{P}_{t}\left(\Omega_{\lambda}\right)-1\right]+(t+1) \mathcal{Q}(t)
$$

onde $\mathcal{Q}$ é um polinômio de coeficientes inteiros não-negativos, $\mathcal{P}_{t}\left(\Omega_{\lambda}\right)$ é o polinômio de Poincaré de $\Omega_{\lambda}$ e $m_{t}(u)$ é o indice de Morse de $u$.

A partir do Corolário 1.3, concluímos, em particular, que $\left(P_{A_{\lambda}}\right)$ possui, pelo menos, $2 \mathcal{P}_{1}\left(\Omega_{\lambda}\right)-1$ soluções não triviais. 


\subsection{Condição (PS)}

Para cada $\lambda>0$, denotemos por $E_{A_{\lambda}}$ o espaço de Hilbert sobre o corpo dos reais obtido como o fechamento de $C_{c}^{\infty}\left(\Omega_{\lambda}, \mathbb{C}\right)$ com a norma induzida pelo produto escalar

$$
<u, v>_{A_{\lambda}}:=\operatorname{Re}\left\{\int_{\Omega_{\lambda}}\left[\nabla_{A_{\lambda}} u \overline{\nabla_{A_{\lambda}} v}+u \bar{v}\right] \mathrm{d} x\right\},
$$

onde Rew é a parte real de $w \in \mathbb{C}, \bar{w}$ é o seu conjugado complexo, e

$$
\nabla_{A_{\lambda}} u:=\left(D_{A_{\lambda}}^{j} u\right)_{j=1}^{N}, \quad D_{A_{\lambda}}^{j} u=-i \partial_{j} u-A_{\lambda}^{j} u, \quad j \in\{1, \ldots, N\} .
$$

A norma induzida pelo produto interno acima é

$$
\|u\|_{A_{\lambda}}^{2}:=\int_{\Omega_{\lambda}}\left[\left|\nabla_{A_{\lambda}} u\right|^{2}+|u|^{2}\right] \mathrm{d} x .
$$

A seguir enunciaremos uma versão da desigualdade magnética presente em [26].

Lema 1.4. Vale

$$
\left|\nabla_{A_{\lambda}} u\right| \geq|\nabla| u||, \quad \forall u \in E_{A_{\lambda}} .
$$

Mais ainda, a imersão $E_{A_{\lambda}} \hookrightarrow L^{p}\left(\Omega_{\lambda}, \mathbb{R}\right)$ é compacta para cada $p \in\left[1,2^{*}\right)$ e é contínua para cada $p \in\left[1,2^{*}\right]$, com constantes de imersão independentes de $\lambda>0$, pela suavidade do conjunto $\Omega$ (ver [2]).

Diremos que $u \in E_{A_{\lambda}}$ é uma solução fraca do problema $\left(P_{\lambda}\right)$ se

$$
\operatorname{Re}\left(\int_{\Omega_{\lambda}}\left[\nabla_{A_{\lambda}} u \overline{\nabla_{A_{\lambda}} v}+u \bar{v}-f\left(|u|^{2}\right) u \bar{v}\right] \mathrm{d} x\right)=0, \forall v \in E_{A_{\lambda}} .
$$

Tendo em vista $\left(f_{1}\right),\left(f_{2}\right)$, o funcional $I_{\lambda}: E_{A_{\lambda}} \rightarrow \mathbb{R}$ dado por

$$
I_{\lambda}(u)=\frac{1}{2} \int_{\Omega_{\lambda}}\left[\left|\nabla_{A_{\lambda}} u\right|^{2}+|u|^{2}\right] \mathrm{d} x-\frac{1}{2} \int_{\Omega_{\lambda}} F\left(|u|^{2}\right) \mathrm{d} x, \forall u \in E_{A_{\lambda}},
$$

está bem definido. Mais ainda, $I_{\lambda} \in C^{2}\left(E_{A_{\lambda}}, \mathbb{R}\right)$ com

$$
I_{\lambda}^{\prime}(u) v=\operatorname{Re}\left(\int_{\Omega_{\lambda}}\left[\nabla_{A_{\lambda}} u \overline{\nabla_{A_{\lambda}} v}+u \bar{v}-f\left(|u|^{2}\right) u \bar{v}\right] \mathrm{d} x\right), \forall u, v \in E_{A_{\lambda}} .
$$

Assim, as soluções fracas de $\left(P_{\lambda}\right)$ são os pontos críticos de $I_{\lambda}$.

Proposição 1.5. O funcional $I_{\lambda}$ satisfaz a condição $(P S)_{c}$, para todo $c \in \mathbb{R}$, isto é, toda sequência $\left(u_{n}\right) \subset E_{A_{\lambda}}$ tal que $I_{\lambda}\left(u_{n}\right) \rightarrow c$ e $I_{\lambda}^{\prime}\left(u_{n}\right) \rightarrow 0$, quando $n \rightarrow \infty$, possui uma subsequência convergente.

\section{Demonstração.}


Sejam $c \in \mathbb{R}$ e $\left(u_{n}\right) \subset E_{A_{\lambda}}$ tais que $I_{\lambda}\left(u_{n}\right) \rightarrow c$ e $I_{\lambda}^{\prime}\left(u_{n}\right) \rightarrow 0$, quando $n \rightarrow \infty$. Então, por $\left(f_{3}\right)$, para $n$ suficientemente grande,

$$
\begin{aligned}
\left\|u_{n}\right\|_{A_{\lambda}}+c & \geq I_{\lambda}\left(u_{n}\right)-\frac{1}{\theta} I_{\lambda}^{\prime}\left(u_{n}\right) u_{n}= \\
& =\left(\frac{1}{2}-\frac{1}{\theta}\right)\left\|u_{n}\right\|_{A_{\lambda}}^{2}+\int_{\Omega_{\lambda}}\left(\frac{1}{\theta} f\left(\left|u_{n}\right|^{2}\right)\left|u_{n}\right|^{2}-\frac{1}{2} F\left(\left|u_{n}\right|^{2}\right)\right) \mathrm{d} x \geq \\
& \geq\left(\frac{1}{2}-\frac{1}{\theta}\right)\left\|u_{n}\right\|_{A_{\lambda}}^{2} .
\end{aligned}
$$

Logo $\left(u_{n}\right) \subset E_{A_{\lambda}}$ é limitada e existe $u \in E_{A_{\lambda}}$ tal que, a menos de subsequência,

$$
\left\{\begin{array}{l}
u_{n} \rightarrow u \text { em } E_{A_{\lambda}} \text { e } H_{0}^{1}\left(\Omega_{\lambda}\right), \\
u_{n} \rightarrow u \text { em } L^{p}\left(\Omega_{\lambda}\right), p \in\left[1,2^{*}\right), \\
u_{n}(x) \rightarrow u(x) \text { quase sempre em } \Omega_{\lambda}, \\
\left|u_{n}(x)\right| \leq h(x), \text { para alguma } h \in L^{q}\left(\Omega_{\lambda}\right),
\end{array}\right.
$$

quando $n \rightarrow \infty$. Notemos que o limite fraco $u \in E_{A_{\lambda}}$ é um ponto crítico de $I_{\lambda}$. De fato, por $(1.1),\left(f_{5}\right)$ e pelo Teorema da Convergência Dominada de Lebesgue, temos

$$
\begin{aligned}
\left\langle u_{n}, v\right\rangle_{A_{\lambda}} & \rightarrow\langle u, v\rangle_{A_{\lambda},} \\
\operatorname{Re}\left\{\int_{\Omega_{\lambda}} f\left(\left|u_{n}\right|^{2}\right) u_{n} \bar{v} \mathrm{~d} x\right\} & \rightarrow \operatorname{Re}\left\{\int_{\Omega_{\lambda}} f\left(|u|^{2}\right) u \bar{v} \mathrm{~d} x\right\},
\end{aligned}
$$

quando $n \rightarrow \infty$, donde

$$
I_{\lambda}^{\prime}(u) v=\lim _{n \rightarrow \infty} I_{\lambda}^{\prime}\left(u_{n}\right) v=0, \forall v \in E_{A_{\lambda}} .
$$

Por fim, mostremos que $\left(u_{n}\right)$ converge fortemente para $u$ em $E_{A_{\lambda}}$. Observemos que

$$
\begin{aligned}
\left\|u_{n}-u\right\|_{A_{\lambda}}^{2} & =\left(I_{\lambda}^{\prime}\left(u_{n}\right)-I_{\lambda}^{\prime}(u)\right)\left(u_{n}-u\right)+\operatorname{Re}\left\{\int_{\Omega_{\lambda}}\left(f\left(\left|u_{n}\right|^{2}\right) u_{n}-f\left(|u|^{2}\right) u\right) \overline{\left(u_{n}-u\right)} \mathrm{d} x\right\} \\
& \leq o_{n}(1)+\left|\int_{\Omega_{\lambda}}\left(f\left(\left|u_{n}\right|^{2}\right) u_{n}-f\left(|u|^{2}\right) u\right) \overline{\left(u_{n}-u\right)} \mathrm{d} x\right| \\
& \leq o_{n}(1)+\left|f\left(\left|u_{n}\right|^{2}\right) u_{n}-f\left(|u|^{2}\right) u\right|_{\frac{q}{q-1}, \Omega_{\lambda}}\left|u_{n}-u\right|_{q, \Omega_{\lambda} .}
\end{aligned}
$$

Por $\left(f_{5}\right), \sup _{n}\left|f\left(\left|u_{n}\right|^{2}\right) u_{n}-f\left(|u|^{2}\right) u\right|_{\frac{q}{q-1}, \Omega_{\lambda}}<\infty$ e, portanto, o resultado segue.

Teorema 1.6. Existe uma solução para o problema $\left(P_{A_{\lambda}}\right)$, para cada $\lambda>0$.

\section{Demonstração.}

Usaremos o Teorema do Passo da Montanha. Mostremos, então, que existem $\rho>0 \mathrm{e}$ $e \in E_{A_{\lambda}}$ tais que $\|e\|_{A_{\lambda}} \geq \rho$,

$$
I_{\lambda}(u) \geq 0 \text { em } B_{\rho}(0), \quad \inf _{u \in S_{\rho}(0)} I_{\lambda}(u) \geq \eta>0 \quad \text { e } \quad I_{\lambda}(e) \leq 0=I_{\lambda}(0) .
$$


Por $\left(f_{5}\right)$, para $\epsilon>0$ qualquer,

$$
I_{\lambda}(u) \geq \frac{1}{2}\|u\|_{A_{\lambda}}^{2}-\epsilon|u|_{2, \Omega_{\lambda}}^{2}-c_{\epsilon}|u|_{q, \Omega_{\lambda}}^{q},
$$

donde, usando as imersões $E_{A_{\lambda}} \hookrightarrow L^{p}\left(\Omega_{\lambda}\right), p \in\left[1,2^{*}\right)$, e tomando $\epsilon$ adequado,

$$
I_{\lambda}(u) \geq \frac{1}{4}\|u\|_{A_{\lambda}}^{2}-c\|u\|_{A_{\lambda}}^{q} .
$$

Como $q>2$, existe $\rho>0$ tal que $I_{\lambda}(u) \geq 0$ para $\|u\| \leq \rho$. Além disso, dada $\phi \in C_{c}^{\infty}\left(\Omega_{\lambda}\right)$, por $\left(f_{6}\right)$,

$$
\lim _{t \rightarrow \infty} I_{\lambda}(t \phi) \leq \lim _{t \rightarrow \infty} \frac{t^{2}}{2}\|\phi\|_{A_{\lambda}}^{2}-C_{1} t^{\theta} \int_{\Omega_{\lambda}}|\phi|^{\theta} \mathrm{d} x+C_{2}\left|\Omega_{\lambda}\right|=-\infty .
$$

Logo, para $t>0$ suficientemente grande, $e:=t \phi \in E_{A_{\lambda}}$ é tal que

$$
\|e\|>\rho \quad \text { e } \quad I_{\lambda}(e)<0 .
$$

Portanto, $I_{\lambda}$ possui a geometria do Passo da Montanha. Assim, pela Proposição 1.5 e pelo Teorema do Passo da Montanha de [5], existe solução para o problema $\left(P_{A_{\lambda}}\right)$.

Tendo em vista o resultado anterior, para cada $\lambda>0$, existe $u_{\lambda} \in E_{A_{\lambda}}$ tal que

$$
I_{\lambda}\left(u_{\lambda}\right)=b_{\lambda}, \quad I_{\lambda}^{\prime}\left(u_{\lambda}\right)=0,
$$

onde $b_{\lambda}$ é o nível do passo da montanha do funcional $I_{\lambda}$. Por $\left(f_{4}\right)$, argumentando como em [43, Theorem 4.2],

$$
b_{\lambda}=\inf _{u \in M_{\lambda}} I_{\lambda}(u)
$$

com

$$
M_{\lambda}:=\left\{u \in E_{A_{\lambda}} \backslash\{0\} ; I_{\lambda}^{\prime}(u) u=0\right\}=\left\{u \in E_{A_{\lambda}} \backslash\{0\} ;\|u\|_{A_{\lambda}}^{2}=\int_{\Omega_{\lambda}} f\left(|u|^{2}\right) u^{2} \mathrm{~d} x\right\}
$$

a variedade Nehari associada ao funcional. Temos, em relação ao conjunto $M_{\lambda}$, a seguinte estimativa.

Proposição 1.7. A variedade $M_{\lambda}$ é difeomorfa à esfera unitária em $E_{A_{\lambda}}$. Além disso, existe $\delta_{0}>0$ independente de $\lambda>0$ tal que, para todo $u \in M_{\lambda}$,

$$
\|u\|_{A_{\lambda}} \geq \delta_{0} \quad \text { e } \quad I_{\lambda}(u) \geq \delta_{0} .
$$

\section{Demonstração.}

Primeiramente verifiquemos a existência de $\delta_{0}>0$ satisfazendo as propriedades mencionadas.

Seja $u \in M_{\lambda}$ qualquer. Por $\left(f_{5}\right)$, temos, para qualquer $\epsilon>0$,

$$
\begin{aligned}
\|u\|_{A_{\lambda}}^{2} & =\int_{\Omega_{\lambda}} f\left(|u|^{2}\right)|u|^{2} \mathrm{~d} x \leq \epsilon|u|_{2, \Omega_{\lambda}}^{2}+c_{\epsilon}|u|_{q, \Omega_{\lambda}}^{q} \\
& \leq \epsilon C_{2}\|u\|_{A_{\lambda}}^{2}+c_{\epsilon} C_{q}\|u\|_{A_{\lambda}}^{q},
\end{aligned}
$$


onde $C_{p}$ é a constante de imersão $E_{A_{\lambda}} \hookrightarrow L^{p}\left(\Omega_{\lambda}, \mathbb{C}\right)$, a qual, pelo Lema 1.4, independe de $\lambda>0$. Como $q>2$, tomando $\epsilon>0$ suficientemente pequeno, temos

$$
\|u\|_{A_{\lambda}} \geq\left(\frac{1-\epsilon C_{2}}{c_{\epsilon} C_{q}}\right)^{\frac{1}{q-2}}=: \delta_{1}>0 .
$$

Note que, por $\left(f_{3}\right)$, se $u \in M_{\lambda}$,

$$
\|u\|_{A_{\lambda}}^{2}=\int_{\Omega_{\lambda}} f\left(|u|^{2}\right)|u|^{2} \mathrm{~d} x \geq \frac{\theta}{2} \int_{\Omega_{\lambda}} F\left(|u|^{2}\right) \mathrm{d} x .
$$

Assim, por (1.2) e (1.3),

$$
I_{\lambda}(u)=\frac{1}{2}\|u\|_{A_{\lambda}}^{2}-\frac{1}{2} \int_{\Omega_{\lambda}} F\left(|u|^{2}\right) \mathrm{d} x \geq\left(\frac{1}{2}-\frac{1}{\theta}\right)\|u\|_{A_{\lambda}}^{2} \geq\left(\frac{1}{2}-\frac{1}{\theta}\right) \delta_{1}=: \delta_{2} .
$$

Por fim, basta tomar $\delta_{0}:=\min \left\{\delta_{1}, \delta_{2}\right\}$.

Agora vejamos que $M_{\lambda}$ é difeomorfa à esfera unitária em $E_{A_{\lambda}}$, a qual denotaremos por $S_{1}^{A_{\lambda}}$. Consideremos a aplicação $\xi: S_{1}^{A_{\lambda}} \rightarrow(0,+\infty)$ que associa a cada $u \in S_{1}^{A_{\lambda}}$ o número $\xi(u)>0$.

$$
\frac{\mathrm{d}}{\mathrm{d} t} I_{\lambda}(\xi(u) u)=0
$$

Para vermos que $\xi$ está bem definida, observemos que

$$
\frac{\mathrm{d}}{\mathrm{d} t} I_{\lambda}(t u)=0 \Longleftrightarrow\|u\|_{A_{\lambda}}^{2}=\int_{\Omega_{\lambda}} f\left(t^{2}|u|^{2}\right)|u|^{2} \mathrm{~d} x .
$$

Logo, por $\left(f_{4}\right)$ e $\left(f_{6}\right)$, existe um único $\xi(u)>0$ tal que

$$
\|u\|_{A_{\lambda}}^{2}=\int_{\Omega_{\lambda}} f\left(\xi(u)^{2}|u|^{2}\right)|u|^{2} \mathrm{~d} x .
$$

Vejamos que $\xi$ é de classe $C^{1}$. Usaremos o Teorema da Função Implícita. Seja $\Psi(u, t)=\|u\|_{A_{\lambda}}^{2}-\int_{\Omega_{\lambda}} f\left(t^{2}|u|^{2}\right)|u|^{2}$, para $(u, t) \in(0+\infty) \times E_{A_{\lambda}}$. Seja também $\left(u_{0}, t_{0}\right) \in(0+\infty) \times E_{A_{\lambda}}$ tal que $\Psi\left(u_{0}, t_{0}\right)=0$. Então $t_{0} u_{0} \in M_{\lambda}$, isto é, $t_{0}>0$ e $u_{0} \not \equiv 0 \mathrm{em}$ um subconjunto de $\Omega_{\lambda}$ de medida positiva, já que $\left\|t_{0} u_{0}\right\|_{A_{\lambda}} \geq \delta_{0}$. Assim, tendo em vista $\left(f_{4}\right)$,

$$
\left.\frac{\partial \Psi(u, t)}{\partial t}\right|_{\left(u_{0}, t_{0}\right)}=-2 t_{0} \int_{\Omega_{\lambda}} f^{\prime}\left(t_{0}^{2}\left|u_{0}\right|^{2}\right)\left|u_{0}\right|^{4} \mathrm{~d} x<0 .
$$

Portanto, pelo Teorema da Função Implícita, $\xi$ é de classe $C^{1}$, uma vez que $f$ o é. Assim, $D: S_{1}^{A_{\lambda}} \rightarrow M_{\lambda}$ dada por

$$
D(u)=\xi(u) u \in M_{\lambda}
$$

é um difeomorfismo, cuja inversa é a restrição a $M_{\lambda}$ da projeção sobre a esfera $S_{1}^{A_{\lambda}}$.

Temos também 
Proposição 1.8. O funcional $I_{\lambda}$ restrito a $M_{\lambda}$ satisfaz a condição de $(P S)_{c}$, para todo $c \in \mathbb{R}$.

\section{Demonstração.}

Seja $\left(u_{n}\right)_{n} \subset M_{\lambda}$ uma sequência $(P S)_{c}$ para o funcional $I_{\lambda}$ restrito a $M_{\lambda}$, isto é,

$$
I_{\lambda}\left(u_{n}\right) \rightarrow c \in \mathbb{R} \text { e }\left(\left.I_{\lambda}\right|_{M_{\lambda}}\right)^{\prime}\left(u_{n}\right) \rightarrow 0, \text { quando } n \rightarrow \infty .
$$

Como em [43, Proposição 5.12], existe $\left(\mu_{n}\right) \subset \mathbb{R}$ tal que

$$
I_{\lambda}^{\prime}\left(u_{n}\right)=\mu_{n} G_{\lambda}^{\prime}\left(u_{n}\right)+o_{n}(1)
$$

onde $G_{\lambda}: E_{A_{\lambda}} \rightarrow \mathbb{R}$ é dada por

$$
G_{\lambda}(u)=I_{\lambda}^{\prime}(u) u=\int_{\Omega_{\lambda}}\left[\left|\nabla_{A_{\lambda}} u\right|^{2}+|u|^{2}\right] \mathrm{d} x-\int_{\Omega_{\lambda}} f\left(|u|^{2}\right)|u|^{2} \mathrm{~d} x .
$$

Como na Proposição 1.5, $\left(u_{n}\right)$ é limitada.

Como $u_{n} \in M_{\lambda}$, temos

$$
\begin{aligned}
G_{\lambda}^{\prime}\left(u_{n}\right) u_{n} & =2 \int_{\Omega_{\lambda}}\left[\left|\nabla_{A_{\lambda}} u_{n}\right|^{2}+\left|u_{n}\right|^{2}-f\left(\left|u_{n}\right|^{2}\right)\left|u_{n}\right|^{2}-f^{\prime}\left(\left|u_{n}\right|^{2}\right)\left|u_{n}\right|^{4}\right] \mathrm{d} x \\
& =-2 \int_{\Omega_{\lambda}} f^{\prime}\left(\left|u_{n}\right|^{2}\right)\left|u_{n}\right|^{4} \mathrm{~d} x .
\end{aligned}
$$

Além disso, pela Proposição 1.7, existe $\delta_{0}>0$ tal que

$$
\left\|u_{n}\right\|_{A_{\lambda}} \geq \delta_{0}, \quad \forall n \in \mathbb{N}
$$

ou seja, como $u_{n} \in M_{\lambda}$,

$$
\int_{\Omega_{\lambda}} f\left(\left|u_{n}\right|^{2}\right)\left|u_{n}\right|^{2} \mathrm{~d} x \geq \delta_{0}, \quad \forall n \in \mathbb{N} .
$$

Por (1.6) e $\left(f_{5}\right)$, segue do Lema de Lions (ver [43, Lema 1.21]), que existem $\left(y_{n}\right) \subset \Omega_{\lambda}, l>0$ e $\gamma>0$ tais que

$$
\liminf _{n \rightarrow \infty} \int_{\Omega_{\lambda} \cap B_{l}\left(y_{n}\right)}\left|u_{n}\right|^{p} \mathrm{~d} x \geq \gamma>0, \quad \forall p \in\left[1,2^{*}\right) .
$$

Notemos que, a menos de subsequência, $y_{n} \rightarrow y \in \overline{\Omega_{\lambda}}$. Assim, aumentando $l$ se necessário, podemos reescrever (1.7) como

$$
\liminf _{n \rightarrow \infty} \int_{\Omega_{\lambda} \cap B_{l}(y)}\left|u_{n}\right|^{p} \mathrm{~d} x \geq \gamma>0, \quad \forall p \in\left[1,2^{*}\right) .
$$

Seja, para cada $n \in \mathbb{N}$,

$$
\tilde{u}_{n}(x):=u_{n}(x+y), \quad \forall x \in \Omega_{\lambda}-y
$$


$\operatorname{Então}\left(\tilde{u}_{n}\right) \subset E_{A_{\lambda}(.+y)}:={\overline{C_{c}^{\infty}\left(\Omega_{\lambda}-y_{\lambda}, \mathbb{C}\right)}}^{\|\cdot\|_{A_{\lambda}\left(\cdot+y_{\lambda}\right)}}$, completamento de $C_{c}^{\infty}\left(\Omega_{\lambda}-y_{\lambda}, \mathbb{C}\right)$ relativamente à norma $\|\cdot\|_{A_{\lambda}\left(.+y_{\lambda}\right)}$, é uma sequência limitada, uma vez que $\left(u_{n}\right)$ o é. Logo existe $\tilde{u} \in E_{A_{\lambda}(.+y)}$ tal que valem, a menos de subsequência, os limites

$$
\left\{\begin{array}{l}
\tilde{u}_{n} \rightarrow \tilde{u} \text { em } E_{A_{\lambda}(.+y)}, \\
\tilde{u}_{n} \rightarrow \tilde{u} \text { em } L^{p}\left(\Omega_{\lambda}-y, \mathbb{C}\right), \forall p \in\left[1,2^{*}\right), \\
\tilde{u}_{n} \rightarrow \tilde{u} \text { quase sempre em } \Omega_{\lambda}-y .
\end{array}\right.
$$

Portanto, por (1.8),

$$
\int_{\Omega_{\lambda}-y}|\tilde{u}|^{2} \geq \gamma>0
$$

isto é, $\tilde{u} \not \equiv 0$.

Agora, por (1.5), (1.9) e $\left(f_{4}\right)$, aplicando o Lema de Fatou,

$$
\begin{aligned}
\liminf _{n \rightarrow \infty} G_{\lambda}^{\prime}\left(u_{n}\right) u_{n} & =-2 \liminf _{n \rightarrow \infty} \int_{\Omega_{\lambda}} f^{\prime}\left(\left|u_{n}\right|^{2}\right)\left|u_{n}\right|^{4} \mathrm{~d} x \\
& =-2 \liminf _{n \rightarrow \infty} \int_{\Omega_{\lambda}-y} f^{\prime}\left(\left|\tilde{u}_{n}\right|^{2}\right)\left|\tilde{u}_{n}\right|^{4} \mathrm{~d} x \\
& \leq-2 \int_{\Omega_{\lambda}-y} f^{\prime}\left(|\tilde{u}|^{2}\right)|\tilde{u}|^{4} \mathrm{~d} x<0 .
\end{aligned}
$$

Dessa forma, aplicando $u_{n}$ a ambos os membros de (1.4), obtemos $\mu_{n}=o_{n}(1)$. Logo $I_{\lambda}\left(u_{n}\right) \rightarrow 0$ e $I_{\lambda}^{\prime}\left(u_{n}\right) \rightarrow 0$ quando $n \rightarrow+\infty$. O resultado segue da Proposição 1.5.

Seguindo o raciocínio da demonstração anterior, provamos o seguinte resultado:

Corolário 1.9. Todo ponto crítico de $\left.I_{\lambda}\right|_{M_{\lambda}}$ é um ponto crítico de $I_{\lambda}$.

Seja $J_{\infty}: H^{1}\left(\mathbb{R}^{N}, \mathbb{R}\right) \rightarrow \mathbb{R}$ dado por

$$
J_{\infty}(u)=\frac{1}{2} \int_{\mathbb{R}^{N}}\left[|\nabla u|^{2}+u^{2}\right] \mathrm{d} x-\int_{\mathbb{R}^{N}} F\left(u^{2}\right) \mathrm{d} x,
$$

com variedade Nehari e nível do passo da montanha

$$
N_{\infty}=\left\{u \in H^{1}\left(\mathbb{R}^{N}, \mathbb{R}\right) \backslash\{0\} ; J_{\infty}^{\prime}(u) u=0\right\}, \quad c_{\infty}=\inf _{u \in N_{\infty}} J_{\infty}(u) .
$$

Vejamos um resultado de compacidade associado ao funcional $J_{\infty}$.

Proposição 1.10. Seja $\left(v_{n}\right) \subset N_{\infty}$ tal que $J_{\infty}\left(v_{n}\right) \rightarrow c_{\infty}$ quando $n \rightarrow \infty$. Então

(i) $v \not \equiv 0$ e $v_{n} \rightarrow v$ em $H^{1}\left(\mathbb{R}^{N}, \mathbb{R}\right), \operatorname{com} J_{\infty}(v)=c_{\infty}$ e $J_{\infty}^{\prime}(v)=0$;

(ii) existe $\left(y_{n}\right) \subset \mathbb{R}^{N}$, com $\left|y_{n}\right| \rightarrow \infty$, tal que a sequência $\tilde{v}_{n}:=v_{n}\left(.-y_{n}\right)$ converge fortemente para $\tilde{v} \not \equiv 0$ em $H^{1}\left(\mathbb{R}^{N}, \mathbb{R}\right)$. Mais ainda, $J_{\infty}(\tilde{v})=c_{\infty}$ e $J_{\infty}^{\prime}(\tilde{v})=0$ 


\section{Demonstração.}

Primeiramente, observe que $\left(v_{n}\right)$ é limitada em $H^{1}\left(\mathbb{R}^{N}, \mathbb{R}\right)$, como na demonstração da Proposição 1.5. Portanto, a menos de subsequência,

$$
v_{n} \rightarrow v \text { em } H^{1}\left(\mathbb{R}^{N}, \mathbb{R}\right) \text { e } v_{n} \rightarrow v \text { quase sempre em } \mathbb{R}^{N} .
$$

Sem perda de generalidade, podemos supor que $\left(v_{n}\right)$ é tal que

$$
J_{\infty}\left(v_{n}\right) \rightarrow c_{\infty}, J_{\infty}^{\prime}\left(v_{n}\right) \rightarrow 0
$$

Com efeito, pelo Princípio Variacional de Ekeland, existe $\left(u_{n}\right) \subset N_{\infty}$ tal que $u_{n}:=v_{n}+o_{n}(1)$

$$
J_{\infty}\left(u_{n}\right) \rightarrow c_{\infty} \text { e } J_{\infty}^{\prime}\left(u_{n}\right)-\gamma_{n} E_{\infty}^{\prime}\left(u_{n}\right)=\left(\left.J_{\infty}\right|_{N_{\infty}}\right)^{\prime}\left(u_{n}\right)=o_{n}(1)
$$

onde $\left(\gamma_{n}\right) \subset \mathbb{R}$ e $E_{\infty}(u):=J_{\infty}^{\prime}(u) u$, para todo $u \in H^{1}\left(\mathbb{R}^{N}, \mathbb{R}\right)$. Seguindo os argumentos da Proposição 1.8,

$$
\gamma_{n}=o_{n}(1)
$$

e daí $\left(u_{n}\right)$ é tal que

$$
J_{\infty}\left(u_{n}\right) \rightarrow c_{\infty}, J_{\infty}^{\prime}\left(u_{n}\right) \rightarrow 0 .
$$

Sem perda de generalidade, podemos tomar $\left(v_{n}\right)$ satisfazendo (1.11).

Temos dois casos a considerar:

(1) $v \not \equiv 0$;

(2) $v \equiv 0$.

Suponhamos que (1) ocorra. Mostremos $v \in N_{\infty}$. Usaremos o fato de $J_{\infty}^{\prime}\left(v_{n}\right) \rightarrow 0$ quando $n \rightarrow \infty$.

Primeiramente notemos que dado $\delta>0$ qualquer, existe $R_{\delta}>0$ tal que

$$
\left|\int_{B_{R_{\delta}} c(0)} f\left(v_{n}^{2}\right) v_{n} v \mathrm{~d} x\right| \leq C \delta
$$

uniformemente em $n \in \mathbb{N}$, onde $C>0$ é uma constante e $B_{R_{\delta}}^{c}(0):=\mathbb{R}^{N} \backslash B_{R_{\delta}}(0)$. Com efeito, pelas imersões de Sobolev, $v \in L^{2}\left(\mathbb{R}^{N}\right) \cap L^{q}\left(\mathbb{R}^{N}\right)$, donde existe $R_{\delta}>0$ tal que

$$
|v|_{2, B_{R_{\delta}}^{c}(0)},|v|_{q, B_{R_{\delta}}^{c}(0)} \leq \delta
$$

Usando $\left(f_{5}\right) \operatorname{com} \epsilon=1$ e a desigualdade de Hölder, obtemos

$$
\begin{aligned}
\left|\int_{B_{R_{\delta}}^{c}(0)} f\left(v_{n}^{2}\right) v_{n} v \mathrm{~d} x\right| & \leq \int_{B_{R_{\delta}}^{c}(0)}\left|v_{n} v\right| \mathrm{d} x+c_{1} \int_{B_{R_{\delta}}^{c}(0)}\left|v_{n}^{q-1} v\right| \mathrm{d} x \\
& \leq\left|v_{n}\right|_{2, B_{R_{\delta}}^{c}(0)}|v|_{2, B_{R_{\delta}}^{c}(0)}+c_{1}\left|v_{n}\right|_{\frac{q}{q-1}, B_{R_{\delta}}^{c}(0)}|v|_{q, B_{R_{\delta}}^{c}(0)} .
\end{aligned}
$$


Disto, juntamente com a limitação de $\left(v_{n}\right)_{n} \subset H^{1}\left(\mathbb{R}^{N}, \mathbb{R}\right)$, segue (1.13).

Temos $f\left(v_{n}^{2}\right) v_{n} \rightarrow f\left(v^{2}\right) v$ em $L^{\frac{q}{q-1}}\left(B_{R_{\delta}}(0)\right)$, pois, por $\left(f_{5}\right), \sup _{n}\left|f\left(v_{n}^{2}\right) v_{n}\right|_{\frac{q}{q-1}, B_{R_{\delta}}(0)}<\infty \mathrm{e}$ $f\left(v_{n}^{2}(x)\right) v_{n}(x) \rightarrow f\left(v^{2}(x)\right) v(x)$ quase sempre em $\mathbb{R}^{N}$. Dessa forma, usando (1.10) e (1.13), quando $n \rightarrow \infty$,

$$
\begin{aligned}
\left|\int_{\mathbb{R}^{N}}\left[f\left(v_{n}^{2}\right) v_{n} v-f\left(v^{2}\right) v^{2}\right] \mathrm{d} x\right| & \leq\left(\int_{B_{R_{\delta}}(0)}+\int_{B_{R_{\delta}}^{c}(0)}\right)\left|f\left(v_{n}^{2}\right) v_{n} v-f\left(v^{2}\right) v^{2}\right| \mathrm{d} x \\
& \leq o_{n}(1)+C \delta,
\end{aligned}
$$

para algum $C>0$, aumentando $R_{\delta}$ se necessário. Logo, como $\delta>0$ é qualquer, segue que $f\left(v_{n}^{2}\right) v_{n} v \rightarrow f\left(v^{2}\right) v^{2}$ em $L^{1}\left(\mathbb{R}^{N}\right)$ quando $n \rightarrow \infty$. Por fim, pela convergência fraca de $\left(v_{n}\right)_{n}$ e pelo limite anterior, temos, quando $n \rightarrow \infty$,

$$
\begin{aligned}
o_{n}(1) & =J_{\infty}^{\prime}\left(v_{n}\right) v=\int_{\mathbb{R}^{N}}\left(\nabla v_{n} \nabla v+v_{n} v\right) \mathrm{d} x-\int_{\mathbb{R}^{N}} f\left(v_{n}^{2}\right) v^{2} \mathrm{~d} x \\
& \rightarrow \int_{\mathbb{R}^{N}}\left(|\nabla v|^{2}+v^{2}\right) \mathrm{d} x-\int_{\mathbb{R}^{N}} f\left(v^{2}\right) v^{2} \mathrm{~d} x \\
& =J_{\infty}^{\prime}(v) v
\end{aligned}
$$

e, pela unicidade do limite, $v \in N_{\infty}$.

Agora, de $v \in N_{\infty}$, do Lema de Fatou e $\left(f_{3}\right)$,

$$
\begin{aligned}
c_{\infty} & \leq J_{\infty}(v)=\frac{1}{2} \int_{\mathbb{R}^{N}}\left(f\left(v^{2}\right) v^{2}-F\left(v^{2}\right)\right) \mathrm{d} x \\
& \leq \liminf _{n \rightarrow \infty} \frac{1}{2} \int_{\mathbb{R}^{N}}\left(f\left(v_{n}^{2}\right) v_{n}^{2}-F\left(v_{n}^{2}\right)\right) \mathrm{d} x \\
& \leq \limsup _{n \rightarrow \infty} \frac{1}{2} \int_{\mathbb{R}^{N}}\left(f\left(v_{n}^{2}\right) v_{n}^{2}-F\left(v_{n}^{2}\right)\right) \mathrm{d} x \\
& =c_{\infty} .
\end{aligned}
$$

Portanto, quando $n \rightarrow \infty$,

$$
\left\|v_{n}\right\|^{2}=\int_{\mathbb{R}^{N}}\left(f\left(v_{n}^{2}\right) v_{n}^{2}-F\left(v_{n}^{2}\right)\right) \mathrm{d} x \rightarrow \int_{\mathbb{R}^{N}}\left(f\left(v^{2}\right) v^{2}-F\left(v^{2}\right)\right) \mathrm{d} x=\|v\|^{2} .
$$

Assim

$$
v_{n} \rightarrow v \text { em } H^{1}\left(\mathbb{R}^{N}, \mathbb{R}\right),
$$

uma vez que $v_{n} \rightarrow v$ em $H^{1}\left(\mathbb{R}^{N}, \mathbb{R}\right)$, o qual é um espaço uniformemente convexo.

(2) $v \equiv 0$

Suponhamos agora que o caso (2) ocorra. Se $v \equiv 0$, então exitem $R, \eta>0$ e $\left(y_{n}\right) \subset \mathbb{R}^{N}$ tais que

$$
\limsup _{n \rightarrow \infty} \int_{B_{R}\left(y_{n}\right)}\left|v_{n}\right|^{2} \mathrm{~d} x \geq \eta .
$$

Com efeito, caso

$$
\lim _{n \rightarrow \infty} \sup _{y \in \mathbb{R}^{N}} \int_{B_{R}(y)}\left|v_{n}\right|^{2} \mathrm{~d} x=0,
$$


então pelo Lema de Lions, [43, Lema 1.21],

$$
\lim _{n \rightarrow \infty} \int_{\mathbb{R}^{N}}\left|v_{n}\right|^{s} \mathrm{~d} x=0, \forall s \in\left(2,2^{*}\right),
$$

donde, por $\left(f_{5}\right)$ e de $\left(v_{n}\right) \subset N_{\infty}$,

$$
\left\|v_{n}\right\|^{2}=\int_{\mathbb{R}^{N}} f\left(v_{n}^{2}\right) v_{n}^{2} \mathrm{~d} x \rightarrow 0, \quad \text { quando } n \rightarrow \infty .
$$

Logo, pela continuidade de $J_{\infty}, J_{\infty}\left(v_{n}\right) \rightarrow 0$, quando $n \rightarrow \infty$, o que contradiz o fato de $\left(v_{n}\right)$ ser uma sequência $(P S)_{c_{\infty}}$ e $c_{\infty}>0$.

Das imersões de Sobolev, $\left|y_{n}\right| \rightarrow \infty$. Com efeito, se $\left(y_{n}\right)$ é limitada, então, tomando $\bar{R}>0$ tal que $\bar{R}>\max _{n \in \mathbb{N}}\left|y_{n}\right|+R$,

$$
\eta \leq \lim _{n \rightarrow+\infty} \int_{B_{\bar{R}}}\left|v_{n}\right|^{2} \mathrm{~d} x=\int_{B_{\bar{R}}}|v|^{2} \mathrm{~d} x
$$

o que contradiz o fato de $v \equiv 0$.

Seja

$$
w_{n}(x):=v_{n}\left(x+y_{n}\right), \forall n \in \mathbb{N}, x \in \mathbb{R}^{N} .
$$

Então

$$
J_{\infty}\left(w_{n}\right) \rightarrow c_{\infty} \text { e } J_{\infty}^{\prime}\left(w_{n}\right) \rightarrow 0 .
$$

$\operatorname{Logo}\left(w_{n}\right) \subset H^{1}\left(\mathbb{R}^{N}, \mathbb{R}\right)$ é limitada e existe $w \in H^{1}\left(\mathbb{R}^{N}, \mathbb{R}\right)$ tal que

$$
w_{n} \rightarrow w \operatorname{em~} H^{1}\left(\mathbb{R}^{N}, \mathbb{R}\right) .
$$

Como no caso anterior, $w_{n} \rightarrow w$ em $H^{1}\left(\mathbb{R}^{N}, \mathbb{R}\right)$ e, assim, $J_{\infty}(w)=c_{\infty}$ e $J_{\infty}^{\prime}(w)=0$.

\subsection{Comportamento dos níveis minimax}

Considere agora o funcional $J_{\lambda}: H_{0}^{1}\left(\Omega_{\lambda}, \mathbb{R}\right)$ dado por

$$
J_{\lambda}(v)=\frac{1}{2} \int_{\Omega_{\lambda}}\left(|\nabla v|^{2}+v^{2}\right) \mathrm{d} x-\frac{1}{2} \int_{\Omega_{\lambda}} F\left(v^{2}\right) \mathrm{d} x .
$$

Definamos também

$$
a_{\lambda}:=\inf _{N_{\lambda}} J_{\lambda}
$$

onde

$$
N_{\lambda}:=\left\{v \in H_{0}^{1}\left(\Omega_{\lambda}, \mathbb{R}\right) \backslash\{0\} ; J_{\lambda}^{\prime}(v) v=0\right\}
$$

é a variedade Nehari associada ao funcional $J_{\lambda}$. 
Suporemos, sem perder a generalidade, que $0 \in \Omega$. Fixemos $r>0$ tal que $B_{\lambda r}(0) \subset \Omega_{\lambda}$. Definimos de forma análoga as ternas $\left(I_{\lambda, r}, b_{\lambda, r}, M_{\lambda, r}\right)$ e $\left(J_{\lambda, r}, c_{\lambda, r}, N_{\lambda, r}\right)$ apenas trocando $\Omega_{\lambda}$ por $B_{\lambda r}(0)$ nas definições de $\left(I_{\lambda}, b_{\lambda}, M_{\lambda}\right)$ e $\left(J_{\lambda}, c_{\lambda}, N_{\lambda}\right)$, respectivamente.

Vale ressaltar que todos os funcionais definidos acima e suas restrições às suas variedades Nehari satisfazem a condição $(P S)_{c}$, para todo $c \in \mathbb{R}$. Para ver isto, basta argumentar como nas Proposições 1.5 e 1.8.

Lema 1.11. Suponhamos que $f$ satisfaça $\left(f_{1}\right)-\left(f_{4}\right)$. Então

$$
\lim _{\lambda \rightarrow \infty} c_{\lambda, r}=c_{\infty}
$$

\section{Demonstração.}

Seja $\phi \in C_{c}^{\infty}\left(\mathbb{R}^{N}\right)$ tal que

$$
\phi(x)=1, \forall x \in B_{\frac{1}{2}}(0), \quad \operatorname{supp} \phi \subset \subset B_{1}(0), \quad 0 \leq \phi(x) \leq 1, \forall x \in \mathbb{R}^{N} .
$$

Observe que, pela definição de $c_{\infty}$ e pela Proposição 1.10 , existe $v \in H^{1}\left(\mathbb{R}^{N}, \mathbb{R}\right)$ tal que

$$
J_{\infty}(v)=c_{\infty}, J_{\infty}^{\prime}(v)=0 .
$$

Para cada $\lambda>0$, considere

$$
\phi_{\lambda}(x):=\phi\left(\frac{x}{\lambda r}\right) \text { e } v_{\lambda}(x):=v(x) \phi_{\lambda}(x) .
$$

Por $\left(f_{1}\right),\left(f_{4}\right)$ e $\left(f_{6}\right)$, para cada $\lambda>0$, existe único $t_{\lambda}>0$ tal que $t_{\lambda} v_{\lambda} \in N_{\lambda, r}$. Daí

$$
c_{\lambda, r} \leq J_{\lambda, r}\left(t_{\lambda} v_{\lambda}\right)=J_{\infty}\left(t_{\lambda} v_{\lambda}\right), \quad \forall \lambda>0
$$

Como $v \in N_{\infty}$, temos

$$
\lim _{\lambda \rightarrow \infty} t_{\lambda}=1
$$

Com efeito, pela definição de $t_{\lambda}$,

$$
\int_{\mathbb{R}^{N}}\left(\left|\nabla v_{\lambda}\right|^{2}+v_{\lambda}^{2}\right) \mathrm{d} x=\int_{\mathbb{R}^{N}} f\left(t_{\lambda}^{2} v_{\lambda}^{2}\right) v_{\lambda}^{2} \mathrm{~d} x
$$

Note que $\left(t_{\lambda}\right)$ é limitada. De fato, se, a menos de subsequência, $t_{\lambda} \rightarrow \infty$ quando $\lambda \rightarrow \infty$, então, pelo Lema de Fatou e $\left(f_{6}\right)$,

$$
\liminf _{\lambda \rightarrow \infty}\left\|v_{\lambda}\right\|^{2}=\liminf _{\lambda \rightarrow \infty} \int_{\mathbb{R}^{N}} f\left(t_{\lambda}^{2} v_{\lambda}^{2}\right) v_{\lambda}^{2} \mathrm{~d} x \geq \int_{\mathbb{R}^{N}} \liminf _{\lambda \rightarrow \infty} f\left(t_{\lambda}^{2} v_{\lambda}^{2}\right) v_{\lambda}^{2} \mathrm{~d} x=\infty .
$$

Porém, pelo Teorema da Convergência Dominada de Lebesgue,

$$
\lim _{\lambda \rightarrow \infty} \int_{\mathbb{R}^{N}}\left(\left|\nabla v_{\lambda}\right|^{2}+v_{\lambda}^{2}\right) \mathrm{d} x=\int_{\mathbb{R}^{N}}\left(|\nabla v|^{2}+v^{2}\right) \mathrm{d} x<\infty
$$

Portanto $\left(t_{\lambda}\right)$ é limitada. Analogamente, não existe $\lambda_{n} \rightarrow \infty$ tal que $t_{\lambda_{n}} \rightarrow 0$. Com efeito, se $\lim _{n \rightarrow \infty} t_{\lambda_{n}}=0$, por $\left(f_{5}\right)$,

$$
\left|f\left(t_{\lambda_{n}}^{2} v_{\lambda_{n}}^{2}\right) v_{\lambda_{n}}^{2}\right| \leq \epsilon\left|v_{\lambda_{n}}\right|^{2}+t_{\lambda_{n}}^{q-2} C_{\epsilon}\left|v_{\lambda_{n}}\right|^{q}, \text { para } \epsilon>0 \text { arbitrário, }
$$


e, pelo Teorema da Convergência Dominada de Lebesgue, lembrando que $\left(v_{\lambda_{n}}\right)$ é limitada,

$$
\|v\|^{2}=\lim _{\lambda \rightarrow \infty} \int_{\mathbb{R}^{N}}\left(\left|\nabla v_{\lambda_{n}}\right|^{2}+v_{\lambda_{n}}^{2}\right) \mathrm{d} x=\lim _{n \rightarrow \infty} \int_{\mathbb{R}^{N}}\left|f\left(t_{\lambda_{n}}^{2} v_{\lambda_{n}}^{2}\right) v_{\lambda_{n}}^{2}\right| \mathrm{d} x \leq \epsilon|v|_{2}^{2}, \quad \forall \epsilon>0,
$$

ou seja,

$$
v \equiv 0
$$

o que contradiz $v \in N_{\infty}$. Assim, $\liminf _{\lambda \rightarrow \infty} t_{\lambda}>0$. Seja, então, $\lambda_{n} \rightarrow \infty \operatorname{com} t_{\lambda_{n}} \rightarrow t_{0}$. Daí, como $v \in N_{\infty}$,

$$
\int_{\mathbb{R}^{N}} f\left(v^{2}\right) v^{2} \mathrm{~d} x=\int_{\mathbb{R}^{N}}\left(|\nabla v|^{2}+v^{2}\right) \mathrm{d} x=\int_{\mathbb{R}^{N}} f\left(t_{0}^{2} v^{2}\right) v^{2} \mathrm{~d} x .
$$

Por $\left(f_{4}\right)$, segue que $t_{0}=1$. Como este argumento pode ser usado para qualquer subsequência $\left(\lambda_{n}\right)$, temos $t_{\lambda} \rightarrow 1$ quando $\lambda \rightarrow \infty$.

Pela continuidade do funcional $J_{\infty}$ e do limite acima,

$$
\limsup _{\lambda \rightarrow \infty} c_{\lambda, r} \leq \limsup _{\lambda \rightarrow \infty} J_{\infty}\left(t_{\lambda} v_{\lambda}\right)=J_{\infty}(v)=c_{\infty} .
$$

Por outro lado, pelas definições de $c_{\lambda, r}$ e $c_{\infty}$,

$$
c_{\lambda, r} \geq c_{\infty}
$$

e daí

$$
\liminf _{\lambda \rightarrow \infty} c_{\lambda, r} \geq c_{\infty}
$$

Por (1.14) e (1.15),

$$
\lim _{\lambda \rightarrow \infty} c_{\lambda, r}=c_{\infty}
$$

Seja $R>0$ tal que $\Omega \subset B_{R}(0)$. Para cada $x \in \mathbb{R}^{N}$, denotamos por $\Sigma_{\lambda, x}$ o conjunto

$$
\Sigma_{\lambda, x}:=B_{\lambda R}(x) \backslash \overline{B_{\lambda r}(x)}
$$

e definimos o funcional $\hat{J}_{\lambda, x}: H_{0}^{1}\left(\Sigma_{\lambda, x}, \mathbb{R}\right) \rightarrow \mathbb{R}$ por

$$
\hat{J}_{\lambda, x}(u)=\frac{1}{2} \int_{\Sigma_{\lambda, x}}\left(|\nabla u|^{2}+u^{2}\right) \mathrm{d} x-\frac{1}{2} \int_{\Sigma_{\lambda, x}} F\left(u^{2}\right) \mathrm{d} x
$$

e a variedade Nehari associada a ele

$$
\hat{N}_{\lambda, x}:=\left\{u \in H_{0}^{1}\left(\Sigma_{\lambda, x}, \mathbb{R}\right) \backslash\{0\} ; \hat{J}_{\lambda, x}^{\prime}(u) u=0\right\} .
$$

Para $w \in H^{1}\left(\mathbb{R}^{N}, \mathbb{C}\right)$ com suporte compacto, consideremos a aplicação baricentro

$$
\beta(w):=\frac{\int_{\mathbb{R}^{N}} x|w|^{2} \mathrm{~d} x}{\int_{\mathbb{R}^{N}}|w|^{2} \mathrm{~d} x} .
$$

Definamos o valor

$$
a_{\lambda, x}:=\inf \left\{\hat{J}_{\lambda, x}(v) ; v \in \hat{N}_{\lambda, x}, \beta(v)=x\right\}
$$


Lema 1.12. Temos

$$
c_{\infty}<\liminf _{\lambda \rightarrow \infty} a_{\lambda, 0} .
$$

\section{Demonstração.}

Pela definição de $\hat{N}_{\lambda, 0}$, temos, usando extensão por zero, $\hat{N}_{\lambda, 0} \subset N_{\infty}$ e

$$
c_{\infty} \leq a_{\lambda, 0}, \quad \forall \lambda>0
$$

donde

$$
c_{\infty} \leq \liminf _{\lambda \rightarrow \infty} a_{\lambda, 0} .
$$

Suponhamos, por contradição, que existe $\left(\lambda_{n}\right)$ tal que, quando $n \rightarrow \infty, \lambda_{n} \rightarrow \infty \mathrm{e}$ $a_{\lambda_{n}, 0} \rightarrow c_{\infty}$.

Observe que, para cada $n \in \mathbb{N}$, existe $v_{n} \in H_{0, \text { rad }}^{1}\left(\Sigma_{\lambda_{n}, 0}, \mathbb{R}\right)$ tal que

$$
\hat{J}_{\lambda_{n}, 0}\left(v_{n}\right)=a_{\lambda_{n}, 0} .
$$

Com efeito, argumentando como na Proposição 1.5, $\left.\hat{J}_{\lambda_{n}, 0}\right|_{H_{0, \text { rad }}^{1}\left(\Sigma_{\lambda_{n}, 0}, \mathbb{R}\right)}$ satisfaz $(P S)_{c}$, para todo $c \in \mathbb{R}$. Repetindo a demonstração da Proposição 1.8, $\left.\hat{J}_{\lambda_{n}, 0}\right|_{H_{0, r a d}^{1}\left(\Sigma_{\lambda_{n}, 0}, \mathbb{R}\right) \cap \hat{N}_{\lambda_{n}, 0}}$ também satisfaz $(P S)_{c}$. Assim, pelo Princípio Variacional de Ekeland, para cada $n \in \mathbb{N}$, existe $v_{n} \in H_{0, r a d}^{1}\left(\Sigma_{\lambda_{n}, 0}, \mathbb{R}\right)$ tal que $v_{n} \in \hat{N}_{\lambda_{n}, 0} \subset N_{\infty}$ e

$$
\hat{J}_{\lambda_{n}, 0}\left(v_{n}\right)=a_{\lambda_{n}, 0} \rightarrow c_{\infty} .
$$

Vale ressaltar que $\beta\left(v_{n}\right)=0$, para todo $n \in \mathbb{N}$, e estamos identificando $v_{n} \in H_{0}^{1}\left(\Sigma_{\lambda_{n}, 0}, \mathbb{R}\right)$ com sua extensão por zero no complementar de $\Sigma_{\lambda_{n}, 0}$, a qual é uma função em $H^{1}\left(\mathbb{R}^{N}, \mathbb{R}\right)$. Note que, como $\operatorname{supp} v_{n} \subset \Sigma_{\lambda_{n}, 0}, v_{n}(x) \rightarrow 0$ quase sempre em $\mathbb{R}^{N}$, quando $n \rightarrow \infty$. Além disso, como $\left(v_{n}\right) \subset N_{\infty}$ e $\hat{J}_{\lambda_{n}, 0}\left(v_{n}\right) \rightarrow c_{\infty}$, ela é limitada. Daí $v_{n} \rightarrow 0$ em $H^{1}\left(\mathbb{R}^{N}, \mathbb{R}\right)$. Mais ainda, como $c_{\infty}>0,\left(v_{n}\right)$ não converge fortemente em $H^{1}\left(\mathbb{R}^{N}, \mathbb{R}\right)$. Logo, pela Proposição 1.10 , item $(i i)$,

$$
v_{n}(x)=w_{n}\left(x-y_{n}\right)+\tilde{v}\left(x-y_{n}\right), \quad \forall x \in \mathbb{R}^{N}
$$

$\operatorname{com}\left(w_{n}\right) \subset H^{1}\left(\mathbb{R}^{N}, \mathbb{R}\right)$ satisfazendo $w_{n} \rightarrow 0$ em $H^{1}\left(\mathbb{R}^{N}, \mathbb{R}\right),\left(y_{n}\right) \subset \mathbb{R}^{N}$ tal que $\left|y_{n}\right| \rightarrow \infty$ e $\tilde{v} \in H^{1}\left(\mathbb{R}^{N}, \mathbb{R}\right)$ com

$$
J_{\infty}(\tilde{v})=c_{\infty} \text { e } J_{\infty}^{\prime}(\tilde{v})=0 .
$$

Dada a invariância por rotações do funcional $J_{\infty}$, podemos admitir, sem perder a generalidade, que $y_{n}=\left(y_{n}^{1}, 0, \ldots, 0\right)$, com $y_{n}^{1}<0$.

Lembrando que $\operatorname{supp} v_{n} \subset \Sigma_{\lambda_{n}, 0}$, temos, usando (1.16), o fato de $w_{n} \rightarrow 0$ e o Teorema da Convergência dominada de Lebesgue,

$$
\begin{aligned}
\lim _{n \rightarrow \infty} \int_{\Sigma_{\lambda_{n}, 0} \cap B_{\frac{\lambda_{n} r}{2}}\left(y_{n}\right)}\left|v_{n}\right|^{2} \mathrm{~d} x & =\lim _{n \rightarrow \infty} \int_{B_{\frac{\lambda_{n} r}{2}}\left(y_{n}\right)}\left|v_{n}\right|^{2} \mathrm{~d} x \\
& =\lim _{n \rightarrow \infty} \int_{B_{\lambda_{n} r(0)}}\left|w_{n}-\tilde{v}\right|^{2} \mathrm{~d} x \\
& =\int_{\mathbb{R}^{N}}|\tilde{v}|^{2} \mathrm{~d} x=: M>0 .
\end{aligned}
$$


Mais ainda, como $\mathbb{R}^{N}$ é invariante por translações, assim como a medida de Lebesgue, temos, por um cálculo direto, usando que $w_{n} \rightarrow 0$ em $H^{1}\left(\mathbb{R}^{N}, \mathbb{R}\right)$, quando $n \rightarrow \infty$,

$$
\lim _{n \rightarrow \infty} \int_{\Sigma_{\lambda_{n}, 0}}\left|v_{n}\right|^{2} \mathrm{~d} x=\lim _{n \rightarrow \infty} \int_{\mathbb{R}^{N}}\left|v_{n}\right|^{2} \mathrm{~d} x=\lim _{n \rightarrow \infty} \int_{\mathbb{R}^{N}}\left|w_{n}-\tilde{v}\right|^{2} \mathrm{~d} x=M .
$$

Os dois últimos limites nos dão

$$
\lim _{n \rightarrow \infty} \int_{\Sigma_{\lambda_{n}, 0} \backslash B_{\frac{\lambda_{n} r}{2}}\left(y_{n}\right)}\left|v_{n}\right|^{2} \mathrm{~d} x=0 .
$$

Afirmamos que

$$
\int_{\Sigma_{\lambda_{n}, 0} \cap B_{\frac{\lambda_{n} r}{2}}\left(y_{n}\right)} x^{1}\left|v_{n}\right|^{2} \mathrm{~d} x \leq-\frac{\lambda_{n} r}{2}\left(M+o_{n}(1)\right),
$$

onde $x=\left(x^{1}, x^{2}, \ldots, x^{N}\right) \in \mathbb{R}^{N}$. Com efeito, dado $x \in \Sigma_{\lambda_{n}, 0} \cap B_{\frac{\lambda_{n} r}{2}}\left(y_{n}\right)$, tendo em vista (1.17), basta mostrar que

$$
x^{1}<-\frac{\lambda_{n} r}{2} .
$$

Como $\left|x-y_{n}\right| \leq \frac{\lambda_{n}}{2}$ e $y_{n}=\left(y_{n}^{1}, 0, \ldots, 0\right)$, temos

$$
\left|x^{1}-y_{n}^{1}\right| \leq \frac{\lambda_{n} r}{2}, \quad \sum_{j=2}^{N}\left|x^{j}\right|^{2} \leq\left(\frac{\lambda_{n}}{2}\right)^{2} .
$$

Mais ainda,

$$
\left|x^{1}\right|^{2}+\left(\frac{\lambda_{n}}{2}\right)^{2} \geq\left|x^{1}\right|^{2}+\sum_{j=2}^{N}\left|x^{j}\right|^{2}=\left|x^{2}\right| \geq\left(\lambda_{n} r\right)^{2}
$$

donde

$$
\left|x^{1}\right|^{2} \geq \frac{3}{4}\left(\lambda_{n} r\right)^{2}>\frac{1}{4}\left(\lambda_{n} r\right)^{2}
$$

isto é,

$$
\left|x^{1}\right|>\frac{\lambda_{n} r}{2} .
$$

Portanto, como $y_{n}^{1}<0$ e pela desigualdade em (1.20),

$$
x^{1}<-\frac{\lambda_{n} r}{2} .
$$

como queríamos. Assim, (1.19) é válido.

Por fim, a partir de (1.19) e lembrando que $\operatorname{supp} v_{n} \subset \Sigma_{\lambda_{n}, 0}$,

$$
\begin{aligned}
& 0=\beta\left(v_{n}\right)^{1}=\int_{\Sigma_{\lambda_{n}, 0}} x^{1}\left|v_{n}\right|^{2} \mathrm{~d} x=\int_{\Sigma_{\lambda_{n}, 0} \cap B_{\frac{\lambda_{n} r}{2}}\left(y_{n}\right)} x^{1}\left|v_{n}\right|^{2} \mathrm{~d} x+\int_{\Sigma_{\lambda_{n}, 0 \backslash B_{\frac{\lambda_{n}}{2}}\left(y_{n}\right)}} x^{1}\left|v_{n}\right|^{2} \mathrm{~d} x \\
& \leq-\frac{\lambda_{n} r}{2}\left(M+o_{n}(1)\right)+\lambda_{n} R \int_{\Sigma_{\lambda_{n}, 0} \backslash B_{\frac{\lambda_{n} r}{2}}\left(y_{n}\right)}\left|v_{n}\right|^{2} \mathrm{~d} x
\end{aligned}
$$


e, consequentemente,

$$
\int_{\Sigma_{\lambda_{n}, 0} \backslash B_{\frac{\lambda_{n} r}{2}\left(y_{n}\right)}}\left|v_{n}\right|^{2} \geq \frac{r}{2 R}\left(M+o_{n}(1)\right)>0,
$$

o que contradiz (1.18). Esta contradição mostra que o resultado é válido.

\subsection{Alguns resultados técnicos}

Lembrando que o funcional $J_{\lambda, r}$ restrito à variedade Nehari $N_{\lambda, r}$ satisfaz a condição $(P S)_{c}$, para todo $c \in \mathbb{R}$, concluímos, usando o Princípio Variacional de Ekeland, que existe $u_{\lambda, r} \in N_{\lambda, r} \subset N_{\infty}$ tal que $J_{\lambda, r}\left(u_{\lambda, r}\right)=c_{\lambda, r}$.

Observe que, sem perder a generalidade, podemos supor que $u_{\lambda, r}$ é radial e positiva.

De fato, se $u^{*}$ é a Simetrização de Schwartz de $u_{\lambda, r}$ e $t^{*}>0$ é tal que $t^{*} u^{*} \in N_{\lambda, r}$, então

$$
\int_{B_{\lambda r}(0)}\left|\nabla u^{*}\right|^{2} \mathrm{~d} x \leq \int_{B_{\lambda r}(0)}\left|\nabla u_{\lambda, r}\right|^{2} \mathrm{~d} x, \quad \int_{B_{\lambda r}(0)}\left|u^{*}\right|^{2} \mathrm{~d} x=\int_{B_{\lambda r}(0)}\left|u_{\lambda, r}\right|^{2} \mathrm{~d} x
$$

e, pela monotonicidade de $F$,

$$
\int_{B_{\lambda r}(0)} F\left(\left|u_{\lambda, r}\right|^{2}\right) \mathrm{d} x=\int_{B_{\lambda r}(0)} F\left(\left|u^{*}\right|^{2}\right) \mathrm{d} x
$$

Daí

$$
c_{\lambda, r} \leq J_{\lambda, r}\left(t^{*} u^{*}\right) \leq J_{\lambda, r}\left(t^{*} u_{\lambda, r}\right) \leq \max _{t \geq 0} J_{\lambda, r}\left(t u_{\lambda, r}\right)=J_{\lambda, r}\left(u_{\lambda, r}\right)=c_{\lambda, r}
$$

isto é,

$$
J_{\lambda, r}\left(t^{*} u^{*}\right)=c_{\lambda, r} .
$$

Portanto, $t^{*} u^{*} \in N_{\lambda, r}$ é um ponto crítico de $\left.J_{\lambda, r}\right|_{N_{\lambda, r}}$, donde, pelo Corolário 1.9, é um ponto crítico de $J_{\lambda, r}$. Feito isto, suponhamos, sem perder a generalidade, que $u_{\lambda, r}$ é radial. A positividade segue do fato de, pela teoria de regularidade elíptica, $u_{\lambda, r}$ ser regular e do Princípio do Máximo.

Diminuindo $r>0$ fixado na Seção 1.2, suporemos que os conjuntos

$$
\Omega^{+}:=\left\{x \in \mathbb{R}^{N} ; \operatorname{dist}(x, \Omega) \leq r\right\} \quad \text { e } \quad \Omega^{-}:=\{x \in \Omega ; \operatorname{dist}(x, \partial \Omega) \geq r\}
$$

sejam homotopicamente equivalentes a $\Omega$ (ver Definição A.3).

Definamos a aplicação $\Phi_{\lambda}: \Omega_{\lambda}^{-} \rightarrow M_{\lambda}$ como

$$
\left[\Phi_{\lambda}(y)\right](x):= \begin{cases}t_{\lambda, y} e^{i \tau_{\lambda, y}(x)} u_{\lambda, r}(|x-y|), & x \in B_{\lambda r}(y) \\ 0, & x \notin B_{\lambda r}(y)\end{cases}
$$


onde $t_{\lambda, y}>0$ é tal que $t_{\lambda, y} e^{i \tau_{\lambda, y}(.)} u_{\lambda, r}(|.-y|) \in M_{\lambda}$, e

$$
\tau_{\lambda, y}(x)=\sum_{j=1}^{N} A^{j}\left(\frac{y}{\lambda}\right) x^{j}, \forall x \in \Omega_{\lambda}, \operatorname{com} x=\left(x^{1}, x^{2}, \ldots, x^{N}\right) \in \mathbb{R}^{N} .
$$

Como $u_{\lambda, r}$ é radial, segue que

$$
\beta\left(\Phi_{\lambda}(y)\right)=y, \quad \forall y \in \Omega_{\lambda}^{-} .
$$

Além disso, $\Phi_{\lambda}$ possui a seguinte propriedade:

Lema 1.13. Temos

$$
\lim _{\lambda \rightarrow \infty} \max _{y \in \Omega_{\lambda}^{-}}\left|I_{\lambda}\left(\Phi_{\lambda}(y)\right)-c_{\infty}\right|=0
$$

\section{Demonstração.}

Usaremos o seguinte resultado: sejam, para cada $n \in \mathbb{N}, f_{n}: \Omega_{n} \rightarrow \mathbb{R}$ funções contínuas, $\Omega_{n} \subset \mathbb{R}^{N}$ conjuntos compactos e $a \in \mathbb{R}$. São equivalentes:

- $\lim _{n \rightarrow \infty} f_{n}\left(y_{n}\right)=a$, para qualquer sequência $\left(y_{n}\right) \subset \mathbb{R}^{N}$ tal que $y_{n} \in \Omega_{n}$;

- $\lim _{n \rightarrow \infty} \max _{y \in \Omega_{n}}\left|f_{n}(y)-a\right|=0$.

Dessa forma, mostremos que, dadas $\left(\lambda_{n}\right) \subset \mathbb{R} \operatorname{com} \lambda_{n} \rightarrow \infty$ e $\left(y_{n}\right) \subset \Omega_{\lambda_{n}}^{-}$quaisquer,

$$
I_{\lambda_{n}}\left(\Phi_{\lambda_{n}}\left(y_{n}\right)\right) \rightarrow c_{\infty} \text {, quando } n \rightarrow \infty
$$

Como $J_{\lambda_{n}, r}\left(u_{\lambda_{n}, r}\right)=c_{\lambda_{n}, r}, u_{\lambda_{n}, r} \in N_{\lambda_{n}, r} \subset N_{\infty}$, temos, fazendo $u_{n}:=u_{\lambda_{n}, r}$

$$
\lim _{n \rightarrow \infty} J_{\infty}\left(u_{n}\right)=c_{\infty}, \quad J_{\infty}^{\prime}\left(u_{n}\right) u_{n}=0 \quad \text { e } \quad J_{\infty}\left(t u_{n}\right) \leq J_{\infty}\left(u_{n}\right)=c_{\lambda_{n}, r}
$$

Argumentando como na prova do Lema 1.11, existe $u \not \equiv 0$ tal que $u_{n} \rightarrow u$ em $H^{1}\left(\mathbb{R}^{N}, \mathbb{R}\right)$ quando $n \rightarrow \infty$. A primeira igualdade acima implica, pela Proposição $1.10(i i)$,

$$
u_{n} \rightarrow u \text { em } H^{1}\left(\mathbb{R}^{N}, \mathbb{R}\right) \text {, quando } n \rightarrow \infty \text {. }
$$

Além disso, como $H_{r a d}^{1}\left(\mathbb{R}^{N}, \mathbb{R}\right) \hookrightarrow L^{2}\left(\mathbb{R}^{N}, \mathbb{R}\right)$ compactamente,

$$
u_{n} \rightarrow u \text { em } L^{2}\left(\mathbb{R}^{N}, \mathbb{R}\right) \text { e } u_{n}(x) \rightarrow u(x) \text { quase sempre em } \mathbb{R}^{N} \text {, quando } n \rightarrow \infty \text {. }
$$

Seja $t_{n}:=t_{\lambda_{n}, y_{n}}$ como na definição de $\Phi_{\lambda_{n}}$.

Notemos que, pela definição de $\nabla_{A_{\lambda}}$,

$$
\left|\nabla_{A_{\lambda_{n}}} \Phi_{\lambda_{n}}\left(y_{n}\right)\right|^{2}=\sum_{j=1}^{N}\left|D_{A_{\lambda_{n}}}^{j}\left(\Phi_{\lambda_{n}}\left(y_{n}\right)\right)\right|^{2},
$$


com, para $x \in \Omega_{\lambda_{n}}$

$$
\begin{aligned}
D_{A_{\lambda_{n}}}^{j}\left(\Phi_{\lambda_{n}}\left(y_{n}\right)(x)\right)= & -i \partial_{j} \Phi_{\lambda_{n}}\left(y_{n}\right)(x)-A^{j}\left(\frac{x}{\lambda_{n}}\right) \Phi_{\lambda_{n}}\left(y_{n}\right)(x) \\
= & t_{n} e^{i \tau_{\lambda_{n}, y_{n}}(x)}\left[-A^{j}\left(\frac{x}{\lambda_{n}}\right) u_{n}\left(\left|x-y_{n}\right|\right)-i \partial_{j}\left(u_{n}\left(\left|x-y_{n}\right|\right)+\right.\right. \\
& \left.+u_{n}\left(\left|x-y_{n}\right|\right) A^{j}\left(\frac{y_{n}}{\lambda_{n}}\right)\right] \\
= & t_{n} e^{i \tau_{\lambda_{n}, y_{n}}(x)}\left[u_{n}\left(\left|x-y_{n}\right|\right)\left(A^{j}\left(\frac{y_{n}}{\lambda_{n}}\right)-A^{j}\left(\frac{x}{\lambda_{n}}\right)\right)-\right. \\
& \left.-i \partial_{j} u_{n}\left(\left|x-y_{n}\right|\right)\right]
\end{aligned}
$$

Portanto

$$
\begin{aligned}
\left|\nabla_{A_{\lambda_{n}}} \Phi_{\lambda_{n}}\left(y_{n}\right)\right|^{2}= & t_{n}^{2}\left[u_{n}^{2}\left(\left|x-y_{n}\right|\right)\left|A\left(\frac{y_{n}}{\lambda_{n}}\right)-A\left(\frac{x}{\lambda_{n}}\right)\right|^{2}+\right. \\
& \left.+\left|\nabla u_{n}\left(\left|x-y_{n}\right|\right)\right|^{2}\right]
\end{aligned}
$$

e pela forma como $t_{n}$ e $u_{n}$ foram escolhidos,

$$
\begin{aligned}
I_{\lambda_{n}}\left(\Phi_{\lambda_{n}}\left(y_{n}\right)\right) & =J_{\lambda_{n}, r}\left(t_{n} u_{n}\right)+\frac{t_{n}^{2}}{2} \int_{B_{\lambda_{n} r}(0)}\left|A\left(\frac{y_{n}}{\lambda_{n}}\right)-A\left(\frac{y_{n}+z}{\lambda_{n}}\right)\right|^{2}\left|u_{n}(z)\right|^{2} \mathrm{~d} z \\
& =J_{\lambda_{n}, r}\left(u_{n}\right)+\frac{t_{n}^{2}}{2} \int_{B_{\lambda_{n} r}(0)}\left|A\left(\frac{y_{n}}{\lambda_{n}}\right)-A\left(\frac{y_{n}+z}{\lambda_{n}}\right)\right|^{2}\left|u_{n}(z)\right|^{2} \mathrm{~d} z \\
& =c_{\lambda_{n}, r}+\frac{t_{n}^{2}}{2} \int_{B_{\lambda_{n} r}(0)}\left|A\left(\frac{y_{n}}{\lambda_{n}}\right)-A\left(\frac{y_{n}+z}{\lambda_{n}}\right)\right|^{2}\left|u_{n}(z)\right|^{2} \mathrm{~d} z
\end{aligned}
$$

Além disso,

$$
\begin{aligned}
I_{\lambda_{n}}\left(\Phi_{\lambda_{n}}\left(y_{n}\right)\right) & \geq I_{\lambda_{n}}\left(e^{i \tau_{\lambda_{n}, y_{n}}} u_{n}\right) \\
& =J_{\lambda_{n}, r}\left(u_{n}\right)+\frac{1}{2} \int_{B_{\lambda_{n} r}(0)}\left|A\left(\frac{y_{n}}{\lambda_{n}}\right)-A\left(\frac{y_{n}+z}{\lambda_{n}}\right)\right|^{2}\left|u_{n}(z)\right|^{2} \mathrm{~d} z \\
& \geq c_{\lambda_{n}, r} .
\end{aligned}
$$

Logo

$$
c_{\lambda_{n}, r} \leq I_{\lambda_{n}}\left(\Phi_{\lambda_{n}}\left(y_{n}\right)\right)=c_{\lambda_{n}, r}+\frac{t_{n}^{2}}{2} \int_{B_{\lambda_{n} r}(0)}\left|A\left(\frac{y_{n}}{\lambda_{n}}\right)-A\left(\frac{y_{n}+z}{\lambda_{n}}\right)\right|^{2}\left|u_{n}(z)\right|^{2} \mathrm{~d} z .
$$

Como $\frac{y_{n}}{\lambda_{n}} \in \Omega$ e $\Omega$ é limitado, existe $y_{0} \in \bar{\Omega}$ tal que $\frac{y_{n}}{\lambda_{n}} \rightarrow y_{0}$ quando $n \rightarrow \infty$. Como na demonstração do Lema 1.10,

$$
\frac{t_{n}^{2}}{2} \int_{B_{\lambda_{n} r}(0)}\left|A\left(\frac{y_{n}}{\lambda_{n}}\right)-A\left(\frac{y_{n}+z}{\lambda_{n}}\right)\right|^{2}\left|u_{n}(z)\right|^{2} \mathrm{~d} z=o_{n}(1) .
$$


Assim, novamente pelo Lema 1.10 e (1.22),

$$
\lim _{n \rightarrow \infty} I_{\lambda_{n}}\left(\Phi_{\lambda_{n}}\left(y_{n}\right)\right)=c_{\infty}
$$

como queríamos.

Consideremos

$$
g(\lambda):=\sup _{y \in \Omega_{\lambda}^{-}} I_{\lambda}\left(\Phi_{\lambda}(y)\right)
$$

Então o conjunto

$$
O_{\lambda}:=\left\{u \in M_{\lambda} ; I_{\lambda}(u) \leq g(\lambda)\right\}
$$

é não vazio e, pelo Lema 1.13,

$$
\Phi_{\lambda}\left(\Omega_{\lambda}^{-}\right) \subset O_{\lambda} .
$$

Note que, dado $u \in M_{\lambda}$, existe $t_{u}>0$ tal que $t_{u}|u| \in N_{\lambda}$. pela desigualdade diamagnética, se $u \in M_{\lambda}$,

$$
\||u|\|^{2} \leq\|u\|_{A_{\lambda}}^{2}=\int_{\Omega_{\lambda}} f\left(|u|^{2}\right)|u|^{2} \mathrm{~d} x .
$$

Vejamos que $t_{u} \in[0,1]$. Definamos $h_{u}:(0, \infty) \rightarrow \mathbb{R}$ por $h_{u}(t):=J_{\lambda}(t|u|)$. Como $t_{u}|u| \in N_{\lambda}$,

$$
\int_{\Omega_{\lambda}}\left(|\nabla| u||^{2}+|u|^{2}\right) \mathrm{d} x-\int_{\Omega_{\lambda}} f\left(t_{u}^{2}|u|^{2}\right)|u|^{2} \mathrm{~d} x=\frac{1}{t_{u}} J_{\lambda}^{\prime}\left(t_{u}|u|\right)|u|=0
$$

donde, por (1.25),

$$
\int_{\Omega_{\lambda}} f\left(t_{u}^{2}|u|^{2}\right)|u|^{2} \mathrm{~d} x \leq \int_{\Omega_{\lambda}} f\left(|u|^{2}\right)|u|^{2} \mathrm{~d} x
$$

e, por $\left(f_{4}\right), t_{u} \in[0,1]$.

Proposição 1.14. Existe $\hat{\lambda}>0$ tal que $\beta(u) \in \Omega_{\lambda}^{+}$, se $u \in O_{\lambda}$ e $\lambda \geq \hat{\lambda}$.

\section{Demonstração.}

Suponhamos, por contradição, que o resultado seja falso. Então existem $\lambda_{n} \stackrel{n \rightarrow \infty}{\rightarrow} \infty \mathrm{e}$ $u_{n} \in M_{\lambda_{n}}$ tais que

$$
I_{\lambda_{n}}\left(u_{n}\right) \geq g\left(\lambda_{n}\right), \operatorname{com} x_{n}:=\beta\left(u_{n}\right) \notin \Omega_{\lambda_{n}}^{+} .
$$

Seja $t_{n} \in[0,1]$ tal que $v_{n}:=t_{n}\left|u_{n}\right| \in N_{\lambda_{n}}$. Segue, da desigualdade diamagnética e de $u_{n} \in O_{\lambda_{n}}$,

$$
J_{\lambda_{n}}\left(v_{n}\right) \leq I_{\lambda_{n}}\left(t_{n} u_{n}\right) \leq I_{\lambda_{n}}\left(u_{n}\right) \leq g\left(\lambda_{n}\right) .
$$

Assim, a sequência $\left(v_{n}\right)$ satisfaz

$$
v_{n} \in N_{\lambda_{n}}, \beta\left(v_{n}\right)=x_{n} \notin \Omega_{\lambda_{n}}^{+}, J_{\lambda_{n}}\left(v_{n}\right) \leq g\left(\lambda_{n}\right) .
$$

Observe que

$$
\Omega_{\lambda_{n}} \subset \Sigma_{\lambda_{n}, x_{n}}
$$


Com efeito, seja $x \in \Omega_{\lambda_{n}}$. Então $\lambda_{n}{ }^{-1} x \in \Omega$ e $\lambda_{n} x_{n} \notin \Omega^{+}$. Da definição de $\Omega^{+}$,

$$
\left|x-x_{n}\right| \geq \lambda_{n} r
$$

Por outro lado, como $x=\lambda_{n} y_{n}$, para algum $y \in \Omega$, temos, lembrando a forma como $R>0$ foi tomado,

$$
\begin{aligned}
\left|x-x_{n}\right| & =\left|x-\frac{\int_{\lambda_{n}} z\left|v_{n}\right|^{2} \mathrm{~d} z}{\int_{\Omega_{\lambda_{n}}}\left|v_{n}\right|^{2} \mathrm{~d} z}\right|=\left|\frac{\int_{\Omega_{\lambda_{n}}}\left(\lambda_{n} y-z\right)\left|v_{n}\right|^{2} \mathrm{~d} z}{\int_{\Omega_{\lambda_{n}}}\left|v_{n}\right|^{2} \mathrm{~d} z}\right| \\
& =\lambda_{n}\left|\frac{\iint_{\lambda_{n}}\left(y-\frac{z}{\lambda_{n}}\right)\left|v_{n}\right|^{2} \mathrm{~d} z}{\int_{\Omega_{\lambda_{n}}}\left|v_{n}\right|^{2} \mathrm{~d} z}\right| \\
& \leq \lambda_{n}\left|y-\frac{z}{\lambda_{n}}\right| \leq \lambda_{n} \operatorname{diam}(\Omega)<\lambda_{n} R .
\end{aligned}
$$

Isto e (1.27) implicam que

$$
x \in B_{\lambda_{n} R}\left(x_{n}\right) \backslash \overline{B_{\lambda_{n} r}\left(x_{n}\right)}=\Sigma_{\lambda_{n}, x_{n}}
$$

e como $x \in \Omega_{\lambda_{n}}$ é qualquer, vale (1.26).

Por fim, usando (1.26), temos $v_{n} \in H_{0}^{1}\left(\Sigma_{\lambda_{n}, x_{n}}, \mathbb{R}\right)$. como $x_{n}=\beta\left(v_{n}\right)$ e usando o fato de $H_{0}^{1}\left(\Sigma_{\lambda_{n}, 0}, \mathbb{R}\right)$ e $H_{0}^{1}\left(\Sigma_{\lambda_{n}, x_{n}}, \mathbb{R}\right)$ serem isometricamente isomorfos, donde $a_{\lambda_{n}, 0}=a_{\lambda_{n}, x_{n}}$,

$$
a_{\lambda_{n}, 0}=a_{\lambda_{n}, x_{n}} \leq \hat{J}_{\lambda_{n}, x_{n}}\left(v_{n}\right)=J_{\lambda_{n}}\left(v_{n}\right) \leq g\left(\lambda_{n}\right) .
$$

A definição de $g$ e o Lema 1.13 nos dão

$$
g\left(\lambda_{n}\right) \rightarrow c_{\infty}, \text { quando } n \rightarrow \infty
$$

Logo

$$
\limsup _{n \rightarrow \infty} a_{\lambda_{n}, 0} \leq \limsup _{n \rightarrow \infty} g\left(\lambda_{n}\right)=c_{\infty}
$$

o que contradiz o Lema 1.12. Portanto o resultado é válido.

\subsection{Prova do Teorema 1.1}

Proposição 1.15. Seja $\hat{\lambda}>0$ dado na Proposição 1.14. Para cada $\lambda \geq \hat{\lambda}$,

$$
\operatorname{cat}\left(O_{\lambda}\right) \geq \operatorname{cat}(\Omega)
$$

\section{Demonstração.}


Suponhamos que $\operatorname{cat}\left(O_{\lambda}\right)=n$. Então existem $\Upsilon_{j} \subset O_{\lambda}, j=1, \ldots, n$, fechados e contráteis em $O_{\lambda}$, tais que

$$
O_{\lambda}=\bigcup_{j=1}^{n} \Upsilon_{j}
$$

e $h_{j} \in C\left([0,1] \times \Upsilon_{j}, O_{\lambda}\right)$ tais que

$$
h_{j}(0, u)=u, h_{j}(1, u)=u_{j} \in O_{\lambda}, \text { para cada } u \in \Upsilon_{j}, j=1, \ldots, n,
$$

e $u_{j} \in O_{\lambda}$ fixos.

Considere os conjuntos $B_{j}:=\Phi^{-1}\left(\Upsilon_{j}\right), j=1, \ldots, n$, os quais são fechados em $\Omega_{\lambda}^{-}$e

$$
\Omega_{\lambda}^{-}=\bigcup_{j=1}^{n} B_{j} .
$$

Pela Proposição 1.14 , as aplicações $g_{j}:[0,1] \times B_{j} \rightarrow \Omega_{\lambda}^{+}$dadas por

$$
g_{j}(t, y):=\beta\left(h_{j}\left(t, \Phi_{\lambda}(y)\right)\right)
$$

estão bem definidas. Mais ainda, $g_{j} \in C\left([0,1] \times B_{j}, \Omega_{\lambda}^{+}\right)$e

$$
g_{j}(0, y)=y, g_{j}(1, y)=y_{j} \in \Omega_{\lambda}^{+}, \text {para cada } y \in B_{j}, j=1, \ldots, n,
$$

e $y_{j} \in \Omega_{\lambda}^{+}$fixos. Portanto, como $\Omega_{\lambda}^{+}$e $\Omega_{\lambda}^{-}$são homotopicamente equivalentes a $\Omega_{\lambda}$,

$$
\operatorname{cat}\left(\Omega_{\lambda}\right)=\operatorname{cat}_{\Omega^{+}} \Omega_{\lambda}^{-} \leq n,
$$

como queríamos.

\section{Demonstração do Teorema 1.1.}

Tomemos $g(\lambda)>0$ dado em (1.23), $\hat{\lambda}>0$ dado na Proposição 1.14 e $\lambda>\lambda^{*}$. Notemos que $g(\lambda)$ não é um valor crítico de $I_{\lambda}$. De fato, primeiramente observemos que $g(\lambda)=g(\lambda, r)$, $r>0$ fixado de modo que $\Omega_{\lambda}^{+}$e $\Omega_{\lambda}^{-}$são homotopicamente equivalentes a $\Omega_{\lambda}$. Podemos repetir os argumentos feitos até aqui para $\rho \in(0, r)$ no lugar de $r$. Se $g(\lambda)$ é uma valor crítico para todo $\rho \in(0, r]$, então $I_{\lambda}$ possui infinitos pontos críticos e a prova está completa. Suponhamos, então, sem perder a generalidade, que $g(\lambda)$ é um valor regular, diminuindo $r>0$ se necessário. Como $M_{\lambda}^{g(\lambda)}$ é um conjunto fechado de $M_{\lambda}$, tendo em vista as Proposição $1.8, I_{\lambda}$ restrito a $M_{\lambda}^{g(\lambda)}$ satisfaz $(P S)_{d}$, para todo $d \in \mathbb{R}$. Logo podemos aplicar a teoria de Ljusternik-Schnirelmann e a Proposição 1.15 para obtemos cat $\left(\Omega_{\lambda}\right)$ pontos críticos de $\left.I_{\lambda}\right|_{M_{\lambda}^{g(\lambda)}}$. Pelo fato de $g(\lambda)$ ser valor regular, obtemos $\operatorname{cat}\left(\Omega_{\lambda}\right)$ pontos críticos de $\left.I_{\lambda}\right|_{M_{\lambda}}$. Pelo Corolário 1.9, estes são pontos críticos de $I_{\lambda}$. 


\subsection{Provas dos Teorema 1.2 e Corolário 1.3}

Nesta seção usaremos os resultados mostrados na Seção A.3 do Apêndice A. Verifiquemos, então, que as condições exigidas na referida seção são satisfeitas. De fato:

- a condição $(i)$ segue da forma como o funcional $I_{\lambda}$ foi definido e das hipóteses sobre $f$;

- (ii) segue da Proposição 1.5 e de $H_{I_{\lambda}}(u): E_{A_{\lambda}} \times E_{A_{\lambda}} \rightarrow \mathbb{R}$ dada por $H_{I_{\lambda}}(u)(v, w)=\langle v, w\rangle_{A_{\lambda}}-\int_{\Omega_{\lambda}}\left(2 f^{\prime}\left(|u|^{2}\right) \operatorname{Re}(u \bar{v}) \operatorname{Re}(u \bar{w})+f\left(|u|^{2}\right) \operatorname{Re}(v \bar{w})\right) \mathrm{d} x, \forall v, w \in E_{A_{\lambda}}$, ser uma forma bilinear simétrica para todo $u \in E_{A_{\lambda}}$;

- $(i i i)$ e $(i v)$ são consequências da Proposição 1.7 - vale ressaltar que $\delta_{0}>0$ independente de $\lambda>0$;

- $(v)$ segue por (1.24) e pela Proposição 1.14 para $\lambda>\hat{\lambda}$ : temos $\Phi_{\lambda}: \Omega_{\lambda}^{-} \rightarrow O_{\lambda}$ e $\beta: \Omega_{\lambda}^{+} \rightarrow O_{\lambda}$, onde $O_{\lambda}=M_{\lambda}^{g(\lambda)}, g(\lambda)$ valor regular de $I_{\lambda}$ definido em (1.23), e, por construção $\beta \circ \Phi_{\lambda}=I d_{\Omega_{\lambda}^{-}}, \Omega_{\lambda}^{+}, \Omega_{\lambda}^{-}$e $\Omega$ homotopicamente equivalentes.

Assim, tomando, para $\lambda>\hat{\lambda}$, os entes matemáticos deste capítulo que correspondem aos que foram fixados na Seção A.3 do Apêndice A, vamos às demonstrações dos resultados principais.

\section{Demonstração do Teorema 1.2.}

Seja $\lambda \geq \hat{\lambda}, \hat{\lambda}>0$ dado na Proposição 1.14. Seja também $\mathcal{K}$ o conjunto dos pontos críticos de $I_{\lambda}$. Suponha que $\mathcal{K}$ é discreto.

Definamos

$$
\mathcal{C}_{1}:=\left\{u \in \mathcal{K} ; \delta<I_{\lambda}(u) \leq g(\lambda)\right\}
$$

e

$$
\mathcal{C}_{2}:=\left\{u \in \mathcal{K} ; g(\lambda)<I_{\lambda}(u)\right\}
$$

$\operatorname{com} \delta \in\left(0, \delta_{0}\right)$ dado na Proposição $1.7, g(\lambda)$ como em (1.23). Então, como $\mathcal{C}_{1}, \mathcal{C}_{2}$ são conjuntos críticos isolados e $\mathcal{K}=\mathcal{C}_{1} \cup \mathcal{C}_{2}$, pelas propriedades do índice de Morse generalizado

$$
i_{t}\left(\mathcal{K}, I_{\lambda}\right)=i_{t}\left(\mathcal{C}_{1}, I_{\lambda} \mid \frac{}{\Sigma(\delta, g(\lambda))}\right)+i_{t}\left(\mathcal{C}_{2}, I_{\lambda} \mid \overline{\Sigma(g(\lambda),+\infty)}\right)=\sum_{u \in \mathcal{C}_{1}} i_{t}(u)+\sum_{u \in \mathcal{C}_{2}} i_{t}(u) .
$$

Pelo Lema A.26,

$$
\sum_{u \in \mathcal{C}_{1}} i_{t}(u)=t \mathcal{P}_{t}\left(\Omega_{\lambda}\right)+t \mathcal{Q}(t)+(1+t) \mathcal{Q}_{1}(t)
$$

$\mathrm{e}$

$$
\sum_{u \in \mathcal{C}_{2}} i_{t}(u)=t^{2}\left[\mathcal{P}_{t}\left(\Omega_{\lambda}\right)+\mathcal{Q}(t)-1\right]+(1+t) \mathcal{Q}_{2}(t)
$$


Então, como $\mathcal{Q}$ é um polinômio com coeficientes não negativos,

$$
\sum_{u \in \mathcal{K}} i_{t}(u)=t \mathcal{P}_{t}\left(\Omega_{\lambda}\right)+t^{2}\left[\mathcal{P}_{t}\left(\Omega_{\lambda}\right)-1\right]+(1+t) \mathcal{Q}_{3}(t),
$$

onde $\mathcal{Q}_{3}$ é um polinômio com coeficientes não negativos.

\section{Demonstração do Corolário 1.3.}

Seja $\lambda \geq \hat{\lambda}, \hat{\lambda}>0$ dado na Proposição 1.14. Seja também $\mathcal{K}$ o conjunto dos pontos críticos de $I_{\lambda}$. Suponha que todos os pontos críticos de $I_{\lambda}$ são não degenerados. Basta, então, observar que, nestas circunstâncias,

$$
i(u)=t^{m(u)} \text {, para todo } u \in \mathcal{K} .
$$





\section{Múltiplas soluções para uma equação de Schrödinger com campo magnético}

Estamos interessados em aplicar a teoria de Morse ao problema

$$
\left\{\begin{aligned}
(-i \nabla-A)^{2} u+\kappa u & =|u|^{p-2} u, \quad \text { em } \Omega \\
u & =0 \text { sobre } \partial \Omega
\end{aligned}\right.
$$

onde $\kappa$ é um parâmetro real positivo, $\Omega \subset \mathbb{R}^{N}$ é um domínio limitado, $N \geq 3$, $i$ é a unidade imaginária, $A: \Omega \rightarrow \mathbb{R}$ uma aplicação contínua e $p \in\left(2,2^{*}\right)$.

Motivados pelos trabalhos de Benci and Cerami [10], nosso objetivo é mostrar os seguintes resultados:

Teorema 2.1. Existe uma função $\bar{p}:[0,+\infty) \rightarrow\left(2,2^{*}\right)$ tal que para todo $p \in\left[\bar{p}(\kappa), 2^{*}\right)$, o problema $\left(P_{A, \kappa, p}\right)$ possui, pelo menos, cat $(\Omega)$ soluções não triviais.

Teorema 2.2. Seja $\bar{p}:[0,+\infty) \rightarrow\left(2,2^{*}\right)$ a função dada no Teorema 2.1. Suponha que o conjunto $\mathcal{K}$ de soluções do problema $\left(P_{A, \kappa, p}\right)$ é discreto. Então, para todo $p \in\left[\bar{p}(\kappa), 2^{*}\right)$,

$$
\sum_{u \in \mathcal{K}} i_{t}(u)=t \mathcal{P}_{t}(\Omega)+t^{2}\left[\mathcal{P}_{t}(\Omega)-1\right]+(t+1) \mathcal{Q}(t)
$$


34Capítulo 2 - Multiplicidade de soluções via teoria de Morse: problema de Dirichlet homogêneo

onde $\mathcal{Q}$ é um polinômio com coeficientes inteiros não negativos, $\mathcal{P}_{t}(\Omega)$ é o polinômio de Poincaré de $\Omega$ e $i_{t}(u)$ é o índice de Morse generalizado de u.

No caso não degenerado, temos

Corolário 2.3. Seja $\bar{p}:[0,+\infty) \rightarrow\left(2,2^{*}\right)$ a função dada no Teorema 2.1. Suponha que as soluções de $\left(P_{A, \kappa, p}\right)$ sejam não degeneradas. Então, para todo $p \in\left[\bar{p}(\kappa), 2^{*}\right)$,

$$
\sum_{u \in \mathcal{K}} t^{m(u)}=t \mathcal{P}_{t}(\Omega)+t^{2}\left[\mathcal{P}_{t}(\Omega)-1\right]+(t+1) \mathcal{Q}(t),
$$

onde $\mathcal{Q}$ é um polinômio com coeficientes inteiros não negativos, $\mathcal{P}_{t}(\Omega)$ é o polinômio de Poincaré de $\Omega$ e $m_{t}(u)$ é o índice de Morse de u.

A partir do Corolário 2.3 , concluímos que $\left(P_{A, \kappa, p}\right)$ possui, pelo menos, $2 \mathcal{P}_{1}(\Omega)-1$ soluções não triviais.

\subsection{Condição (PS)}

Denotemos por $E_{A}$ o espaço de Hilbert sobre o corpo dos reais obtido como o fechamento de $C_{c}^{\infty}(\Omega, \mathbb{C})$ com a norma induzida pelo produto escalar

$$
\langle u, v\rangle_{A}:=\operatorname{Re}\left\{\int_{\Omega} \nabla_{A} u \overline{\nabla_{A} v} \mathrm{~d} x\right\},
$$

onde $\operatorname{Re}(w)$ é a parte real de $w \in \mathbb{C}$ e $\bar{w}$ é o seu conjugado complexo,

$$
\nabla_{A} u:=\left(D_{A}^{j} u\right)_{j=1}^{N}, \quad D_{A}^{j} u=-i \partial_{j} u-A^{j} u, \quad j \in 1, \ldots, N .
$$

A norma induzida pelo produto interno acima é

$$
\|u\|_{A}^{2}:=\int_{\Omega}\left|\nabla_{A} u\right|^{2} \mathrm{~d} x .
$$

Temos, para $u \in E_{A}$, a desigualdade diamagnética (ver Lema 1.4), provada por Esteban e Lions [26].

Diremos que $u \in E_{A}$ é uma solução fraca do problema $\left(P_{A, \kappa, p}\right)$ se

$$
\operatorname{Re}\left(\int_{\Omega}\left(\nabla_{A} u \overline{\nabla_{A} v}+\kappa u \bar{v}-|u|^{p-2} u \bar{v}\right) \mathrm{d} x\right)=0, \forall v \in E_{A} .
$$

Seja o funcional $I_{\kappa, p, \Omega}: E_{A} \rightarrow \mathbb{R}$ dado por

$$
I_{\kappa, p, \Omega}(u)=\frac{1}{2} \int_{\Omega}\left(\left|\nabla_{A} u\right|^{2}+\kappa|u|^{2}\right) \mathrm{d} x-\frac{1}{p} \int_{\Omega}|u|^{p} \mathrm{~d} x, \forall u \in E_{A},
$$

o qual, pelas imersões de Sobolev e a Desigualdade Diamagnética, está bem definido. Mais ainda, $I_{\kappa, p, \Omega} \in C^{2}\left(E_{A}, \mathbb{R}\right)$ com

$$
I_{\kappa, p, \Omega}^{\prime}(u) v=\operatorname{Re}\left(\int_{\Omega}\left(\nabla_{A} u \overline{\nabla_{A} v}+\kappa u \bar{v}-|u|^{p-2} u \bar{v}\right) \mathrm{d} x\right), \forall u, v \in E_{A} .
$$

Assim, as soluções fracas de $\left(P_{A, \kappa, p}\right)$ são os pontos críticos de $I_{\kappa, p, \Omega}$. 
Proposição 2.4. $I_{\kappa, p, \Omega}$ satisfaz $(P S)_{c}$, para todo $c \in \mathbb{R}$.

\section{Demonstração.}

Segue os mesmos passos da demonstração da Proposição 1.5.

Argumentando como na Proposição 1.6, o funcional $I_{\kappa, p, \Omega}$ possui a geometria do passo da montanha. Dessa forma, pela Proposição 2.4 e pelo Teorema do Passo da Montanha de Ambrosetti- Rabinowitz, para cada $\kappa>0, p \in\left(2,2^{*}\right),\left(P_{A, \kappa, p}\right)$ possui pelo menos uma solução não-trivial $u \in E_{A}$ tal que $I_{\kappa, p, \Omega}(u)=b_{\kappa, p, \Omega}$ e $I_{\kappa, p, \Omega}^{\prime}(u)=0$, onde $b_{\kappa, p, \Omega}$ denota o nível do passo da montanha de $I_{\kappa, p, \Omega}$. Argumentando como em [43, Theorem 4.2], temos

$$
b_{\kappa, p, \Omega}:=\inf _{u \in M_{\kappa, p, \Omega}} I_{\kappa, p, \Omega}(u),
$$

com

$$
M_{\kappa, p, \Omega}=\left\{u \in E_{A} \backslash\{0\} ; I_{\kappa, p, \Omega}^{\prime}(u) u=0\right\},
$$

a variedade Nehari de $I_{\kappa, p, \Omega}$.

Sobre o funcional $I_{\kappa, p, \Omega}$ e a variedade $M_{\kappa, p, \Omega}$ temos os seguintes resultados, cujas demonstrações são idênticas às das Proposições 1.7 e 1.8 .

Proposição 2.5. A variedade $M_{\kappa, p, \Omega}$ é difeomorfa à esfera unitária em $E_{A}$. Além disso, existe $\delta_{p}>0$ independente de $\kappa>0$ tal que, para todo $u \in M_{\kappa, p, \Omega}$,

$$
\int_{\Omega}\left(\left|\nabla_{A} u\right|^{2}+\kappa|u|^{2}\right) \mathrm{d} x \geq \delta_{p} \quad e \quad I_{\kappa, p, \Omega}(u) \geq \delta_{p} .
$$

Proposição 2.6. O funcional $\left.I_{\kappa, p, \Omega}\right|_{M_{\kappa, p, \Omega}}$ satisfaz a condição $(P S)_{d}$, para todo $d \in \mathbb{R}$. Como consequência das proposições anteriores, temos

Corolário 2.7. Todo ponto crítico de $\left.I_{\kappa, p, \Omega}\right|_{M_{\kappa, p, \Omega}}$ é um ponto crítico de $I_{\kappa, p, \Omega}$.

\subsection{Comportamento dos níveis minimax}

Definamos

$$
m_{A}(\kappa, p, \Omega):=\inf _{u \in E_{A} \backslash\{0\}} \frac{\int_{\Omega}\left(\left|\nabla_{A} u\right|^{2}+\kappa|u|^{2}\right) \mathrm{d} x}{\left(\int_{\Omega}|u|^{p} \mathrm{~d} x\right)^{\frac{2}{p}}}
$$

e ponhamos

$$
S_{A, \kappa}:=m_{A}\left(\kappa, 2^{*}, \Omega\right), \quad S_{A}:=m_{A}\left(0,2^{*}, \Omega\right) .
$$

Lema 2.8. Seja $b_{\kappa, p, \Omega}$ o nivel do passo da montanha do funcional $I_{\kappa, p, \Omega}$. Então

$$
b_{\kappa, p, \Omega}=\left(\frac{1}{2}-\frac{1}{p}\right) m_{A}(\kappa, p, \Omega)^{\frac{p}{p-2}} .
$$




\section{Demonstração.}

Com efeito, seja $u \in M_{\kappa, p, \Omega}$. Então

$$
\int_{\Omega}\left(\left|\nabla_{A} u\right|^{2}+\kappa|u|^{2}\right) \mathrm{d} x=\int_{\Omega}|u|^{p} \mathrm{~d} x .
$$

Logo

$$
b_{\kappa, p, \Omega} \leq I_{\kappa, p, \Omega}(u)=\left(\frac{1}{2}-\frac{1}{p}\right) \int_{\Omega}\left(\left|\nabla_{A} u\right|^{2}+\kappa|u|^{2}\right) \mathrm{d} x
$$

donde

$$
\int_{\Omega}\left(\left|\nabla_{A} u\right|^{2}+\kappa|u|^{2}\right) \mathrm{d} x \geq\left(\frac{1}{2}-\frac{1}{p}\right)^{-1} b_{\kappa, p, \Omega}
$$

Além disso,

$$
m_{A}(\kappa, p, \Omega) \leq \frac{\int_{\Omega}\left(\left|\nabla_{A} u\right|^{2}+\kappa|u|^{2}\right) \mathrm{d} x}{\left(\int_{\Omega}|u|^{p} \mathrm{~d} x\right)^{\frac{2}{p}}} \leq\left(\int_{\Omega}\left(\left|\nabla_{A} u\right|^{2}+\kappa|u|^{2}\right) \mathrm{d} x\right)^{\frac{p-2}{p}} .
$$

Portanto, por (2.1) e tomando o ínfimo em $M_{\kappa, p, \Omega}$, obtemos

$$
\left(\frac{1}{2}-\frac{1}{p}\right) m_{A}(\kappa, p, \Omega)^{\frac{p}{p-2}} \leq b_{\kappa, p, \Omega}
$$

Reciprocamente, dado $u \in E_{A} \backslash\{0\}$, existe único $t>0$ tal que $t u \in M_{\kappa, p, \Omega}$. Então

$$
\int_{\Omega}\left(\left|\nabla_{A} u\right|^{2}+\kappa|u|^{2}\right) \mathrm{d} x=t^{p-2} \int_{\Omega}|u|^{p} \mathrm{~d} x .
$$

Em particular,

$$
t^{2}=\left(\frac{\int_{\Omega}\left(\left|\nabla_{A} u\right|^{2}+\kappa|u|^{2}\right) \mathrm{d} x}{\int_{\Omega}|u|^{p} \mathrm{~d} x}\right)^{\frac{2}{p-2}}
$$

Usando (2.3) e (2.4),

$$
\begin{aligned}
b_{\kappa, p, \Omega} & \leq I_{\kappa, p, \Omega}(t u)=\frac{t^{2}}{2} \int_{\Omega}\left(\left|\nabla_{A} u\right|^{2}+\kappa|u|^{2}\right) \mathrm{d} x-\frac{t^{p}}{p} \int_{\Omega}|u|^{p} \mathrm{~d} x \\
& =t^{2}\left(\frac{1}{2}-\frac{1}{p}\right) \int_{\Omega}\left(\left|\nabla_{A} u\right|^{2}+\kappa|u|^{2}\right) \mathrm{d} x \\
& =\left(\frac{1}{2}-\frac{1}{p}\right)\left(\frac{\int_{\Omega}\left(\left|\nabla_{A} u\right|^{2}+\kappa|u|^{2}\right) \mathrm{d} x}{\left(\int_{\Omega}|u|^{p} \mathrm{~d} x\right)^{\frac{2}{p}}}\right)^{\frac{p}{p-2}}
\end{aligned}
$$

Dessa forma, tomando o ínfimo em $E_{A} \backslash\{0\}$, concluímos que

$$
b_{\kappa, p, \Omega} \leq\left(\frac{1}{2}-\frac{1}{p}\right) m_{A}(\kappa, p, \Omega)^{\frac{p}{p-2}}
$$


e o resultado segue por (2.2).

Como consequência do Lema 2.8, temos

$$
b_{\kappa, 2^{*}, \Omega}=\frac{1}{N} S_{A, \kappa^{\frac{N}{2}}} .
$$

Definamos também

$$
m(\kappa, p, \Omega):=\inf _{u \in H_{0}^{1}(\Omega) \backslash\{0\}} \frac{\int_{\Omega}\left(|\nabla u|^{2}+\kappa u^{2}\right) \mathrm{d} x}{\left(\int_{\Omega}|u|^{p} \mathrm{~d} x\right)^{\frac{2}{p}}}
$$

e ponhamos

$$
S_{\kappa}:=m\left(\kappa, 2^{*}, \Omega\right)
$$

Em particular, $S:=m\left(0,2^{*}, \Omega\right)$.

O próximo lema versa sobre a independência de $S_{A, \kappa}$ em relação ao parâmetro $\kappa \geq 0$.

Lema 2.9. Para todo $\kappa \geq 0$, temos $S_{A, \kappa}=S_{\kappa}=S$, onde $S$ é a melhor constante da imersão $H_{0}^{1}(\Omega, \mathbb{R}) \hookrightarrow L^{2^{*}}(\Omega, \mathbb{R})$. Em particular, $S_{A, \kappa}$ e $S_{\kappa}$ não dependem de $\kappa \geq 0$ nem do domínio no qual são definidos.

\section{Demonstração.}

Primeiramente, vejamos que $S_{A}=S$. Pela desigualdade diamagnética, $S_{A} \geq S$. Para a desigualdade contrária, considere $x_{0} \in \Omega$. Então existe $\delta>0$ tal que $B_{\delta}\left(x_{0}\right) \subset \Omega$. Considere também $\phi \in C_{c}^{\infty}\left(\mathbb{R}^{N}\right)$ tal que

$$
\phi \equiv 1 \mathrm{em} B_{\frac{\delta}{3}}(0), \phi \equiv 0 \mathrm{em} \mathbb{R}^{N} \backslash B_{\frac{2 \delta}{3}}(0), \phi \in[0,1], \forall x \in \mathbb{R}^{N} .
$$

e

$$
U_{\epsilon, x_{0}}(x):=\epsilon^{\frac{2-N}{2}} U\left(\frac{x-x_{0}}{\epsilon}\right), \quad \forall x \in \mathbb{R}^{N}
$$

onde

$$
U(x)=\frac{c_{N}}{\left(|x|^{2}+1\right)^{\frac{N-2}{2}}}, \quad \forall x \in \mathbb{R}^{N},
$$

e $c_{N}$ tal que $-\Delta U=U^{2^{*}}$ em $\mathbb{R}^{N}$. Vale ressaltar que a família $\left\{U_{\epsilon}\right\}_{\epsilon \geq 0}$ atinge $S$ (ver [43]).

Definamos

$$
v_{\epsilon}=\frac{u_{\epsilon}}{\left|u_{\epsilon}\right|_{2^{*}}}, \quad u_{\epsilon}=\phi\left(.-x_{0}\right) U_{\epsilon, x_{0}} \in E_{A}
$$

Pela definição de $S_{A}$,

$$
S_{A} \leq \frac{\int_{\Omega}\left|\nabla_{A}\left(e^{i \tau_{x_{0}}} v_{\epsilon}\right)\right|^{2} \mathrm{~d} x}{\left(\int_{\Omega}\left|e^{i \tau_{x_{0}}} v_{\epsilon}\right|^{2^{*}} \mathrm{~d} x\right)^{\frac{2}{2^{*}}}}
$$


onde $\tau_{x_{0}}(x)=\sum_{j=1}^{N} A^{j}\left(x_{0}\right) x^{j}, x \in \Omega$.

Mostremos que o lado direito da desigualdade acima converge para $S$ quando $\epsilon \rightarrow 0$.

Inicialmente, usando a definição de $v_{\epsilon}$ e $\tau_{x_{0}}$, observemos que

$$
\begin{aligned}
\int_{\Omega}\left|\nabla_{A}\left(e^{i \tau_{0}} v_{\epsilon}\right)\right|^{2} \mathrm{~d} x & =\int_{\Omega}\left|\nabla v_{\epsilon}\right|^{2} \mathrm{~d} x+\int_{\Omega}\left|A\left(x_{0}\right)-A(x)\right|^{2} v_{\epsilon}{ }^{2} \mathrm{~d} x \\
& =\left\|v_{\epsilon}\right\|^{2}+\int_{\Omega}\left|A\left(x_{0}\right)-A(x)\right|^{2} v_{\epsilon}{ }^{2} \mathrm{~d} x .
\end{aligned}
$$

Notemos que

$$
\int_{\Omega}\left|v_{\epsilon}\right|^{q} \mathrm{~d} x=o_{\epsilon}(1), \quad \text { para todo } 2 \leq q<2^{*}
$$

Com efeito, temos

$$
\int_{\Omega}\left|v_{\epsilon}\right|^{q} \mathrm{~d} x=\epsilon^{N+\frac{2-N}{2}} \frac{\int_{B_{\frac{2 \delta}{3}}(0)} \phi^{q}(\epsilon x) U^{q}(x) \mathrm{d} x}{\left(\int_{B_{\frac{2 \delta}{3}}(0)} \phi^{2^{*}}(\epsilon x) U^{2^{*}}(x) \mathrm{d} x\right)^{\frac{q}{2^{*}}}} .
$$

Mais ainda, $N+\frac{2-N}{2} q>0$ se, e só se, $q<2^{*}$, e $U \in D^{1,2}\left(\mathbb{R}^{N}, \mathbb{R}\right)$, donde $U \in L_{\text {loc }}^{p}\left(\mathbb{R}^{N}, \mathbb{R}\right)$, para todo $p \in\left[1,2^{*}\right]$. Dessa forma, pelo Teorema da Convergência Dominada de Lebesgue, quando $\epsilon \rightarrow 0$,

$$
\begin{aligned}
\lim _{\epsilon \rightarrow 0} \int_{\Omega}\left|v_{\epsilon}\right|^{q} \mathrm{~d} x & =\lim _{\epsilon \rightarrow 0} \epsilon^{N+\frac{2-N}{2} q} \frac{\int_{B_{\frac{2 \delta}{3}}(0)} \phi^{q}(\epsilon x) U^{q}(x) \mathrm{d} x}{\left(\int_{B_{\frac{2 \delta}{3}}(0)} \phi^{2^{*}}(\epsilon x) U^{2^{*}}(x) \mathrm{d} x\right)^{\frac{q}{2^{*}}}} \\
& =0 \cdot \frac{|U|_{2^{*}, B_{\frac{2 \delta}{3}}(0)}^{q}}{|U|_{q, B_{\frac{2 \delta}{3}}(0)}^{q}}=0 .
\end{aligned}
$$

Observe também que

$$
\left\|v_{\epsilon}\right\|^{2} \rightarrow S, \text { quando } \epsilon \rightarrow 0
$$

De fato, usando a definição de $u_{\epsilon}$, quando $\epsilon \rightarrow 0$, temos

$$
\begin{aligned}
\left\|u_{\epsilon}\right\|^{2} & =\int_{\frac{1}{\epsilon}\left(\Omega-x_{0}\right)}\left[\epsilon^{2}|(\nabla \phi)(\epsilon x)|^{2} U^{2}(x)+|\nabla U(x)|^{2} \phi^{2}(\epsilon x)\right] \mathrm{d} x \rightarrow \int_{\mathbb{R}^{N}}|\nabla U|^{2} \mathrm{~d} x, \\
\left|u_{\epsilon}\right|_{2}^{2} & =\int_{\frac{1}{\epsilon}\left(\Omega-x_{0}\right)} \epsilon^{2} \phi^{2}(\epsilon x) U^{2}(x) \mathrm{d} x \rightarrow 0 . \int_{\mathbb{R}^{N}} U^{2} \mathrm{~d} x=0 \\
\left|u_{\epsilon}\right|_{2^{*}}^{2^{*}} & =\int_{\frac{1}{\epsilon}\left(\Omega-x_{0}\right)} \phi^{2^{*}}(\epsilon x) U^{2^{*}}(x) \mathrm{d} x \rightarrow \int_{\mathbb{R}^{N}} U^{2^{*}} \mathrm{~d} x
\end{aligned}
$$


Logo, por (2.9) e (2.11) e usando o fato de a família $\left\{U_{\epsilon}\right\}_{\epsilon \geq 0}$ atingir $S$, obtemos

$$
\lim _{\epsilon \rightarrow 0}\left\|v_{\epsilon}\right\|^{2}=\lim _{\epsilon \rightarrow 0} \frac{\left\|u_{\epsilon}\right\|^{2}}{\left|u_{\epsilon}\right|_{2^{*}}^{2^{*}}}=\frac{\int_{\mathbb{R}^{N}}|\nabla U|^{2} \mathrm{~d} x}{\int_{\mathbb{R}^{N}} U^{2^{*}} \mathrm{~d} x}=S,
$$

o que verifica $(2.8)$.

Por (2.6),

$$
S_{A} \leq \frac{\int_{\Omega}\left|\nabla v_{\epsilon}\right|^{2} \mathrm{~d} x+\int_{\Omega}\left|A\left(x_{0}\right)-A(x)\right|^{2} v_{\epsilon}^{2} \mathrm{~d} x}{\left(\int_{\Omega}\left|v_{\epsilon}\right|^{2^{*}} \mathrm{~d} x\right)^{\frac{2}{2^{*}}}}=\left\|v_{\epsilon}\right\|^{2}+\int_{\Omega}\left|A\left(x_{0}\right)-A(x)\right|^{2} v_{\epsilon}^{2} \mathrm{~d} x .
$$

Fazendo $\epsilon \rightarrow 0$ e usando (2.8), encontramos $S_{A} \leq S$, donde $S_{A}=S$. Como consequência da igualdade, $S_{A}$ não depende do domínio no qual é definido, uma vez que $S$ tem essa propriedade.

Quanto à independência de $S$ em relação à $\kappa \geq 0$, usando a definição de $S_{\kappa}$ e (2.9)-(2.11), temos

$$
S \leq S_{\kappa} \leq \lim _{\epsilon \rightarrow 0} \frac{\int_{\Omega}\left(\left|\nabla u_{\epsilon}\right|^{2}+\kappa\left|u_{\epsilon}\right|^{2}\right) \mathrm{d} x}{\left(\int_{\Omega}\left|u_{\epsilon}\right|^{2^{*}} \mathrm{~d} x\right)^{\frac{2}{2^{*}}}}=S .
$$

Analogamente, usando também a desigualdade diamagnética,

$$
\begin{aligned}
S \leq S_{A, \kappa} & \leq \lim _{\epsilon \rightarrow 0} \frac{\int_{\Omega}\left[\left|\nabla_{A}\left(e^{i \tau_{x_{0}}} v_{\epsilon}\right)\right|^{2}+\kappa\left|e^{i \tau_{x_{0}}} v_{\epsilon}\right|^{2}\right] \mathrm{d} x}{\left(\int_{\Omega}\left|e^{i \tau_{x_{0}}} v_{\epsilon}\right|^{2^{*}} \mathrm{~d} x\right)^{\frac{2}{2^{*}}}} \\
& =\lim _{\epsilon \rightarrow 0} \int_{\Omega}\left(\left|\nabla v_{\epsilon}\right|^{2}+\left|A\left(x_{0}\right)-A(x)\right|^{2}\left|v_{\epsilon}\right|^{2}+\kappa\left|v_{\epsilon}\right|^{2}\right) \mathrm{d} x=S .
\end{aligned}
$$

Por (2.12) e (2.13), $S=S_{A, \kappa}=S_{\kappa}$, donde $S$ e $S_{A}$ não dependem de $\kappa \geq 0$.

Lema 2.10. Para cada $\kappa \geq 0$ e $\mathcal{D} \subset \mathbb{R}^{N}$ limitado, vale

$$
\lim _{p \rightarrow 2^{*}} b_{\kappa, p, \mathcal{D}}=b_{2^{*}} .
$$

\section{Demonstração.}

Sejam $\kappa \geq 0$ e $\mathcal{D} \subset \mathbb{R}^{N}$ limitado quaisquer. Vejamos que os limites inferior e superior de $\left(b_{\kappa, p, \mathcal{D}}\right)_{p}$ existem e coincidem.

Sejam $2 \leq p<q \leq 2^{*}$ e $u \in E_{A}(\mathcal{D})$, onde $E_{A}(\mathcal{D})$ é o espaço de Hilbert real definido como o fechamento de $C_{c}^{\infty}(\mathcal{D}, \mathbb{C})$ relativamente à norma induzida pelo produto interno

$$
\langle u, v\rangle_{A}:=\operatorname{Re}\left\{\int_{\Omega} \nabla_{A} u \overline{\nabla_{A} v} \mathrm{~d} x\right\} .
$$


Temos

$$
|u|_{p, \Omega} \leq|\mathcal{D}|^{\frac{q-p}{q p}}|u|_{q, \mathcal{D}}
$$

Logo

$$
\frac{\int_{\mathcal{D}}\left(\left|\nabla_{A} u\right|^{2}+\kappa|u|^{2}\right) \mathrm{d} x}{|u|_{p, \mathcal{D}}^{2}} \geq|\mathcal{D}|^{\frac{-2(q-p)}{q p}} \frac{\int_{\mathcal{D}}\left(\left|\nabla_{A} u\right|^{2}+\kappa|u|^{2}\right) \mathrm{d} x}{|u|_{q, \mathcal{D}}^{2}} .
$$

Portanto, fazendo $q=2^{*}$ e tomando o ínfimo em $u \in E_{A}(\mathcal{D}) \backslash\{0\}$,

$$
m_{A}(\kappa, p, \mathcal{D}) \geq|\mathcal{D}|^{\frac{-2\left(2^{*}-p\right)}{2^{*} p}} S_{A}
$$

Por outro lado, fazendo $p=2, q=p$ e procedendo como anteriormente,

$$
m_{A}(\kappa, p, \mathcal{D}) \leq|\mathcal{D}|^{\frac{p-2}{p}} m_{A}(\kappa, 2, \mathcal{D})
$$

Assim, por $(2.14)$ e $(2.15),\left(m_{A}(\kappa, p, \mathcal{D})\right)_{p}$ é uma sequência limitada, donde existem $M:=\limsup _{p \rightarrow 2^{*}} m_{A}(\kappa, p, \mathcal{D})$ e $m:=\liminf _{p \rightarrow 2^{*}} m_{A}(\kappa, p, \mathcal{D})$, limites superior e inferior de $\left(m_{A}(\kappa, p, \mathcal{D})\right)_{p}$, respectivamente.

Mostremos que $M=S_{A}=m$.

Notemos que, por (2.14),

$$
m \geq \liminf _{p \rightarrow 2^{*}}|\mathcal{D}|^{\frac{-2\left(2^{*}-p\right)}{2^{*} p}} S_{A}=S_{A} .
$$

Suponhamos por contradição que $m>S_{A}$ e seja $\epsilon \in\left(0, m-S_{A}\right)$.

Pela definição de $S_{A}$, existe $\bar{u} \in E_{A}(\mathcal{D})$ tal que

$$
\frac{\int_{\mathcal{D}}\left(\left|\nabla_{A} \bar{u}\right|^{2}+\kappa|\bar{u}|^{2}\right) \mathrm{d} x}{\left(\int_{\mathcal{D}}|\bar{u}|^{2^{*}} \mathrm{~d} x\right)^{\frac{2}{2^{*}}}}<S_{A}+\frac{\epsilon}{2} .
$$

Por outro lado, a função $p \mapsto|\bar{u}|_{p, \mathcal{D}}$ é contínua, donde existe $\bar{p} \in\left(2,2^{*}\right)$ tal que, para todo $p \in\left[\bar{p}, 2^{*}\right)$,

$$
\left|\frac{\int_{\mathcal{D}}\left(\left|\nabla_{A} \bar{u}\right|^{2}+\kappa|\bar{u}|^{2}\right) \mathrm{d} x}{\left(\int_{\mathcal{D}}|\bar{u}|^{p} \mathrm{~d} x\right)^{\frac{2}{p}}}-\frac{\int_{\mathcal{D}}\left(\left|\nabla_{A} \bar{u}\right|^{2}+\kappa|\bar{u}|^{2}\right) \mathrm{d} x}{\left(\int_{\mathcal{D}}|\bar{u}|^{2^{*}} \mathrm{~d} x\right)^{\frac{2}{2^{*}}}}\right|<\frac{\epsilon}{2} .
$$

Portanto, para todo $p \in\left[\bar{p}, 2^{*}\right]$, lembrando a forma como $\epsilon$ foi tomado,

$$
\begin{aligned}
m_{A}(\kappa, p, \mathcal{D}) & \leq \frac{\int_{\mathcal{D}}\left(\left|\nabla_{A} \bar{u}\right|^{2}+\kappa|\bar{u}|^{2}\right) \mathrm{d} x}{\left(\int_{\mathcal{D}}|\bar{u}|^{p} \mathrm{~d} x\right)^{\frac{2}{p}}}<\frac{\int_{\mathcal{D}}\left(\left|\nabla_{A} \bar{u}\right|^{2}+\kappa|\bar{u}|^{2}\right) \mathrm{d} x}{\left(\int_{\mathcal{D}}|\bar{u}|^{2^{*}} \mathrm{~d} x\right)^{\frac{2}{2^{*}}}+\frac{\epsilon}{2}} \\
& <S_{A}+\epsilon \\
& <m
\end{aligned}
$$


isto é,

$$
m=\liminf _{p \rightarrow 2^{*}} m_{A}(\kappa, p, \mathcal{D})<S_{A}+\epsilon<m,
$$

o que é uma contradição. Assim $S_{A}=m$.

Analogamente, temos $S_{A}=M$ e o resultado está mostrado.

Consideremos, para cada $\kappa \geq 0, p \in\left(2,2^{*}\right)$, o funcional

$$
J_{\kappa, p, \Omega}(u)=\frac{1}{2} \int_{\Omega}\left(|\nabla u|^{2}+\kappa u^{2}\right) \mathrm{d} x-\frac{1}{p} \int_{\Omega}|u|^{p} \mathrm{~d} x, \quad \forall u \in H_{0}^{1}(\Omega),
$$

com Variedade de Nehari

$$
N_{\kappa, p, \Omega}:=\left\{u \in H_{0}^{1}(\Omega) \backslash\{0\} ; J_{\kappa, p, \Omega}^{\prime}(u) u=0\right\} .
$$

Definamos também

$$
c_{\kappa, p, \Omega}=\inf _{u \in N_{\kappa, p, \Omega}} J_{\kappa, p, \Omega}(u) .
$$

Procedendo como nas demonstrações dos Lema 2.8 e Proposição 2.10, temos, para cada $\kappa \geq 0, \mathcal{D} \subset \mathbb{R}^{N}$ limitado,

$$
c_{\kappa, p, \mathcal{D}}=\left(\frac{1}{2}-\frac{1}{p}\right) m(\kappa, p, \mathcal{D})^{\frac{p}{p-2}} \quad \text { e } \quad \lim _{p \rightarrow 2^{*}} c_{\kappa, p, \mathcal{D}}=c_{\kappa, 2^{*}, \mathcal{D}} .
$$

Em particular,

$$
c_{\kappa, 2^{*}, \mathcal{D}}=\frac{1}{N} S^{\frac{N}{2}}=: c_{2^{*}}
$$

Logo, pelo Lema 2.9,

$$
c_{2^{*}}=b_{2^{*}}, \text { ou, equivalentemente, } \lim _{p \rightarrow 2^{*}} c_{\kappa, p, \mathcal{D}}=\lim _{p \rightarrow 2^{*}} b_{\kappa, p, \mathcal{D}}=\frac{1}{N} S^{\frac{N}{2}} .
$$

\subsection{Preliminares}

Suporemos, sem perda de generalidade, que $0 \in \Omega$. Fixemos $r>0$ tais que $B_{r}(0) \subset \Omega$ e os conjuntos

$$
\Omega^{+}:=\left\{x \in \mathbb{R}^{N} ; \operatorname{dist}(x, \Omega) \leq r\right\} \quad \text { e } \quad \Omega^{-}:=\{x \in \Omega ; \operatorname{dist}(x, \partial \Omega) \geq r\}
$$

sejam homotopicamente equivalentes a $\Omega$. Definamos de maneira análoga os entes $\left(I_{\kappa, p, r} ; M_{\kappa, p, r} ; b_{\kappa, p, r}\right)$ e $\left(J_{\kappa, p, r} ; N_{\kappa, p, r} ; c_{\kappa, p, r}\right)$, apenas trocando $\Omega$ pela bola $B_{r}(0) \subset \Omega$. Consideremos a aplicação $\Phi_{\kappa, p}: \Omega_{r}^{-} \rightarrow M_{\kappa, p, \Omega}$ dada por

$$
\left[\Phi_{\kappa, p}(y)\right](x)= \begin{cases}t_{\kappa, p, y} e^{i \tau_{y}(x)} u_{\kappa, p, r}(|x-y|), & x \in B_{r}(y), \\ 0, & x \in \Omega \backslash B_{r}(y) .\end{cases}
$$

onde $\tau_{y}(x):=\sum_{j=1}^{N} A^{j}(y) x^{j}, x=\left(x_{1}, \ldots, x_{N}\right) \in \Omega, u_{\kappa, p, r} \in N_{\kappa, p, r}$ atinge $c_{\kappa, p, r}$ e é, argumentando como na Seção 3 do Capítulo 1, positiva e radialmente simétrica, e $t_{\kappa, p, y}>0$ é tal que $t_{\kappa, p, y} e^{i \tau_{y}} u_{\kappa, p, r}(|.-y|) \in M_{\kappa, p, \Omega}$. 
Lema 2.11. Para $\kappa \geq 0$ fixado,

$$
\lim _{p \rightarrow 2^{*}} \max _{y \in \Omega_{r}^{-}}\left|\Phi_{\kappa, p}(y)-c_{2^{*}}\right|=0
$$

\section{Demonstração.}

Aplicaremos o mesmo argumento apresentado no Lema 1.13. Dessa forma, mostremos que, fixado $\kappa \geq 0$ e dadas $\left(p_{n}\right) \subset \mathbb{R} \operatorname{com} p_{n} \rightarrow 2^{*}$ e $\left(y_{n}\right) \subset \Omega_{r}^{-}$quaisquer,

$$
I_{\kappa, p_{n}, \Omega}\left(\Phi_{\kappa, p_{n}}\left(y_{n}\right)\right) \rightarrow c_{2^{*}}, \text { quando } n \rightarrow \infty .
$$

Primeiramente, notemos que, fazendo $t_{\kappa, p_{n}, y_{n}}=: t_{n}, I_{\kappa, p_{n}, \Omega}=: I_{n}, \Phi_{\kappa, p_{n}}\left(y_{n}\right)=: \Phi_{n}\left(y_{n}\right)$, $u_{\kappa, p_{n}, r}=: u_{n}$,

$$
\begin{aligned}
I_{n}\left(\Phi_{n}\left(y_{n}\right)\right)= & \frac{1}{2} \int_{\Omega}\left(\left|\nabla_{A} \Phi_{n}\left(y_{n}\right)\right|^{2}+\kappa\left|\Phi_{n}\left(y_{n}\right)\right|^{2}\right) \mathrm{d} x-\frac{1}{p_{n}} \int_{\Omega}\left|\Phi_{n}\left(y_{n}\right)\right|^{p_{n}} \mathrm{~d} x \\
= & \frac{t_{n}^{2}}{2} \int_{B_{r}(0)}\left|A\left(z+y_{n}\right)-A\left(y_{n}\right)\right|^{2} u_{n}^{2} \mathrm{~d} x+ \\
& +\frac{t_{n}^{2}}{2} \int_{B_{r}(0)}\left(\left|\nabla u_{n}\right|^{2}+\kappa u_{n}^{2}\right) \mathrm{d} x-\frac{t_{n} p_{n}}{p_{n}} \int_{B_{r}(0)} u_{n}^{p_{n}} \mathrm{~d} x \\
= & \frac{t_{n}^{2}}{2} \int_{B_{r}(0)}\left|A\left(z+y_{n}\right)-A\left(y_{n}\right)\right|^{2} u_{n}^{2} \mathrm{~d} x+J_{\kappa, p_{n}, B_{r}(0)}\left(t_{n} u_{n}\right) \\
\leq & \frac{t_{n}^{2}}{2} \int_{B_{r}(0)}\left|A\left(z+y_{n}\right)-A\left(y_{n}\right)\right|^{2} u_{n}{ }^{2} \mathrm{~d} x+J_{\kappa, p_{n}, r}\left(u_{n}\right) \\
= & \frac{t_{n}{ }^{2}}{2} \int_{B_{r}(0)}\left|A\left(z+y_{n}\right)-A\left(y_{n}\right)\right|^{2} u_{n}{ }^{2} \mathrm{~d} x+c_{\kappa, p_{n}, r} .
\end{aligned}
$$

Além disso, pela forma como $t_{n}$ foi tomado e pela desigualdade diamagnética,

$$
\begin{aligned}
I_{n}\left(\Phi_{n}\left(y_{n}\right)\right) \geq & I_{n}\left(e^{i \tau_{y_{n}}} u_{n}\left(.-y_{n}\right)\right) \\
\geq & \frac{1}{2} \int_{\Omega}\left(|\nabla| e^{i \tau_{y_{n}}} u_{n}\left(.-y_{n}\right)||^{2}+\left|e^{i \tau_{y_{n}}} u_{n}\left(.-y_{n}\right)\right|^{2}\right) \mathrm{d} x- \\
& -\frac{1}{p} \int_{\Omega}\left|e^{i \tau_{y_{n}}} u_{n}\left(.-y_{n}\right)\right|^{p} \mathrm{~d} x \\
= & J_{\kappa, p_{n}, r}\left(u_{n}\right)=c_{\kappa, p_{n}, r} .
\end{aligned}
$$

Portanto, tendo em vista (2.17), nosso objetivo é mostrar que

$$
\frac{t_{n}^{2}}{2} \int_{B_{r}(0)}\left|A\left(z+y_{n}\right)-A\left(y_{n}\right)\right|^{2} u_{n}^{2} \mathrm{~d} x=o_{n}(1) .
$$

Vejamos que $u_{n} \rightarrow 0$ em $H_{0}^{1}\left(B_{r}(0), \mathbb{R}\right)$ e $\left(t_{n}\right)_{n}$ é limitada.

Como $u_{n} \in N_{\kappa, p_{n}, r}$ atinge $c_{\kappa, p_{n}, r}$,

$$
\int_{B_{r}(0)}\left(\left|\nabla u_{n}\right|^{2}+\kappa u_{n}^{2}\right) \mathrm{d} x=\left(\frac{1}{2}-\frac{1}{p_{n}}\right)^{-1} c_{\kappa, p_{n}, r} .
$$


Dessa forma, por $(2.17),\left(u_{n}\right) \subset H_{0}^{1}\left(B_{r}(0), \mathbb{R}\right)$ é uma sequência limitada. Logo existe $v \in H_{0}^{1}\left(B_{r}(0)\right)$ tal que

$$
\left\{\begin{array}{l}
u_{n} \rightarrow v \text { em } H_{0}^{1}\left(B_{r}(0), \mathbb{R}\right), \text { quando } n \rightarrow \infty \\
u_{n} \rightarrow v \text { em } L^{s}\left(B_{r}(0), \mathbb{R}\right), \text { para todo } s \in\left[1,2^{*}\right), \text { quando } n \rightarrow \infty \\
u_{n}(x) \rightarrow v(x) \text { quase sempre em } B_{r}(0), \text { quando } n \rightarrow \infty
\end{array}\right.
$$

Pelo fato de $u_{n} \in N_{\kappa, p_{n}, r}$ atingir $c_{\kappa, p_{n}, r}, u_{n}$ é solução de

$$
\left\{\begin{array}{l}
-\Delta u+\kappa u=u^{p_{n}-1} \text { em } B_{r}(0) \\
u=0 \text { em } \partial B_{r}(0)
\end{array}\right.
$$

Logo, para qualquer $\psi \in C_{c}^{\infty}\left(B_{r}(0)\right)$,

$$
\int_{B_{r}(0)}\left(\nabla u_{n} \nabla \psi+\kappa u_{n} \psi\right) \mathrm{d} x=\int_{B_{r}(0)} u_{n}^{p-1} \psi \mathrm{d} x .
$$

Por (2.20), quando $n \rightarrow \infty$,

$$
\int_{B_{r}(0)}\left(\nabla u_{n} \nabla \psi+\kappa u_{n} \psi\right) \mathrm{d} x \rightarrow \int_{B_{r}(0)}(\nabla v \nabla \psi+\kappa v \psi) \mathrm{d} x .
$$

Vejamos que

$$
\int_{B_{r}(0)} u_{n}^{p_{n}-1} \psi \mathrm{d} x \rightarrow \int_{B_{r}(0)} v^{2^{*}-1} \psi \mathrm{d} x, \quad \text { quando } n \rightarrow \infty .
$$

Usaremos o Teorema da Convergência Dominada de Lebesgue Generalizado. É fácil ver que, quando $n \rightarrow \infty$,

$$
u_{n}{ }^{p_{n}-1}(x) \psi(x) \rightarrow v^{2^{*}-1}(x) \psi(x) \text { quase sempre em } B_{r}(0) .
$$

Da convergência forte de $u_{n}$ para $v$ em $L^{s}\left(B_{r}(0), \mathbb{R}\right)$, para cada $s \in\left[1,2^{*}\right)$, existe $h_{s} \in L^{s}\left(B_{r}(0), \mathbb{R}\right)$ tal que, a menos de subsequência, $u_{n} \leq h_{s}$ quase sempre em $B_{r}(0)$. Para $s_{0} \in\left[1,2^{*}\right)$ a ser escolhido adequadamente, definamos $H_{s_{0}}:=\max \left\{h_{s_{0}}, 1\right\}$. Mostremos que $H_{s_{0}}{ }^{p_{n}-1} \rightarrow{H_{s_{0}}}^{2^{*}-1}$ em $L^{1}\left(B_{r}(0), \mathbb{R}\right)$. Pelo Teorema do Valor Médio, existe $\theta\left(p_{n}, x\right)=: \theta_{n}(x) \in[0,1]$ tal que

$$
\begin{gathered}
\int_{B_{r}(0)}\left|H_{s_{0}} p_{n}-1-H_{s_{0}} 2^{*}-1\right| \mathrm{d} x=\int_{B_{r}(0)}\left|\exp \left[\left(p_{n}-1\right) \ln H_{s_{0}}\right]-\exp \left[\left(2^{*}-1\right) \ln H_{s_{0}}\right]\right| \mathrm{d} x \\
\leq \int_{B_{r}(0)}\left(2^{*}-p_{n}\right) \ln H_{s_{0}} \exp \left\{\left[\left(p_{n}-1\right)+\theta_{n}(x)\left(2^{*}-p_{n}\right)\right] \ln H_{s_{0}}\right\} \mathrm{d} x
\end{gathered}
$$

Além disso, como $\ln H_{s_{0}} \geq 0$, para $n$ suficientemente grande e $\alpha>0$ tal que $2^{*}-1+\alpha<2^{*}$, temos

$$
0 \leq\left[\left(p_{n}-1\right)+\theta_{n}(x)\left(2^{*}-p_{n}\right)\right] \ln H_{s_{0}} \leq\left[2^{*}-1+\alpha\right] \ln H_{s_{0}} .
$$


Logo

$$
\begin{aligned}
\int_{B_{r}(0)}\left|H_{s_{0}} p_{n}-1-H_{s_{0}}{ }^{2^{*}-1}\right| \mathrm{d} x & \leq \int_{B_{r}(0)}\left(2^{*}-p_{n}\right) \ln H_{s_{0}} \exp \left\{\left(2^{*}-1+\alpha\right) \ln H_{s_{0}}\right\} \mathrm{d} x \\
& =\int_{B_{r}(0)} H_{s_{0}}{ }^{2^{*}-1+\alpha}\left(2^{*}-p_{n}\right) \ln H_{s_{0}} \mathrm{~d} x
\end{aligned}
$$

Tomemos $\beta>0$ de modo que $2^{*}-1+\alpha+\beta<2^{*}$. Note que existe $K>0$ tal que, para $l>K, \ln l<l^{\beta}$. Segue que

$$
\begin{aligned}
\int_{B_{r}(0)}\left|H_{s_{0}}{ }^{p_{n}-1}-H_{s_{0}}{ }^{2^{*}-1}\right| \mathrm{d} x \leq & \int_{B_{r}(0)} H_{s_{0}}{ }^{2^{*}-1+\alpha}\left(2^{*}-p_{n}\right) \ln H_{s_{0}} \mathrm{~d} x \\
= & \int_{B_{r}(0) \cap\left\{H_{s_{0}} \geq K\right\}} H_{s_{0}}{ }^{2^{*}-1+\alpha}\left(2^{*}-p_{n}\right) \ln H_{s_{0}} \mathrm{~d} x+ \\
& +\int_{B_{r}(0) \cap\left\{H_{s_{0}} \leq K\right\}} H_{s_{0}}{ }^{2^{*}-1+\alpha}\left(2^{*}-p_{n}\right) \ln H_{s_{0}} \mathrm{~d} x \\
\leq & \left(2^{*}-p_{n}\right) \int_{B_{r}(0) \cap\left\{H_{s_{0}} \geq K\right\}} H_{s_{0}}{ }^{2^{*}-1+\alpha+\beta} \mathrm{d} x+ \\
& +\left(2^{*}-p_{n}\right) \int_{B_{r}(0) \cap\left\{H_{s_{0}} \leq K\right\}} H_{s_{0}}{ }^{2^{*}-1+\alpha} K \mathrm{~d} x .
\end{aligned}
$$

Escolhamos $s_{0} \in\left(2^{*}-1+\alpha+\beta, 2^{*}\right)$. Como $B_{r}(0)$ é limitado,

$$
\int_{B_{r}(0)} H_{s_{0}}^{2^{*}-1+\alpha+\beta} \mathrm{d} x<\infty
$$

donde

$$
H_{s_{0}}{ }^{p_{n}-1} \rightarrow{H_{s_{0}}}^{2^{*}-1} \text { em } L^{1}\left(B_{r}(0), \mathbb{R}\right), \text { quando } n \rightarrow \infty
$$

Assim,

$$
u_{n}{ }^{p_{n}-1}(x) \psi(x) \rightarrow v^{2^{*}-1}(x) \psi(x) \text {, quando } n \rightarrow \infty \text {, quase sempre em } B_{r}(0),
$$

e

$$
u_{n}^{p_{n}-1} \psi \leq H_{s_{0}}^{p_{n}-1} \psi
$$

com

$$
H_{s_{0}}{ }^{p_{n}-1} \psi \rightarrow{H_{s_{0}}}^{2^{*}-1} \psi \text { em } L^{1}\left(B_{r}(0), \mathbb{R}\right) \text {, quando } n \rightarrow \infty .
$$

Portanto, pelo Teorema da Convergência Dominada de Lebesgue Generalizado,

$$
u_{n}^{p_{n}-1} \psi \rightarrow v^{2^{*}-1} \psi \text { em } L^{1}\left(B_{r}(0), \mathbb{R}\right)
$$

Por $(2.21)$ e $(2.22), v \in H_{0}^{1}\left(B_{r}(0)\right) \backslash\{0\}$ é solução do problema

$$
\left\{\begin{array}{l}
-\Delta u+\kappa u=u^{2^{*}-1}, \text { em } B_{r}(0), \\
u=0, \text { sobre } \partial B_{r}(0) .
\end{array}\right.
$$


Pelo Teorema de Brézis-Kato, $v \in H_{0}^{1}\left(B_{r}(0)\right) \cap H^{2}\left(B_{r}(0)\right)$. Portanto, pela Identidade de Pohozaev, $v \equiv 0$ em $B_{r}(0)$ e, consequentemente,

$$
u_{n} \rightarrow 0 \mathrm{em} H_{0}^{1}\left(B_{r}(0), \mathbb{R}\right)
$$

Vejamos que $\left(t_{n}\right)_{n}$ é limitada. Com efeito, basta observarmos que, pela forma como $t_{n}$ foi tomada, temos

$$
\begin{aligned}
\int_{B_{r}(0)}\left|A\left(y_{n}\right)-A\left(z+y_{n}\right)\right|^{2} u_{n}{ }^{2} \mathrm{~d} & +\int_{B_{r}(0)}\left|\nabla u_{n}\right|^{2}+\kappa u_{n}{ }^{2} \mathrm{~d} x= \\
= & \int_{\Omega}\left|A(x)-A\left(y_{n}\right)\right|^{2} u_{n}{ }^{2}\left(x-y_{n}\right) \mathrm{d} x+ \\
& +\int_{\Omega}\left[\left|\nabla u_{n}\left(x-y_{n}\right)\right|^{2}+\kappa u_{n}{ }^{2}\left(x-y_{n}\right)\right] \mathrm{d} x \\
= & \int_{\Omega}\left(\left|\nabla_{A}\left(e^{i \tau_{y}} u_{n}\left(x-y_{n}\right)\right)\right|^{2}+\kappa\left|e^{i \tau_{y}} u_{n}\left(x-y_{n}\right)\right|^{2}\right) \mathrm{d} x \\
= & t_{n}{ }^{p_{n}-2} \int_{\Omega}\left|e^{i \tau_{y}} u_{n}\left(x-y_{n}\right)\right|^{p_{n}} \mathrm{~d} x \\
= & t_{n}{ }^{p_{n}-2} \int_{\Omega}\left|u_{n}\left(x-y_{n}\right)\right|^{p_{n}} \mathrm{~d} x \\
= & t_{n}{ }^{p_{n}-2} \int_{B_{r}(0)}\left|u_{n}\right|^{p_{n}} \mathrm{~d} x
\end{aligned}
$$

Mais ainda,

$$
\int_{B_{r}(0)}\left(\left|\nabla u_{n}\right|^{2}+\kappa u_{n}^{2}\right) \mathrm{d} x=\int_{B_{r}(0)}\left|u_{n}\right|^{p_{n}} \mathrm{~d} x
$$

uma vez que $u_{n} \in N_{\kappa, p_{n}, r}$. Assim

$$
\int_{B_{r}(0)}\left|A\left(y_{n}\right)-A\left(z+y_{n}\right)\right|^{2} u_{n}^{2} \mathrm{~d} x=\left(t_{n}^{p_{n}-2}-1\right) \int_{B_{r}(0)}\left(\left|\nabla u_{n}\right|^{2}+\kappa u_{n}^{2}\right) \mathrm{d} x .
$$

Suponhamos, por contradição, que, a menos de subsequência, $t_{n} \rightarrow+\infty$ quando $n \rightarrow \infty$. Tendo em vista (2.17), (2.19), (2.23) e a igualdade anterior,

$$
\begin{aligned}
0 & =\lim _{n \rightarrow \infty} \int_{B_{r}(0)}\left|A\left(y_{n}\right)-A\left(z+y_{n}\right)\right|^{2} u_{n}{ }^{2} \mathrm{~d} x \\
& =\lim _{n \rightarrow \infty}\left(t_{n}^{p_{n}-2}-1\right) \int_{B_{r}(0)}\left(\left|\nabla u_{n}\right|^{2}+\kappa u_{n}{ }^{2}\right) \mathrm{d} x \\
& =+\infty,
\end{aligned}
$$

o que é um absurdo. Portanto $\left(t_{n}\right)_{n}$ é limitada.

Por fim, usando (2.23), as imersões de Sobolev e a limitação de $\left(t_{n}\right)_{n}$, segue (2.18). Como este argumento pode ser aplicado a quaisquer subsequências das sequências tomadas inicalmente, o resultado é válido. 


\subsection{A aplicação baricentro}

Definamos a aplicação baricentro $\beta: M_{\kappa, p, \Omega} \rightarrow \mathbb{R}^{N}$ como

$$
\beta(u)=\frac{\int_{\Omega} x \cdot|u|^{2^{*}} \mathrm{~d} x}{\int_{\Omega}|u|^{2^{*}} \mathrm{~d} x}
$$

Sobre esta aplicação, temos o seguinte resultado.

Proposição 2.12. Para $\kappa \geq 0$ fixado, existem $\epsilon=\epsilon(\kappa)>0$ e $p^{*}=p^{*}(\kappa) \in\left(2,2^{*}\right)$ tais que, para $p \in\left[p^{*}, 2^{*}\right), \beta(u) \in \Omega_{r}^{+}$, desde que $u \in M_{\kappa, p, \Omega}$ e $I_{\kappa, p, \Omega}(u) \leq \frac{1}{N} S^{\frac{N}{2}}+\epsilon$.

\section{Demonstração.}

Fixemos $\kappa \geq 0$. Primeiramente, notemos que, por (2.17), para $p$ suficientemente próximo de $2^{*}$, o conjunto

$$
\left\{u \in M_{\kappa, p, \Omega} ; I_{\kappa, p, \Omega}(u) \leq \frac{1}{N} S^{\frac{N}{2}}+\epsilon\right\}
$$

não é vazio.

Suponhamos, por contradição, que o resultado não vale. Então existem sequências $\left(p_{n}\right)_{n},\left(\epsilon_{n}\right)_{n}, \operatorname{com} p_{n} \in\left(2,2^{*}\right), p_{n} \rightarrow 2^{*}$ e $\epsilon_{n}>0, \epsilon_{n} \rightarrow 0$, e $u_{n} \in M_{\kappa, p_{n}, \Omega}, \epsilon_{n} \rightarrow 0$ tais que

$$
I_{\kappa, p_{n}, \Omega}\left(u_{n}\right) \leq \frac{1}{N} S^{\frac{N}{2}} \text { e } \beta\left(u_{n}\right) \notin \Omega_{r}^{+} .
$$

Por (2.17), temos

$$
\lim _{n \rightarrow \infty} I_{\kappa, p_{n}, \Omega}\left(u_{n}\right) \geq \lim _{n \rightarrow \infty} b_{\kappa, p_{n}, \Omega}=\frac{1}{N} S^{\frac{N}{2}}
$$

Logo, por (2.24),

$$
\lim _{n \rightarrow \infty} I_{\kappa, p_{n}, \Omega}\left(u_{n}\right)=\frac{1}{N} S^{\frac{N}{2}} .
$$

Notemos também que, como $u_{n} \in M_{\kappa, p, \Omega}$,

$$
\int_{\Omega}\left(\left|\nabla_{A} u_{n}\right|^{2}+\kappa\left|u_{n}\right|^{2}\right) \mathrm{d} x=\int_{\Omega}\left|u_{n}\right|_{n}^{p} \mathrm{~d} x,
$$

donde

$$
I_{\kappa, p_{n}, \Omega}\left(u_{n}\right)=\left(\frac{1}{2}-\frac{1}{p_{n}}\right) \int_{\Omega}\left(\left|\nabla_{A} u_{n}\right|^{2}+\kappa\left|u_{n}\right|^{2}\right) \mathrm{d} x
$$

e, por (2.25),

$$
\lim _{n \rightarrow \infty} \int_{\Omega}\left(\left|\nabla_{A} u_{n}\right|^{2}+\kappa\left|u_{n}\right|^{2}\right) \mathrm{d} x=S^{\frac{N}{2}} .
$$

Daí, quando $n \rightarrow \infty$,

$$
\lim _{n \rightarrow \infty} \frac{\int_{\Omega}\left(\left|\nabla_{A} u_{n}\right|^{2}+\kappa\left|u_{n}\right|^{2}\right) \mathrm{d} x}{\left(\int_{\Omega}\left|u_{n}\right|^{p_{n}} \mathrm{~d} x\right)^{\frac{2}{p_{n}}}}=\lim _{n \rightarrow \infty}\left(\int_{\Omega}\left(\left|\nabla_{A} u_{n}\right|^{2}+\kappa\left|u_{n}\right|^{2}\right) \mathrm{d} x\right)^{1-\frac{2}{p_{n}}}=S .
$$


Pela desigualdade diamagnética e o limite anterior

$$
\lim _{n \rightarrow \infty} \frac{\int_{\Omega}\left(|\nabla| u_{n}||^{2}+\kappa\left|u_{n}\right|^{2}\right) \mathrm{d} x}{\left(\int_{\Omega}\left|u_{n}\right|^{p_{n}} \mathrm{~d} x\right)^{\frac{2}{p_{n}}}} \leq \lim _{n \rightarrow \infty} \frac{\int_{\Omega}\left(\left|\nabla_{A} u_{n}\right|^{2}+\kappa\left|u_{n}\right|^{2}\right) \mathrm{d} x}{\left(\int_{\Omega}\left|u_{n}\right|^{p_{n}} \mathrm{~d} x\right)^{\frac{2}{p_{n}}}}=S
$$

O limite (2.26) implica que, para $\delta_{1}>0$ a ser escolhido posteriormente, existem $n_{\delta_{1}} \in \mathbb{N}$ tal que, para $n \geq n_{\delta_{1}}$,

$$
\frac{\int_{\Omega}\left(|\nabla| u_{n}||^{2}+\kappa\left|u_{n}\right|^{2}\right) \mathrm{d} x}{\left(\int_{\Omega}\left|u_{n}\right|^{p_{n}} \mathrm{~d} x\right)^{\frac{2}{p_{n}}}} \leq S+\delta_{1} .
$$

Argumentando como em (2.16) no Lema 2.10, para $\delta_{2}>0$ também a ser escolhido posteriormente, existe $n_{\delta_{2}} \in \mathbb{N}$ tal que, para todo $n \geq n_{\delta_{2}}$,

$$
\frac{\int_{\Omega}\left(|\nabla| u_{n}||^{2}+\kappa\left|u_{n}\right|^{2}\right) \mathrm{d} x}{\left(\int_{\Omega}\left|u_{n}\right|^{2^{*}} \mathrm{~d} x\right)^{\frac{2}{2^{*}}}} \leq \frac{\int_{\Omega}\left(\left.|\nabla| u_{n}\right|^{2}+\kappa\left|u_{n}\right|^{2}\right) \mathrm{d} x}{\left(\int_{\Omega}\left|u_{n}\right|^{p_{n}} \mathrm{~d} x\right)^{\frac{2}{p_{n}}}}+\delta_{2} .
$$

Assim, segue de (2.27) e (2.28) que, para $n \geq \max _{j=1,2} n_{\delta_{j}}$,

$$
S \leq \frac{\int_{\Omega}\left(|\nabla| u_{n}||^{2}+\kappa\left|u_{n}\right|^{2}\right) \mathrm{d} x}{\left(\int_{\Omega}\left|u_{n}\right|^{2^{*}} \mathrm{~d} x\right)^{\frac{2}{2^{*}}}} \leq S+\delta_{1}+\delta_{2}
$$

Afirmação: Existe $\eta>0$ tal que

$$
v \in H_{0}^{1}(\Omega), \frac{\int_{\Omega}\left(|\nabla v|^{2}+\kappa v^{2}\right) \mathrm{d} x}{\left(\int_{\Omega}|v|^{2^{*}} \mathrm{~d} x\right)^{\frac{2}{2^{*}}}} \leq S+\eta, \quad \text { então } \beta(v) \in \Omega_{r}^{+} .
$$

Com efeito, suponha, por contradição, que isto não ocorre. Então existem $\left(v_{n}\right) \subset H_{0}^{1}(\Omega, \mathbb{R})$, $\eta_{n} \rightarrow 0$ tal que

$$
\frac{\int_{\Omega}\left(\left|\nabla v_{n}\right|^{2}+\kappa v_{n}^{2}\right) \mathrm{d} x}{\left(\int_{\Omega}\left|v_{n}\right|^{2^{*}} \mathrm{~d} x\right)^{\frac{2}{2^{*}}}} \leq S+\eta_{n}, \operatorname{com} \beta\left(v_{n}\right) \notin \Omega_{r}^{+} .
$$

Usaremos o Lema de Concentração-Compacidade de Lions (ver [43, Lemma 1.40]). Façamos $w_{n}:=\frac{v_{n}}{\left|v_{n}\right|_{2^{*}, \Omega}}$. Então $\left(w_{n}\right) \subset H_{0}^{1}(\Omega, \mathbb{R})$ é uma sequência limitada. Assim, existem $u \in H_{0}^{1}(\Omega, \mathbb{R}), \mu, \nu \in \mathcal{M}\left(\mathbb{R}^{N}\right)$, espaço das medidas de Radon finitas em $\mathbb{R}^{N}$, tal que, a menos 
de subsequência,

$$
\left\{\begin{array}{l}
\left|w_{n}\right| \rightarrow u \text { em } D^{1,2}\left(\mathbb{R}^{N}, \mathbb{R}\right), \text { quando } n \rightarrow \infty \\
\left|\nabla w_{n}-\nabla u\right|^{2} \rightarrow \mu \text { em } \mathcal{M}\left(\mathbb{R}^{N}\right), \text { quando } n \rightarrow \infty \\
\left|w_{n}-u\right|^{2^{*}} \rightarrow \nu \text { em } \mathcal{M}\left(\mathbb{R}^{N}\right), \text { quando } n \rightarrow \infty \\
w_{n}(x) \rightarrow u(x) \text { quase sempre em } \Omega, \text { quando } n \rightarrow \infty
\end{array}\right.
$$

onde, quando necessário, as funções de $H_{0}^{1}(\Omega, \mathbb{R})$ são prolongadas a $\mathbb{R}^{N}$ via extensão por zero no complementar de $\Omega$. Da limitação de $\Omega$, temos, pelo Lema de Concentração-Compacidade de Lions,

$$
S=|\nabla u|_{2}^{2}+\|\mu\|_{\mathcal{M}\left(\mathbb{R}^{N}\right)}, 1=|u|_{2^{*}}^{2^{*}}+\|\nu\|_{\mathcal{M}\left(\mathbb{R}^{N}\right)},\|\nu\|_{\mathcal{M}\left(\mathbb{R}^{N}\right)}^{\frac{2}{2^{*}}} \leq S^{-1}\|\mu\|_{\mathcal{M}\left(\mathbb{R}^{N}\right)} .
$$

Da definição de $S,\left(|u|_{2^{*}}^{2^{*}}\right)^{\frac{2}{2^{*}}} \leq S^{-1}|\nabla u|_{2}^{2}$. Logo, tendo em vista as desigualdades anteriores,

$$
1 \geq\left(|u|_{2^{*}}^{2^{*}} \frac{2}{2^{*}}+\|\nu\|_{\mathcal{M}\left(\mathbb{R}^{N}\right)}^{\frac{2}{2^{*}}}>1\right.
$$

donde $u \equiv 0$ ou $\nu \equiv 0$. Vejamos que não pode ocorrer $\nu \equiv 0$. Com feito, se $\nu \equiv 0$, então

$$
1=|u|_{2^{*}}^{2^{*}}+\|\nu\|_{\mathcal{M}\left(\mathbb{R}^{N}\right)}=|u|_{2^{*}}^{2^{*}},
$$

donde, pela definição de $S,|\nabla u|_{2}^{2} \geq S$. Por outro lado,

$$
S=|\nabla u|_{2}^{2}+\|\mu\|_{\mathcal{M}\left(\mathbb{R}^{N}\right)} \geq|\nabla u|_{2}^{2}
$$

Portanto $S=|\nabla u|_{2}^{2}$. Mas isto é uma contradição, uma vez que $S$ só é atingido em $\mathbb{R}^{N}$. Assim devemos ter $u \equiv 0$ e, consequentemente, pelo Lema de Concentração- Compacidade, $\nu$ e $\mu$ são medidas concentradas em um único ponto $y \in \mathbb{R}^{N}$ e satisfazem $\|\nu\|_{\mathcal{M}\left(\mathbb{R}^{N}\right)}^{\frac{2}{2^{*}}}=S^{-1}\|\mu\|_{\mathcal{M}\left(\mathbb{R}^{N}\right)}$. Vale ressaltar que, como $u \equiv 0$, devemos ter $\|\nu\|_{\mathcal{M}\left(\mathbb{R}^{N}\right)}=1 \mathrm{e}$ $\|\mu\|_{\mathcal{M}\left(\mathbb{R}^{N}\right)}=S$. Sobre o ponto no qual as medidas concentram-se, temos $y \in \bar{\Omega}$. De fato, suponhamos por contradição que $y \notin \bar{\Omega}$. Então existe $\delta>0$ tal que $B_{3 \delta}(y) \cap \Omega=\emptyset$. Seja $\varphi \in C_{c}^{\infty}\left(\mathbb{R}^{N}\right)$ tal que $\varphi=1$ em $B_{\delta}(y)$ e $\varphi=0$ em $B_{2 \delta}^{c}(y)$. Porém, como supp $w_{n} \subset \Omega$, usando os limites fracos em $\mathcal{M}\left(\mathbb{R}^{N}\right)$,

$$
0=\lim _{n \rightarrow \infty} \int_{\mathbb{R}^{N}}\left|w_{n}\right|^{2^{*}} \varphi \mathrm{d} x=\int_{\mathbb{R}^{N}} \varphi \mathrm{d} \nu
$$

isto é, $\mu \equiv 0$, o que é uma contradição. Por fim, sejam $\Psi: \mathbb{R}^{N} \rightarrow \mathbb{R}^{N}, \Upsilon: \mathbb{R}^{N} \rightarrow \mathbb{R}$ contínuas com suporte compacto e tais que, em uma vizinhança de $\Omega, \Psi=I d_{\mathbb{R}^{N}}$ e $\Upsilon=1$. Então, quando $n \rightarrow \infty$, tendo em vista os limites fracos em $\mathcal{M}\left(\mathbb{R}^{N}\right)$,

$$
\beta\left(v_{n}\right)=\beta\left(w_{n}\right)=\frac{\int_{\mathbb{R}^{N}} x \cdot\left|w_{n}\right|^{2^{*}} \mathrm{~d} x}{\int_{\mathbb{R}^{N}}\left|w_{n}\right|^{2^{*}} \mathrm{~d} x}=\frac{\int_{\mathbb{R}^{N}} \Psi\left|w_{n}\right|^{2^{*}} \mathrm{~d} x}{\int_{\mathbb{R}^{N}} \Upsilon\left|w_{n}\right|^{2^{*}} \mathrm{~d} x} \rightarrow \frac{\int_{\{y\}} \psi \mathrm{d} \nu}{\int_{\{y\}} \Upsilon \mathrm{d} \nu}=\frac{\nu(y) \Psi(y)}{\nu(y) \Upsilon(y)}=y \in \bar{\Omega} .
$$


Mas isto contradiz o fato de $\beta\left(v_{n}\right) \notin \Omega$. Portanto a afirmação é válida. Para finalizarmos a demonstração da proposição, basta tomarmos, em $(2.29), \delta_{1}=\delta_{2}=\frac{\eta}{2}, \eta$ dado na afirmação, e observarmos que $\beta\left(\left|u_{n}\right|\right)=\beta\left(u_{n}\right)$. Com isso, teremos

$$
\beta\left(u_{n}\right) \in \Omega_{r}^{+},
$$

o que contradiz a maneira como $\left(u_{n}\right)_{n}$ foi tomada. Esta contradição mostra o resultado.

Para encerrarmos esta seção, seja, para $\kappa \geq 0$ fixado, $\epsilon=\epsilon(\kappa)>0$ dado na Proposição 2.12.

Definamos

$$
\epsilon^{*}=\epsilon^{*}(\kappa)=\frac{1}{N} S^{\frac{N}{2}}+\epsilon
$$

e o conjunto

$$
M_{\kappa, p, \Omega}^{\epsilon^{*}}:=\left\{u \in M_{\kappa, p, \Omega} ; I_{\kappa, p, \Omega}(u) \leq \epsilon^{*}\right\} .
$$

Corolário 2.13. Para $\kappa \geq 0$ fixado, existe $\bar{p}(\kappa) \in\left(2,2^{*}\right)$ tal que, para todo $p \in\left[\bar{p}(\kappa), 2^{*}\right)$,

$$
\Phi_{\kappa, p}\left(\Omega_{r}^{-}\right) \subset M_{\kappa, p, \Omega}^{\epsilon^{*}}, \quad \beta\left(M_{\kappa, p, \Omega}^{\epsilon^{*}}\right) \subset \Omega_{r}^{+} .
$$

\section{Demonstração.}

O resultado é imediato a partir dos Lema 2.11 e Proposição 2.12 .

\subsection{Prova do Teorema 2.1}

Proposição 2.14. Para $\kappa \geq 0$ fixado, sejam $\bar{p}=\bar{p}(\kappa) \in\left(2,2^{*}\right)$ e $\epsilon=\epsilon(\kappa)>0$ dados na Proposição 2.12. Para cada $p \in\left[\bar{p}, 2^{*}\right)$,

$$
\operatorname{cat}\left(M_{\kappa, p, \Omega}^{\epsilon^{*}}\right) \geq \operatorname{cat}(\Omega)
$$

onde $\epsilon^{*}=\frac{1}{N} S^{\frac{N}{2}}+\epsilon$.

\section{Demonstração.}

Suponha que $\operatorname{cat}\left(M_{\kappa, p, \Omega}^{\epsilon^{*}}\right)=k$. Então existem conjuntos $\Upsilon_{j} \subset O_{\kappa}, j=1, \ldots, k$, conjuntos fechados e contráteis em $M_{\kappa, p, \Omega}^{\epsilon^{*}}$, tais que

$$
M_{\kappa, p, \Omega}^{\epsilon^{*}}=\bigcup_{j=1}^{k} \Upsilon_{j}
$$

e $h_{j} \in C\left([0,1] \times \Upsilon_{j}, M_{\kappa, p, \Omega}^{\epsilon^{*}}\right)$ tais que

$$
h_{j}(0, u)=u, h_{j}(1, u)=u_{j} \in M_{\kappa, p, \Omega}^{\epsilon^{*}}, \text { para cada } u \in \Upsilon_{j}, j=1, \ldots, k,
$$


e $u_{j} \in M_{\kappa, p, \Omega}^{\epsilon^{*}}$ fixos.

Considere os conjuntos $B_{j}:=\Phi_{\kappa, p}^{-1}\left(\Upsilon_{j}\right), j=1, \ldots, k$, os quais são fechados em $\Omega_{r}^{-}$e

$$
\Omega_{r}^{-}=\bigcup_{j=1}^{k} B_{j} .
$$

Pela Proposição 2.12, as aplicações $g_{j}:[0,1] \times B_{j} \rightarrow \Omega_{r}^{+}, j=1, \ldots, k$, dadas por

$$
g_{j}(t, y):=\beta\left(h_{j}\left(t, \Phi_{\kappa, p}(y)\right)\right)
$$

estão bem definidas. Mais ainda, $g_{j} \in C\left([0,1] \times B_{j}, \Omega_{r}^{+}\right)$e

$$
g_{j}(0, y)=y, g_{j}(1, y)=y_{j} \in \Omega_{r}^{+} \text {, para cada } y \in B_{j}, j=1, \ldots, k,
$$

e $y_{j} \in \Omega_{r}^{+}$fixos. Portanto, como $\Omega_{r}^{+}$e $\Omega_{r}^{-}$são homotopicamente equivalentes a $\Omega$,

$$
\operatorname{cat}(\Omega)=\operatorname{cat}_{\Omega_{r}^{+}}\left(\Omega_{r}^{-}\right) \leq k,
$$

como queríamos.

\section{Demonstração do Teorema 2.1.}

Para $\kappa \geq 0$ fixado, tomemos $\bar{p}=\bar{p}(\kappa) \in\left(2,2^{*}\right)$ dado na Proposição 2.13, $p \in\left[\bar{p}, 2^{*}\right), \epsilon(\kappa)>0$ dado na Proposição 2.12 e $\epsilon^{*}=\epsilon^{*}(\kappa)>0$ dado em (2.30). Se $\epsilon^{*}=\frac{1}{N} S^{\frac{N}{2}}+\rho$ é um valor crítico de $I_{\kappa, p, \Omega}$ para todo $\rho \in(0, \epsilon(\kappa))$, então $I_{\kappa, p, \Omega}$ possui infinitos pontos críticos e a prova está completa. Por outro lado, admitamos que $\epsilon^{*}$ é um valor regular de $I_{\kappa, p, \Omega}$. Como $M_{\kappa, p, \Omega}^{\epsilon^{*}}$ é um conjunto fechado de $M_{\kappa, p, \Omega}$, tendo em vista as Proposição 2.6, $I_{\kappa, p, \Omega}$ restrito a $M_{\kappa, p, \Omega}^{\epsilon^{*}}$ satisfaz a condição $(P S)_{d}$, para todo $d \in \mathbb{R}$. Logo podemos aplicar a teoria de Ljusternik-Schnirelmann e a Proposição 2.14 para obtemos cat $(\Omega)$ pontos críticos de $\left.I_{\kappa, p, \Omega}\right|_{M_{\kappa, p, \Omega}^{*}}$. Pelo fato de $\epsilon^{*}$ ser valor regular, obtemos cat $(\Omega)$ pontos críticos de $\left.I_{\kappa, p, \Omega}\right|_{M_{\kappa, p, \Omega}}$. Pelo Corolário 2.7, estes são pontos críticos de $I_{\kappa, p, \Omega}$.

\subsection{Provas dos Teorema 2.2 e Corolário 2.3}

Nesta seção usaremos os resultados mostrados na seção A.3 do Apêndice A. Verifiquemos, então, que as condições exigidas na referida são satisfeitas. De fato, para $\kappa \geq 0$ fixado:

- a condição $(i)$ segue de como o funcional $I_{\kappa, p, \Omega}$ foi definido;

- $($ ii $)$ segue da Proposição 2.4 e de $H_{I_{\kappa, p, \Omega}}(u): E_{A} \times E_{A} \rightarrow \mathbb{R}$ dada por

$$
H_{I_{\kappa, p, \Omega}}(u)(v, w)=\langle v, w\rangle_{E_{A}}-(p-1) \int_{\Omega}|u|^{p-2} \operatorname{Re}(w \bar{v}) \mathrm{d} x, \quad \forall v, w \in E_{A},
$$

ser uma forma bilinear simétrica, para todo $u \in E_{A}$; 
- $($ iii) e (iv) são consequências da Proposição 2.5;

- $(v)$ segue pelo Corolário 2.13 para $p \in\left[\bar{p}, 2^{*}\right): \operatorname{temos} \Phi_{\kappa, p}: \Omega_{r}^{-} \rightarrow M_{\kappa, p, \Omega}^{\epsilon^{*}} \mathrm{e}$ $\beta: M_{\kappa, p, \Omega}^{\epsilon^{*}} \rightarrow \Omega_{r}^{+}$, onde $\epsilon^{*}$, valor regular de $I_{\kappa, p, \Omega}$, é dado em (2.30), e, por construção $\beta \circ \Phi_{\kappa, p}=I d_{\Omega_{r}^{-}}, \Omega_{r}^{+}, \Omega_{r}^{+}$e $\Omega$ homotopicamente equivalentes.

Assim, tomando, para $p \in\left[\bar{p}, 2^{*}\right)$, os entes matemáticos deste capítulo que correspondem aos que foram fixados na Seção A.3 do Apêndice A, vamos às demonstrações dos resultados principais.

\section{Demonstração do Teorema 2.2.}

Seja, para $\kappa \geq 0$ fixado, $p \in\left[\bar{p}, 2^{*}\right), \bar{p}=\bar{p}(\kappa)$ dado no Lema 2.13. Seja também $\mathcal{K}$ o conjunto dos pontos críticos de $I_{\kappa, p, \Omega}$. Suponha que $\mathcal{K}$ é discreto.

Definamos

$$
\mathcal{C}_{1}:=\left\{u \in \mathcal{K} ; \delta<I_{\kappa, p, \Omega}(u) \leq \epsilon^{*}\right\}
$$

$\mathrm{e}$

$$
\mathcal{C}_{2}:=\left\{u \in \mathcal{K} ; \epsilon^{*}<I_{\kappa, p, \Omega}(u)\right\},
$$

$\operatorname{com} \delta \in\left(0, \delta_{p}\right)$ dado na Proposição $2.5, \epsilon^{*}=\epsilon^{*}(\kappa)$ como em (2.30). Então, como $\mathcal{C}_{1}, \mathcal{C}_{2}$ são conjuntos críticos isolados e $\mathcal{K}=\mathcal{C}_{1} \cup \mathcal{C}_{2}$, pelas propriedades do índice de Morse generalizado

$$
i_{t}\left(\mathcal{K}, I_{\kappa, p, \Omega}\right)=i_{t}\left(\mathcal{C}_{1},\left.I_{\kappa, p, \Omega}\right|_{\overline{\Sigma\left(\delta, \epsilon^{*}\right)}}\right)+i_{t}\left(\mathcal{C}_{2},\left.I_{\kappa, p, \Omega}\right|_{\overline{\Sigma\left(\epsilon^{*},+\infty\right)}}\right)=\sum_{u \in \mathcal{C}_{1}} i_{t}(u)+\sum_{u \in \mathcal{C}_{2}} i_{t}(u) .
$$

Pelo Lema A.26,

$$
\sum_{u \in \mathcal{C}_{1}} i_{t}(u)=t \mathcal{P}_{t}(\Omega)+t \mathcal{Q}(t)+(1+t) \mathcal{Q}_{1}(t)
$$

e

$$
\sum_{u \in \mathcal{C}_{2}} i_{t}(u)=t^{2}\left[\mathcal{P}_{t}(\Omega)+\mathcal{Q}(t)-1\right]+(1+t) \mathcal{Q}_{2}(t)
$$

Então, como $\mathcal{Q}$ é um polinômio com coeficientes não negativos,

$$
\sum_{u \in \mathcal{K}} i_{t}(u)=t \mathcal{P}_{t}(\Omega)+t^{2}\left[\mathcal{P}_{t}(\Omega)-1\right]+(1+t) \mathcal{Q}_{3}(t),
$$

onde $\mathcal{Q}_{3}$ é um polinômio com coeficientes não negativos.

\section{Demonstração do Corolário 2.3.}

Seja, para $\kappa \geq 0$ fixado, $p \in\left[\bar{p}, 2^{*}\right), \bar{p}=\bar{p}(\kappa)$ dado no Lema 2.13. Seja também $\mathcal{K}$ o conjunto dos pontos críticos de $I_{\kappa, p, \Omega}$. Suponha que todos os pontos críticos de $I_{\kappa, p, \Omega}$ são não degenerados. Basta, então, observar que, nestas circunstâncias,

$$
i(u)=t^{m(u)}, \text { para todo } u \in \mathcal{K} .
$$





\section{Multiplicidade de soluções não-triviais para equações de Schrödinger não-lineares com presença de campo magnético externo e condição de fronteira mista: caso homogêneo}

Este capítulo é dedicado ao estudo do problema de fronteira mista para a equação de Schrödinger com campo magnético

$$
\left\{\begin{aligned}
\left(-i \nabla-A_{\lambda}\right)^{2} u+u & =|u|^{q-2} u, \text { em } \Omega_{\lambda} \\
u & =0, \text { sobre } \Gamma_{0 \lambda} \\
\frac{\partial u}{\partial \nu} & =0, \text { sobre } \Gamma_{1 \lambda}
\end{aligned}\right.
$$

onde $i$ é a unidade imaginária, $\lambda$ é um parâmetro positivo, $q \in\left(2,2^{*}\right), \Omega_{\lambda}:=\lambda \Omega$ um domínio em expansão, $\Omega \subset \mathbb{R}^{N}, N \geq 3$, é um domínio limitado, com fronteira suave $\partial \Omega=\bar{\Gamma}_{0} \cup \bar{\Gamma}_{1}$, 
onde $\Gamma_{0}, \Gamma_{1}$ são subvariedades suaves disjuntas, a medida de Lebesgue $(N-1)$-dimensional de $\Gamma_{1}$ é positiva em $\mathbb{R}^{N}, \Gamma_{0 \lambda}:=\lambda \Gamma_{0}, \Gamma_{1 \lambda}:=\lambda \Gamma_{1}, A \in C(\Omega, \mathbb{R}), A_{\lambda}():.=A(\dot{\bar{\lambda}})$.

Para preservarmos a uniformidade da notação neste capítulo e no próximo, denotaremos a não linearidade da seguinte forma:

$\left(f_{1}\right) f: \mathbb{R}_{+} \rightarrow \mathbb{R}_{+}$é uma função homogênea de grau $\frac{q-2}{2}, \operatorname{com} q \in\left(2,2^{*}\right)$,

a qual tem a função $s \mapsto|s|^{q-2} s$ como caso particular. De $\left(f_{1}\right)$, temos:

$\left(f_{2}\right) f(s)=f(1) s^{\frac{q-2}{2}}$, para todo $s \geq 0$

$\left(f_{3}\right) F(s):=\int_{0}^{s} f(t) \mathrm{d} t$ é $\frac{q}{2}$-homogênea para $s \geq 0$ e

$$
\frac{1}{2} F(s)=\frac{1}{q} s f(s)=\frac{f(1)}{q} s^{\frac{q}{2}}, \forall s \geq 0
$$

$\left(f_{4}\right)$ para cada $s>0$,

$$
f^{\prime}(s)=f(1)\left(\frac{q-2}{2}\right) s^{\frac{q-4}{2}}>0
$$

Os principais resultados deste capítulo são os seguintes:

Teorema 3.1. Existe $\lambda^{*}>0$ tal que, para $\lambda>\lambda^{*}$, o problema $\left(P_{A_{\lambda}}\right)$ possui, pelo menos, $\operatorname{cat}\left(\Gamma_{1 \lambda}\right)$ soluções.

Teorema 3.2. Seja $\lambda^{*}>0$ dado no Teorema 3.1. Se $\lambda>\lambda^{*}$ e o conjunto $\mathcal{K}$ de soluções de $\left(P_{A_{\lambda}}\right)$ é discreto, então

$$
\sum_{u \in \mathcal{K}} i_{t}(u)=t \mathcal{P}_{t}\left(\Gamma_{1 \lambda}\right)+t^{2}\left[\mathcal{P}_{t}\left(\Gamma_{1 \lambda}\right)-1\right]+(t+1) \mathcal{Q}(t)
$$

onde $\mathcal{Q}$ é um polinômio de coeficientes inteiros não-negativos, $\mathcal{P}_{t}\left(\Gamma_{1 \lambda}\right)$ é o polinômio de Poincaré de $\Gamma_{1 \lambda}$ e $i_{t}(u)$ é o indice de Morse generalizado de $u$.

No caso em que as soluções de $\left(P_{A_{\lambda}}\right)$ são não-degeneradas, temos o

Corolário 3.3. Seja $\lambda^{*}>0$ dado no Teorema 3.1. Se $\lambda>\lambda^{*}$ e as soluções de $\left(P_{A_{\lambda}}\right)$ são não-degeneradas, então

$$
\sum_{u \in \mathcal{K}} t^{m(u)}=t \mathcal{P}_{t}\left(\Gamma_{1 \lambda}\right)+t^{2}\left[\mathcal{P}_{t}\left(\Gamma_{1 \lambda}\right)-1\right]+(t+1) \mathcal{Q}(t)
$$

onde $\mathcal{Q}$ é um polinômio de coeficientes inteiros não-negativos, $\mathcal{P}_{t}\left(\Gamma_{1 \lambda}\right)$ é o polinômio de Poincaré de $\Gamma_{1 \lambda}$ e $m_{t}(u)$ é o indice de Morse numérico de $u$. 


\subsection{Condição (PS)}

Para cada $\lambda>0$, definamos o espaço de Hilbert sobre o corpo dos reais

$$
H_{A_{\lambda}}^{1}\left(\Omega_{\lambda}, \Gamma_{0 \lambda}\right):=\left\{u \in L^{2}\left(\Omega_{\lambda}, \mathbb{C}\right) ;\left|\nabla_{A_{\lambda}} u\right| \in L^{2}\left(\Omega_{\lambda}\right), \operatorname{traço}(u)=0 \text { em } \Gamma_{0 \lambda}\right\},
$$

onde

$$
\nabla_{A_{\lambda}} u:=\left(D_{A_{\lambda}}^{j} u\right)_{j=1}^{N}, \quad D_{A_{\lambda}}^{j} u:=-i \partial_{j} u-A_{\lambda}^{j} u, \quad j \in\{1, \ldots, N\},
$$

munido do produto interno

$$
<u, v>_{A_{\lambda}}:=\operatorname{Re}\left\{\int_{\Omega_{\lambda}}\left(\nabla_{A_{\lambda}} u \overline{\nabla_{A_{\lambda}}}+u \bar{v}\right) \mathrm{d} x\right\},
$$

onde $\operatorname{Re}(w)$ é a parte real de $w \in \mathbb{C}$ e $\bar{w}$ é o seu conjugado complexo. A norma induzida por este produto interno é dada por

$$
\|u\|_{A_{\lambda}}:=\left(\int_{\Omega_{\lambda}}\left(\left|\nabla_{A_{\lambda}} u\right|^{2}+|u|^{2}\right) \mathrm{d} x\right)^{1 / 2} .
$$

A seguir enunciaremos uma versão da desigualdade diamagnética presente em [26].

Lema 3.4. Vale

$$
\left|\nabla_{A_{\lambda}} u\right| \geq|\nabla| u||, \quad \forall u \in H_{A_{\lambda}}^{1}\left(\Omega_{\lambda}, \Gamma_{0 \lambda}\right) .
$$

Mais ainda, a imersão $H_{A_{\lambda}}^{1}\left(\Omega_{\lambda}, \Gamma_{0 \lambda}\right) \hookrightarrow L^{p}\left(\Omega_{\lambda}, \mathbb{R}\right)$ é compacta para cada $p \in\left[1,2^{*}\right)$ e é continua para cada $p \in\left[1,2^{*}\right]$, com constantes de imersão independentes de $\lambda>0$, pela suavidade do conjunto $\Omega$ (ver [2]).

Vale ressaltar que, além do resultado acima, a suavidade do domínio $\Omega$ também nos dá a seguinte propriedade: existe $r_{0}>0$ tal que, para todo $y \in \partial \Omega$,

$$
B_{y}^{+}:=B_{r_{0}}\left(y+r_{0} \nu_{y}\right) \subset \Omega \quad \text { e } \quad B_{y}^{-}:=B_{r_{0}}\left(y-r_{0} \nu_{y}\right) \subset \mathbb{R}^{N} \backslash \Omega .
$$

Diremos que $u \in H_{A_{\lambda}}^{1}\left(\Omega_{\lambda}, \Gamma_{0 \lambda}\right)$ é uma solução fraca de $P_{A_{\lambda}}$ se

$$
\operatorname{Re}\left\{\int_{\Omega_{\lambda}}\left(\mid \nabla_{A_{\lambda}} u \overline{\nabla_{A_{\lambda}} u}+u \bar{v}\right) \mathrm{d} x-\int_{\Omega_{\lambda}} f\left(|u|^{2}\right) u \bar{v} \mathrm{~d} x\right\}=0, \quad \forall v \in H_{A_{\lambda}}^{1}\left(\Omega_{\lambda}, \Gamma_{0 \lambda}\right) .
$$

Para cada $\lambda>0$, o funcional energia associado ao problema $\left(P_{A_{\lambda}}\right) I_{\lambda}: H_{A_{\lambda}}^{1}\left(\Omega_{\lambda}, \Gamma_{0 \lambda}\right)$ é dado por

$$
I_{\lambda}(u):=\frac{1}{2} \int_{\Omega_{\lambda}}\left(\left|\nabla_{A_{\lambda}} u\right|^{2}+|u|^{2}\right) \mathrm{d} x-\frac{1}{2} \int_{\Omega_{\lambda}} F\left(|u|^{2}\right) \mathrm{d} x,
$$

Por $\left(f_{1}\right), I_{\lambda}$ está bem definido e é de classe $C^{2}\left(H_{A_{\lambda}}^{1}\left(\Omega_{\lambda}, \Gamma_{0 \lambda}\right), \mathbb{R}\right)$. Além disso

$$
I_{\lambda}^{\prime}(u) v:=\operatorname{Re}\left\{\int_{\Omega_{\lambda}}\left(\nabla_{A_{\lambda}} u \overline{\nabla_{A_{\lambda}} v}+u \bar{v}\right) \mathrm{d} x-\int_{\Omega_{\lambda}} f\left(|u|^{2}\right) u \bar{v} \mathrm{~d} x\right\}
$$

para todo $u, v \in H_{A_{\lambda}}^{1}\left(\Omega_{\lambda}, \Gamma_{0 \lambda}\right)$. Assim, todo ponto crítico de $I_{\lambda}$ é uma solução fraca de $\left(P_{A_{\lambda}}\right)$. 
56 Capítulo 3 - Equações de Schrödinger com condição de fronteira mista: caso homogêneo

Proposição 3.5. O funcional $I_{\lambda}$ satisfaz a condição de Palais-Smale, isto é, toda sequência $\left(u_{n}\right) \subset H_{A_{\lambda}}^{1}\left(\Omega_{\lambda}, \Gamma_{0 \lambda}\right)$ com $\sup _{n \in \mathbb{N}}\left|I_{\lambda}\left(u_{n}\right)\right|<+\infty$ e $I_{\lambda}^{\prime}\left(u_{n}\right) \rightarrow 0$ quando $n \rightarrow \infty$, possui uma subsequência convergente.

\section{Demonstração.}

Dada uma sequência $\left(u_{n}\right) \subset H_{A_{\lambda}}^{1}\left(\Omega_{\lambda}, \Gamma_{0 \lambda}\right)$ tal que $\sup _{n \in \mathbb{N}}\left|I_{\lambda}\left(u_{n}\right)\right|<+\infty$ e $I_{\lambda}^{\prime}\left(u_{n}\right) \rightarrow 0$ quando $n \rightarrow \infty$, podemos supor que, para algum $d \in \mathbb{R}, I_{\lambda}\left(u_{n}\right) \rightarrow d$ e $I_{\lambda}^{\prime}\left(u_{n}\right) \rightarrow 0$ quando $n \rightarrow \infty$.

Observemos que $\left(u_{n}\right)$ é limitada. Com efeito, usando $\left(f_{3}\right)$,

$$
\begin{aligned}
d+o_{n}(1)+o_{n}(1)\left\|u_{n}\right\|_{A_{\lambda}} & =I_{\lambda}\left(u_{n}\right)-\frac{1}{q} I_{\lambda}^{\prime}\left(u_{n}\right) u_{n} \\
& =\left(\frac{1}{2}-\frac{1}{q}\right)\left\|u_{n}\right\|_{A_{\lambda}}^{2}+\int_{\Omega_{\lambda}}\left(\frac{1}{q} f\left(\left|u_{n}\right|^{2}\right)\left|u_{n}\right|^{2}-\frac{1}{2} F\left(\left|u_{n}\right|^{2}\right)\right) \mathrm{d} x \\
& =\left(\frac{1}{2}-\frac{1}{q}\right)\left\|u_{n}\right\|_{A_{\lambda}}^{2},
\end{aligned}
$$

donde segue a limitação de $\left(u_{n}\right)$. Consequentemente, existe $u \in H_{A_{\lambda}}^{1}\left(\Omega_{\lambda}, \Gamma_{0 \lambda}\right)$ tal que, a menos de subsequência,

$$
\left\{\begin{array}{l}
u_{n} \rightarrow u \text { em } H_{A_{\lambda}}^{1}\left(\Omega_{\lambda}, \Gamma_{0 \lambda}\right), \\
u_{n} \rightarrow u \operatorname{em~} L^{p}\left(\Omega_{\lambda}, \mathbb{C}\right), \forall p \in\left[1,2^{*}\right), \\
u_{n}(x) \rightarrow u(x) \text { quase sempre em } \Omega_{\lambda},
\end{array}\right.
$$

quando $n \rightarrow \infty$. Notemos que, pela definição de $I_{\lambda}^{\prime}$,

$$
\left\|u_{n}-u\right\|_{A_{\lambda}}^{2}=\left(I_{\lambda}^{\prime}\left(u_{n}\right)-I_{\lambda}^{\prime}(u)\right)\left(u_{n}-u\right)-\operatorname{Re}\left\{\int_{\Omega_{\lambda}}\left(f\left(\left|u_{n}\right|^{2}\right) u_{n}-f\left(|u|^{2}\right) u\right) \overline{\left(u_{n}-u\right)} \mathrm{d} x\right\} .
$$

Dessa forma, usando $\left(f_{2}\right),(3.2)$ e o fato de $\left.\left.\sup _{n}|| u_{n}\right|^{q-2} u_{n}\right|_{\frac{q}{q-1}, \Omega_{\lambda}}<\infty$, obtemos

$$
\begin{aligned}
\left\|u_{n}-u\right\|_{A_{\lambda}}^{2} \leq & \left|I_{\lambda}^{\prime}\left(u_{n}\right)\left(u_{n}-u\right)\right|+\left|I_{\lambda}^{\prime}(u)\left(u_{n}-u\right)\right|+ \\
& +\int_{\Omega_{\lambda}}\left|f\left(\left|u_{n}\right|^{2}\right) u_{n}-f\left(|u|^{2}\right) u\right|\left|u_{n}-u\right| \mathrm{d} x \\
= & \left|I_{\lambda}^{\prime}\left(u_{n}\right)\left(u_{n}-u\right)\right|+\left|I_{\lambda}^{\prime}(u)\left(u_{n}-u\right)\right|+ \\
& +\left.\int_{\Omega_{\lambda}} f(1)|| u_{n}\right|^{q-2} u_{n}-|u|^{q-2} u|| u_{n}-u \mid \mathrm{d} x \\
= & o_{n}(1),
\end{aligned}
$$

quando $n \rightarrow \infty$. Portanto, $u_{n} \rightarrow u$ em $H_{A_{\lambda}}^{1}\left(\Omega_{\lambda}, \Gamma_{0 \lambda}\right)$ a menos de subsequência.

Tendo em vista a homogeneidade de $f$, o funcional $I_{\lambda}$ possui a geometria do passo da montanha. Dessa forma, pela Proposição 3.5 e pelo Teorema do Passo da Montanha de Ambrosetti-Rabinowitz, para cada $\lambda>0,\left(P_{A_{\lambda}}\right)$ possui pelo menos uma solução não-trivial 
$u_{\lambda} \in H_{A_{\lambda}}^{1}\left(\Omega_{\lambda}, \Gamma_{0 \lambda}\right)$, tal que $I_{\lambda}\left(u_{\lambda}\right)=b_{\lambda}$ e $I_{\lambda}^{\prime}\left(u_{\lambda}\right)=0$, onde $b_{\lambda}$ denota o nível do passo da montanha de $I_{\lambda}$. Usando mais uma vez a homogeneidade de $f$, argumentando como em [43, Theorem 4.2], temos

$$
b_{\lambda}:=\inf _{u \in M_{\lambda}} I_{\lambda}(u)
$$

com

$$
M_{\lambda}=\left\{u \in H_{A_{\lambda}}^{1} \backslash\{0\} ; I_{\lambda}^{\prime}(u) u=0\right\},
$$

a variedade Nehari de $I_{\lambda}$.

Sobre o funcional $I_{\lambda}$ e a variedade $M_{\lambda}$ temos os seguintes resultados.

Proposição 3.6. A variedade $M_{\lambda}$ é difeomorfa à esfera unitária em $H_{A_{\lambda}}^{1}\left(\Omega_{\lambda}, \Gamma_{0 \lambda}\right)$. Além disso, existe $\delta_{0}>0$ independente de $\lambda>0$ tal que, para todo $u \in M_{\lambda}$,

$$
\|u\|_{A_{\lambda}} \geq \delta_{0} \quad \text { e } \quad I_{\lambda}(u) \geq \delta_{0} .
$$

\section{Demonstração.}

A demonstração da primeira parte segue os passos da demonstração da Proposição 1.7. Seja $u \in M_{\lambda}$ qualquer. Pela homogeneidade de $f$, temos

$$
\|u\|_{A_{\lambda}}^{2}=\int_{\Omega_{\lambda}} f\left(|u|^{2}\right)|u|^{2}=f(1)|u|_{\Omega_{\lambda}, q}^{q} \leq C_{q}\|u\|_{A_{\lambda}}^{q},
$$

onde $C_{q}$ é a constante de imersão $H_{A_{\lambda}}^{1}\left(\Omega_{\lambda}, \Gamma_{0 \lambda}\right) \hookrightarrow L^{q}\left(\Omega_{\lambda}, \mathbb{C}\right)$, a qual, pelo Lema 3.4, independe de $\lambda>0$. Portanto,

$$
\|u\|_{A_{\lambda}} \geq\left(\frac{1}{C_{q}}\right)^{\frac{1}{q-2}}=: \delta_{1}>0 .
$$

Notemos que, por $\left(f_{3}\right)$, como $u \in M_{\lambda}$,

$$
\frac{1}{q}\|u\|_{A_{\lambda}}^{2}=\frac{1}{q} \int_{\Omega_{\lambda}} f\left(|u|^{2}\right)|u|^{2}=\frac{1}{2} \int_{\Omega_{\lambda}} F\left(|u|^{2}\right) .
$$

Assim, por (3.4) e (3.5),

$$
I_{\lambda}(u)=\frac{1}{2}\|u\|_{A_{\lambda}}^{2}-\frac{1}{2} \int_{\Omega_{\lambda}} F\left(|u|^{2}\right)=\left(\frac{1}{2}-\frac{1}{q}\right)\|u\|_{A_{\lambda}}^{2} \geq\left(\frac{1}{2}-\frac{1}{q}\right) \delta_{1}=: \delta_{2} .
$$

Por fim, basta tomar $\delta_{0}:=\min \left\{\delta_{1}, \delta_{2}\right\}$.

Proposição 3.7. O funcional $\left.I_{\lambda}\right|_{M_{\lambda}}$ satisfaz a condição de Palais-Smale.

\section{Demonstração.}

Seja $\left(u_{n}\right) \in M_{\lambda}$ uma sequência tal que $\sup _{n \in \mathbb{N}}\left|I_{\lambda}\left(u_{n}\right)\right|<+\infty$ e $\left(\left.I_{\lambda}\right|_{M_{\lambda}}\right)^{\prime}\left(u_{n}\right) \rightarrow 0$ quando $n \rightarrow \infty$. A menos de subsequência, podemos supor que, para algum $d \in \mathbb{R}, I_{\lambda}\left(u_{n}\right) \rightarrow d$ quando $n \rightarrow \infty$. Como em [43, Proposição 5.12], existe $\mu_{n} \in \mathbb{R}$, para todo $n \in \mathbb{N}$, tal que

$$
I_{\lambda}^{\prime}\left(u_{n}\right)-\mu_{n} G_{\lambda}^{\prime}\left(u_{n}\right)=\left(\left.I_{\lambda}\right|_{M_{\lambda}}\right)^{\prime}\left(u_{n}\right)=o_{n}(1), \quad \forall n \in \mathbb{N},
$$


onde

$$
G_{\lambda}(v)=I_{\lambda}^{\prime}(v) v, \quad \forall v \in H_{A_{\lambda}}^{1}\left(\Omega_{\lambda}, \Gamma_{0 \lambda}\right) .
$$

Como na Proposição 3.5, $\left(u_{n}\right)$ é limitada e existe $u \in H_{A_{\lambda}}^{1}\left(\Omega_{\lambda}, \Gamma_{0 \lambda}\right)$ tal que, a menos de subsequência,

$$
\left\{\begin{array}{l}
u_{n} \rightarrow u \text { em } H_{A_{\lambda}}^{1}\left(\Omega_{\lambda}, \Gamma_{0 \lambda}\right), \\
u_{n} \rightarrow u \text { em } L^{p}\left(\Omega_{\lambda}, \mathbb{C}\right), \forall p \in\left[1,2^{*}\right), \\
u_{n} \rightarrow u \text { quase sempre em } \Omega_{\lambda},
\end{array}\right.
$$

quando $n \rightarrow \infty$. Observe que, como $u_{n} \in M_{\lambda}$,

$$
\begin{aligned}
G_{\lambda}^{\prime}\left(u_{n}\right) u_{n} & =2 \int_{\Omega_{\lambda}}\left|\nabla_{A_{\lambda}} u_{n}\right|^{2}+\left|u_{n}\right|^{2}-f\left(\left|u_{n}\right|^{2}\right)\left|u_{n}\right|^{2}-f^{\prime}\left(\left|u_{n}\right|^{2}\right)\left|u_{n}\right|^{4} \\
& =-2 \int_{\Omega_{\lambda}} f^{\prime}\left(\left|u_{n}\right|^{2}\right)\left|u_{n}\right|^{4} \\
& =-f(1)(q-2) \int_{\Omega_{\lambda}}\left|u_{n}\right|^{q} .
\end{aligned}
$$

Além disso, pela Proposição 3.6, existe $\delta_{0}>0$ tal que

$$
\left\|u_{n}\right\|_{A_{\lambda}} \geq \delta_{0}, \quad \forall n \in \mathbb{N}
$$

ou seja, como $u_{n} \in M_{\lambda}$,

$$
f(1) \int_{\Omega_{\lambda}}\left|u_{n}\right|^{q}=\int_{\Omega_{\lambda}} f\left(\left|u_{n}\right|^{2}\right)\left|u_{n}\right|^{2} \geq \delta_{0}, \quad \forall n \in \mathbb{N} .
$$

Aplicando $u_{n}$ a ambos os membros de (3.6) e usando (3.7) e (3.8), obtemos

$$
\mu_{n}=o_{n}(1)
$$

ou seja, $\left(u_{n}\right)$ também satisfaz $I_{\lambda}^{\prime}\left(u_{n}\right) \rightarrow 0$ quando $n \rightarrow \infty$. O resultado segue da Proposição 3.5 .

Procedendo como na prova da Proposição 3.7, podemos mostrar o próximo resultado.

Corolário 3.8. Todo ponto crítico de $\left.I_{\lambda}\right|_{M_{\lambda}}$ é um ponto crítico de $I_{\lambda}$.

\subsection{Preliminares}

Nesta seção, nosso objetivo é buscar relações entre subconjuntos de $\mathbb{R}^{N}$ e do espaço de funções $H_{A_{\lambda}}^{1}\left(\Omega_{\lambda}, \Gamma_{0 \lambda}\right)$. Sejam os problemas

$$
\left\{\begin{aligned}
-\Delta u+u & =f\left(u^{2}\right) u, \text { em } \mathbb{R}_{+}^{N} \\
\frac{\partial u}{\partial \nu} & =0, \text { sobre } \mathbb{R}^{N-1}
\end{aligned}\right.
$$


onde $\mathbb{R}^{N-1}=\left\{\left(x_{1}, \ldots, x_{N-1}, 0\right) ; x_{i} \in \mathbb{R}, i=1, \ldots, N-1\right\}$, e

$$
\left\{\begin{array}{l}
-\Delta u+u=f\left(u^{2}\right) u, \text { em } \mathbb{R}^{N}, \\
u \in H^{1}\left(\mathbb{R}^{N}\right), u>0 \text { em } \mathbb{R}^{N},
\end{array}\right.
$$

e consideremos seus respectivos funcionais energia associados

$$
J_{\infty}(u):=\frac{1}{2} \int_{\mathbb{R}_{+}^{N}}\left(|\nabla u|^{2}+u^{2}\right) \mathrm{d} x-\frac{1}{2} \int_{\mathbb{R}_{+}^{N}} F\left(u^{2}\right) \mathrm{d} x, \quad \forall u \in H^{1}\left(\mathbb{R}_{+}^{N}, \mathbb{R}\right),
$$

$\mathrm{e}$

$$
J_{\mathbb{R}^{N}}(u):=\frac{1}{2} \int_{\mathbb{R}^{N}}\left(|\nabla u|^{2}+u^{2}\right) \mathrm{d} x-\frac{1}{2} \int_{\mathbb{R}^{N}} F\left(u^{2}\right) \mathrm{d} x, \quad \forall u \in H^{1}\left(\mathbb{R}^{N}, \mathbb{R}\right) .
$$

Consideremos também as variedades de Nehari e níveis do passo da montanha correspondentes:

$$
N_{\infty}:=\left\{u \in H^{1}\left(\mathbb{R}_{+}^{N}, \mathbb{R}\right) \backslash\{0\} ; J_{\infty}^{\prime}(u) u=0\right\}, \quad c_{\infty}:=\inf _{N_{\infty}} J_{\infty}
$$

e

$$
N_{\mathbb{R}^{N}}:=\left\{u \in H^{1}\left(\mathbb{R}^{N}, \mathbb{R}\right) \backslash\{0\} ; J_{\mathbb{R}^{N}}^{\prime}(u) u=0\right\}, \quad c_{\mathbb{R}^{N}}:=\inf _{N_{\mathbb{R}^{N}}} J_{\mathbb{R}^{N}} .
$$

Por [12, 35], $\left(P_{2}\right)$ possui uma solução positiva e radialmente simétrica $w \in H^{1}\left(\mathbb{R}^{N}, \mathbb{R}\right) \cap C^{2}\left(\mathbb{R}^{N}\right)$. Além disso, a restrição de $w$ a $\mathbb{R}_{+}^{N}$ é solução de $\left(P_{1}\right)$. Como consequência,

$$
c_{\mathbb{R}^{N}}=2 c_{\infty}
$$

Seja $r>0$ tal que

$$
\Gamma_{1}^{+}:=\left\{x \in \mathbb{R}^{N} ; \operatorname{dist}\left(x, \Gamma_{1}\right) \leq r\right\}, \quad \Gamma_{1}^{-}:=\left\{x \in \Gamma_{1} ; \operatorname{dist}\left(x, \Gamma_{0}\right) \geq r\right\}
$$

são homotopicamente equivalentes a $\Gamma_{1}$ (ver Apêndice A, Definição A.3). Seja $\eta \in C^{\infty}\left(\mathbb{R}_{+}\right)$ uma função não crescente tal que $\eta=1 \mathrm{em}[0, r / 2], \eta=0 \mathrm{em}[r,+\infty), \eta^{\prime} \in L^{\infty}\left(\mathbb{R}_{+}\right)$. Denotaremos por $\Gamma_{1 \lambda}^{-}$o conjunto $\lambda \Gamma_{1}^{-}$. Para qualquer $y \in \Gamma_{1 \lambda}^{-}$, definimos a função

$$
x \in \Omega_{\lambda} \mapsto e^{i \tau_{\lambda, y}(x)} \eta\left(\frac{|x-y|}{\lambda}\right) w(x-y),
$$

onde $\tau_{\lambda, y}(x):=\sum_{j=1}^{N} A_{\lambda}^{j}(y) x^{j}$. Pela definição de $\eta$, esta função pertence a $H_{A_{\lambda}}^{1}\left(\Omega_{\lambda}, \Gamma_{0 \lambda}\right)$. Pela homogeneidade de $f$, existe $t_{\lambda, y}>0$ tal que

$$
t_{\lambda, y} e^{i \tau_{\lambda, y} \eta}\left(\frac{|.-y|}{\lambda}\right) w(.-y) \in M_{\lambda}
$$

Definamos, portanto, $\Phi_{\lambda}: \Gamma_{1 \lambda}^{-} \rightarrow M_{\lambda}$ como

$$
\Phi_{\lambda}(y)(x)=t_{\lambda, y} e^{i \tau_{\lambda, y}(x)} \eta\left(\frac{|x-y|}{\lambda}\right) w(x-y), \quad \forall x \in \Omega_{\lambda}, y \in \Gamma_{1 \lambda}^{-} .
$$


Lema 3.9. Sob a hipótese $\left(f_{1}\right)$, temos o limite

$$
\lim _{\lambda \rightarrow \infty} \max _{y \in \Gamma_{1 \lambda}^{-}}\left|I_{\lambda}\left(\Phi_{\lambda}(y)\right)-c_{\infty}\right|=0
$$

\section{Demonstração.}

Primeiramente notemos que, pela definição de $\nabla_{A_{\lambda}}$, para $y \in \Gamma_{1 \lambda}^{-}$qualquer,

$$
\left|\nabla_{A_{\lambda}} \Phi_{\lambda}(y)\right|^{2}=\sum_{j=1}^{N}\left|D_{A_{\lambda}}^{j}\left(\Phi_{\lambda}(y)\right)\right|^{2},
$$

e para $x \in \Omega_{\lambda}$,

$$
\begin{aligned}
D_{A_{\lambda}}^{j}\left(\Phi_{\lambda}(y)(x)\right)= & -i \partial_{j} \Phi_{\lambda}(y)(x)-A^{j}\left(\frac{x}{\lambda}\right) \Phi_{\lambda}(y)(x) \\
= & t_{\lambda, y} e^{i \tau_{\lambda, y}(x)}\left[-A^{j}\left(\frac{x}{\lambda}\right) \eta\left(\frac{|x-y|}{\lambda}\right) w(x-y)-\right. \\
& -i \partial_{j}\left(\eta\left(\frac{|x-y|}{\lambda}\right) w(x-y)\right)+ \\
& \left.+\eta\left(\frac{|x-y|}{\lambda}\right) w(x-y) A^{j}\left(\frac{y}{\lambda}\right)\right] \\
= & t_{\lambda, y} e^{i \tau_{\lambda, y(x)}}\left[\eta\left(\frac{|x-y|}{\lambda}\right) w(x-y)\left(A^{j}\left(\frac{y}{\lambda}\right)-A^{j}\left(\frac{x}{\lambda}\right)\right)-\right. \\
& \left.-i \partial_{j}\left(\eta\left(\frac{|x-y|}{\lambda}\right) w(x-y)\right)\right]
\end{aligned}
$$

Portanto

$$
\begin{aligned}
\left|\nabla_{A_{\lambda}} \Phi_{\lambda}(y)\right|^{2}= & t_{\lambda, y}^{2}\left[\eta^{2}\left(\frac{|x-y|}{\lambda}\right) w^{2}(x-y)\left|A\left(\frac{y}{\lambda}\right)-A\left(\frac{x}{\lambda}\right)\right|^{2}+\right. \\
& \left.+\left|\nabla\left(\eta\left(\frac{|x-y|}{\lambda}\right) w(x-y)\right)\right|^{2}\right]
\end{aligned}
$$

Temos, então, para $y \in \Gamma_{1 \lambda}^{-}$qualquer,

$$
\begin{aligned}
I_{\lambda}\left(\Phi_{\lambda}(y)\right)= & \frac{1}{2} \int_{\Omega_{\lambda}}\left[\left|\nabla_{A_{\lambda}}\left(\Phi_{\lambda}(y)\right)\right|^{2}+\left|\Phi_{\lambda}(y)\right|^{2}-F\left(\left|\Phi_{\lambda}(y)\right|^{2}\right)\right] \mathrm{d} x \\
= & \frac{t_{\lambda, y}^{2}}{2} \int_{\Omega_{\lambda}}\left\{\eta^{2}\left(\frac{|x-y|}{\lambda}\right) w^{2}(x-y)\left|A\left(\frac{y}{\lambda}\right)-A\left(\frac{x}{\lambda}\right)\right|^{2}+\right. \\
& \left.+\left|\nabla\left(\eta\left(\frac{|x-y|}{\lambda}\right) w(x-y)\right)\right|^{2}+\eta^{2}\left(\frac{|x-y|}{\lambda}\right) w^{2}(x-y)\right\} \mathrm{d} x- \\
& -\int_{\Omega_{\lambda}} F\left(t_{\lambda, y}^{2}\left|\eta\left(\frac{|x-y|}{\lambda}\right) w(x-y)\right|^{2}\right) \mathrm{d} x
\end{aligned}
$$

Seja $T_{y}$ a matriz de rotação em $\mathbb{R}^{N}$ tal que o vetor normal unitário interior a $T_{y}\left(\Omega_{\lambda}-y\right)$ seja $e_{N}:=(0,0, \ldots, 0,1) \in \mathbb{R}^{N}$, e façamos $\tilde{\Omega}_{\lambda, y}:=T_{y}\left(\Omega_{\lambda}-y\right)$. Então, fazendo uma mudança de 
variáveis, lembrando que $\eta$ e $w$ são radialmente simétricas e $T_{y}$ é uma matriz de rotação, temos

$$
\begin{aligned}
I_{\lambda}\left(\Phi_{\lambda}(y)\right)= & \frac{t_{\lambda, y}^{2}}{2} \int_{\tilde{\Omega}_{\lambda, y}} \eta^{2}\left(\frac{|z|}{\lambda}\right)\left[w^{2}(z)+|(\nabla w)(z)|^{2}\right] \mathrm{d} z- \\
& -\frac{1}{2} \int_{\tilde{\Omega}_{\lambda, y}} F\left(t_{\lambda, y}^{2} \eta^{2}\left(\frac{|z|}{\lambda}\right) w^{2}(z)\right) \mathrm{d} z+ \\
& +\frac{t_{\lambda, y}^{2}}{2} \int_{\tilde{\Omega}_{\lambda, y}}\left|A\left(\frac{y}{\lambda}\right)-A\left(\frac{T_{y}^{-1} z+y}{\lambda}\right)\right|^{2} \eta^{2}\left(\frac{|z|}{\lambda}\right) w^{2}(z) \mathrm{d} z+ \\
& +\frac{t_{\lambda, y}^{2}}{2} \int_{\tilde{\Omega}_{\lambda, y}} \frac{1}{\lambda^{2}}\left|\eta^{\prime}\left(\frac{|z|}{\lambda}\right)\right|^{2} w^{2}(z) \mathrm{d} z+ \\
& +\frac{t_{\lambda, y}^{2}}{2} \int_{\tilde{\Omega}_{\lambda, y}} \frac{2}{\lambda}\left|\eta^{\prime}\left(\frac{|z|}{\lambda}\right)\right| w(z) \eta\left(\frac{|z|}{\lambda}\right)|\nabla w(z)| \mathrm{d} z
\end{aligned}
$$

Afirmamos que as integrais em (3.10), (3.11) e (3.12) tendem a zero quando $\lambda \rightarrow+\infty$.

Sobre (3.10), observemos que, como $w \in L^{2}\left(\mathbb{R}^{N}\right)$, dado $\epsilon>0$, existe $M>0$ tal que

$$
\int_{\tilde{\Omega}_{\lambda, y} \cap B_{M}^{c}(0)}\left|A\left(\frac{y}{\lambda}\right)-A\left(\frac{T_{y}^{-1} z+y}{\lambda}\right)\right|^{2} \eta^{2}\left(\frac{|z|}{\lambda}\right) w^{2}(z) \mathrm{d} z<\epsilon .
$$

Para concluirmos o limite para (3.10), resta, portanto, mostrar que

$$
\lim _{\lambda \rightarrow \infty} \int_{\tilde{\Omega}_{\lambda, y} \cap B_{M}(0)}\left|A\left(\frac{y}{\lambda}\right)-A\left(\frac{T_{y}^{-1} z+y}{\lambda}\right)\right|^{2} \eta^{2}\left(\frac{|z|}{\lambda}\right) w^{2}(z) \mathrm{d} z=0 .
$$

Como $A$ é uniformemente contínua no compacto $\bar{\Omega}$, para $\epsilon>0$ dado anteriormente, existe $\gamma>0$ tal que

$$
|A(x+v)-A(x)|<\epsilon, \quad \forall|v| \leq \gamma, \quad \forall x \in \bar{\Omega} .
$$

Como $T_{y}$ é uma matriz de rotação, $\left|T_{y}^{-1} z\right| \leq M$, para todo $z \in \tilde{\Omega}_{\lambda, y} \cap B_{M}(0)$. Assim, para $\lambda>0$ suficientemente grande, temos $\left|\frac{T_{y}^{-1} z}{\lambda}\right| \leq \gamma$ e, por (3.14), para $\lambda$ suficientemente grande,

$$
\left|A\left(\frac{y+T_{y}^{-1} z}{\lambda}\right)-A\left(\frac{y}{\lambda}\right)\right|<\epsilon, \quad \forall y \in \Gamma_{1 \lambda}^{-} .
$$

Dessa forma, para $\lambda$ suficientemente grande,

$$
\chi_{\tilde{\Omega}_{\lambda, y} \cap B_{M}(0)}(z) \eta^{2}\left(\frac{|z|}{\lambda \rho}\right) w^{2}(z)\left|A\left(\frac{y+T_{y}^{-1} z}{\lambda}\right)-A\left(\frac{y}{\lambda}\right)\right|^{2} \leq \epsilon^{2}|w|_{\infty, \mathbb{R}^{N}}^{2}, \quad \forall z \in B_{M}(0),
$$

e, por conseguinte, vale (3.13).

Com relação a (3.11) e (3.12), notemos que, para uma constante $C>0$ adequada,

$$
\begin{aligned}
& \chi_{\tilde{\Omega}_{\lambda, y}} \frac{1}{\lambda}\left[\frac{1}{2 \lambda}\left|\eta^{\prime}\left(\frac{|\cdot|}{\lambda}\right)\right|^{2} w^{2}+\left|\eta^{\prime}\left(\frac{|\cdot|}{\lambda}\right)\right|\right.\left.\eta\left(\frac{|\cdot|}{\lambda}\right) w|\nabla w|\right] \leq \\
& \leq C\left[w^{2}+w|\nabla w|\right] \in L^{1}\left(\mathbb{R}^{N}\right)
\end{aligned}
$$


$\mathrm{e}$

$$
\begin{aligned}
\chi_{\tilde{\Omega}_{\lambda, y}}(z) \frac{1}{\lambda}\left[\frac{1}{2 \lambda}\left|\eta^{\prime}\left(\frac{|z|}{\lambda}\right)\right|^{2}\right. & \left.w^{2}(z)+\left|\eta^{\prime}\left(\frac{|z|}{\lambda}\right)\right| \eta\left(\frac{|z|}{\lambda}\right) w(z)|\nabla w(z)|\right] \leq \\
& \leq \frac{c}{\lambda}\left[\frac{1}{\lambda}\left|\eta^{\prime}\right|_{\infty, \mathbb{R}_{+}}^{2}|w|_{\infty, \mathbb{R}^{N}}^{2}+\left|\eta^{\prime}\right|_{\infty, \mathbb{R}_{+}}|w|_{\infty, \mathbb{R}^{N}}|\nabla w|_{\infty, \mathbb{R}^{N}}\right] \stackrel{\lambda \rightarrow+\infty}{\rightarrow} 0
\end{aligned}
$$

Segue, portanto, do Teorema da Convergência Dominada de Lebesgue que (3.11) e (3.12) tendem a zero quando $\lambda \rightarrow+\infty$.

No que segue, usaremos o mesmo argumento empregado no Lema 1.13 .Fixemos, então, $\lambda_{n} \rightarrow \infty$ quando $n \rightarrow \infty, y_{n} \in \Omega_{\lambda_{n}}$, para todo $n \in \mathbb{N}$, quaisquer e mostremos que

$$
\lim _{n \rightarrow \infty} I_{\lambda_{n}}\left(\Phi_{\lambda_{n}}\left(y_{n}\right)\right)=c_{\infty}
$$

Retomando o raciocínio, temos, depois de mostrarmos que as integrais em (3.10), (3.11) e (3.12) tendem a zero quando $\lambda \rightarrow+\infty$, fazendo $\Omega_{n}:=\Omega_{\lambda_{n}}, \tilde{\Omega}_{n}:=\tilde{\Omega}_{\lambda_{n}, y_{n}}, t_{n}:=t_{\lambda_{n}, y_{n}}$,

$$
\begin{aligned}
I_{\lambda_{n}}\left(\Phi_{\lambda_{n}}\left(y_{n}\right)\right)= & \frac{t_{n}^{2}}{2} \int_{\tilde{\Omega}_{n}} \eta^{2}\left(\frac{|z|}{\lambda_{n}}\right)\left[|(\nabla w)(z)|^{2}+w^{2}(z)\right] \mathrm{d} z- \\
& -\frac{1}{2} \int_{\tilde{\Omega}_{n}} F\left(t_{n}^{2} \eta^{2}\left(\frac{|z|}{\lambda_{n}}\right) w^{2}(z)\right) \mathrm{d} z+o_{n}(1) t_{n}
\end{aligned}
$$

Mostremos que $t_{n} \rightarrow 1$.

Combinando a definição de $t_{n}$ com o estudo feito nas integrais (3.10)-(3.12), temos

$$
\begin{aligned}
& o_{n}(1)+\int_{\tilde{\Omega}_{n}} \eta^{2}\left(\frac{|z|}{\lambda_{n}}\right) {\left[|\nabla w|^{2}+w^{2}\right] \mathrm{d} z=} \\
&=\int_{\tilde{\Omega}_{n}} f\left(t_{n}^{2} \eta^{2}\left(\frac{|z|}{\lambda_{n}}\right) w^{2}\right) \eta^{2}\left(\frac{|z|}{\lambda_{n}}\right) w^{2} \mathrm{~d} z .
\end{aligned}
$$

Notemos que $\left(t_{n}\right)$ é limitada. De fato, suponhamos por contradição que $t_{n} \rightarrow+\infty$, a menos de subsequência. Observemos que a homogeneidade de $f$ implica

$$
\lim _{t \rightarrow \infty} f\left(t^{2}\right)=+\infty
$$

Pelo Teorema da Convergência Dominada de Lebesgue, (3.16), (3.1), a definição de $\eta$, Lema de Fatou e o limite (3.17), temos

$$
\begin{aligned}
+\infty & >\int_{\mathbb{R}^{N}}\left(|\nabla w|^{2}+w^{2}\right) \mathrm{d} z \\
& =\lim _{n \rightarrow \infty} \int_{\tilde{\Omega}_{n}} \eta^{2}\left(\frac{|z|}{\lambda_{n}}\right)\left[|\nabla w(z)|^{2}+w^{2}(z)\right] \mathrm{d} z \\
& \geq \lim _{n \rightarrow \infty} \int_{B_{r_{0}}\left(r_{0} e_{N}\right)} f\left(t_{n}^{2} \eta^{2}\left(\frac{|z|}{\lambda_{n}}\right) w^{2}(z)\right) \eta^{2}\left(\frac{|z|}{\lambda_{n}}\right) w^{2}(z) \mathrm{d} z \\
& =\lim _{n \rightarrow \infty} \int_{B_{r_{0}}\left(r_{0} e_{N}\right)} f\left(t_{n}^{2} w^{2}(z)\right) w^{2}(z) \mathrm{d} z \\
& =+\infty
\end{aligned}
$$


o que é um absurdo. Portanto $\left(t_{n}\right)$ é limitada. Admitamos, sem perder a generalidade, que $t_{n} \rightarrow t_{0}$ quando $n \rightarrow \infty$. Verifiquemos que $t_{0}>0$. Com efeito, suponhamos por contradição que $t_{0}=0$. Pelo Teorema da Convergência Dominada de Lebesgue, usando a homogeneidade de $f$,

$$
\lim _{n \rightarrow \infty} \int_{\tilde{\Omega}_{n}} f\left(t_{n}^{2} \eta^{2}\left(\frac{|z|}{\lambda_{n}}\right) w^{2}(z)\right) \eta^{2}\left(\frac{|z|}{\lambda_{n}}\right) w^{2}(z) \mathrm{d} z=0 .
$$

Portanto, por (3.16), (3.1) e o limite (3.18), temos

$$
\begin{aligned}
0 & <\int_{\mathbb{R}_{+}^{N}}\left(|\nabla w|^{2}+w^{2}\right) \mathrm{d} z \\
& =\lim _{n \rightarrow \infty} \int_{\tilde{\Omega}_{n}} \eta^{2}\left(\frac{|z|}{\lambda_{n}}\right)\left(|\nabla w(z)|^{2}+w^{2}(z)\right) \mathrm{d} z \\
& =\lim _{n \rightarrow \infty} \int_{\tilde{\Omega}_{n}} f\left(t_{n}^{2} \eta^{2}\left(\frac{|z|}{\lambda_{n}}\right) w^{2}(z)\right) \eta^{2}\left(\frac{|z|}{\lambda_{n}}\right) w^{2}(z) \mathrm{d} z \\
& =0
\end{aligned}
$$

que é um absurdo. Logo $t_{n} \rightarrow t_{0}>0$ quando $n \rightarrow \infty$. Agora observemos que

$$
\begin{aligned}
\int_{\mathbb{R}^{N}}\left(|\nabla w|^{2}+w^{2}\right) \mathrm{d} z & =\lim _{n \rightarrow \infty} \int_{\tilde{\Omega}_{n}} t_{n}^{2} \eta^{2}\left(\frac{|z|}{\lambda_{n}}\right)\left[|\nabla w(z)|^{2}+w^{2}(z)\right] \mathrm{d} z \\
& =\lim _{n \rightarrow \infty} \int_{\tilde{\Omega}_{n}} f\left(t_{n}^{2} \eta^{2}\left(\frac{|z|}{\lambda_{n}}\right) w^{2}(z)\right) \eta^{2}\left(\frac{|z|}{\lambda_{n}}\right) w^{2}(z) \mathrm{d} z \\
& =\int_{\mathbb{R}^{N}} f\left(t_{0}^{2} w^{2}\right) w^{2} \mathrm{~d} z
\end{aligned}
$$

Pelas propriedades de $w$ e $\left(f_{4}\right), t_{0}=1$. Assim, o resultado segue por (3.15) e do Teorema da Convergência Dominada de Lebesgue.

Vejamos uma versão para o nosso problema do conhecido Lema de Lions (ver [43]), o qual é fundamental para os nossos estudos.

Lema 3.10. Sejam $l>0$ e $2 \leq p<2^{*}$. Se a família $\left\{u_{\lambda} \in H^{1}\left(\Omega_{\lambda}, \mathbb{R}\right)\right\}_{\lambda>0}$ é limitada uniformemente em $\lambda>0$ e satisfaz

$$
\lim _{\lambda \rightarrow \infty} \sup _{y \in \mathbb{R}^{N}} \int_{B_{l}(y) \cap \Omega_{\lambda}}\left|u_{\lambda}\right|^{p} \mathrm{~d} x=0,
$$

então

$$
\lim _{\lambda \rightarrow \infty} \int_{\Omega_{\lambda}}\left|u_{\lambda}\right|^{m} \mathrm{~d} x=0, \quad \forall m \in\left(2,2^{*}\right) .
$$

Demonstração.

Sejam $\lambda>0$ e $p<m<2^{*}$ quaisquer.

Note que existe $\alpha \in(0,1)$ tal que

$$
\frac{1}{m}=\frac{\alpha}{2^{*}}+\frac{1-\alpha}{p} .
$$


Pela desigualdade de interpolação e das imersões de Sobolev,

$$
\begin{aligned}
\left|u_{\lambda}\right|_{m, B_{l}(y) \cap \Omega_{\lambda}} & \leq\left|u_{\lambda}\right|_{p, B_{l}(y) \cap \Omega_{\lambda}}^{1-\alpha}\left|u_{\lambda}\right|_{2^{*}, B_{l}(y) \cap \Omega_{\lambda}}^{\alpha} \\
& \leq C_{2^{*}}\left|u_{\lambda}\right|_{p, B_{l}(y) \cap \Omega_{\lambda}}^{1-\alpha}\left\|u_{\lambda}\right\|_{B_{l}(y) \cap \Omega_{\lambda}}^{\alpha} .
\end{aligned}
$$

Tomando $\alpha=\frac{2}{m}$, isto é, para $m=p+2-\frac{2 p}{2^{*}}$, temos

$$
\int_{B_{l}(y) \cap \Omega_{\lambda}}\left|u_{\lambda}\right|^{m} \mathrm{~d} x \leq C_{2^{*}}^{m}|u|_{p, B_{l}(y) \cap \Omega_{\lambda}}^{(1-\alpha) m}\|u\|_{B_{l}(y) \cap \Omega_{\lambda}}^{2} .
$$

Cobrindo $\mathbb{R}^{N}$ com bolas de raio $l$ de modo que cada ponto do espaço esteja contido em, no máximo, $\Lambda=\Lambda(N)$ bolas, temos

$$
\int_{\Omega_{\lambda}}\left|u_{\lambda}\right|^{m} \mathrm{~d} x \leq \Lambda C_{2^{*}}^{m} \sup _{y \in \mathbb{R}^{N}}\left[\int_{B_{l}(y) \cap \Omega_{\lambda}}|u|^{p} \mathrm{~d} x\right]^{(1-\alpha) \frac{m}{p}}\|u\|_{\Omega_{\lambda}}^{2} .
$$

Portanto, usando a hipótese, e lembrando que, a partir do Lema $3.4, C_{2^{*}}$ não depende de $\lambda$

$$
\lim _{\lambda \rightarrow \infty}\left|u_{\lambda}\right|_{m, \Omega_{\lambda}}=0
$$

Aplicando novamente pela desigualdade de interpolação e as imersões de Sobolev,

$$
\lim _{\lambda \rightarrow \infty}\left|u_{\lambda}\right|_{m, \Omega_{\lambda}}=0, \quad \forall m \in\left(2,2^{*}\right) .
$$

\subsection{Comportamento dos níveis minimax}

Sobre o nível $b_{\lambda}$ definido em (3.3), temos

Proposição 3.11. $\lim _{\lambda \rightarrow \infty} b_{\lambda}=c_{\infty}$.

A demonstração da Proposição 3.11 é longa. Por isso a dividiremos em alguns lemas.

Primeiramente observemos que pela definição de $\Phi_{\lambda}(y)$ e o Lema 3.9,

$$
b_{\lambda} \leq I_{\lambda}\left(\Phi_{\lambda}(y)\right) \leq o_{\lambda}(1)+c_{\infty} .
$$

Consideremos os problemas auxiliares

$$
\left\{\begin{aligned}
-\Delta u+u & =f\left(u^{2}\right) u, \text { em } \Omega_{\lambda} \\
\frac{\partial u}{\partial \nu} & =0, \text { sobre } \Gamma_{1 \lambda} \\
u & =0, \text { sobre } \Gamma_{0 \lambda}
\end{aligned}\right.
$$

e

$$
\left\{\begin{aligned}
-\Delta u+u & =f\left(u^{2}\right) u, \text { em } \Omega_{\lambda} \\
\frac{\partial u}{\partial \nu} & =0, \text { sobre } \partial \Omega_{\lambda} .
\end{aligned}\right.
$$


Denotaremos por $H^{1}\left(\Omega_{\lambda}, \Gamma_{0 \lambda}\right)$ o espaço de Hilbert

$$
H^{1}\left(\Omega_{\lambda}, \Gamma_{0 \lambda}\right):=\left\{u \in H^{1}\left(\Omega_{\lambda}, \mathbb{R}\right) ; \operatorname{traço}(u)=0 \text { em } \Gamma_{0 \lambda}\right\}
$$

munido da norma usual de $H^{1}\left(\Omega_{\lambda}, \mathbb{R}\right)$, a qual denotaremos por $\|\cdot\|_{\Omega_{\lambda}}$. Sejam $J_{\lambda}: H^{1}\left(\Omega_{\lambda}, \Gamma_{0 \lambda}\right) \rightarrow \mathbb{R}$, o funcional energia associado ao problema $\left(P_{3}\right)$, dado por

$$
J_{\lambda}(u)=\frac{1}{2} \int_{\Omega_{\lambda}}\left(|\nabla u|^{2}+u^{2}\right) \mathrm{d} x-\frac{1}{2} \int_{\Omega_{\lambda}} F\left(u^{2}\right) \mathrm{d} x, \quad \forall u \in H^{1}\left(\Omega_{\lambda}, \Gamma_{0 \lambda}\right) .
$$

e $c_{\lambda}$ o nível do passo da montanha associado. Definimos também $\bar{J}_{\lambda}: H^{1}\left(\Omega_{\lambda}, \mathbb{R}\right) \rightarrow \mathbb{R}$ o o funcional energia associado ao problema $\left(P_{4}\right)$, por

$$
\bar{J}_{\lambda}(u)=\frac{1}{2} \int_{\Omega_{\lambda}}\left(|\nabla u|^{2}+u^{2}\right) \mathrm{d} x-\frac{1}{2} \int_{\Omega_{\lambda}} F\left(u^{2}\right) \mathrm{d} x, \quad \forall u \in H^{1}\left(\Omega_{\lambda}, \mathbb{R}\right)
$$

com variedade de Nehari e nível do passo da montanha correspondentes

$$
\bar{N}_{\lambda}:=\left\{u \in H^{1}\left(\Omega_{\lambda}, \mathbb{R}\right) \backslash\{0\} ; \bar{J}^{\prime}(u) u=0\right\} \quad \text { e } \quad \bar{c}_{\lambda}:=\inf _{\bar{N}_{\lambda}} \bar{J}_{\lambda} .
$$

Pela definição dos níveis dados anteriormente e pela desigualdade diamagnética (ver Lema $3.4)$, temos

$$
b_{\lambda} \geq c_{\lambda} \geq \bar{c}_{\lambda}>0
$$

Por (3.19) e (3.20), é suficiente mostrarmos que

$$
\lim _{\lambda \rightarrow \infty} \bar{c}_{\lambda}=c_{\infty}
$$

Para provarmos o limite (3.21), comecemos observando que, argumentando como na

Proposição 3.5 e usando o Teorema do Passo da Montanha, existe $u_{\lambda} \in H^{1}\left(\Omega_{\lambda}, \mathbb{R}\right)$ tal que

$$
\bar{J}_{\lambda}\left(u_{\lambda}\right)=\bar{c}_{\lambda}=\inf _{\bar{N}_{\lambda}} \bar{J}_{\lambda}, \quad \bar{J}_{\lambda}^{\prime}\left(u_{\lambda}\right)=0,
$$

para cada $\lambda>0$. Por $(3.20)$ e $(3.22),\left(\bar{J}_{\lambda}\left(u_{\lambda}\right)\right)_{\lambda}$ é limitada e $\bar{J}_{\lambda}^{\prime}\left(u_{\lambda}\right) u_{\lambda}=0$, para todo $\lambda>0$. Daí, por $\left(f_{3}\right)$,

$$
\sup _{\lambda>0}\left\|u_{\lambda}\right\|_{\Omega_{\lambda}}<+\infty .
$$

Procedendo como na demonstração da Proposição 3.6, podemos supor, sem perda de generalidade,

$$
\left\|u_{\lambda}\right\|_{\Omega_{\lambda}}^{2} \geq \delta_{0} \quad \text { e } \quad \bar{J}_{\lambda}\left(u_{\lambda}\right) \geq \bar{c}_{\lambda} \geq \delta_{0}, \quad \forall \lambda>0,
$$

onde, como antes, $\delta_{0}>0$ independe de $\lambda$. Portanto, por (3.24) e pelo Lema 3.10, existem $\left(y_{\lambda}\right)_{\lambda} \subset \mathbb{R}^{N}, l>0$ e $\gamma>0$ tais que

$$
\liminf _{\lambda \rightarrow \infty} \int_{\Omega_{\lambda} \cap B_{l}\left(y_{\lambda}\right)}\left|u_{\lambda}\right|^{2} \geq \gamma>0
$$

Mais ainda, aumentando $l$ se necessário, podemos supor que $y_{\lambda} \in \Omega_{\lambda}$, para todo $\lambda>0$, uma vez que (3.25) implica que $\Omega_{\lambda} \cap B_{l}\left(y_{\lambda}\right) \neq 0$, para todo $\lambda$. 
66 Capítulo 3 - Equações de Schrödinger com condição de fronteira mista: caso homogêneo

Lema 3.12. Existe uma constante $C>0$ tal que $\operatorname{dist}\left(y_{\lambda}, \partial \Omega_{\lambda}\right) \leq C$.

\section{Demonstração.}

Suponha, por contradição, que existe $\lambda_{n} \rightarrow+\infty$ tal que $\operatorname{dist}\left(y_{\lambda_{n}}, \partial \Omega_{\lambda_{n}}\right) \rightarrow \infty$, quando $n \rightarrow \infty$. Seja $R>l$ arbitrário. Para $n$ suficientemente grande, $B_{2 R}\left(y_{\lambda_{n}}\right) \subset \Omega_{\lambda_{n}}$. Definamos

$$
w_{\lambda_{n}, R}(x):=\eta\left(\frac{|x|}{R}\right) u_{\lambda_{n}}\left(x+y_{\lambda_{n}}\right), \quad \forall x \in \Omega_{\lambda_{n}}-y_{\lambda_{n}},
$$

onde $\eta \in C^{\infty}\left(\mathbb{R}_{+}\right)$é tal que $\eta=1 \mathrm{em}[0,1], \eta=0$ em $(2,+\infty), \eta^{\prime} \in L^{\infty}\left(\mathbb{R}_{+}\right)$. Então $\operatorname{supp} w_{\lambda_{n}, R} \subset B_{2 R}(0)$. Sem perda de generalidade, podemos supor que $w_{\lambda_{n}, R} \in H^{1}\left(\mathbb{R}^{N}, \mathbb{R}\right)$, estendendo esta aplicação por zero fora de $\Omega_{\lambda_{n}}-y_{\lambda_{n}}$. Mais ainda, por construção, $\sup _{n}\left\|w_{\lambda_{n}, R}\right\| \leq C$, para alguma constante $C>0$ independente de $R$. Além disso, como

$$
\int_{B_{l}(0)}\left|w_{\lambda_{n}, R}\right|^{2} \mathrm{~d} x=\int_{B_{l}(0)}\left|u_{\lambda_{n}}\left(x+y_{\lambda_{n}}\right)\right|^{2} \mathrm{~d} x=\int_{B_{l}\left(y_{\lambda_{n}}\right)}\left|u_{\lambda_{n}}\right|^{2} \mathrm{~d} x \geq \gamma>0
$$

existe $w_{R} \in H^{1}\left(\mathbb{R}^{N}, \mathbb{R}\right) \backslash\{0\}$ tal que

$$
\left\{\begin{array}{l}
w_{\lambda, R} \rightarrow w_{R} \text { em } H^{1}\left(\mathbb{R}^{N}, \mathbb{R}\right), \text { quando } n \rightarrow \infty, \\
w_{\lambda, R} \rightarrow w_{R}, \text { em } L_{l o c}^{p}\left(\mathbb{R}^{N}, \mathbb{R}\right), p \in\left[1,2^{*}\right), \text { quando } n \rightarrow \infty \\
\int_{B_{l}(0)}\left|w_{R}\right|^{2} \mathrm{~d} x \geq \gamma>0 .
\end{array}\right.
$$

Já que $\left\|w_{R}\right\| \leq \liminf _{n \rightarrow \infty}\left\|w_{\lambda_{n}, R}\right\|$, a família $\left(w_{R}\right)_{R} \subset H^{1}\left(\mathbb{R}^{N}, \mathbb{R}\right)$ é limitada. Assim, existe $v \in H^{1}\left(\mathbb{R}^{N}, \mathbb{R}\right)$ tal que

$$
\left\{\begin{array}{l}
w_{R} \rightarrow v \text { em } H^{1}\left(\mathbb{R}^{N}, \mathbb{R}\right), \text { quando } R \rightarrow \infty \\
w_{R} \rightarrow v, \text { em } L_{l o c}^{p}\left(\mathbb{R}^{N}, \mathbb{R}\right), p \in\left[1,2^{*}\right), \text { quando } R \rightarrow \infty \\
\int_{B_{l}(0)}|v|^{2} \mathrm{~d} x \geq \gamma>0
\end{array}\right.
$$

Em particular, $v \not \equiv 0$. Mostremos que $v$ é uma solução de $\left(P_{2}\right)$. De fato, dada $\phi \in C_{c}^{\infty}\left(\mathbb{R}^{N}\right)$, tomemos $t>0$ tal que supp $\phi \subset B_{t}(0)$. Para $n$ suficientemente grande, $B_{t}\left(y_{\lambda_{n}}\right) \subset \Omega$. Como $u_{\lambda_{n}}$ é solução de $\left(P_{4}\right)$ para $\lambda_{n}=\lambda$, temos

$$
\begin{aligned}
\int_{B_{t}(0)}\left[\nabla u_{\lambda_{n}}\left(x+y_{\lambda_{n}}\right) \nabla \phi+u_{\lambda_{n}}\left(x+y_{\lambda_{n}}\right) \phi\right] & =\int_{\Omega_{\lambda_{n}}}\left[\nabla u_{\lambda_{n}} \nabla \phi\left(x-y_{\lambda_{n}}\right)+u_{\lambda_{n}} \phi\left(x-y_{\lambda_{n}}\right)\right] \\
& =\int_{\Omega_{\lambda_{n}}} f\left(u_{\lambda_{n}}^{2}\right) u_{\lambda_{n}} \phi\left(x-y_{\lambda_{n}}\right) \\
& =\int_{B_{t}(0)} f\left(u_{\lambda_{n}}^{2}\left(x+y_{\lambda_{n}}\right)\right) u_{\lambda_{n}}\left(x+y_{\lambda_{n}}\right) \phi .
\end{aligned}
$$

Para $n$ suficientemente grande e $R>t$, obtemos

$$
\int_{B_{t}(0)}\left[\nabla w_{\lambda_{n}, R} \nabla \phi+w_{\lambda_{n}, R} \phi\right] \mathrm{d} x=\int_{B_{t}(0)} f\left(w_{\lambda_{n}, R}^{2}\right) w_{\lambda_{n}, R} \phi \mathrm{d} x .
$$


Passando ao limite $n \rightarrow \infty$, encontramos

$$
\int_{B_{t}(0)}\left[\nabla w_{R} \nabla \phi+w_{R} \phi\right] \mathrm{d} x=\int_{B_{t}(0)} f\left(w_{R}^{2}\right) w_{R} \phi \mathrm{d} x .
$$

Lembrando que supp $\phi \subset B_{t}(0)$ e $R>t$, tomando o limite $R \rightarrow \infty$, temos

$$
\int_{\mathbb{R}^{N}}[\nabla v \nabla \phi+v \phi] \mathrm{d} x=\int_{B_{t}(0)}[\nabla v \nabla \phi+v \phi] \mathrm{d} x=\int_{B_{t}(0)} f\left(v^{2}\right) v \phi \mathrm{d} x=\int_{\mathbb{R}^{N}} f\left(v^{2}\right) v \phi \mathrm{d} x .
$$

Como $\phi \in C_{c}^{\infty}\left(\mathbb{R}^{N}\right)$ é arbitrária, argumentando como em [43, Seção 1.6, Lema 1.30], $v$ é solução de $\left(P_{2}\right)$.

Notemos que, para $M \leq R$ dado, $B_{M}\left(y_{\lambda_{n}}\right) \subset \Omega_{\lambda_{n}}$. Por (3.19) e (3.20),

$$
\begin{aligned}
o_{\lambda}(1)+c_{\infty} & \geq \bar{c}_{\lambda_{n}}=\bar{J}_{\lambda_{n}}\left(u_{\lambda_{n}}\right)-\frac{1}{2} \bar{J}_{\lambda_{n}}^{\prime}\left(u_{\lambda_{n}}\right) u_{\lambda_{n}} \\
& =\frac{1}{2} \int_{\Omega_{\lambda_{n}}}\left[f\left(u_{\lambda_{n}}^{2}\right) u_{\lambda_{n}}^{2}-F\left(u_{\lambda_{n}}^{2}\right) u_{\lambda_{n}}^{2}\right] \mathrm{d} x \\
& \geq \frac{1}{2} \int_{B_{M}\left(y_{\lambda_{n}}\right)}\left[f\left(u_{\lambda_{n}}^{2}\right) u_{\lambda_{n}}^{2}-F\left(u_{\lambda_{n}}^{2}\right) u_{\lambda_{n}}^{2}\right] \mathrm{d} x \\
& =\frac{1}{2} \int_{B_{M}(0)}\left[f\left(w_{\lambda_{n}, r}^{2}\right) w_{\lambda_{n}, r}^{2}-F\left(w_{\lambda_{n}, r}^{2}\right) w_{\lambda_{n}, r}^{2}\right] \mathrm{d} x .
\end{aligned}
$$

Pelo Lema de Fatou e (3.9), obtemos, depois de tomar os limites $n \rightarrow \infty, R \rightarrow \infty$ e $M \rightarrow \infty$,

$$
c_{\infty} \geq \frac{1}{2} \int_{\mathbb{R}^{N}}\left[f\left(v^{2}\right) v^{2}-F\left(v^{2}\right)\right] \mathrm{d} x=J_{\mathbb{R}^{N}}(v) \geq c_{\mathbb{R}^{N}}=2 c_{\infty},
$$

o que é um absurdo.

Pelo Lema 3.12, aumentando $l$ se necessário, podemos admitir que $y_{\lambda} \in \Omega_{\lambda}$ em (3.25).

Seja $T_{y_{\lambda}}$ a matriz de rotação em $\mathbb{R}^{N}$ tal que o vetor normal unitário interior a $\tilde{\Omega}_{\lambda}:=T_{y_{\lambda}}\left(\Omega_{\lambda}-y_{\lambda}\right)$ seja $e_{N}=(0,0, \ldots, 0,1)$. Definamos, para todo $\lambda$,

$$
v_{\lambda}(x)=u_{\lambda}\left(T_{y_{\lambda}}^{-1} x+y_{\lambda}\right), \quad \forall x \in \tilde{\Omega}_{\lambda},
$$

a qual satisfaz:

(a) $\left\|v_{\lambda}\right\|_{\tilde{\Omega}_{\lambda}}=\left\|u_{\lambda}\right\|_{\Omega_{\lambda}}$, donde, por (3.23), $\sup _{\lambda>0}\left\|v_{\lambda}\right\|_{\tilde{\Omega}_{\lambda}}<+\infty$;

(b) $\int_{\tilde{\Omega}_{\lambda}} F\left(v_{\lambda}^{2}\right) \mathrm{d} x=\int_{\Omega_{\lambda}} F\left(u_{\lambda}^{2}\right) \mathrm{d} x ;$

(c) como $u_{\lambda}$ é solução de $\left(P_{4}\right), v_{\lambda}$ satisfaz o problema

$$
\left\{\begin{aligned}
-\Delta u+u & =f\left(u^{2}\right) u \text { em } \tilde{\Omega}_{\lambda} \\
\frac{\partial u}{\partial \nu} & =0 \text { em } \partial \tilde{\Omega}_{\lambda}
\end{aligned}\right.
$$


(d) $\bar{J}_{\tilde{\Omega}_{\lambda}}\left(v_{\lambda}\right)=\bar{c}_{\tilde{\Omega}_{\lambda}}=\bar{c}_{\lambda}$, onde $\bar{J}_{\tilde{\Omega}_{\lambda}}$ é o funcional energia associado ao problema $\left(P_{5}\right)$ e $\bar{c}_{\tilde{\Omega}_{\lambda}}$ o correspondente nível do passo da montanha;

(e) por (3.25),

$$
\liminf _{\lambda \rightarrow \infty} \int_{B_{l}(0) \cap \tilde{\Omega}_{\lambda}}\left|v_{\lambda}\right|^{2} \mathrm{~d} x \geq \gamma
$$

Dados $\rho>h>0$, definamos

$$
D_{\rho, h}:=\left\{x \in B_{\rho}(0) ; x^{N}>h, x=\left(x^{1}, \ldots, x^{N}\right)\right\} .
$$

Por (3.1), $\tilde{\Omega}_{\lambda} \rightarrow \mathbb{R}_{+}^{N}$ quando $\lambda \rightarrow \infty$, quase sempre em $\mathbb{R}^{N}$. Então, para $\lambda$ suficientemente grande, $D_{\rho, h} \subset \tilde{\Omega}_{\lambda}$. Logo $v_{\lambda} \in H^{1}\left(D_{\rho, h}, \mathbb{R}\right)$, para $\lambda$ suficientemente grande. Por $(a)$, existe $v_{\rho, h} \in H^{1}\left(D_{\rho, h}, \mathbb{R}\right)$ tal que, a menos de subsequência,

$$
\left\{\begin{array}{l}
v_{\lambda} \rightarrow v_{\rho, h} \text { em } H^{1}\left(D_{\rho, h}, \mathbb{R}\right), \text { quando } \lambda \rightarrow \infty \\
v_{\lambda} \rightarrow v_{\rho, h} \text { em } L^{p}\left(D_{\rho, h}, \mathbb{R}\right), p \in\left[1,2^{*}\right), \text { quando } \lambda \rightarrow \infty \\
v_{\lambda}(x) \rightarrow v_{\rho, h}(x) \text { quase sempre em } D_{\rho, h}, \text { quando } \lambda \rightarrow \infty .
\end{array}\right.
$$

Mais ainda, novamente por (a) e pelo Teorema de Banach-Steinhaus, existe uma constante $K>0$ tal que

$$
\left\|v_{\rho, h}\right\|_{D_{\rho, h}} \leq K, \quad \forall \rho, h>0 .
$$

Sejam $\rho_{n} \rightarrow \infty, h_{n} \rightarrow 0$ sequências monótonas. Então

$$
D_{n}:=D_{\rho_{n}, h_{n}} \subset D_{\rho_{n+1}, h_{n+1}}:=D_{n+1}, \quad \forall n \geq 1 .
$$

Observemos que: para $n=1$, existem $v_{1} \in H^{1}\left(D_{1}, \mathbb{R}\right)$ e subsequência $\left(v_{\lambda 1}\right) \subset\left(v_{\lambda}\right)$ tais que, quando $\lambda \rightarrow \infty$,

$$
\left\{\begin{array}{l}
v_{\lambda 1} \rightarrow v_{1} \text { em } H^{1}\left(D_{1}, \mathbb{R}\right) \\
v_{\lambda 1} \rightarrow v_{1} \text { em } L^{p}\left(D_{1}, \mathbb{R}\right), p \in\left[1,2^{*}\right) \\
v_{\lambda 1}(x) \rightarrow v_{1}(x) \text { quase sempre em } D_{1}
\end{array}\right.
$$

para $n=2$, existem $v_{2} \in H^{1}\left(D_{2}, \mathbb{R}\right)$ e subsequência $\left(v_{\lambda 2}\right) \subset\left(v_{\lambda 1}\right)$ tais que, quando $\lambda \rightarrow \infty$,

$$
\left\{\begin{array}{l}
v_{\lambda 2} \rightarrow v_{2} \text { em } H^{1}\left(D_{2}, \mathbb{R}\right) \\
v_{\lambda 2} \rightarrow v_{2} \text { em } L^{p}\left(D_{2}, \mathbb{R}\right), p \in\left[1,2^{*}\right) \\
v_{\lambda 2}(x) \rightarrow v_{2}(x) \text { quase sempre em } D_{2}
\end{array}\right.
$$

com $\left.v_{2}\right|_{D_{1}}=v_{1}$ quase sempre em $D_{1}$, pela unicidade do limite quase sempre em $D_{1}$; para $n=j$, existem $v_{j} \in H^{1}\left(D_{j}, \mathbb{R}\right)$ e subsequência $\left(v_{\lambda j}\right) \subset\left(v_{\lambda(j-1)}\right)$ tais que, quando $\lambda \rightarrow \infty$,

$$
\left\{\begin{array}{l}
v_{\lambda j} \rightarrow v_{j} \text { em } H^{1}\left(D_{j}, \mathbb{R}\right) \\
v_{\lambda j} \rightarrow v_{j} \text { em } L^{p}\left(D_{j}, \mathbb{R}\right), p \in\left[1,2^{*}\right) \\
v_{\lambda j}(x) \rightarrow v_{j}(x) \text { quase sempre em } D_{j}
\end{array}\right.
$$


com $\left.v_{j}\right|_{D_{l}}=v_{i}$ quase sempre em $D_{i}$, pela unicidade do limite quase sempre em $D_{i}$, para qualquer $i \leq j$. Seja $v_{k k} \in H^{1}\left(D_{k}, \mathbb{R}\right)$ o $k$-ésimo termo da subsequência $\left(v_{\lambda k}\right)$. Então $\left(v_{k k}\right)_{k},\left(\partial_{j} v_{k k}\right)_{k} \subset L^{2}\left(\mathbb{R}_{+}^{N}, \mathbb{R}\right)$ são sequências limitadas, donde, a menos de subsequência, convergem fraco em $L^{2}\left(\mathbb{R}_{+}^{N}, \mathbb{R}\right)$ para $v, \bar{v}_{j} \in L^{2}\left(\mathbb{R}_{+}^{N}, \mathbb{R}\right)$, respectivamente. Como $\partial_{j} v_{k k}$ é a $j$-ésima derivada parcial fraca de $v_{k k}$, temos, pela convergência fraca acima,

$$
\partial_{j} v=\bar{v}_{j}, \forall j \in\{1, \ldots, N\}, \text { quase sempre em } \mathbb{R}^{N} .
$$

Portanto, $v \in H^{1}\left(\mathbb{R}_{+}^{N}, \mathbb{R}\right)$ e, quando $k \rightarrow \infty$,

$$
\left\{\begin{array}{l}
v_{k k} \rightarrow v \text { em } H_{l o c}^{1}\left(\mathbb{R}_{+}^{N}, \mathbb{R}\right), \\
v_{k k} \rightarrow v \text { em } L_{l o c}^{p}\left(\mathbb{R}_{+}^{N}, \mathbb{R}\right), \forall p \in\left[1,2^{*}\right) \\
v_{k k}(x) \rightarrow v(x) \text { quase sempre em } \mathbb{R}_{+}^{N}
\end{array}\right.
$$

Para simplificar a notação, ponhamos $v_{k}:=v_{k k}$ e $\tilde{\Omega}_{k}:=\tilde{\Omega}_{k k}, \forall k \in \mathbb{N}$.

Lema 3.13. A função v é uma solução não trivial de $\left(P_{1}\right)$.

\section{Demonstração.}

Primeiramente vejamos que $v \not \equiv 0$. De fato, por $(e)$,

$$
\liminf _{k \rightarrow \infty} \int_{B_{l}(0) \cap \tilde{\Omega}_{k}} v_{k}^{2} \mathrm{~d} x \geq \gamma>0 .
$$

Dado $t \in(0, l)$, definamos $A_{t}:=\left\{x \in B_{l}(0) \cap \tilde{\Omega}_{k} ; 0 \leq x^{N} \leq t\right\}$ e $\Lambda_{k}:=\left(B_{l}(0) \cap \tilde{\Omega}_{k}\right) \backslash A_{t}$. Assim

$$
\int_{B_{l}(0) \cap \tilde{\Omega}_{k}} v_{k}^{2} \mathrm{~d} x=\left(\int_{A_{t}}+\int_{B_{k}}\right) v_{k}^{2} \mathrm{~d} x .
$$

Como $\sup _{k}\left\|v_{k}\right\|_{D_{k}}<\infty$, pela desigualdade de Hölder e das imersões de Sobolev, tendo em vista o Lema 3.4, existe uma constante $\bar{K}>0$ independente de $k$ tal que

$$
\int_{A_{t}} v_{k}^{2} \mathrm{~d} x \leq\left(\int_{A_{t}} v_{k}^{2^{*}} \mathrm{~d} x\right)^{\frac{2}{2^{*}}}\left(\int_{A_{t}} \mathrm{~d} x\right)^{\frac{2}{N}} \leq \bar{K}\left|A_{t}\right|^{\frac{2}{N}} .
$$

Escolhamos $t \in(0, l)$ de modo que

$$
\int_{A_{t}} v_{k}^{2} \mathrm{~d} x \leq\left(\int_{A_{t}} v_{k}^{2^{*}} \mathrm{~d} x\right)^{\frac{2}{2^{*}}}\left(\int_{A_{t}} \mathrm{~d} x\right)^{\frac{2}{N}} \leq \bar{K}\left|A_{t}\right|^{\frac{2}{N}}<\frac{\gamma}{4} .
$$

Assim, por (3.27), para todo $k$ suficientemente grande,

$$
\frac{\gamma}{2} \leq \int_{B_{l}(0) \cap \tilde{\Omega}_{k}} v_{k}^{2} \mathrm{~d} x \leq \frac{\gamma}{4}+\int_{\Lambda_{k}} v_{k}^{2} \mathrm{~d} x \leq \frac{\gamma}{4}+\int_{D} v_{k}^{2} \mathrm{~d} x
$$

para todo conjunto compacto $D \subset \mathbb{R}^{N}$ compacto com $\Lambda_{k} \subset D \subset D_{k}$. Logo, para todo $k$ suficientemente grande,

$$
\int_{D} v_{k}^{2} \mathrm{~d} x \geq \frac{\gamma}{4}
$$


e daí, por (3.26),

$$
\int_{D} v^{2} \mathrm{~d} x=\lim _{k \rightarrow \infty} \int_{D} v_{k}^{2} \mathrm{~d} x \geq \frac{\gamma}{4}>0
$$

Portanto $v \not \equiv 0$. Para mostrarmos que $v$ é solução fraca de $\left(P_{1}\right)$, vejamos que $\nabla v_{k} \rightarrow \nabla v$ em $\left(L^{2}(K, \mathbb{R})\right)^{N}$, para qualquer $K \subset \mathbb{R}_{+}^{N}$ compacto. Com efeito, seja $K \subset \mathbb{R}_{+}^{N}$ compacto. Para $\Psi \in C_{c}^{\infty}\left(\mathbb{R}^{N}\right)$ tal que $\Psi \equiv 1$ em $K$ e $\Psi \in[0,1]$, temos $\operatorname{supp} \Psi \subset \tilde{\Omega}_{k}$, para todo $k$

suficientemente grande. Como $v_{k} \Psi, v \Psi \in H^{1}\left(\tilde{\Omega}_{k}, \mathbb{R}\right)$ e $v_{k}$ é uma solução fraca de $\left(P_{5}\right)$, temos

$$
\begin{gathered}
\bar{J}_{\tilde{\Omega}_{k}}^{\prime}\left(v_{k}\right)\left(v_{k} \psi\right)=\int_{\tilde{\Omega}_{k}}\left(\left|\nabla v_{k}\right|^{2} \psi+v_{k} \nabla v_{k} \nabla \psi+v_{k}^{2} \psi\right) \mathrm{d} x-\int_{\tilde{\Omega}_{k}} f\left(v_{k}^{2}\right) v_{k}^{2} \psi \mathrm{d} x=0, \\
\bar{J}_{\tilde{\Omega}_{k}}^{\prime}\left(v_{k}\right)(v \psi)=\int_{\tilde{\Omega}_{k}}\left(\psi \nabla v_{k} \nabla v+v \nabla v_{k} \nabla \psi+v_{k} v \psi\right) \mathrm{d} x-\int_{\tilde{\Omega}_{k}} f\left(v_{k}^{2}\right) v_{k} v \psi \mathrm{d} x=0,
\end{gathered}
$$

onde $\bar{J}_{\tilde{\Omega}_{k}}: H^{1}\left(\tilde{\Omega}_{k}, \mathbb{R}\right) \rightarrow \mathbb{R}$ é o funcional energia associado ao problema $\left(P_{5}\right)$. Combinando (3.28) e (3.29),

$$
\begin{aligned}
0 \leq & \int_{D}\left|\nabla v_{k}-\nabla v\right|^{2} \mathrm{~d} x \leq \int_{\mathbb{R}^{N}} \psi\left(\left|\nabla v_{k}\right|^{2}-2 \nabla v_{k} \nabla v+|\nabla v|^{2}\right) \mathrm{d} x \\
= & \int_{\mathbb{R}_{+}^{N}}\left[\psi\left|\nabla v_{k}\right|^{2}-\psi \nabla v_{k} \nabla v+\psi \nabla v\left(\nabla v-\nabla v_{k}\right)\right] \mathrm{d} x \\
= & \int_{\mathbb{R}_{+}^{N}}\left[f\left(v_{k}^{2}\right) v_{k}^{2} \psi-v_{k} \nabla v_{k} \nabla \psi-v_{k}^{2} \psi\right] \mathrm{d} x+\int_{\mathbb{R}_{+}^{N}}\left[v \nabla v_{k} \nabla \psi+v_{k} v \psi-f\left(v_{k}^{2}\right) v_{k} v \psi\right] \mathrm{d} x \\
& +\int_{\mathbb{R}_{+}^{N}} \psi \nabla v\left(\nabla v-\nabla v_{k}\right) \mathrm{d} x \\
= & \int_{\mathbb{R}_{+}^{N}}\left[f\left(v_{k}^{2}\right) \psi v_{k}\left(v_{k}-v\right)-\left(v_{k}-v\right) \nabla v_{k} \nabla \psi-v_{k} \psi\left(v_{k}-v\right)+\psi \nabla v\left(\nabla v_{k}-\nabla v\right)\right] \mathrm{d} x .
\end{aligned}
$$

Isto e o fato de $\left(v_{k}\right)$ ser limitada em $L^{2}\left(\mathbb{R}_{+}^{N}, \mathbb{R}\right)$ combinado com $\left(f_{2}\right),(3.26)$ e a desigualdade de Hölder, implica

$$
\int_{K}\left|\nabla v_{k}-\nabla v\right|^{2} \mathrm{~d} x \leq o_{k}(1) \text { quando } k \rightarrow \infty
$$

isto é, $\nabla v_{k} \rightarrow \nabla v$ em $\left(L_{l o c}^{2}\left(\mathbb{R}_{+}^{N}, \mathbb{R}\right)\right)^{N}$ quando $k \rightarrow \infty$. Como consequência,

$$
\nabla v_{k}(x) \rightarrow \nabla v(x) \text {, quase sempre em } \mathbb{R}_{+}^{N} \text {, quando } k \rightarrow \infty \text {. }
$$

Para finalizarmos a demonstração, resta-nos ver que

$$
\int_{\mathbb{R}_{+}^{N}}(\nabla v \nabla \phi+v \phi) \mathrm{d} x=\int_{\mathbb{R}_{+}^{N}} f\left(v^{2}\right) v \phi \mathrm{d} x, \quad \forall \phi \in C_{c}^{\infty}\left(\mathbb{R}^{N}\right) .
$$

De fato, como o conjunto das restrições das funções de $C_{c}^{\infty}\left(\mathbb{R}^{N}\right)$ a $\mathbb{R}_{+}^{N}$ é um subespaço denso de $H^{1}\left(\mathbb{R}_{+}^{N}, \mathbb{R}\right)(\operatorname{ver}[13$, Corollaire IX.8]), é suficiente mostramos que (3.31) vale para toda $\phi \in C_{c}^{\infty}\left(\mathbb{R}^{N}\right)$. Dada $\phi \in C_{c}^{\infty}\left(\mathbb{R}^{N}\right)$ qualquer, seja $t>0$ tal que $B_{t}(0) \supset \operatorname{supp} \phi$. Por (3.1), $\chi_{\tilde{\Omega}_{k} \cap B_{t}} \rightarrow \chi_{B_{t}^{+}}$quase sempre em $\mathbb{R}^{N}$, quando $k \rightarrow \infty$, onde $B_{t}^{+}:=B_{t}(0) \cap \mathbb{R}_{+}^{N}$ e $\chi_{B_{t}^{+}}$é a função característica de $B_{t}^{+}$. Isto e (3.30) implicam que $\chi_{\tilde{\Omega}_{k} \cap B_{t}}(x) \nabla v_{k}(x) \rightarrow \chi_{B_{t}^{+}}(x) \nabla v(x)$ 
quase sempre em $\mathbb{R}_{+}^{N}$, quando $k \rightarrow \infty$. Além disso, $\left(\chi_{\tilde{\Omega}_{k} \cap B_{t}} \nabla v_{k}\right)_{k}$ é limitada em $\left(L^{2}\left(\mathbb{R}_{+}^{N}, \mathbb{R}\right)\right)^{N} . \operatorname{Logo} \chi_{\tilde{\Omega}_{k} \cap B_{t}} \nabla v_{k} \rightarrow \chi_{B_{t}^{+}} \nabla v$ em $\left(L^{2}\left(\mathbb{R}_{+}^{N}, \mathbb{R}\right)\right)^{N}$, quando $k \rightarrow \infty$, e daí

$$
\lim _{k \rightarrow \infty} \int_{\tilde{\Omega}_{k}} \nabla v_{k} \nabla \phi \mathrm{d} x=\lim _{k \rightarrow \infty} \int_{\mathbb{R}_{+}^{N}} \chi_{\tilde{\Omega}_{k} \cap B_{t}} \nabla v_{k} \nabla \phi \mathrm{d} x=\int_{\mathbb{R}_{+}^{N}} \chi_{B_{t}^{+}} \nabla v \nabla \phi \mathrm{d} x=\int_{\mathbb{R}_{+}^{N}} \nabla v \nabla \phi \mathrm{d} x .
$$

Usando os limites (3.26) e o fato de $\phi \in C_{c}^{\infty}\left(\mathbb{R}^{N}\right)$,

$$
\int_{\tilde{\Omega}_{k}} v_{k} \phi \mathrm{d} x \rightarrow \int_{\mathbb{R}_{+}^{N}} v \phi \mathrm{d} x, \text { quando } k \rightarrow \infty .
$$

Temos $f\left(v_{k}^{2}\right) v_{k} \rightarrow f\left(v^{2}\right) v$ em $L^{\frac{q}{q-1}}\left(B_{t}^{+}\right)$, pois, por $\left(f_{2}\right), \sup _{k}\left\|v_{k}\right\|_{D_{k}}<\infty$ e imersões de Sobolev, $\sup _{k}\left|f\left(v_{k}^{2}\right) v_{k}\right|_{\frac{q}{q-1}, B_{t}^{+}}<\infty$ e $f\left(v_{k}^{2}(x)\right) v_{k}(x) \rightarrow f\left(v^{2}(x)\right) v(x)$ quase sempre em $\mathbb{R}_{+}^{N}$. Logo, como $\operatorname{supp} \phi \subset B_{t}(0)$ e $\phi \in L^{q}\left(B_{t}^{+}, \mathbb{R}\right)$,

$$
\lim _{k \rightarrow \infty} \int_{\tilde{\Omega}_{k}} f\left(v_{k}^{2}\right) v_{k} \phi=\lim _{k \rightarrow \infty} \int_{B_{t}^{+}} f\left(v_{k}^{2}\right) v_{k} \phi=\int_{B_{t}(0)} f\left(v^{2}\right) v \phi \mathrm{d} x=\int_{\mathbb{R}_{+}^{N}} f\left(v^{2}\right) v \phi \mathrm{d} x .
$$

Por (3.32) - (3.34) e pelo fato de $v_{k}$ satisfazer $\left(P_{5}\right)$,

$$
0=\lim _{k \rightarrow \infty} \int_{\tilde{\Omega}_{k}}\left(\nabla v_{k} \nabla \phi+v_{k} \phi-f\left(v_{k}^{2}\right) v_{k} \phi\right) \mathrm{d} x=\int_{\mathbb{R}_{+}^{N}}\left(\nabla v \nabla \phi+v \phi-f\left(v^{2}\right) v \phi\right) \mathrm{d} x .
$$

Como $\phi \in C_{c}^{\infty}\left(\mathbb{R}^{N}\right)$ é qualquer, $v$ satisfaz $\left(P_{1}\right)$ no sentido fraco.

No que segue, concluiremos a demonstração da Proposição 3.11. Tendo em vista (3.19) e (3.20),

$$
\begin{aligned}
c_{\infty}+o_{k}(1) & \geq \bar{c}_{k}=\bar{c}_{\tilde{\Omega}_{k}} \\
& =\bar{J}_{\tilde{\Omega}_{k}}\left(v_{k}\right)=\bar{J}_{\tilde{\Omega}_{k}}\left(v_{k}\right)-\frac{1}{2} \bar{J}_{\tilde{\Omega}_{k}}^{\prime}\left(v_{k}\right) v_{k} \\
& =\frac{1}{2} \int_{\tilde{\Omega}_{k}}\left[f\left(v_{k}^{2}\right) v_{k}^{2}-F\left(v_{k}^{2}\right)\right] \mathrm{d} x .
\end{aligned}
$$

Pelo Lema de Fatou e (3.26),

$$
\begin{aligned}
c_{\infty} & \geq \limsup _{k \rightarrow \infty} \bar{c}_{\tilde{\Omega}_{k}} \geq \liminf _{k \rightarrow \infty} \bar{c}_{\tilde{\Omega}_{k}} \\
& =\liminf _{k \rightarrow \infty} \frac{1}{2} \int_{\tilde{\Omega}_{k}}\left[f\left(v_{k}^{2}\right) v_{k}^{2}-F\left(v_{k}^{2}\right)\right] \mathrm{d} x \\
& \geq \frac{1}{2} \int_{\mathbb{R}_{+}^{N}}\left[f\left(v^{2}\right) v^{2}-F\left(v^{2}\right)\right] \mathrm{d} x=J_{\infty}(v) \geq c_{\infty} .
\end{aligned}
$$

Portanto, como estes argumentos podem ser aplicados a qualquer subsequência de $\left(\bar{c}_{\lambda}\right)_{\lambda}$,

$$
\lim _{\lambda \rightarrow \infty} \bar{c}_{\lambda}=c_{\infty}
$$


e a prova da Proposição 3.11 está concluída.

O próximo passo é mostrar um resultado de concentração. Antes, façamos algumas considerações.

Seja

$$
m\left(\mathbb{R}_{+}^{N}\right):=\inf _{u \in H^{1}\left(\mathbb{R}_{+}^{N}, \mathbb{R}\right) \backslash\{0\}} \frac{\int_{\mathbb{R}_{+}^{N}}\left(|\nabla u|^{2}+u^{2}\right) \mathrm{d} x}{\left(\int_{\mathbb{R}_{+}^{N}} F\left(u^{2}\right) \mathrm{d} x\right)^{\frac{2}{q}}} .
$$

É fácil mostrar que, definindo $V:=\left\{u \in H^{1}\left(\mathbb{R}_{+}^{N}, \mathbb{R}\right) ; \int_{\mathbb{R}_{+}^{N}} F\left(u^{2}\right) \mathrm{d} x=1\right\}$,

$$
m_{F}\left(\mathbb{R}_{+}^{N}\right)=\inf _{u \in V} \int_{\mathbb{R}_{+}^{N}}\left(|\nabla u|^{2}+u^{2}\right) \mathrm{d} x
$$

Argumentando como no Lema 2.8,

$$
c_{\infty}=\left(\frac{1}{2}-\frac{1}{q}\right)\left(\frac{2}{q}\right)^{\frac{2}{q-2}} m\left(\mathbb{R}_{+}^{N}\right)^{\frac{q}{q-2}} .
$$

Analogamente, temos

$$
\begin{aligned}
m\left(\Omega_{\lambda}, \Gamma_{0 \lambda}\right) & :=\inf _{u \in H^{1}\left(\Omega_{\lambda}, \Gamma_{0 \lambda}\right) \backslash\{0\}} \frac{\int_{\Omega_{\lambda}}\left(|\nabla u|^{2}+u^{2}\right) \mathrm{d} x}{\left(\int_{\Omega_{\lambda}} F\left(u^{2}\right) \mathrm{d} x\right)^{\frac{2}{q}}} \\
& =\inf _{u \in V_{\lambda}} \int_{\Omega_{\lambda}}\left(|\nabla u|^{2}+u^{2}\right) \mathrm{d} x .
\end{aligned}
$$

onde $V_{\lambda}:=\left\{u \in H^{1}\left(\Omega_{\lambda}, \Gamma_{0 \lambda}\right) ; \int_{\Omega_{\lambda}} F\left(u^{2}\right) \mathrm{d} x=1\right\}$, e

$$
c_{\lambda}=\left(\frac{1}{2}-\frac{1}{q}\right)\left(\frac{2}{q}\right)^{\frac{2}{q-2}} m\left(\Omega_{\lambda}, \Gamma_{0 \lambda}\right)^{\frac{q}{q-2}} .
$$

Temos também

$$
m\left(\mathbb{R}^{N}\right):=\inf _{u \in H^{1}\left(\mathbb{R}^{N}, \mathbb{R}\right) \backslash\{0\}} \frac{\int_{\mathbb{R}^{N}}\left(|\nabla u|^{2}+u^{2}\right) \mathrm{d} x}{\left(\int_{\mathbb{R}^{N}} F\left(u^{2}\right) \mathrm{d} x\right)^{\frac{2}{q}}} .
$$

$\mathrm{e}$

$$
c_{\mathbb{R}^{N}}=\left(\frac{1}{2}-\frac{1}{q}\right)\left(\frac{2}{q}\right)^{\frac{2}{q-2}} m\left(\mathbb{R}^{N}\right)^{\frac{q}{q-2}}
$$

Vale ressaltar que, pela Proposição 3.11, (3.37) e (3.40),

$$
\lim _{\lambda \rightarrow \infty} m\left(\Omega_{\lambda}, \Gamma_{0 \lambda}\right)=m\left(\mathbb{R}_{+}^{N}\right) .
$$


e, usando (3.9), (3.37) e (3.42),

$$
m\left(\mathbb{R}_{+}^{N}\right)<m\left(\mathbb{R}^{N}\right)
$$

Lema 3.14. Sejam, para todo $\lambda>0, w_{\lambda} \in V_{\lambda}$. Se

$$
\lim _{\lambda \rightarrow \infty}\left\|w_{\lambda}\right\|_{\Omega_{\lambda}}^{2}=m_{F}\left(\mathbb{R}_{+}^{N}\right),
$$

então dado $\epsilon>0$, existem $y_{\lambda} \in \Gamma_{1 \lambda}$ e $R>0$ tais que

$$
\lim _{\lambda \rightarrow \infty} \int_{\Omega_{\lambda} \cap B_{R}\left(y_{\lambda}\right)} F\left(w_{\lambda}^{2}\right) \mathrm{d} x \geq 1-\epsilon
$$

\section{Demonstração.}

Usaremos o Lema de Concentração-Compacidade de Lions [31] para concluirmos que vale a concentração para a sequência de medidas $\mu_{\lambda}:=\chi_{\Omega_{\lambda}} F\left(w_{\lambda}^{2}\right)$. Para isto, precisamos descartar o anulamento e a dicotomia. É fácil ver que o anulamento não ocorre, uma vez que $w_{\lambda} \in V_{\lambda}$, para todo $\lambda>0$. Vejamos que a dicotomia também não ocorre. Suponhamos, por contradição, que temos dicotomia. Então existe $\alpha \in(0,1)$ tal que

$$
\lim _{t \rightarrow \infty} Q(t)=\alpha,
$$

onde

$$
Q(t):=\lim _{\lambda \rightarrow \infty} Q_{\lambda}(t), \quad \text { e } \quad Q_{\lambda}(t):=\sup _{y \in \mathbb{R}^{N}} \int_{y+B_{t}} \chi_{\Omega_{\lambda}} F\left(w_{\lambda}^{2}\right) \mathrm{d} x .
$$

Note que $Q$ é uma função decrescente e $Q_{\lambda}$ é uma função contínua.

Por (3.45), dado $\epsilon>0$, existe $R_{0}>0$ tal que

$$
\alpha \geq Q\left(R_{0}\right) \geq \alpha-\frac{\epsilon}{4}
$$

Por (3.46) e pelas definições de $Q$ e $Q_{\lambda}$, existem, para cada $\lambda>0, y_{\lambda} \in \mathbb{R}^{N}$ e $\lambda_{0}>0$ tais que

$$
Q_{\lambda}\left(R_{0}\right) \geq \int_{y_{\lambda}+B_{R_{0}}} \chi_{\Omega_{\lambda}} F\left(w_{\lambda}^{2}\right) \mathrm{d} x \geq \alpha-\frac{\epsilon}{2}, \quad \forall \lambda \geq \lambda_{0} .
$$

Atente também para o fato de que existem $R_{k} \rightarrow \infty$ quando $k \rightarrow \infty$ e uma subsequência $Q_{\lambda_{k}}$ tais que

$$
Q_{\lambda_{k}}\left(R_{k}\right) \leq \alpha+\frac{\epsilon}{2}
$$

De fato, fixe $R_{\lambda} \rightarrow \infty$ qualquer. Então, por (3.45), $\lim _{\lambda \rightarrow \infty} Q\left(2 R_{\lambda}\right)=\alpha$. Existe uma subsequência, que denotaremos por $\left(R_{k}\right)$, tal que

$$
\left|Q\left(R_{k}\right)-\alpha\right| \leq \frac{1}{2 k}, \quad \forall k \in \mathbb{N} .
$$

Como, para cada $k$ fixo, $\lim _{\lambda \rightarrow \infty} Q_{\lambda}\left(2 R_{k}\right)=Q\left(2 R_{k}\right)$, existe subsequência $Q_{\lambda_{k}}$ tal que

$$
\left|Q_{\lambda_{k}}-Q\left(2 R_{k}\right)\right| \leq \frac{1}{2 k}, \quad \forall k \in \mathbb{N} .
$$


Logo, por (3.48)-(3.49),

$$
Q_{\lambda_{k}}\left(2 R_{k}\right) \leq\left|Q_{\lambda_{k}}\left(2 R_{k}\right)-Q\left(2 R_{k}\right)\right|+\left|Q\left(2 R_{k}\right)-\alpha\right|+\alpha \leq \frac{1}{k}+\alpha, \quad \forall k \in \mathbb{N},
$$

ou seja, para $k$ suficientemente grande,

$$
Q_{\lambda_{k}}\left(2 R_{k}\right) \leq \alpha+\frac{\epsilon}{2}
$$

Denotando a subsequência $\left(Q_{\lambda_{k}}\right)$ por $\left(Q_{k}\right)$, temos, para todo $k$ suficientemente grande,

$$
Q_{k}\left(2 R_{k}\right) \leq \alpha+\frac{\epsilon}{2}
$$

Vale ressaltar que, por (3.47), para todo $k$ suficientemente grande,

$$
Q_{k}\left(R_{0}\right) \geq \int_{y_{k}+B_{R_{0}}} \chi_{k} F\left(w_{k}^{2}\right) \mathrm{d} x \geq \alpha-\frac{\epsilon}{2}
$$

onde $\chi_{k}:=\chi_{\Omega_{\lambda_{k}}}$.

Considere $\eta \in C^{\infty}\left(\mathbb{R}_{+}\right)$a aplicação definida anteriormente e seja

$$
\xi:=1-\eta
$$

Além disso, sejam

$$
w_{k}^{1}(x):=\chi_{k}(x) \eta\left(\frac{\left|x-y_{k}\right|}{R_{0}}\right) w_{k}(x), \quad w_{k}^{2}(x):=\chi_{k}(x) \xi\left(\frac{\left|x-y_{k}\right|}{R_{k}}\right) w_{k}(x), \quad \forall x \in \mathbb{R}^{N} .
$$

Vale atentar para o fato de que $w_{k}^{j} \in H^{1}\left(\Omega_{\lambda_{k}}, \Gamma_{0 \lambda_{k}}\right), j=1,2$. Temos, por (3.51),

$$
\int_{\mathbb{R}^{N}} F\left(w_{k}^{12}\right) \mathrm{d} x \geq \alpha-\frac{\epsilon}{2}
$$

e, por (3.50),

$$
\int_{y_{k}+B_{2 R_{k}}} \chi_{k} F\left(w_{k}^{2}\right) \mathrm{d} x \leq \alpha+\frac{\epsilon}{2},
$$

donde

$$
\int_{y_{k}+B_{2 R_{k}}^{c}} \chi_{k} F\left(w_{k}^{2}\right) \mathrm{d} x \geq 1-\alpha-\frac{\epsilon}{2}
$$

e consequentemente

$$
\int_{\mathbb{R}^{N}} F\left(w_{k}^{2}\right) \mathrm{d} x \geq 1-\alpha-\frac{\epsilon}{2}
$$

Logo, por (3.52)-(3.53), para $k$ suficientemente grande,

$$
\begin{aligned}
0 \leq 1-\int_{\mathbb{R}^{N}}\left(F\left(w_{k}^{12}\right)+F\left(w_{k}^{2}\right)\right) \mathrm{d} x & \leq 1-\alpha+\frac{\epsilon}{2}-1+\alpha+\frac{\epsilon}{2} \\
& \leq \epsilon .
\end{aligned}
$$


Temos também

$$
\begin{gathered}
\int_{\mathbb{R}^{N}} F\left(w_{k}^{12}\right) \mathrm{d} x-\alpha \leq \frac{\epsilon}{2}, \\
\int_{\mathbb{R}^{N}} F\left(w_{k}^{2}\right) \mathrm{d} x-(1-\alpha) \leq \frac{3 \epsilon}{2} .
\end{gathered}
$$

Com efeito, (3.55) segue de (3.52), donde, tendo em vista (3.54),

$$
\begin{aligned}
\int_{\mathbb{R}^{N}} F\left(w_{k}^{2}\right) \mathrm{d} x-(1-\alpha) & \leq \int_{\mathbb{R}^{N}} F\left(w_{k}^{12}\right) \mathrm{d} x+\int_{\mathbb{R}^{N}} F\left(w_{k}^{22}\right) \mathrm{d} x-1+\int_{\mathbb{R}^{N}} F\left(w_{k}^{12}\right) \mathrm{d} x+\alpha \\
& \leq \epsilon+\frac{\epsilon}{2}=\frac{3 \epsilon}{2} .
\end{aligned}
$$

Temos também, para alguma constante $C>0$ adequada, por um cálculo simples,

$$
\int_{\Omega_{\lambda_{k}}}\left|\nabla w_{k}^{1}\right|^{2} \mathrm{~d} x \leq \int_{\Omega_{\lambda_{k}}}\left|\nabla w_{k}\right|^{2} \eta^{2}\left(\frac{\left|x-y_{k}\right|}{R_{0}}\right) \mathrm{d} x+\frac{C}{R_{0}^{2}}+\frac{C}{R_{0}}
$$

$\mathrm{e}$

$$
\int_{\Omega_{\lambda_{k}}}\left|\nabla w_{k}^{2}\right|^{2} \mathrm{~d} x \leq \int_{\Omega_{\lambda_{k}}}\left|\nabla w_{k}\right|^{2} \xi^{2}\left(\frac{\left|x-y_{k}\right|}{R_{k}}\right) \mathrm{d} x+\frac{C}{R_{k}^{2}}+\frac{C}{R_{k}} .
$$

Portanto, para $R_{k}>2 R_{0}, R_{0}$ suficientemente grande,

$$
\begin{aligned}
\left\|w_{k}^{1}\right\|_{\Omega_{\lambda_{k}}}^{2}+\left\|w_{k}^{2}\right\|_{\Omega_{\lambda_{k}}}^{2} \leq & \int_{\Omega_{\lambda_{k}}}\left[\eta^{2}\left(\frac{\left|x-y_{k}\right|}{R_{0}}\right)+\xi^{2}\left(\frac{\left|x-y_{k}\right|}{R_{k}}\right)\right]\left(\left|\nabla w_{k}\right|^{2}+w_{k}^{2}\right) \mathrm{d} x \\
& +C\left(\frac{1}{R_{0}}+\frac{1}{R_{0}^{2}}+\frac{1}{R_{k}}+\frac{1}{R_{k}^{2}}\right) \\
\leq & \left\|w_{k}\right\|_{\Omega_{\lambda_{k}}}^{2}+\epsilon .
\end{aligned}
$$

Agora, por (3.55)-(3.57), tendo em vista (3.38) e (3.43),

$$
\begin{aligned}
m\left(\mathbb{R}_{+}^{N}\right) & =\lim _{k \rightarrow \infty} \int_{\Omega_{\lambda_{k}}}\left(\left|\nabla w_{k}\right|^{2}+w_{k}^{2}\right) \mathrm{d} x \\
& \geq \lim _{k \rightarrow \infty}\left(\int_{\Omega_{\lambda_{k}}}\left(\left|\nabla w_{k}^{1}\right|^{2}+w_{k}^{1^{2}}\right) \mathrm{d} x+\int_{\Omega_{\lambda_{k}}}\left(\left|\nabla w_{k}^{2}\right|^{2}+w_{k}^{22}\right) \mathrm{d} x-\epsilon\right) \\
& \geq \lim _{k \rightarrow \infty}\left(\left(\int_{\mathbb{R}^{N}} F\left(w_{k}^{1^{2}}\right) \mathrm{d} x\right)^{\frac{2}{q}}+\left(\int_{\mathbb{R}^{N}} F\left(w_{k}^{22}\right) \mathrm{d} x\right)^{\frac{2}{q}}\right) m\left(\Omega_{\lambda_{k}}, \Gamma_{0 \lambda_{k}}\right)-\epsilon \\
& \geq m\left(\mathbb{R}_{+}^{N}\right)\left[\left(\alpha-\frac{\epsilon}{2}\right)^{\frac{2}{q}}+\left(1-\alpha-\frac{3 \epsilon}{2}\right)^{\frac{2}{q}}-\frac{\epsilon}{m\left(\mathbb{R}_{+}^{N}\right)}\right]
\end{aligned}
$$

donde, fazendo $\epsilon \rightarrow 0$, uma vez que $\alpha \in(0,1)$,

$$
1 \geq(1-\alpha)^{\frac{2}{q}}+\alpha^{\frac{2}{q}}>1
$$

o que é um absurdo. Portanto a dicotomia também não ocorre. Sendo assim, pelo Lema de Concentração-Compacidade de Lions, dado $\epsilon>0$, existem $R>0$ e sequência $\left(y_{\lambda}\right) \subset \mathbb{R}^{N}$ tais que

$$
\lim _{\lambda \rightarrow \infty} \int_{\Omega_{\lambda} \cap B_{R}\left(y_{\lambda}\right)} F\left(w_{\lambda}^{2}\right) \mathrm{d} x \geq 1-\epsilon
$$


76 Capítulo 3 - Equações de Schrödinger com condição de fronteira mista: caso homogêneo

Sobre a sequência $\left(y_{\lambda}\right)$, primeiramente notemos que, aumentando $R$ se necessário, sem perder a generalidade, podemos supor que $y_{\lambda} \in \Omega_{\lambda}$, para todo $\lambda>0$, uma vez que (3.58) implica em $\Omega_{\lambda} \cap B_{R}\left(y_{\lambda}\right) \neq \emptyset$. Vejamos que $y_{\lambda} \in \partial \Omega_{\lambda}$. Seja $\epsilon>0$ qualquer. Vale (3.58). Suponha, por contradição, que

$$
\alpha_{\lambda}:=\operatorname{dist}\left(y_{\lambda}, \partial \Omega_{\lambda}\right) \rightarrow \infty \text {, quando } \lambda \rightarrow \infty
$$

Sejam, então, as sequências $\left(R_{\lambda}\right)_{\lambda} \subset \mathbb{R}$ dada por $R_{\lambda}:=\frac{\alpha_{\lambda}}{2}$ e

$$
\tilde{w}_{\lambda}(x):=\eta\left(\frac{\left|x-y_{\lambda}\right|}{R_{\lambda}}\right) w_{\lambda}(x), \quad \forall x \in \Omega_{\lambda} .
$$

Note que $\tilde{w}_{\lambda} \in H_{0}^{1}\left(\Omega_{\lambda}, \mathbb{R}\right)$, para todo $\lambda>0$. Mais ainda, estendendo $\tilde{w}_{\lambda}$ por zero fora de $\Omega_{\lambda}$ para cada $\lambda>0,\left(\tilde{w}_{\lambda}\right)_{\lambda} \subset H^{1}\left(\mathbb{R}^{N}, \mathbb{R}\right)$. Além disso, para $\lambda$ suficientemente grande de modo que $R_{\lambda}>R$,

$$
\int_{\mathbb{R}^{N}} F\left(\tilde{w}_{\lambda}^{2}\right) \mathrm{d} x \geq \int_{\Omega_{\lambda} \cap B_{R}\left(y_{\lambda}\right)} F\left(w_{\lambda}^{2}\right) \mathrm{d} x \geq 1-\epsilon .
$$

Argumentando como em (3.57),

$$
\left\|w_{\lambda}\right\|_{\Omega_{\lambda}}^{2}+\epsilon \geq\left\|\tilde{w}_{\lambda}\right\|^{2}
$$

Daí, usando a nossa hipótese, (3.41) e (3.58),

$$
m\left(\mathbb{R}_{+}^{N}\right)+o_{\lambda}(1)+\epsilon \geq m\left(\mathbb{R}^{N}\right)\left(\int_{\mathbb{R}^{N}} F\left(\tilde{w}_{\lambda}^{2}\right) \mathrm{d} x\right)^{\frac{2}{q}} \geq m\left(\mathbb{R}^{N}\right)(1-\epsilon)^{\frac{2}{q}} .
$$

Portanto, tomando o limite $\epsilon \rightarrow 0$,

$$
m\left(\mathbb{R}_{+}^{N}\right)+o_{\lambda}(1) \geq m\left(\mathbb{R}^{N}\right)
$$

o que, tendo em vista (3.44), é uma contradição. Logo $\left(\operatorname{dist}\left(y_{\lambda}, \partial \Omega_{\lambda}\right)\right)_{\lambda}$ é uma sequência limitada. Aumentando $R$ se necessário, podemos supor, sem perder a generalidade, que $y_{\lambda} \in \partial \Omega_{\lambda}$, para todo $\lambda>0$. Por fim, vejamos que, para todo $\lambda>0$,

$$
y_{\lambda} \in \Gamma_{1 \lambda} .
$$

Argumentando novamente por contradição, suponha que

$$
\delta_{\lambda}:=\operatorname{dist}\left(y_{\lambda}, \Gamma_{1 \lambda}\right) \rightarrow \infty, \text { quando } \lambda \rightarrow \infty \text {. }
$$

Sejam as sequências $\left(R_{\lambda}\right)_{\lambda} \subset \mathbb{R}$ dada por $R_{\lambda}:=\frac{\delta_{\lambda}}{3}$ e

$$
\hat{w}_{\lambda}(x):=\eta\left(\frac{\left|x-y_{\lambda}\right|}{R_{\lambda}}\right) w_{\lambda}(x), \quad \forall x \in \Omega_{\lambda} .
$$

Note que, pela forma como $R_{\lambda}$ e $\eta$ foram tomadas, $\hat{w}_{\lambda} \in H_{0}^{1}\left(\Omega_{\lambda}, \mathbb{R}\right)$, para todo $\lambda>0$. Mais ainda, estendendo $\hat{w}_{\lambda}$ por zero fora de $\Omega_{\lambda}$ para cada $\lambda>0,\left(\hat{w}_{\lambda}\right)_{\lambda} \subset H^{1}\left(\mathbb{R}^{N}, \mathbb{R}\right)$. Seguindo 
os mesmos passos do caso anterior, chegamos a um absurdo idêntico. Isto prova que, para todo $\lambda>0$,

$$
y_{\lambda} \in \Gamma_{1 \lambda}
$$

e o lema está demonstrado.

\subsection{A aplicação baricentro}

Definamos, para cada $\lambda>0$, a aplicação baricentro $\beta_{\lambda}: M_{\lambda} \rightarrow \mathbb{R}^{N}$ por

$$
\beta_{\lambda}(u)=\frac{\int_{\Omega_{\lambda}} x F\left(|u|^{2}\right) \mathrm{d} x}{\int_{\Omega_{\lambda}} F\left(|u|^{2}\right) \mathrm{d} x}
$$

Proposição 3.15. Existem $\epsilon^{*}>0, \lambda_{1}>0$ tais que $\beta_{\lambda}(u) \in \Gamma_{1 \lambda}^{+}$, desde que $\lambda>\lambda_{1}, u \in M_{\lambda}$ e $I_{\lambda}(u) \leq b_{\lambda}^{*}$, onde $b_{\lambda}^{*}:=b_{\lambda}+\epsilon^{*}$.

\section{Demonstração.}

Sejam $\left(\epsilon_{n}\right)_{n},\left(\lambda_{n}\right)_{n}$ e $u_{n} \in M_{\lambda_{n}}$ tais que $\epsilon_{n} \rightarrow 0, \lambda_{n} \rightarrow \infty$ quando $n \rightarrow \infty$, e

$$
I_{\lambda_{n}}\left(u_{n}\right) \leq b_{\lambda_{n}}+\epsilon_{n}
$$

Mostremos que, para $n$ suficientemente grande,

$$
\operatorname{dist}\left(\beta_{\lambda_{n}}\left(u_{n}\right), \Gamma_{1 \lambda_{n}}\right) \leq \lambda_{n} r
$$

Como $u_{n} \in M_{\lambda_{n}}$,

$$
b_{\lambda_{n}} \leq I_{\lambda_{n}}\left(u_{n}\right) \leq b_{\lambda_{n}}+\epsilon_{n}
$$

e daí, pela Proposição 3.11,

$$
I_{\lambda_{n}}\left(u_{n}\right) \rightarrow c_{\infty} \text { quando } n \rightarrow \infty
$$

Note que, pela desigualdade diamagnética, pelo fato de $u_{n} \in M_{\lambda_{n}}$ e pelas características de $f$, existe $t_{n}>0$ tal que

$$
o_{n}(1)+c_{\infty}=I_{\lambda_{n}}\left(u_{n}\right) \geq \max _{t \geq 0} I_{\lambda_{n}}\left(t u_{n}\right) \geq \max _{t \geq 0} J_{\lambda_{n}}\left(t\left|u_{n}\right|\right)=J_{\lambda_{n}}\left(t_{n}\left|u_{n}\right|\right) \geq c_{\lambda_{n}},
$$

onde $J_{\lambda_{n}}, c_{\lambda_{n}}$ e $N_{\lambda_{n}}$ são, respectivamente, o funcional energia, o nível de energia mínima e a variedade Nehari associados ao problema $\left(P_{3}\right)$.

Ainda pela Proposição 3.11,

$$
c_{\infty}=\lim _{n \rightarrow \infty} b_{\lambda_{n}} \geq \lim _{n \rightarrow \infty} c_{\lambda_{n}} \geq \lim _{n \rightarrow \infty} \bar{c}_{\lambda_{n}}=c_{\infty}
$$


Assim

$$
\lim _{n \rightarrow \infty} c_{\lambda_{n}}=\lim _{n \rightarrow \infty} J_{\lambda_{n}}\left(t_{n}\left|u_{n}\right|\right)=c_{\infty}
$$

A ideia é aplicarmos o Lema 3.14 à sequência

$$
w_{n}:=\frac{t_{n}\left|u_{n}\right|}{\left(\int_{\Omega_{\lambda_{n}}} F\left(t_{n}^{2}\left|u_{n}\right|^{2}\right) \mathrm{d} x\right)^{\frac{1}{q}}}
$$

Mostremos, então, que esta sequência satisfaz as hipóteses do referido lema.

Usando a homogeneidade de $F$, é fácil mostrar que $w_{n} \in V_{\lambda_{n}}$, para todo $n \in \mathbb{N}$. Além disso, repetindo os argumentos usados para concluirmos (3.37), temos, quando $n \rightarrow \infty$

$$
\left\|w_{n}\right\|_{\Omega_{\lambda_{n}}}^{2} \rightarrow m\left(\mathbb{R}_{+}^{N}\right)
$$

Dessa forma, dado $\epsilon>0$, existem $R>0$ e sequência $y_{n} \in \Gamma_{1 \lambda_{n}}$ tais

$$
\lim _{n \rightarrow \infty} \frac{\int_{\Omega_{\lambda_{n}} \cap B_{R}\left(y_{n}\right)} F\left(t_{n}^{2}\left|u_{n}\right|^{2}\right) \mathrm{d} x}{\int_{\Omega_{\lambda_{n}}} F\left(t_{n}^{2}\left|u_{n}\right|^{2}\right) \mathrm{d} x} \geq \lim _{\lambda \rightarrow \infty} \int_{\Omega_{\lambda} \cap B_{R}\left(y_{n}\right)} F\left(w_{n}^{2}\right) \mathrm{d} x \geq 1-\epsilon .
$$

Por fim, mostremos que

$$
\lim _{n \rightarrow \infty} \operatorname{dist}\left(\frac{\beta_{\lambda_{n}}\left(u_{n}\right)}{\lambda_{n}}, \Gamma_{1}\right)=0 .
$$

De fato, primeiramente note que, pela homogeneidade de $F$ e pela definição de $\beta_{\lambda_{n}}$,

$$
\beta_{\lambda_{n}}\left(u_{n}\right)=\beta_{\lambda_{n}}\left(t_{n}\left|u_{n}\right|\right) .
$$

Observemos que $\frac{y_{n}}{\lambda_{n}} \in \Gamma_{1}$, para todo $n \in \mathbb{N}$. Assim, existe $x_{0} \in \bar{\Gamma}_{1}$ tal que $\frac{y_{n}}{\lambda_{n}} \rightarrow x_{0}$ quando $n \rightarrow \infty$, a menos de subsequência. Temos, para cada $j \in\{1, \ldots, N\}$,

$$
\begin{aligned}
\left|\frac{\beta_{\lambda_{n}}^{j}\left(u_{n}\right)}{\lambda_{n}}-x_{0}^{j}\right| & =\left|\frac{\beta_{\lambda_{n}}^{j}\left(t_{n}\left|u_{n}\right|\right)}{\lambda_{n}}-x_{0}^{j}\right| \\
& =\left|\frac{\int_{\Omega_{\lambda_{n}}} \frac{x^{j}}{\lambda_{n}} F\left(t_{n}^{2}\left|u_{n}\right|^{2}\right) \mathrm{d} x}{\int_{\Omega_{\lambda_{n}}} F\left(t_{n}^{2}\left|u_{k}\right|^{2}\right) \mathrm{d} x}-\frac{\int_{\Omega_{\lambda_{n}}} x_{0}^{j} F\left(t_{n}^{2}\left|u_{n}\right|^{2}\right) \mathrm{d} x}{\int_{\Omega_{\lambda_{n}}} F\left(t_{n}^{2}\left|u_{n}\right|^{2}\right) \mathrm{d} x}\right| \\
& \leq \frac{\int_{\Omega_{\lambda_{n}}}\left|\frac{x^{j}}{\lambda_{n}}-x_{0}^{j}\right| F\left(t_{n}^{2}\left|u_{n}\right|^{2}\right) \mathrm{d} x}{\int_{\Omega_{\lambda_{n}}} F\left(t_{n}^{2}\left|u_{n}\right|^{2}\right) \mathrm{d} x} .
\end{aligned}
$$

Observemos que, como $t_{n}\left|u_{n}\right| \in N_{\lambda_{n}}$, argumentando como na Proposição 3.6 e por $\left(f_{1}\right)$, sem perda de generalidade,

$$
\int_{\Omega_{\lambda_{n}}} F\left(t_{n}^{2}\left|u_{n}\right|^{2}\right) \geq \delta_{0}>0, \quad \forall n \in \mathbb{N} .
$$


Assim,

$$
\begin{aligned}
\delta_{0}\left|\frac{\beta_{\lambda_{n}}^{j}\left(u_{n}\right)}{\lambda_{n}}-x_{0}^{j}\right| \leq & \left|\frac{\beta_{\lambda_{n}}^{j}\left(u_{n}\right)}{\lambda_{n}}-x_{0}^{j}\right| \int_{\Omega_{\lambda_{n}}} F\left(t_{n}^{2}\left|u_{n}\right|^{2}\right) \mathrm{d} x \leq \int_{\Omega_{\lambda_{n}}}\left|\frac{x^{j}}{\lambda_{n}}-x_{0}^{j}\right| F\left(t_{n}^{2}\left|u_{n}\right|^{2}\right) \mathrm{d} x \\
\leq & \left(\int_{\Omega_{\lambda_{n}} \cap B_{R}\left(y_{n}\right)}+\int_{\Omega_{\lambda_{n}} \backslash B_{R}\left(y_{n}\right)}\right)\left|\frac{x^{j}}{\lambda_{n}}-x_{0}^{j}\right| F\left(t_{n}^{2}\left|u_{n}\right|^{2}\right) \mathrm{d} x \\
\leq & \int_{\Omega_{\lambda_{n}} \cap B_{R}\left(y_{n}\right)}\left|\frac{x^{j}}{\lambda_{n}}-\frac{y_{n}^{j}}{\lambda_{n}}\right| F\left(t_{n}^{2}\left|u_{n}\right|^{2}\right) \mathrm{d} x+ \\
& +\int_{\Omega_{\lambda_{n}} \cap B_{R}\left(y_{n}\right)}\left|\frac{y_{n}^{j}}{\lambda_{n}}-x_{0}^{j}\right| F\left(t_{n}^{2}\left|u_{n}\right|^{2}\right) \mathrm{d} x+ \\
& +\int_{\Omega_{\lambda_{n}} \backslash B_{R}\left(y_{n}\right)}\left|\frac{x^{j}}{\lambda_{n}}-x_{0}^{j}\right| F\left(t_{n}^{2}\left|u_{n}\right|^{2}\right) \mathrm{d} x \\
\leq & \frac{R}{\lambda_{n}} \int_{\Omega_{\lambda_{n}}} F\left(t_{n}^{2}\left|u_{n}\right|^{2}\right) \mathrm{d} x+\left|\frac{y_{n}^{j}}{\lambda_{n}}-x_{0}\right| \int_{\Omega_{\lambda_{n}}} F\left(t_{n}^{2}\left|u_{n}\right|^{2}\right) \mathrm{d} x+ \\
& +\operatorname{diam}(\Omega) \int_{\Omega_{\lambda_{n}} \backslash B_{R}\left(y_{n}\right)} F\left(t_{n}^{2}\left|u_{n}\right|^{2}\right) \mathrm{d} x \\
= & \left(\frac{R}{\lambda_{n}}+\left|\frac{y_{n}^{j}}{\lambda_{n}}-x_{0}\right|\right) \int_{\Omega_{n}} F\left(t_{n}^{2}\left|u_{n}\right|^{2}\right) \mathrm{d} x+ \\
& +\operatorname{diam}(\Omega) \int_{\Omega_{\lambda_{n}} \backslash B_{R}\left(y_{n}\right)} F\left(t_{n}^{2}\left|u_{n}\right|^{2}\right) \mathrm{d} x
\end{aligned}
$$

e passando ao limite $n \rightarrow \infty$, por (3.63) e pelo fato de $\left(\left\|t_{n} u_{n}\right\|_{\Omega_{\lambda_{n}}}\right)_{n}$ ser limitada,

$$
0 \leq \lim _{n \rightarrow \infty}\left|\frac{\beta_{\lambda_{n}}^{j}\left(u_{n}\right)}{\lambda_{n}}-x_{0}^{j}\right| \leq \operatorname{diam}(\Omega) \frac{\epsilon C}{\delta_{0}},
$$

para alguma constante $C>0$, donde, da arbitrariedade da escolha de $\epsilon>0$, a menos de subsequência, vale (3.64). Assim, para $n$ suficientemente grande,

$$
\operatorname{dist}\left(\beta_{\lambda_{n}}\left(u_{n}\right), \Gamma_{1 \lambda_{n}}\right) \leq \lambda_{n} r .
$$

Como os argumentos empregados podem ser aplicados a quaisquer subsequências das sequências tomadas inicialmente, segue o resultado.

Para $\epsilon^{*}$ dado no Lema 3.15. Definamos

$$
b_{\lambda}^{*}=b_{\lambda}+\epsilon^{*}
$$

Lema 3.16. Existe $\lambda^{*}>0$ tal que, para $\lambda>\lambda^{*}$,

$$
\Phi_{\lambda}\left(\Gamma_{\lambda 1}^{-}\right) \subset M_{\lambda}^{b_{\lambda}^{*}}, \quad \beta_{\lambda}\left(M_{\lambda}^{b_{\lambda}^{*}}\right) \subset \Gamma_{\lambda 1}^{+},
$$

onde $M_{\lambda}^{b_{\lambda}^{*}}:=I_{\lambda}^{b_{\lambda}^{*}} \cap M_{\lambda}$. 


\section{Demonstração.}

Temos, pelo Lema 3.9 e Proposição 3.11,

$$
\lim _{\lambda \rightarrow \infty} \max _{y \in \Gamma_{1 \lambda}^{-}}\left|I_{\lambda}\left(\Phi_{\lambda}(y)\right)-b_{\lambda}\right|=0 .
$$

Pela definição de limite, (3.66) implica que, para $\epsilon^{*}>0$, existe $\lambda_{2}>0$ tal que, para $\lambda>\lambda_{2}$,

$$
I_{\lambda}\left(\Phi_{\lambda}(y)\right) \leq b_{\lambda}+\epsilon^{*}
$$

para qualquer $y \in \Gamma_{1 \lambda}^{-}$. Ponhamos $\lambda^{*}:=\max \left\{\lambda_{1}, \lambda_{2}\right\}$. Então, das observações acima, temos, para $\lambda>\lambda^{*}$

$$
\Phi_{\lambda}\left(\Gamma_{1 \lambda}^{-}\right) \subset M_{\lambda}^{b_{\lambda}^{*}} \quad \text { e } \quad \beta_{\lambda}\left(M_{\lambda}^{b_{\lambda}^{*}}\right) \subset \Gamma_{1 \lambda}^{+}
$$

Vale ressaltar que, pelo lema acima, $\beta \circ \Phi_{\lambda}$ é homotopicamente equivalente à identidade em $\Gamma_{1}$. Na próxima seção, veremos os resultados de categoria de Ljusternik-Schnirelmann para o funcional $I_{\lambda}$ e a demonstração do Teorma 3.1.

\subsection{Prova do Teorema 3.1}

Através das aplicações $\beta_{\lambda}$ e $\Phi_{\lambda}$ definidas anteriormente, é possível compararmos as topologias de $M_{\lambda}^{b_{\lambda}^{*}}$ e $\Gamma_{1 \lambda}$.

Lema 3.17. Seja $\lambda^{*}>0$ dado no Lema 3.16. Então, para qualquer $\lambda>\lambda^{*}$,

$$
\operatorname{cat}\left(M_{\lambda}^{b_{\lambda}^{*}}\right) \geq \operatorname{cat}\left(\Gamma_{1 \lambda}\right) .
$$

\section{Demonstração.}

Suponha que $\operatorname{cat}\left(M_{\lambda}^{b_{\lambda}^{*}}\right)=m$. Então existem $\Upsilon_{j} \subset M_{\lambda}^{b_{\lambda}^{*}}, j=1, \ldots, m$, fechados e contráteis em $M_{\lambda}^{b_{\lambda}^{*}}$, tais que

$$
M_{\lambda}^{b_{\lambda}^{*}}=\bigcup_{j=1}^{m} \Upsilon_{j}
$$

e $h_{j} \in C\left([0,1] \times \Upsilon_{j}, M_{\lambda}^{b_{\lambda}^{*}}\right)$ tais que

$$
h_{j}(0, u)=u, h_{j}(1, u)=u_{j} \in M_{\lambda}^{b_{\lambda}^{*}}, \text { para cada } u \in \Upsilon_{j}, j=1, \ldots, m,
$$

e $u_{j} \in M_{\lambda}^{b_{\lambda}^{*}}$ fixos. Considere os conjuntos $B_{j}:=\Phi_{\lambda}^{-1}\left(\Upsilon_{j}\right), j=1, \ldots, m$, os quais são fechados em $\Gamma_{1 \lambda}^{-}$e, pela Proposição 3.16,

$$
\Gamma_{1 \lambda}^{-}=\bigcup_{j=1}^{m} B_{j} .
$$


Pela Proposição 3.16, as aplicações $g_{j}:[0,1] \times B_{j} \rightarrow \Gamma_{1 \lambda}^{+}$dadas por

$$
g_{j}(t, y):=\beta_{\lambda}\left(h_{j}\left(t, \Phi_{\lambda}(y)\right)\right), \quad \forall j \in\{1, \ldots, m\},
$$

estão bem definidas. Mais ainda, $g_{j} \in C\left([0,1] \times B_{j}, \Gamma_{1 \lambda}^{+}\right)$e

$$
g_{j}(0, y)=y, g_{j}(1, y)=y_{j} \in \Gamma_{1 \lambda}^{+}, \text {para cada } y \in B_{j}, j=1, \ldots, m,
$$

e $y_{j} \in \Gamma_{1 \lambda}^{+}$fixos. Portanto, como $\Gamma_{1 \lambda}^{+}$e $\Gamma_{1 \lambda}^{-}$são homotopicamente equivalentes a $\Gamma_{1 \lambda}$,

$$
\operatorname{cat} \Gamma_{1 \lambda}=\operatorname{cat}_{\Gamma_{1 \lambda}^{+}} \Gamma_{1 \lambda}^{-} \leq m,
$$

como queríamos.

\section{Demonstração do Teorema 3.1.}

Tomemos $\epsilon^{*}>0$ dado na Proposição $3.15, \lambda^{*}>0$ dado na Proposição 3.16 e $\lambda>\lambda^{*}$. Se $b_{\lambda}^{*}=b_{\lambda}+\epsilon$ é um valor crítico de $I_{\lambda}$ para todo $\epsilon \in\left(0, \epsilon^{*}\right]$, então $I_{\lambda}$ possui infinitos pontos críticos e a prova está completa. Por outro lado, admitamos que $b_{\lambda}^{*}$ é um valor regular de $I_{\lambda}$. Como $M_{\lambda}^{b_{\lambda}^{*}}$ é um conjunto fechado de $M_{\lambda}$, tendo em vista as Proposição 3.7, $I_{\lambda}$ restrito a $M_{\lambda}^{b_{\lambda}^{*}}$ satisfaz $(P S)_{d}$, para todo $d \in \mathbb{R}$. Logo podemos aplicar a teoria de Ljusternik-Schnirelmann e a Proposição 3.17 para obtemos $\operatorname{cat}\left(\Gamma_{1 \lambda}\right)$ pontos críticos de $\left.I_{\lambda}\right|_{M_{\lambda}^{b_{\lambda}^{*}}}$. Pelo fato de $b_{\lambda}^{*}$ ser valor regular, obtemos $\operatorname{cat}\left(\Gamma_{1 \lambda}\right)$ pontos críticos de $\left.I_{\lambda}\right|_{M_{\lambda}}$. Pelo Corolário 3.8 , estes são pontos críticos de $I_{\lambda}$.

\subsection{Provas dos Teorema 3.2 e Corolário 3.3}

Nesta seção usaremos os resultados mostrados na Seção A.3 do Apêndice A. Verifiquemos, então, que as condições exigidas na referida seção são satisfeitas. De fato:

- a condição $(i)$ segue da forma como o funcional $I_{\lambda}$ foi definido e das hipóteses sobre $f$;

- $(i i)$ segue da Proposição 3.5 e de $H_{I_{\lambda}}(u): H_{A_{\lambda}}^{1}\left(\Omega_{\lambda}, \Gamma_{0 \lambda}\right) \times H_{A_{\lambda}}^{1}\left(\Omega_{\lambda}, \Gamma_{0 \lambda}\right) \rightarrow \mathbb{R}$ dada por $H_{I_{\lambda}}(u)(v, w)=\langle v, w\rangle_{A_{\lambda}}-f(1)(q-1) \int_{\Omega_{\lambda}}|u|^{q-2} \operatorname{Re}(w \bar{v}) \mathrm{d} x, \quad \forall v, w \in H_{A_{\lambda}}^{1}\left(\Omega_{\lambda}, \Gamma_{0 \lambda}\right)$, ser uma forma bilinear simétrica, para todo $u \in H_{A_{\lambda}}^{1}\left(\Omega_{\lambda}, \Gamma_{0 \lambda}\right)$;

- $\left(\right.$ iii) e (iv) são consequências da Proposição 3.6 - vale ressaltar que $\delta_{0}>0$ independente de $\lambda>0$;

- $(v)$ segue pelo Lema 3.16 para $\lambda>\lambda^{*}$ : temos $\Phi_{\lambda}: \Gamma_{1 \lambda}^{-} \rightarrow M_{\lambda}^{b_{\lambda}^{*}}$ e $\beta_{\lambda}: M_{\lambda}^{b_{\lambda}^{*}} \rightarrow \Gamma_{1 \lambda}^{+}$, onde $b_{\lambda}^{*}$, valor regular de $I_{\lambda}$, é dado em (3.65), e, por construção $\beta_{\lambda} \circ \Phi_{\lambda}=I d_{\Gamma_{1 \lambda}^{-}}, \Gamma_{1 \lambda}^{+}, \Gamma_{1 \lambda}^{-} \mathrm{e}$ $\Gamma_{1 \lambda}$ homotopicamente equivalentes. 
Assim, tomando, para $\lambda>\lambda^{*}$, os entes matemáticos deste capítulo que correspondem aso que foram fixados na Seção A.3 do Apêndice A, vamos às demonstrações dos resultados principais.

\section{Demonstração do Teorema 3.2.}

Seja $\lambda \geq \lambda^{*}, \lambda^{*}>0$ dado no Lema 3.16. Seja também $\mathcal{K}$ o conjunto dos pontos críticos de $I_{\lambda}$. Suponha que $\mathcal{K}$ é discreto.

Definamos

$$
\mathcal{C}_{1}:=\left\{u \in \mathcal{K} ; \delta<I_{\lambda}(u) \leq b_{\lambda}^{*}\right\}
$$

e

$$
\mathcal{C}_{2}:=\left\{u \in \mathcal{K} ; b_{\lambda}^{*}<I_{\lambda}(u)\right\}
$$

$\operatorname{com} \delta \in\left(0, \delta_{0}\right)$ dado na Proposição 3.6, $b_{\lambda}^{*}$ como em (3.65). Então, como $\mathcal{C}_{1}, \mathcal{C}_{2}$ são conjuntos críticos isolados e $\mathcal{K}=\mathcal{C}_{1} \cup \mathcal{C}_{2}$, pelas propriedades do índice de Morse generalizado

$$
i_{t}\left(\mathcal{K}, I_{\lambda}\right)=i_{t}\left(\mathcal{C}_{1},\left.I_{\lambda}\right|_{\overline{\Sigma\left(\delta, b_{\lambda}^{*}\right)}}\right)+i_{t}\left(\mathcal{C}_{2},\left.I_{\lambda}\right|_{\overline{\Sigma\left(b_{\lambda}^{*},+\infty\right)}}\right)=\sum_{u \in \mathcal{C}_{1}} i_{t}(u)+\sum_{u \in \mathcal{C}_{2}} i_{t}(u)
$$

Pelo Lema A.26,

$$
\sum_{u \in \mathcal{C}_{1}} i_{t}(u)=t \mathcal{P}_{t}\left(\Gamma_{1 \lambda}\right)+t \mathcal{Q}(t)+(1+t) \mathcal{Q}_{1}(t)
$$

e

$$
\sum_{u \in \mathcal{C}_{2}} i_{t}(u)=t^{2}\left[\mathcal{P}_{t}\left(\Gamma_{1 \lambda}\right)+\mathcal{Q}(t)-1\right]+(1+t) \mathcal{Q}_{2}(t)
$$

Então, como $\mathcal{Q}$ é um polinômio com coeficientes não negativos,

$$
\sum_{u \in \mathcal{K}} i_{t}(u)=t \mathcal{P}_{t}\left(\Gamma_{1 \lambda}\right)+t^{2}\left[\mathcal{P}_{t}\left(\Gamma_{1 \lambda}\right)-1\right]+(1+t) \mathcal{Q}_{3}(t)
$$

onde $\mathcal{Q}_{3}$ é um polinômio com coeficientes não negativos.

\section{Demonstração do Corolário 3.3.}

Seja $\lambda \geq \lambda^{*}, \lambda^{*}>0$ dado na Proposição 3.16. Seja também $\mathcal{K}$ o conjunto dos pontos críticos de $I_{\lambda}$. Suponha que todos os pontos críticos de $I_{\lambda}$ são não degenerados. Basta, então, observar que, nestas circunstâncias,

$$
i(u)=t^{m(u)} \text {, para todo } u \in \mathcal{K} .
$$




$\frac{10}{4}$

\section{Multiplicidade de soluções não-triviais para equações de Schrödinger não-lineares com presença de campo magnético externo e condição de fronteira mista: caso geral}

Neste capítulo, aplicaremos a Teoria de Morse para obtermos multiplicidade de soluções do problema

$$
\left\{\begin{aligned}
\left(-i \nabla-A_{\lambda}\right)^{2} u+u & =f\left(|u|^{2}\right) u, \text { em } \Omega_{\lambda} \\
u & =0, \text { sobre } \Gamma_{0 \lambda} \\
\frac{\partial u}{\partial \nu} & =0, \text { sobre } \Gamma_{1 \lambda}
\end{aligned}\right.
$$

onde $i$ é a unidade imaginária, $\lambda$ é um parâmetro positivo, $\Omega_{\lambda}:=\lambda \Omega$ um domínio em expansão, $\Omega \subset \mathbb{R}^{N}, N \geq 3$, é um domínio limitado, com fronteira suave $\partial \Omega=\bar{\Gamma}_{0} \cup \bar{\Gamma}_{1}$, onde $\Gamma_{0}, \Gamma_{1}$ são subvariedades suaves disjuntas, a medida de Lebesgue $(N-1)$-dimensional de $\Gamma_{1}$ 
é positiva em $\mathbb{R}^{N}, \Gamma_{0 \lambda}:=\lambda \Gamma_{0}, \Gamma_{1 \lambda}:=\lambda \Gamma_{1}, A \in C(\Omega, \mathbb{R}), A_{\lambda}():.=A(\dot{\bar{\lambda}})$ e $f \in C^{1}\left(\mathbb{R}_{+}\right)$ satisfaz:

$\left(f_{1}\right) f(s)=o(1)$ e $f^{\prime}(s)=o(1 / s)$ quando $s \rightarrow 0^{+} ;$

$\left(f_{2}\right)$ existe $q \in\left(2,2^{*}\right)$ tal que

$$
\lim _{s \rightarrow \infty} \frac{f(s)}{s^{\frac{q-2}{2}}}=0 \text { e } \lim _{s \rightarrow \infty} \frac{f^{\prime}(s)}{s^{\frac{q-4}{2}}}=0
$$

onde $2^{*}=\frac{2 N}{N-2}$

$\left(f_{3}\right)$ existe $\theta>2$ tal que

$$
0<\frac{\theta}{2} F(s) \leq s f(s), \text { para } s>0
$$

onde $F(s)=\int_{0}^{s} f(t) \mathrm{d} t$

$\left(f_{4}\right) f^{\prime}(s)>0$, para todo $s>0$;

$\left(f_{5}\right)$ existem $q \in\left(2,2^{*}\right)$ e uma constante $C>0$ tal que

$$
s f(s)-F(s) \geq C|s|^{\frac{q}{2}}, \text { para todo } s \geq 0 .
$$

Afirmamos que o campo magnético não afeta o número de solução de $\left(P_{A, \kappa, p}\right)$. Mais especificamente, nossos resultados principais são os seguintes:

Teorema 4.1. Existe $\lambda^{*}>0$ tal que, para $\lambda>\lambda^{*}$, o problema $\left(P_{A_{\lambda}}\right)$ possui, pelo menos, $\operatorname{cat}\left(\Gamma_{1 \lambda}\right)$ soluções.

Teorema 4.2. Seja $\lambda^{*}>0$ dado no Teorema 4.1. Se $\lambda>\lambda^{*}$ e o conjunto $\mathcal{K}$ de soluções de $\left(P_{A_{\lambda}}\right)$ é discreto, então

$$
\sum_{u \in \mathcal{K}} i_{t}(u)=t \mathcal{P}_{t}\left(\Gamma_{1 \lambda}\right)+t^{2}\left[\mathcal{P}_{t}\left(\Gamma_{1 \lambda}\right)-1\right]+(t+1) \mathcal{Q}(t)
$$

onde $\mathcal{Q}$ é um polinômio de coeficientes inteiros não-negativos.

No caso em que as soluções de $\left(P_{A_{\lambda}}\right)$ são não-degeneradas, temos o

Corolário 4.3. Seja $\lambda^{*}>0$ dado no Teorema 4.1. Se $\lambda>\lambda^{*}$ e as soluções de $\left(P_{A_{\lambda}}\right)$ são não-degeneradas, então

$$
\sum_{u \in \mathcal{K}} t^{\mu(u)}=t \mathcal{P}_{t}\left(\Gamma_{1 \lambda}\right)+t^{2}\left[\mathcal{P}_{t}\left(\Gamma_{1 \lambda}\right)-1\right]+(t+1) \mathcal{Q}(t)
$$

onde $\mathcal{Q}$ é um polinômio de coeficientes inteiros não-negativos. 
Uma vez que o caso homogêneo $f(s)=f(1) s^{\frac{q-2}{2}}$ tratado no Capítulo 3 é um caso particular do caso geral, o método desenvolvido neste capítulo pode ser aplicado ao problema anterior para obtermos as mesmas conclusões para os Teoremas 3.1 e 3.2. A principal diferença entre os argumentos empregados aqui em relação aos usados no Capítulo 3 está no fato de o caso homogêneo permitir o uso de minimização em esferas de $L^{q}$ e, desta forma, estabelecer resultados de concentração como no Lema 3.14 via Lema de Concentração-Compacidade de Lions, enquanto que no presente capítulo concluímos um outro tipo de concentração, como podemos ver na Afirmação II da Proposição 4.13, cuja conclusão envolveu um estudo mais delicado.

\subsection{Condição (PS)}

Para cada $\lambda>0$, definamos o espaço de Hilbert

$$
H_{A_{\lambda}}^{1}\left(\Omega_{\lambda}, \Gamma_{0 \lambda}\right):=\left\{u \in L^{2}\left(\Omega_{\lambda}, \mathbb{C}\right) ;\left|\nabla_{A_{\lambda}} u\right| \in L^{2}\left(\Omega_{\lambda}\right), \operatorname{traço}(u)=0 \text { em } \Gamma_{0 \lambda}\right\},
$$

onde

$$
\nabla_{A_{\lambda}} u:=\left(D_{A_{\lambda}}^{j} u\right)_{j=1}^{N}, \quad D_{A_{\lambda}}^{j} u:=-i \partial_{j} u-A_{\lambda}^{j} u
$$

munido do produto interno

$$
\langle u, v\rangle_{A_{\lambda}}:=\operatorname{Re}\left\{\int_{\Omega_{\lambda}} \nabla_{A_{\lambda}} u \overline{\nabla_{A_{\lambda}} v}+u \bar{v} \mathrm{~d} x\right\}
$$

onde $\operatorname{Re}(w)$ é a parte real de $w \in \mathbb{C}$ e $\bar{w}$ é o seu conjugado complexo. A norma induzida por este produto interno é dada por

$$
\|u\|_{A_{\lambda}}:=\left(\int_{\Omega_{\lambda}}\left(\left|\nabla_{A_{\lambda}} u\right|^{2}+|u|^{2}\right) \mathrm{d} x\right)^{2}
$$

Assim como no Capítulo 3, também valem a desigualdade diamagnética e suas consequências (ver Lema 3.4). Além disso, ainda temos a condição (3.1).

Para cada $\lambda>0$, o funcional energia associado ao problema $\left(P_{A_{\lambda}}\right) I_{\lambda}: H_{A_{\lambda}}^{1}\left(\Omega_{\lambda}, \Gamma_{0 \lambda}\right) \rightarrow \mathbb{R}$ é dado por

$$
I_{\lambda}(u):=\frac{1}{2} \int_{\Omega_{\lambda}}\left(\left|\nabla_{A_{\lambda}} u\right|^{2}+|u|^{2}\right) \mathrm{d} x-\frac{1}{2} \int_{\Omega_{\lambda}} F\left(|u|^{2}\right) \mathrm{d} x
$$

Por $\left(f_{1}\right)-\left(f_{2}\right), I_{\lambda}$ está bem definido e é de classe $C^{2}\left(H_{A_{\lambda}}^{1}\left(\Omega_{\lambda}, \Gamma_{0 \lambda}\right), \mathbb{R}\right)$. Além disso

$$
I_{\lambda}^{\prime}(u) v:=\operatorname{Re}\left\{\int_{\Omega_{\lambda}}\left(\nabla_{A_{\lambda}} u \overline{\nabla_{A_{\lambda}} v}+u \bar{v}\right) \mathrm{d} x-\int_{\Omega_{\lambda}} f\left(|u|^{2}\right) u \bar{v} \mathrm{~d} x\right\},
$$

para todo $u, v \in H_{A_{\lambda}}^{1}\left(\Omega_{\lambda}, \Gamma_{0 \lambda}\right)$. Assim, todo ponto crítico de $I_{\lambda}$ é uma solução fraca de $\left(P_{A_{\lambda}}\right)$.

Vale ressaltar que, como consequência de $\left(f_{1}\right)-\left(f_{3}\right)$, temos: 
$\left(f_{6}\right)$ dado $\epsilon>0$, existe $C_{\epsilon}>0$ tal que

$$
f(s) \leq \epsilon+C_{\epsilon}|s|^{\frac{q-2}{2}}, \quad \forall s \geq 0
$$

$\left(f_{7}\right)$ existe constante $C>0$ tal que

$$
F(s) \geq C|s|^{\frac{\theta}{2}}-C, \forall s \geq 0
$$

e, consequentemente, $\lim _{s \rightarrow \infty} f\left(s^{2}\right)=+\infty$.

Proposição 4.4. O funcional $I_{\lambda}$ satisfaz a condição de Palais-Smale, isto é, toda sequência $\left(u_{n}\right) \subset H_{A_{\lambda}}^{1}\left(\Omega_{\lambda}, \Gamma_{0 \lambda}\right)$ com $\sup _{n \in \mathbb{N}}\left|I_{\lambda}\left(u_{n}\right)\right|<+\infty$ e $I_{\lambda}^{\prime}\left(u_{n}\right) \rightarrow 0$ quando $n \rightarrow \infty$, possui uma subsequência convergente.

\section{Demonstração.}

Análoga à da Proposição 3.5, lembrando que, por $\left(f_{6}\right)$,

$$
\sup _{n}\left|f\left(\left|u_{n}\right|^{2}\right) u_{n}-f\left(|u|^{2}\right) u\right|_{\frac{q}{q-1}, \Omega_{\lambda}}<\infty
$$

Tendo em vista $\left(f_{6}\right)$ e $\left(f_{7}\right)$, o funcional $I_{\lambda}$ possui a geometria do passo da montanha. Dessa forma, pela Proposição 4.4 e pelo Teorema do Passo da Montanha de Ambrosetti-Rabinowitz, para cada $\lambda>0,\left(P_{A_{\lambda}}\right)$ possui pelo menos uma solução não-trivial $u_{\lambda} \in H_{A_{\lambda}}^{1}\left(\Omega_{\lambda}, \Gamma_{0 \lambda}\right)$ tal que $I_{\lambda}\left(u_{\lambda}\right)=b_{\lambda}$ e $I_{\lambda}^{\prime}\left(u_{\lambda}\right)=0$, onde $b_{\lambda}$ denota o nível do passo da montanha de $I_{\lambda}$. Por $\left(f_{4}\right)$, argumentando como em [43, Teorema 4.2], temos

$$
b_{\lambda}:=\inf _{u \in M_{\lambda}} I_{\lambda}(u)
$$

com

$$
M_{\lambda}=\left\{u \in H_{A_{\lambda}}^{1}\left(\Omega_{\lambda}, \Gamma_{0 \lambda}\right) \backslash\{0\} ; I_{1, \Omega_{\lambda}}^{\prime}(u) u=0\right\},
$$

a variedade Nehari de $I_{\lambda}$. Sobre o funcional $I_{\lambda}$ e a variedade $M_{\lambda}$ temos os seguintes resultados.

Proposição 4.5. A variedade $M_{\lambda}$ é difeomorfa à esfera unitária em $H_{A_{\lambda}}^{1}\left(\Omega_{\lambda}, \Gamma_{0 \lambda}\right)$. Além disso, existe $\delta_{0}>0$ independente de $\lambda>0$ tal que, para todo $u \in M_{\lambda}$,

$$
\|u\|_{A_{\lambda}} \geq \delta_{0} \quad \text { e } \quad I_{\lambda}(u) \geq \delta_{0} .
$$

A demonstração da Proposição 4.5 segue os mesmos passos da da Proposição 1.7, Capítulo 1 , com o auxílio de $\left(f_{3}\right)$ e $\left(f_{6}\right)$.

Proposição 4.6. O funcional $\left.I_{\lambda}\right|_{M_{\lambda}}$ satisfaz a condição de Palais-Smale. 


\section{Demonstração.}

Seja $\left(u_{n}\right) \in M_{\lambda}$ uma sequência tal que $\sup _{n}\left|I_{\lambda}\left(u_{n}\right)\right|<\infty$ e $\left(\left.I_{\lambda}\right|_{M_{\lambda}}\right)^{\prime}\left(u_{n}\right) \rightarrow 0$. Podemos supor, tomando uma subsequência se necessário, que $I_{\lambda}\left(u_{n}\right) \rightarrow d$, para algum $d \in \mathbb{R}$. Como em [43, Proposição 5.12], existe $\mu_{n} \in \mathbb{R}$, para todo $n \in \mathbb{N}$, tal que

$$
I_{\lambda}^{\prime}\left(u_{n}\right)-\mu_{n} G_{\lambda}^{\prime}\left(u_{n}\right)=\left(\left.I_{\lambda}\right|_{M_{\lambda}}\right)^{\prime}\left(u_{n}\right)=o_{n}(1), \quad \forall n \in \mathbb{N},
$$

onde

$$
G_{\lambda}(v)=I_{\lambda}^{\prime}(v) v, \quad \forall v \in H_{A_{\lambda}}^{1}\left(\Omega_{\lambda}, \Gamma_{0 \lambda}\right) .
$$

Como na Proposição $3.5,\left(u_{n}\right)$ é limitada.

Como $u_{n} \in M_{\lambda}$, temos

$$
\begin{aligned}
G_{\lambda}^{\prime}\left(u_{n}\right) u_{n} & =2 \int_{\Omega_{\lambda}}\left|\nabla_{A_{\lambda}} u_{n}\right|^{2}+\left|u_{n}\right|^{2}-f^{\prime}\left(\left|u_{n}\right|^{2}\right)\left|u_{n}\right|^{2}-f^{\prime}\left(\left|u_{n}\right|^{2}\right)\left|u_{n}\right|^{4} \\
& =-2 \int_{\Omega_{\lambda}} f^{\prime}\left(\left|u_{n}\right|^{2}\right)\left|u_{n}\right|^{4} .
\end{aligned}
$$

Além disso, pela Proposição 4.5, existe $\delta_{0}>0$ tal que

$$
\left\|u_{n}\right\|_{A_{\lambda}} \geq \delta_{0}, \quad \forall n \in \mathbb{N}
$$

ou seja, como $u_{n} \in M_{\lambda}$,

$$
\int_{\Omega_{\lambda}} f\left(\left|u_{n}\right|^{2}\right)\left|u_{n}\right|^{2} \geq \delta_{0}, \quad \forall n \in \mathbb{N} .
$$

Por (4.3) e $\left(f_{6}\right)$, segue do Lema de Lions (ver [43], Lema 1.21) que existem $\left(y_{n}\right) \subset \Omega_{\lambda}, l>0$ e $\gamma>0$ tais que

$$
\liminf _{n \rightarrow \infty} \int_{\Omega_{\lambda} \cap B_{l}\left(y_{n}\right)}\left|u_{n}\right|^{p} \geq \gamma>0, \quad \forall p \in\left[1,2^{*}\right) .
$$

Notemos que, a menos de subsequência, $y_{n} \rightarrow y \in \overline{\Omega_{\lambda}}$. Assim, aumentando $l$ se necessário, podemos reescrever (4.4) como

$$
\liminf _{n \rightarrow \infty} \int_{\Omega_{\lambda} \cap B_{l}(y)}\left|u_{n}\right|^{p} \geq \gamma>0, \quad \forall p \in\left[1,2^{*}\right) .
$$

Seja, para cada $n \in \mathbb{N}$,

$$
\tilde{u}_{n}(x):=u_{n}(x+y), \quad \forall x \in \Omega_{\lambda}-y .
$$

Então $\left(\tilde{u}_{n}\right) \subset H_{A_{\lambda}(.+y)}^{1}\left(\Omega_{\lambda}-y, \Gamma_{0 \lambda}-y\right)$ é uma sequência limitada, uma vez que $\left(u_{n}\right)$ o é, e existe $\tilde{u} \in H_{A_{\lambda}(+y)}^{1}\left(\Omega_{\lambda}-y, \Gamma_{0 \lambda}-y\right)$ tal que valem, a menos de subsequência, os limites

$$
\left\{\begin{array}{l}
\tilde{u}_{n} \rightarrow \tilde{u} \text { em } H_{A_{\lambda}(.+y)}^{1}\left(\Omega_{\lambda}-y, \Gamma_{0 \lambda}-y\right) \\
\tilde{u}_{n} \rightarrow \tilde{u} \text { em } L^{p}\left(\Omega_{\lambda}-y, \mathbb{C}\right), \forall p \in\left[1,2^{*}\right) \\
\tilde{u}_{n} \rightarrow \tilde{u} \text { quase sempre em } \Omega_{\lambda}-y
\end{array}\right.
$$


quando $n \rightarrow \infty$. Portanto, por (4.5),

$$
\int_{\Omega_{\lambda}-y}|\tilde{u}|^{2} \geq \gamma>0
$$

isto é, $\tilde{u} \not \equiv 0$.

Agora, por (4.2), (4.6) e $\left(f_{4}\right)$, aplicando o Lema de Fatou,

$$
\begin{aligned}
\liminf _{n \rightarrow \infty} G_{\lambda}^{\prime}\left(u_{n}\right) u_{n} & =\liminf _{n \rightarrow \infty}-2 \int_{\Omega_{\lambda}} f^{\prime}\left(\left|u_{n}\right|^{2}\right)\left|u_{n}\right|^{4} \\
& =\liminf _{n \rightarrow \infty}-2 \int_{\Omega_{\lambda}-y} f^{\prime}\left(\left|\tilde{u}_{n}\right|^{2}\right)\left|\tilde{u}_{n}\right|^{4} \\
& \leq-2 \int_{\Omega_{\lambda}-y} f^{\prime}\left(|\tilde{u}|^{2}\right)|\tilde{u}|^{4}<0 .
\end{aligned}
$$

Dessa forma, aplicando $u_{n}$ a ambos os membros de (4.1), obtemos $\mu_{n}=o_{n}(1)$. Logo $I_{\lambda}\left(u_{\lambda}\right) \rightarrow 0$ e $I_{\lambda}^{\prime}\left(u_{n}\right) \rightarrow 0$ quando $n \rightarrow \infty$. O resultado segue da Proposição 4.4 .

De forma análoga ao resultado anterior, podemos mostrar o

Corolário 4.7. Todo ponto crítico de $\left.I_{\lambda}\right|_{M_{\lambda}}$ é um ponto crítico de $I_{\lambda}$.

\subsection{Preliminares}

Nesta seção, nosso objetivo é buscar relações entre subconjuntos de $\mathbb{R}^{N}$ e do espaço de funções $H_{A_{\lambda}}^{1}\left(\Omega_{\lambda}, \Gamma_{0 \lambda}\right)$.

Sejam os problemas

$$
\left\{\begin{aligned}
-\Delta u+u & =f\left(u^{2}\right) u, \text { em } \mathbb{R}_{+}^{N}, \\
\frac{\partial u}{\partial \nu} & =0, \text { sobre } \mathbb{R}^{N-1}
\end{aligned}\right.
$$

onde $\mathbb{R}^{N-1}=\left\{\left(x_{1}, \ldots, x_{N-1}, 0\right) ; x_{i} \in \mathbb{R}, i=1, \ldots, N-1\right\}$, e

$$
\left\{\begin{array}{l}
-\Delta u+u=f\left(u^{2}\right) u, \text { em } \mathbb{R}^{N} \\
u \in H^{1}\left(\mathbb{R}^{N}\right), u>0 \text { em } \mathbb{R}^{N}
\end{array}\right.
$$

e consideremos seus respectivos funcionais energia associados

$$
J_{\infty}(u):=\frac{1}{2} \int_{\mathbb{R}_{+}^{N}}\left(|\nabla u|^{2}+u^{2}\right) \mathrm{d} x-\frac{1}{2} \int_{\mathbb{R}_{+}^{N}} F\left(u^{2}\right) \mathrm{d} x, \quad \forall u \in H^{1}\left(\mathbb{R}_{+}^{N}\right),
$$

$\mathrm{e}$

$$
J_{\mathbb{R}^{N}}(u):=\frac{1}{2} \int_{\mathbb{R}^{N}}\left(|\nabla u|^{2}+u^{2}\right) \mathrm{d} x-\frac{1}{2} \int_{\mathbb{R}^{N}} F\left(u^{2}\right) \mathrm{d} x, \quad \forall u \in H^{1}\left(\mathbb{R}^{N}\right) .
$$

Consideremos também as variedades de Nehari e níveis do passo da montanha correspondentes:

$$
N_{\infty}:=\left\{u \in H^{1}\left(\mathbb{R}_{+}^{N}\right) \backslash\{0\} ; J_{\infty}^{\prime}(u) u=0\right\}, \quad c_{\infty}:=\inf _{N_{\infty}} J_{\infty}
$$


e

$$
N_{\mathbb{R}^{N}}:=\left\{u \in H^{1}\left(\mathbb{R}^{N}\right) \backslash\{0\} ; J_{\mathbb{R}^{N}}^{\prime}(u) u=0\right\}, \quad c_{\mathbb{R}^{N}}:=\inf _{N_{\mathbb{R}^{N}}} J_{\mathbb{R}^{N}}
$$

Por [12, 35], $\left(P_{2}\right)$ possui uma solução positiva e radialmente simétrica $w \in H^{1}\left(\mathbb{R}^{N}\right) \cap C^{2}\left(\mathbb{R}^{N}\right)$. Além disso, a restrição de $w$ a $\mathbb{R}_{+}^{N}$ é solução de $\left(P_{1}\right)$. Como consequência,

$$
c_{\mathbb{R}^{N}}=2 c_{\infty}
$$

Seja $r>0$ tal que

$$
\Gamma_{1}^{+}:=\left\{x \in \mathbb{R}^{N} ; \operatorname{dist}\left(x, \Gamma_{1}\right) \leq r\right\}, \quad \Gamma_{1}^{-}:=\left\{x \in \Gamma_{1} ; \operatorname{dist}\left(x, \Gamma_{0}\right) \geq r\right\}
$$

são homotopicamente equivalentes a $\Gamma_{1}$. Seja $\eta \in C^{\infty}\left(\mathbb{R}_{+}\right)$uma função não crescente tal que $\eta=1 \mathrm{em}[0, r / 2], \eta=0 \mathrm{em}[r,+\infty), \eta^{\prime} \in L^{\infty}\left(\mathbb{R}_{+}\right)$. Denotaremos por $\Gamma_{1 \lambda}^{-}$e $\Gamma_{1 \lambda}^{+}$os conjuntos $\lambda \Gamma_{1}^{-}$e $\lambda \Gamma_{1}^{+}$, respectivamente. Para qualquer $y \in \Gamma_{1 \lambda}^{-}$, definimos a função

$$
x \in \Omega_{\lambda} \mapsto e^{i \tau_{\lambda, y}(x)} \eta\left(\frac{|x-y|}{\lambda}\right) w(x-y),
$$

onde $\tau_{\lambda, y}(x):=\sum_{j=1}^{N} A_{\lambda}^{j}(y) x^{j}$. Pela definição de $\eta$, esta função pertence a $H_{A_{\lambda}}^{1}\left(\Omega_{\lambda}, \Gamma_{0 \lambda}\right)$. Por $\left(f_{1}\right)-\left(f_{4}\right)$, existe $t_{\lambda, y}>0$ tal que

$$
t_{\lambda, y} e^{i \tau_{\lambda, y}} \eta\left(\frac{|.-y|}{\lambda}\right) w(.-y) \in M_{\lambda} .
$$

Definamos, portanto, $\Phi_{\lambda}: \Gamma_{1 \lambda}^{-} \rightarrow M_{\lambda}$ como

$$
\Phi_{\lambda}(y)(x)=t_{\lambda, y} e^{i \tau_{\lambda, y}(x)} \eta\left(\frac{|x-y|}{\lambda}\right) w(x-y), \quad \forall x \in \Omega_{\lambda}, y \in \Gamma_{1 \lambda}^{-} .
$$

As demonstrações dos próximos resultados são idênticas às dos Lemas 3.9 e 3.10 do Capítulo 3 .

Lema 4.8. Vale

$$
\lim _{\lambda \rightarrow \infty} \max _{y \in \Gamma_{1 \lambda}^{-}}\left|I_{\lambda}\left(\Phi_{\lambda}(y)\right)-c_{\infty}\right|=0
$$

Lema 4.9. Sejam $l>0$ e $2 \leq p<2^{*}$. Se a família $\left\{u_{\lambda} \in H^{1}\left(\Omega_{\lambda}\right)\right\}_{\lambda>0}$ é limitada uniformemente em $\lambda>0$ e satisfaz

$$
\lim _{\lambda \rightarrow \infty} \sup _{y \in \mathbb{R}^{N}} \int_{B_{l}(y) \cap \Omega_{\lambda}}\left|u_{\lambda}\right|^{p}=0,
$$

então

$$
\lim _{\lambda \rightarrow \infty} \int_{\Omega_{\lambda}}\left|u_{\lambda}\right|^{m}=0, \quad \forall m \in\left(2,2^{*}\right) .
$$




\subsection{Comportamento dos níveis minimax}

A demonstração da próxima proposição é idêntica à da Proposição 3.11. Porém, por uma questão de completude e para facilitar a leitura dos próximos resultados, fa-la-emos.

Proposição 4.10. Temos

$$
\lim _{\lambda \rightarrow \infty} b_{\lambda}=c_{\infty}
$$

\section{Demonstração.}

Primeiramente observemos que pela definição de $\Phi_{\lambda}(y)$ e o Lema 4.8,

$$
b_{\lambda} \leq I_{\lambda}\left(\Phi_{\lambda}(y)\right) \leq o_{\lambda}(1)+c_{\infty} .
$$

Consideremos os problemas auxiliares

$$
\left\{\begin{aligned}
-\Delta u+u & =f\left(u^{2}\right) u, \text { em } \Omega_{\lambda} \\
\frac{\partial u}{\partial \nu} & =0, \text { sobre } \Gamma_{1 \lambda}, \\
u & =0, \text { sobre } \Gamma_{0 \lambda}
\end{aligned}\right.
$$

e

$$
\left\{\begin{aligned}
-\Delta u+u & =f\left(u^{2}\right) u, \text { em } \Omega_{\lambda}, \\
\frac{\partial u}{\partial \nu} & =0, \text { sobre } \partial \Omega_{\lambda} .
\end{aligned}\right.
$$

Denotaremos por $H^{1}\left(\Omega_{\lambda}, \Gamma_{0 \lambda}\right)$ o espaço de Hilbert

$$
H^{1}\left(\Omega_{\lambda}, \Gamma_{0 \lambda}\right):=\left\{u \in H^{1}\left(\Omega_{\lambda}\right) ; \operatorname{traço}(u)=0 \text { em } \Gamma_{0 \lambda}\right\}
$$

munido da norma usual de $H^{1}\left(\Omega_{\lambda}\right)$, a qual denotaremos por $\|\cdot\|_{\Omega_{\lambda}}$. Sejam $J_{\lambda}: H^{1}\left(\Omega_{\lambda}, \Gamma_{0 \lambda}\right) \rightarrow \mathbb{R}$, o funcional energia associado ao problema $\left(P_{3}\right)$, dado por

$$
J_{\lambda}(u)=\frac{1}{2} \int_{\Omega_{\lambda}}\left(|\nabla u|^{2}+u^{2}\right) \mathrm{d} x-\frac{1}{2} \int_{\Omega_{\lambda}} F\left(u^{2}\right) \mathrm{d} x, \quad \forall u \in H^{1}\left(\Omega_{\lambda}, \Gamma_{0 \lambda}\right) .
$$

e $c_{\lambda}$ o nível do passo da montanha associado. Definimos também $\bar{J}_{\lambda}: H^{1}\left(\Omega_{\lambda}\right) \rightarrow \mathbb{R}$, o funcional energia associado ao problema $\left(P_{4}\right)$, por

$$
\bar{J}_{\lambda}(u)=\frac{1}{2} \int_{\Omega_{\lambda}}\left(|\nabla u|^{2}+u^{2}\right) \mathrm{d} x-\frac{1}{2} \int_{\Omega_{\lambda}} F\left(u^{2}\right) \mathrm{d} x, \quad \forall u \in H^{1}\left(\Omega_{\lambda}\right)
$$

com variedade de Nehari e nível do passo da montanha correspondentes

$$
\bar{N}_{\lambda}:=\left\{u \in H^{1}\left(\Omega_{\lambda}\right) \backslash\{0\} ; \bar{J}^{\prime}(u) u=0\right\} \quad \text { e } \quad \bar{c}_{\lambda}:=\inf _{\bar{N}_{\lambda}} \bar{J}_{\lambda} .
$$

Pela definição dos níveis dados anteriormente e pela desigualdade diamagnética, temos

$$
b_{\lambda} \geq c_{\lambda} \geq \bar{c}_{\lambda}>0
$$


Por (4.8) e (4.9), é suficiente mostrarmos que

$$
\lim _{\lambda \rightarrow \infty} \bar{c}_{\lambda}=c_{\infty}
$$

Para provarmos o limite (4.10), comecemos observando que, argumentando como na Proposição 3.5 e usando o Teorema do Passo da Montanha, existe $u_{\lambda} \in H^{1}\left(\Omega_{\lambda}\right)$ tal que

$$
\bar{J}_{\lambda}\left(u_{\lambda}\right)=\bar{c}_{\lambda}=\inf _{\bar{N}_{\lambda}} \bar{J}_{\lambda}, \quad \bar{J}_{\lambda}^{\prime}\left(u_{\lambda}\right)=0,
$$

para cada $\lambda>0$. Por $(4.9)$ e $(4.11),\left(\bar{J}_{\lambda}\left(u_{\lambda}\right)\right)_{\lambda}$ é limitada e $\bar{J}_{\lambda}^{\prime}\left(u_{\lambda}\right) u_{\lambda}=0$, para todo $\lambda>0$. Daí, por $\left(f_{3}\right)$,

$$
\sup _{\lambda>0}\left\|u_{\lambda}\right\|_{\Omega_{\lambda}}<+\infty
$$

Procedendo como na demonstração da Proposição 3.6, podemos supor, sem perda de generalidade, que

$$
\left\|u_{\lambda}\right\|_{\Omega_{\lambda}}^{2} \geq \delta_{0} \quad \text { e } \quad \bar{J}_{\lambda}\left(u_{\lambda}\right) \geq \bar{c}_{\lambda} \geq \delta_{0}, \quad \forall \lambda>0
$$

onde, como antes, $\delta_{0}>0$ independe de $\lambda$. Portanto, por (4.13) e pelo Lema 4.9, existem $\left(y_{\lambda}\right)_{\lambda} \subset \mathbb{R}^{N}, l>0$ e $\gamma>0$ tais que

$$
\liminf _{\lambda \rightarrow \infty} \int_{\Omega_{\lambda} \cap B_{l}\left(y_{\lambda}\right)}\left|u_{\lambda}\right|^{2} \geq \gamma>0 .
$$

Mais ainda, aumentando $l$ se necessário, podemos supor que $y_{\lambda} \in \Omega_{\lambda}$, para todo $\lambda>0$, uma vez que (4.14) implica que $\Omega_{\lambda} \cap B_{l}\left(y_{\lambda}\right) \neq 0$, para todo $\lambda$.

Lema 4.11. Existe uma constante $C>0$ tal que $\operatorname{dist}\left(y_{\lambda}, \partial \Omega_{\lambda}\right) \leq C$.

\section{Demonstração.}

Suponha, por contradição, que existe $\lambda_{n} \rightarrow+\infty$ tal que $\operatorname{dist}\left(y_{\lambda_{n}}, \partial \Omega_{\lambda_{n}}\right) \rightarrow \infty$, quando $n \rightarrow \infty$.

Seja $R>l$ arbitrário. Para $n$ suficientemente grande, $B_{2 R}\left(y_{\lambda_{n}}\right) \subset \Omega_{\lambda_{n}}$. Definamos

$$
w_{\lambda_{n}, R}(x):=\eta\left(\frac{|x|}{R}\right) u_{\lambda_{n}}\left(x+y_{\lambda_{n}}\right), \quad \forall x \in \Omega_{\lambda_{n}}-y_{\lambda_{n}},
$$

onde $\eta \in C^{\infty}\left(\mathbb{R}_{+}\right)$é tal que $\eta=1 \mathrm{em}[0,1], \eta=0$ em $(2,+\infty), \eta^{\prime} \in L^{\infty}\left(\mathbb{R}_{+}\right)$. Então $\operatorname{supp} w_{\lambda_{n}, R} \subset B_{2 R}(0)$. Sem perda de generalidade, podemos supor que $w_{\lambda_{n}, R} \in H^{1}\left(\mathbb{R}^{N}\right)$, estendendo esta aplicação por zero fora de $\Omega_{\lambda_{n}}-y_{\lambda_{n}}$. Mais ainda, por construção, $\sup _{n}\left\|w_{\lambda_{n}, R}\right\| \leq C$, independente de $R$. Além disso, como

$$
\int_{B_{l}(0)}\left|w_{\lambda_{n}, R}\right|^{2} \mathrm{~d} x=\int_{B_{l}(0)}\left|u_{\lambda_{n}}\left(x+y_{\lambda_{n}}\right)\right|^{2} \mathrm{~d} x=\int_{B_{l}\left(y_{\lambda_{n}}\right)}\left|u_{\lambda_{n}}\right|^{2} \mathrm{~d} x \geq \gamma>0,
$$

existe $w_{R} \in H^{1}\left(\mathbb{R}^{N}\right) \backslash\{0\}$ tal que

$$
\left\{\begin{array}{l}
w_{\lambda, R} \rightarrow w_{R} \text { em } H^{1}\left(\mathbb{R}^{N}\right), \text { quando } n \rightarrow \infty, \\
w_{\lambda, R} \rightarrow w_{R}, \text { em } L_{l o c}^{p}\left(\mathbb{R}^{N}\right), p \in\left[1,2^{*}\right), \text { quando } n \rightarrow \infty \\
\int_{B_{l}(0)}\left|w_{R}\right|^{2} \mathrm{~d} x \geq \gamma>0 .
\end{array}\right.
$$


Já que $\left\|w_{R}\right\| \leq \liminf _{n \rightarrow \infty}\left\|w_{\lambda_{n}, R}\right\|$, a família $\left(w_{R}\right)_{R} \subset H^{1}\left(\mathbb{R}^{N}\right)$ é limitada. Assim, existe $v \in H^{1}\left(\mathbb{R}^{N}\right)$ tal que

$$
\left\{\begin{array}{l}
w_{R} \rightarrow v \text { em } H^{1}\left(\mathbb{R}^{N}\right), \text { quando } R \rightarrow \infty, \\
w_{R} \rightarrow v, \text { em } L_{l o c}^{p}\left(\mathbb{R}^{N}\right), p \in\left[1,2^{*}\right), \text { quando } R \rightarrow \infty, \\
\int_{B_{l}(0)}|v|^{2} \mathrm{~d} x \geq \gamma>0 .
\end{array}\right.
$$

Em particular, $v \not \equiv 0$. Mostremos que $v$ é uma solução de $\left(P_{2}\right)$. De fato, dada $\phi \in C_{c}^{\infty}\left(\mathbb{R}^{N}\right)$, tomemos $t>0$ tal que supp $\phi \subset B_{t}(0)$. Para $n$ suficientemente grande, $B_{t}\left(y_{\lambda_{n}}\right) \subset \Omega$. Como $u_{\lambda_{n}}$ é solução de $\left(P_{4}\right)$ para $\lambda_{n}=\lambda$, temos

$$
\begin{aligned}
\int_{B_{t}(0)}\left[\nabla u_{\lambda_{n}}\left(x+y_{\lambda_{n}}\right) \nabla \phi+u_{\lambda_{n}}\left(x+y_{\lambda_{n}}\right) \phi\right] & =\int_{\Omega_{\lambda_{n}}}\left[\nabla u_{\lambda_{n}} \nabla \phi\left(x-y_{\lambda_{n}}\right)+u_{\lambda_{n}} \phi\left(x-y_{\lambda_{n}}\right)\right] \\
& =\int_{\Omega_{\lambda_{n}}} f\left(u_{\lambda_{n}}^{2}\right) u_{\lambda_{n}} \phi\left(x-y_{\lambda_{n}}\right) \\
& =\int_{B_{t}(0)} f\left(u_{\lambda_{n}}^{2}\left(x+y_{\lambda_{n}}\right)\right) u_{\lambda_{n}}\left(x+y_{\lambda_{n}}\right) \phi .
\end{aligned}
$$

Para $n$ suficientemente grande e $R>t$, obtemos

$$
\int_{B_{t}(0)}\left[\nabla w_{\lambda_{n}, R} \nabla \phi+w_{\lambda_{n}, R} \phi\right] \mathrm{d} x=\int_{B_{t}(0)} f\left(w_{\lambda_{n}, R}^{2}\right) w_{\lambda_{n}, R} \phi \mathrm{d} x
$$

Passando ao limite $n \rightarrow \infty$, encontramos

$$
\int_{B_{t}(0)}\left[\nabla w_{R} \nabla \phi+w_{R} \phi\right] \mathrm{d} x=\int_{B_{t}(0)} f\left(w_{R}^{2}\right) w_{R} \phi \mathrm{d} x .
$$

Lembrando que $\operatorname{supp} \phi \subset B_{t}(0)$ e $R>t$, tomando o limite $R \rightarrow \infty$, temos

$$
\int_{\mathbb{R}^{N}}[\nabla v \nabla \phi+v \phi] \mathrm{d} x=\int_{B_{t}(0)}[\nabla v \nabla \phi+v \phi] \mathrm{d} x=\int_{B_{t}(0)} f\left(v^{2}\right) v \phi \mathrm{d} x=\int_{\mathbb{R}^{N}} f\left(v^{2}\right) v \phi \mathrm{d} x .
$$

Como $\phi \in C_{c}^{\infty}$ é arbitrária, argumentando como em [43, Seção 1.6, Lema 1.30], v é solução de $\left(P_{2}\right)$.

Notemos que, para $M \leq R$ dado, $B_{M}\left(y_{\lambda_{n}}\right) \subset \Omega_{\lambda_{n}}$. Por (4.8) e (4.9),

$$
\begin{aligned}
o_{\lambda}(1)+c_{\infty} & \geq \bar{c}_{\lambda_{n}}=\bar{J}_{\lambda_{n}}\left(u_{\lambda_{n}}\right)-\frac{1}{2} \bar{J}_{\lambda_{n}}^{\prime}\left(u_{\lambda_{n}}\right) u_{\lambda_{n}} \\
& =\frac{1}{2} \int_{\Omega_{\lambda_{n}}}\left[f\left(u_{\lambda_{n}}^{2}\right) u_{\lambda_{n}}^{2}-F\left(u_{\lambda_{n}}^{2}\right) u_{\lambda_{n}}^{2}\right] \mathrm{d} x \\
& \geq \frac{1}{2} \int_{B_{M}\left(y_{\lambda_{n}}\right)}\left[f\left(u_{\lambda_{n}}^{2}\right) u_{\lambda_{n}}^{2}-F\left(u_{\lambda_{n}}^{2}\right) u_{\lambda_{n}}^{2}\right] \mathrm{d} x \\
& =\frac{1}{2} \int_{B_{M}(0)}\left[f\left(w_{\lambda_{n}, r}^{2}\right) w_{\lambda_{n}, r}^{2}-F\left(w_{\lambda_{n}, r}^{2}\right) w_{\lambda_{n}, r}^{2}\right] \mathrm{d} x .
\end{aligned}
$$

Pelo Lema de Fatou e (4.7), obtemos, depois de tomar os limites $n \rightarrow \infty, R \rightarrow \infty$ e $M \rightarrow \infty$,

$$
c_{\infty} \geq \frac{1}{2} \int_{\mathbb{R}^{N}}\left[f\left(v^{2}\right) v^{2}-F\left(v^{2}\right)\right] \mathrm{d} x=J_{\mathbb{R}^{N}}(v) \geq c_{\mathbb{R}^{N}}=2 c_{\infty},
$$


o que é um absurdo.

Pelo Lema 4.11, aumentando $l$ se necessário, podemos admitir que $y_{\lambda} \in \Omega_{\lambda}$ em (4.14).

Seja $T_{y_{\lambda}}$ a matriz de rotação em $\mathbb{R}^{N}$ tal que o vetor normal unitário interior a $\tilde{\Omega}_{\lambda}:=T_{y_{\lambda}}\left(\Omega_{\lambda}-y_{\lambda}\right)$ seja $e_{N}=(0,0, \ldots, 0,1)$. Definamos, para todo $\lambda$,

$$
v_{\lambda}(x)=u_{\lambda}\left(T_{y_{\lambda}}^{-1} x+y_{\lambda}\right), \quad \forall x \in \tilde{\Omega}_{\lambda} .
$$

Temos, sobre $v_{\lambda}$ :

(a) $\left\|v_{\lambda}\right\|_{\tilde{\Omega}_{\lambda}}=\left\|u_{\lambda}\right\|_{\Omega_{\lambda}}$, donde, por (4.12), $\sup _{\lambda>0}\left\|v_{\lambda}\right\|_{\tilde{\Omega}_{\lambda}}<+\infty$;

(b) $\int_{\tilde{\Omega}_{\lambda}} F\left(v_{\lambda}^{2}\right) \mathrm{d} x=\int_{\Omega_{\lambda}} F\left(u_{\lambda}^{2}\right) \mathrm{d} x$

(c) como $u_{\lambda}$ é solução de $\left(P_{4}\right), v_{\lambda}$ satisfaz o problema

$$
\left\{\begin{aligned}
-\Delta u+u & =f\left(u^{2}\right) u, \text { em } \tilde{\Omega}_{\lambda}, \\
\frac{\partial u}{\partial \nu} & =0, \text { sobre } \partial \tilde{\Omega}_{\lambda} ;
\end{aligned}\right.
$$

(d) $\bar{J}_{\tilde{\Omega}_{\lambda}}\left(v_{\lambda}\right)=\bar{c}_{\tilde{\Omega}_{\lambda}}=\bar{c}_{\lambda}$, onde $\bar{J}_{\tilde{\Omega}_{\lambda}}$ é o funcional energia associado ao problema $\left(P_{5}\right)$ e $\bar{c}_{\tilde{\Omega}_{\lambda}}$ o correspondente nível do passo da montanha;

(e) por (4.14),

$$
\liminf _{\lambda \rightarrow \infty} \int_{B_{l}(0) \cap \tilde{\Omega}_{\lambda}}\left|v_{\lambda}\right|^{2} \mathrm{~d} x \geq \gamma
$$

Dados $\rho>h>0$, definamos

$$
D_{\rho, h}:=\left\{x \in B_{\rho}(0) ; x^{N}>h, x=\left(x^{1}, \ldots, x^{N}\right)\right\} .
$$

Por (3.1), $\tilde{\Omega}_{\lambda} \rightarrow \mathbb{R}_{+}^{N}$ quando $\lambda \rightarrow \infty$, quase sempre em $\mathbb{R}^{N}$. Então, para $\lambda$ suficientemente grande, $D_{\rho, h} \subset \tilde{\Omega}_{\lambda}$. Logo $v_{\lambda} \in H^{1}\left(D_{\rho, h}\right)$, para $\lambda$ suficientemente grande. Por $(a)$, existe $v_{\rho, h} \in H^{1}\left(D_{\rho, h}, \mathbb{R}\right)$ tal que, a menos de subsequência,

$$
\left\{\begin{array}{l}
v_{\lambda} \rightarrow v_{\rho, h} \text { em } H^{1}\left(D_{\rho, h}, \mathbb{R}\right), \text { quando } \lambda \rightarrow \infty, \\
v_{\lambda} \rightarrow v_{\rho, h} \text { em } L^{p}\left(D_{\rho, h}, \mathbb{R}\right), p \in\left[1,2^{*}\right), \text { quando } \lambda \rightarrow \infty, \\
v_{\lambda}(x) \rightarrow v_{\rho, h}(x) \text { quase sempre em } D_{\rho, h}, \text { quando } \lambda \rightarrow \infty .
\end{array}\right.
$$

Mais ainda, novamente por $(a)$ e pelo Teorema de Banach-Steinhaus, existe uma constante $K>0$ tal que

$$
\left\|v_{\rho, h}\right\|_{D_{\rho, h}} \leq K, \quad \forall \rho, h>0 .
$$

Sejam $\rho_{n} \rightarrow \infty, h_{n} \rightarrow 0$ sequências monótonas. Então

$$
D_{n}:=D_{\rho_{n}, h_{n}} \subset D_{\rho_{n+1}, h_{n+1}}:=D_{n+1}, \quad \forall n \geq 1 .
$$


Observemos que: para $n=1$, existem $v_{1} \in H^{1}\left(D_{1}, \mathbb{R}\right)$ e subsequência $\left(v_{\lambda 1}\right) \subset\left(v_{\lambda}\right)$ tais que, quando $\lambda \rightarrow \infty$,

$$
\left\{\begin{array}{l}
v_{\lambda 1} \rightarrow v_{1} \text { em } H^{1}\left(D_{1}, \mathbb{R}\right), \\
v_{\lambda 1} \rightarrow v_{1} \text { em } L^{p}\left(D_{1}, \mathbb{R}\right), p \in\left[1,2^{*}\right) \\
v_{\lambda 1}(x) \rightarrow v_{1}(x) \text { quase sempre em } D_{1}
\end{array}\right.
$$

para $n=2$, existem $v_{2} \in H^{1}\left(D_{2}, \mathbb{R}\right)$ e subsequência $\left(v_{\lambda 2}\right) \subset\left(v_{\lambda 1}\right)$ tais que, quando $\lambda \rightarrow \infty$,

$$
\left\{\begin{array}{l}
v_{\lambda 2} \rightarrow v_{2} \text { em } H^{1}\left(D_{2}, \mathbb{R}\right) \\
v_{\lambda 2} \rightarrow v_{2} \text { em } L^{p}\left(D_{2}, \mathbb{R}\right), p \in\left[1,2^{*}\right) \\
v_{\lambda 2}(x) \rightarrow v_{2}(x) \text { quase sempre em } D_{2}
\end{array}\right.
$$

com $\left.v_{2}\right|_{D_{1}}=v_{1}$ quase sempre em $D_{1}$, pela unicidade do limite quase sempre em $D_{1}$; para $n=j$, existem $v_{j} \in H^{1}\left(D_{j}, \mathbb{R}\right)$ e subsequência $\left(v_{\lambda j}\right) \subset\left(v_{\lambda(j-1)}\right)$ tais que, quando $\lambda \rightarrow \infty$,

$$
\left\{\begin{array}{l}
v_{\lambda j} \rightarrow v_{j} \text { em } H^{1}\left(D_{j}, \mathbb{R}\right), \\
v_{\lambda j} \rightarrow v_{j} \text { em } L^{p}\left(D_{j}, \mathbb{R}\right), p \in\left[1,2^{*}\right) \\
v_{\lambda j}(x) \rightarrow v_{j}(x) \text { quase sempre em } D_{j}
\end{array}\right.
$$

com $\left.v_{j}\right|_{D_{l}}=v_{i}$ quase sempre em $D_{i}$, pela unicidade do limite quase sempre em $D_{i}$, para qualquer $i \leq j$. Seja $v_{k k} \in H^{1}\left(D_{k}, \mathbb{R}\right)$ o $k$-ésimo termo da subsequência $\left(v_{\lambda k}\right)$. Então $\left(v_{k k}\right)_{k},\left(\partial_{j} v_{k k}\right)_{k} \subset L^{2}\left(\mathbb{R}_{+}^{N}, \mathbb{R}\right)$ são sequências limitadas, donde, a menos de subsequência, convergem fraco em $L^{2}\left(\mathbb{R}_{+}^{N}, \mathbb{R}\right)$ para $v, \bar{v}_{j} \in L^{2}\left(\mathbb{R}_{+}^{N}, \mathbb{R}\right)$, respectivamente. Como $\partial_{j} v_{k k}$ é a $j$-ésima derivada parcial fraca de $v_{k k}$, temos, pela convergência fraca em $L^{2}\left(\mathbb{R}_{+}^{N}, \mathbb{R}\right)$,

$$
\partial_{j} v=\bar{v}_{j}, \forall j \in\{1, \ldots, N\}, \text { quase sempre em } \mathbb{R}^{N} .
$$

Portanto, $v \in H^{1}\left(\mathbb{R}_{+}^{N}, \mathbb{R}\right)$ e, quando $k \rightarrow \infty$,

$$
\left\{\begin{array}{l}
v_{k k} \rightarrow v \text { em } H_{l o c}^{1}\left(\mathbb{R}_{+}^{N}, \mathbb{R}\right) \\
v_{k k} \rightarrow v \text { em } L_{l o c}^{p}\left(\mathbb{R}_{+}^{N}, \mathbb{R}\right), \forall p \in\left[1,2^{*}\right) \\
v_{k k}(x) \rightarrow v(x) \text { quase sempre em } \mathbb{R}_{+}^{N}
\end{array}\right.
$$

Para simplificar a notação, ponhamos $v_{k}:=v_{k k}$ e $\tilde{\Omega}_{k}:=\tilde{\Omega}_{k k}, \forall k \in \mathbb{N}$.

Lema 4.12. A função v é uma solução não trivial de $\left(P_{1}\right)$.

\section{Demonstração.}

Primeiramente vejamos que $v \not \equiv 0$. De fato, por $(e)$,

$$
\liminf _{k \rightarrow \infty} \int_{B_{l}(0) \cap \tilde{\Omega}_{k}} v_{k}^{2} \mathrm{~d} x \geq \gamma>0 .
$$


Dado $t \in(0, l)$, definamos $A_{t}:=\left\{x \in B_{l}(0) \cap \tilde{\Omega}_{k} ; 0 \leq x^{N} \leq t\right\}$ e $\Lambda_{k}:=\left(B_{l}(0) \cap \tilde{\Omega}_{k}\right) \backslash A_{t}$. Assim

$$
\int_{B_{l}(0) \cap \tilde{\Omega}_{k}} v_{k}^{2} \mathrm{~d} x=\left(\int_{A_{t}}+\int_{B_{k}}\right) v_{k}^{2} \mathrm{~d} x .
$$

Como $\sup _{k}\left\|v_{k}\right\|_{D_{k}}<\infty$, pela desigualdade de Hölder e das imersões de Sobolev, tendo em vista o Lema 3.4, existe uma constante $\bar{K}>0$ independente de $k$ tal que

$$
\int_{A_{t}} v_{k}^{2} \mathrm{~d} x \leq\left(\int_{A_{t}} v_{k}^{2^{*}} \mathrm{~d} x\right)^{\frac{2}{2^{*}}}\left(\int_{A_{t}} \mathrm{~d} x\right)^{\frac{2}{N}} \leq \bar{K}\left|A_{t}\right|^{\frac{2}{N}} .
$$

Escolhamos $t \in(0, l)$ de modo que

$$
\int_{A_{t}} v_{k}^{2} \mathrm{~d} x \leq\left(\int_{A_{t}} v_{k}^{2^{*}} \mathrm{~d} x\right)^{\frac{2}{2^{*}}}\left(\int_{A_{t}} \mathrm{~d} x\right)^{\frac{2}{N}} \leq \bar{K}\left|A_{t}\right|^{\frac{2}{N}}<\frac{\gamma}{4} .
$$

Assim, por (4.16), para todo $k$ suficientemente grande,

$$
\frac{\gamma}{2} \leq \int_{B_{l}(0) \cap \tilde{\Omega}_{k}} v_{k}^{2} \mathrm{~d} x \leq \frac{\gamma}{4}+\int_{\Lambda_{k}} v_{k}^{2} \mathrm{~d} x \leq \frac{\gamma}{4}+\int_{D} v_{k}^{2} \mathrm{~d} x
$$

para todo conjunto compacto $D \subset \mathbb{R}^{N}$ compacto com $\Lambda_{k} \subset D \subset D_{k}$. Logo, para todo $k$ suficientemente grande,

$$
\int_{D} v_{k}^{2} \mathrm{~d} x \geq \frac{\gamma}{4}
$$

e daí, por (4.15),

$$
\int_{D} v^{2} \mathrm{~d} x=\lim _{k \rightarrow \infty} \int_{D} v_{k}^{2} \mathrm{~d} x \geq \frac{\gamma}{4}>0
$$

Portanto $v \not \equiv 0$. Para mostrarmos que $v$ é solução fraca de $\left(P_{1}\right)$, vejamos que $\nabla v_{k} \rightarrow \nabla v$ em $\left(L^{2}(K, \mathbb{R})\right)^{N}$, para qualquer $K \subset \mathbb{R}_{+}^{N}$ compacto. Com efeito, seja $K \subset \mathbb{R}_{+}^{N}$ compacto. Para $\Psi \in C_{c}^{\infty}\left(\mathbb{R}^{N}\right)$ tal que $\Psi \equiv 1$ em $K$ e $\Psi \in[0,1]$, temos $\operatorname{supp} \Psi \subset \tilde{\Omega}_{k}$, para todo $k$ suficientemente grande. Como $v_{k} \Psi, v \Psi \in H^{1}\left(\tilde{\Omega}_{k}, \mathbb{R}\right)$ e $v_{k}$ é uma solução fraca de $\left(P_{5}\right)$, temos

$$
\begin{aligned}
& \bar{J}_{\tilde{\Omega}_{k}}^{\prime}\left(v_{k}\right)\left(v_{k} \psi\right)=\int_{\tilde{\Omega}_{k}}\left(\left|\nabla v_{k}\right|^{2} \psi+v_{k} \nabla v_{k} \nabla \psi+v_{k}^{2} \psi\right) \mathrm{d} x-\int_{\tilde{\Omega}_{k}} f\left(v_{k}^{2}\right) v_{k}^{2} \psi \mathrm{d} x=0, \\
& \bar{J}_{\tilde{\Omega}_{k}}^{\prime}\left(v_{k}\right)(v \psi)=\int_{\tilde{\Omega}_{k}}\left(\psi \nabla v_{k} \nabla v+v \nabla v_{k} \nabla \psi+v_{k} v \psi\right) \mathrm{d} x-\int_{\tilde{\Omega}_{k}} f\left(v_{k}^{2}\right) v_{k} v \psi \mathrm{d} x=0,
\end{aligned}
$$

onde $\bar{J}_{\tilde{\Omega}_{k}}: H^{1}\left(\tilde{\Omega}_{k}, \mathbb{R}\right) \rightarrow \mathbb{R}$ é o funcional energia associado ao problema $\left(P_{5}\right)$. Combinando (4.17) e (4.18),

$$
\begin{aligned}
0 \leq & \int_{D}\left|\nabla v_{k}-\nabla v\right|^{2} \mathrm{~d} x \leq \int_{\mathbb{R}^{N}} \psi\left(\left|\nabla v_{k}\right|^{2}-2 \nabla v_{k} \nabla v+|\nabla v|^{2}\right) \mathrm{d} x \\
= & \int_{\mathbb{R}_{+}^{N}}\left[\psi\left|\nabla v_{k}\right|^{2}-\psi \nabla v_{k} \nabla v+\psi \nabla v\left(\nabla v-\nabla v_{k}\right)\right] \mathrm{d} x \\
= & \int_{\mathbb{R}_{+}^{N}}\left[f\left(v_{k}^{2}\right) v_{k}^{2} \psi-v_{k} \nabla v_{k} \nabla \psi-v_{k}^{2} \psi\right] \mathrm{d} x+\int_{\mathbb{R}_{+}^{N}}\left[v \nabla v_{k} \nabla \psi+v_{k} v \psi-f\left(v_{k}^{2}\right) v_{k} v \psi\right] \mathrm{d} x \\
& +\int_{\mathbb{R}_{+}^{N}} \psi \nabla v\left(\nabla v-\nabla v_{k}\right) \mathrm{d} x \\
= & \int_{\mathbb{R}_{+}^{N}}\left[f\left(v_{k}^{2}\right) \psi v_{k}\left(v_{k}-v\right)-\left(v_{k}-v\right) \nabla v_{k} \nabla \psi-v_{k} \psi\left(v_{k}-v\right)+\psi \nabla v\left(\nabla v_{k}-\nabla v\right)\right] \mathrm{d} x .
\end{aligned}
$$


96Capítulo 4 - Multiplicidade de soluções via teoria de Morse: condição de fronteira mista-caso geral

Isto e o fato de $\left(v_{k}\right)$ ser limitada em $L^{2}\left(\mathbb{R}_{+}^{N}, \mathbb{R}\right)$ combinado com $\left(f_{2}\right),(4.15)$ e a desigualdade de Hölder dá-nos que

$$
\int_{K}\left|\nabla v_{k}-\nabla v\right|^{2} \mathrm{~d} x \leq o_{k}(1) \text { quando } k \rightarrow \infty,
$$

isto é, $\nabla v_{k} \rightarrow \nabla v$ em $\left(L_{l o c}^{2}\left(\mathbb{R}_{+}^{N}, \mathbb{R}\right)\right)^{N}$ quando $k \rightarrow \infty$. Como consequência,

$$
\nabla v_{k}(x) \rightarrow \nabla v(x), \text { quase sempre em } \mathbb{R}_{+}^{N} \text {, quando } k \rightarrow \infty \text {. }
$$

Para finalizarmos a demonstração, resta-nos ver que

$$
\int_{\mathbb{R}_{+}^{N}}(\nabla v \nabla \phi+v \phi) \mathrm{d} x=\int_{\mathbb{R}_{+}^{N}} f\left(v^{2}\right) v \phi \mathrm{d} x, \quad \forall \phi \in C_{c}^{\infty}\left(\mathbb{R}^{N}\right) .
$$

De fato, como o conjunto das restrições das funções de $C_{c}^{\infty}\left(\mathbb{R}^{N}\right)$ a $\mathbb{R}_{+}^{N}$ é um subespaço denso de $H^{1}\left(\mathbb{R}_{+}^{N}, \mathbb{R}\right)(\operatorname{ver}[13$, Corollaire IX.8]), é suficiente mostramos que (4.20) vale para toda $\phi \in C_{c}^{\infty}\left(\mathbb{R}^{N}\right)$. Dada $\phi \in C_{c}^{\infty}\left(\mathbb{R}^{N}\right)$ qualquer, seja $t>0$ tal que $B_{t}(0) \supset \operatorname{supp} \phi$. Por (3.1), $\chi_{\tilde{\Omega}_{k} \cap B_{t}} \rightarrow \chi_{B_{t}^{+}}$quase sempre em $\mathbb{R}^{N}$, quando $k \rightarrow \infty$, onde $B_{t}^{+}:=B_{t}(0) \cap \mathbb{R}_{+}^{N}$ e $\chi_{B_{t}^{+}}$é a função característica de $B_{t}^{+}$. Isto e (4.19) implicam que $\chi_{\tilde{\Omega}_{k} \cap B_{t}}(x) \nabla v_{k}(x) \rightarrow \chi_{B_{t}^{+}}(x) \nabla v(x)$ quase sempre em $\mathbb{R}_{+}^{N}$, quando $k \rightarrow \infty$. Além disso, $\left(\chi_{\tilde{\Omega}_{k} \cap B_{t}} \nabla v_{k}\right)_{k}$ é limitada em $\left(L^{2}\left(\mathbb{R}_{+}^{N}, \mathbb{R}\right)\right)^{N} . \operatorname{Logo} \chi_{\tilde{\Omega}_{k} \cap B_{t}} \nabla v_{k} \rightarrow \chi_{B_{t}^{+}} \nabla v$ em $\left(L^{2}\left(\mathbb{R}_{+}^{N}, \mathbb{R}\right)\right)^{N}$, quando $k \rightarrow \infty$, e daí

$$
\lim _{k \rightarrow \infty} \int_{\tilde{\Omega}_{k}} \nabla v_{k} \nabla \phi \mathrm{d} x=\lim _{k \rightarrow \infty} \int_{\mathbb{R}_{+}^{N}} \chi_{\tilde{\Omega}_{k} \cap B_{t}} \nabla v_{k} \nabla \phi \mathrm{d} x=\int_{\mathbb{R}_{+}^{N}} \chi_{B_{t}^{+}} \nabla v \nabla \phi \mathrm{d} x=\int_{\mathbb{R}_{+}^{N}} \nabla v \nabla \phi \mathrm{d} x .
$$

Usando os limites (4.15) e o fato de $\phi \in C_{c}^{\infty}\left(\mathbb{R}^{N}\right)$,

$$
\int_{\tilde{\Omega}_{k}} v_{k} \phi \mathrm{d} x \rightarrow \int_{\mathbb{R}_{+}^{N}} v \phi \mathrm{d} x, \text { quando } k \rightarrow \infty .
$$

Temos $f\left(v_{k}^{2}\right) v_{k} \rightarrow f\left(v^{2}\right) v$ em $L^{\frac{q}{q-1}}\left(B_{t}^{+}, \mathbb{R}\right)$, pois, por $\left(f_{2}\right), \sup _{k}\left\|v_{k}\right\|_{D_{k}}<\infty$ e imersões de Sobolev, $\sup _{k}\left|f\left(v_{k}^{2}\right) v_{k}\right|_{\frac{q}{q-1}, B_{t}^{+}}<\infty$ e $f\left(v_{k}^{2}(x)\right) v_{k}(x) \rightarrow f\left(v^{2}(x)\right) v(x)$ quase sempre em $\mathbb{R}_{+}^{N}$. Logo, como $\operatorname{supp} \phi \subset B_{t}(0)$ e $\phi \in L^{q}\left(B_{t}^{+}, \mathbb{R}\right)$,

$$
\lim _{k \rightarrow \infty} \int_{\tilde{\Omega}_{k}} f\left(v_{k}^{2}\right) v_{k} \phi=\lim _{k \rightarrow \infty} \int_{B_{t}^{+}} f\left(v_{k}^{2}\right) v_{k} \phi=\int_{B_{t}(0)} f\left(v^{2}\right) v \phi \mathrm{d} x=\int_{\mathbb{R}_{+}^{N}} f\left(v^{2}\right) v \phi \mathrm{d} x .
$$

Por (4.21) - (4.23) e pelo fato de $v_{k}$ satisfazer $\left(P_{5}\right)$,

$$
0=\lim _{k \rightarrow \infty} \int_{\tilde{\Omega}_{k}}\left(\nabla v_{k} \nabla \phi+v_{k} \phi-f\left(v_{k}^{2}\right) v_{k} \phi\right) \mathrm{d} x=\int_{\mathbb{R}_{+}^{N}}\left(\nabla v \nabla \phi+v \phi-f\left(v^{2}\right) v \phi\right) \mathrm{d} x .
$$

Como $\phi \in C_{c}^{\infty}\left(\mathbb{R}^{N}\right)$ é qualquer, $v$ satisfaz $\left(P_{1}\right)$ no sentido fraco. 
No que segue, concluiremos a demonstração da Proposição 4.10. Tendo em vista (4.8) e (4.9),

$$
\begin{aligned}
c_{\infty}+o_{k}(1) & \geq \bar{c}_{k}=\bar{c}_{\tilde{\Omega}_{k}} \\
& =\bar{J}_{\tilde{\Omega}_{k}}\left(v_{k}\right)=\bar{J}_{\tilde{\Omega}_{k}}\left(v_{k}\right)-\frac{1}{2} \bar{J}_{\tilde{\Omega}_{k}}^{\prime}\left(v_{k}\right) v_{k} \\
& =\frac{1}{2} \int_{\tilde{\Omega}_{k}}\left[f\left(v_{k}^{2}\right) v_{k}^{2}-F\left(v_{k}^{2}\right)\right] \mathrm{d} x .
\end{aligned}
$$

Pelo Lema de Fatou e (4.15),

$$
\begin{aligned}
c_{\infty} & \geq \limsup _{k \rightarrow \infty} \bar{c}_{\tilde{\Omega}_{k}} \geq \liminf _{k \rightarrow \infty} \bar{c}_{\tilde{\Omega}_{k}} \\
& =\liminf _{k \rightarrow \infty} \frac{1}{2} \int_{\tilde{\Omega}_{k}}\left[f\left(v_{k}^{2}\right) v_{k}^{2}-F\left(v_{k}^{2}\right)\right] \mathrm{d} x \\
& \geq \frac{1}{2} \int_{\mathbb{R}_{+}^{N}}\left[f\left(v^{2}\right) v^{2}-F\left(v^{2}\right)\right] \mathrm{d} x=J_{\infty}(v) \geq c_{\infty} .
\end{aligned}
$$

Portanto, como estes argumentos podem ser aplicados a qualquer subsequência de $\left(\bar{c}_{\lambda}\right)_{\lambda}$,

$$
\lim _{\lambda \rightarrow \infty} \bar{c}_{\lambda}=c_{\infty}
$$

\subsection{A aplicação baricentro}

Para $q \in\left(2,2^{*}\right)$ dado em $\left(f_{5}\right)$ e $\lambda>0$, definimos a aplicação baricentro $\beta_{\lambda}: M_{\lambda} \rightarrow \mathbb{R}^{N}$ por

$$
\beta_{\lambda}(u):=\frac{\int_{\Omega_{\lambda}} x|u|^{q} \mathrm{~d} x}{\int_{\Omega_{\lambda}}|u|^{q} \mathrm{~d} x} .
$$

Proposição 4.13. Existem $\epsilon^{*}>0, \lambda_{1}>0$ tais que $\beta_{\lambda}(u) \in \Gamma_{1 \lambda}^{+}$, desde que $\lambda>\lambda_{1}, u \in M_{\lambda}$ e $I_{\lambda}(u) \leq b_{\lambda}^{*}$, onde $b_{\lambda}^{*}:=b_{\lambda}+\epsilon^{*}$.

\section{Demonstração.}

Mostremos que se $\left(\epsilon_{n}\right)_{n},\left(\lambda_{n}\right)_{n}$ são sequências quaisquer com $\lim _{n \rightarrow \infty} \epsilon_{n}=0, \lim _{n \rightarrow \infty} \lambda_{n}=\infty$ e $u_{n} \in M_{\lambda_{n}}$ é tal que

$$
b_{\lambda_{n}} \leq I_{\lambda_{n}}\left(u_{n}\right) \leq b_{\lambda_{n}}+\epsilon_{n}
$$

então

$$
\operatorname{dist}\left(\beta_{\lambda_{n}}\left(u_{n}\right), \Gamma_{1 \lambda_{n}}\right) \leq \lambda_{n} r,
$$

para $n$ suficientemente grande. De fato, por (4.24) e pela Proposição 4.10,

$$
b_{\lambda_{n}} \leq I_{\lambda_{n}}\left(u_{n}\right) \leq b_{\lambda_{n}}+\epsilon_{n}
$$


e daí, pela Proposição 4.10,

$$
I_{\lambda_{n}}\left(u_{n}\right) \rightarrow c_{\infty} \text { quando } n \rightarrow \infty
$$

Pela desigualdade diamagnética, pelo fato de $u_{n} \in M_{\lambda_{n}}$ e por $\left(f_{1}\right)-\left(f_{4}\right)$, existe $t_{n}>0$ tal que

$$
o_{n}(1)+c_{\infty}=I_{\lambda_{n}}\left(u_{n}\right) \geq \max _{t \geq 0} I_{\lambda_{n}}\left(t u_{n}\right) \geq \max _{t \geq 0} J_{\lambda_{n}}\left(t\left|u_{n}\right|\right)=J_{\lambda_{n}}\left(t_{n}\left|u_{n}\right|\right) \geq c_{\lambda_{n}}
$$

onde $J_{\lambda_{n}}$ é o funcional associado ao problema $\left(P_{3}\right) \operatorname{com} \lambda=\lambda_{n}$ e $c_{\lambda_{n}}$ é o nível do passo da montanha correspondente. Combinando (4.9) e (4.10) com (4.26) e a Proposição 4.10, obtemos

$$
\lim _{n \rightarrow \infty} c_{\lambda_{n}}=\lim _{n \rightarrow \infty} J_{\lambda_{n}}\left(t_{n}\left|u_{n}\right|\right)=c_{\infty}
$$

Consideremos $\bar{\epsilon}_{n}:=b_{\lambda_{n}}-c_{\lambda_{n}}$. Pela Proposição 4.10 e (4.26),

$$
\bar{\epsilon}_{n}+c_{\lambda_{n}} \geq J_{\lambda_{n}}\left(t_{n}\left|u_{n}\right|\right) \geq c_{\lambda_{n}}
$$

Aplicando o Princípio Variacional de Ekeland [25, Corollary 3.4], para todo $n \in \mathbb{N}$, existe $v_{n} \in N_{\lambda_{n}}$ tal que

$$
\left\|t_{n}\left|u_{n}\right|-v_{n}\right\|_{\Omega_{n}} \leq 2 \sqrt{\bar{\epsilon}_{n}}, \quad c_{\lambda_{n}} \leq J_{\lambda_{n}}\left(v_{n}\right) \leq c_{\lambda_{n}}+2 \bar{\epsilon}_{n}
$$

e

$$
\left\|\left(J_{\lambda_{n}} \mid N_{\lambda_{n}}\right)^{\prime}\left(v_{n}\right)\right\|_{\left(H^{1}\left(\Omega_{\lambda_{n}}, \Gamma_{0 \lambda_{n}}\right)\right)^{\prime}} \leq 8 \sqrt{\bar{\epsilon}_{n}} .
$$

Como na prova do Lema 4.6, $v_{n} \in H^{1}\left(\Omega_{\lambda_{n}}, \Gamma_{0 \lambda_{n}}\right)$ satisfaz

$$
J_{\lambda_{n}}\left(v_{n}\right) \rightarrow c_{\infty}, J_{\lambda_{n}}^{\prime}\left(v_{n}\right) \rightarrow 0 \text { quando } n \rightarrow \infty
$$

De (4.29) e $\left(f_{3}\right)$, a sequência $\left(\left\|v_{n}\right\|_{\Omega_{\lambda_{n}}}\right)_{n}$ é limitada. Consequentemente, pelo Lema 4.9 e (4.29), existem $l>0, \gamma>0$ e $y_{n} \in \mathbb{R}^{N}$ tais que

$$
\liminf _{n \rightarrow \infty} \int_{B_{l}\left(y_{n}\right) \cap \Omega_{\lambda_{n}}}\left|v_{n}\right|^{2} \geq \gamma>0 .
$$

Procedendo como na demonstração do Lema 4.11, usando (4.29) no lugar de (4.11), obtemos uma constante positiva $C>0$ tal que $\operatorname{dist}\left(y_{n}, \partial \Omega_{\lambda_{n}}\right) \leq C$. Assim, aumentando $l$ se necessário, podemos admitir que $y_{n} \in \partial \Omega_{\lambda_{n}}$. Seguindo o mesmo argumento da prova da Proposição 4.10, definamos

$$
\tilde{v}_{n}(x)=v_{n}\left(T_{y_{n}}^{-1} x+y_{n}\right), \quad \forall x \in \tilde{\Omega}_{n}:=T_{y_{n}}\left(\Omega_{\lambda_{n}}-y_{n}\right), \quad \forall n \in \mathbb{N} .
$$

para conseguirmos uma subsequênia de $\tilde{v}_{n} \in H^{1}\left(\tilde{\Omega}_{n}, \tilde{\Gamma}_{0 \lambda_{n}}\right)$, a qual denotaremos ainda por $\tilde{v}_{n}$, e uma função $v \in H^{1}\left(\mathbb{R}_{+}^{N}, \mathbb{R}\right)$ tais que

$$
\tilde{v}_{k} \rightarrow v \mathrm{em} H_{l o c}^{1}\left(\mathbb{R}_{+}^{N}, \mathbb{R}\right), \tilde{v}_{k} \rightarrow v \text { em } L_{l o c}^{p}\left(\mathbb{R}_{+}^{N}, \mathbb{R}\right), \forall p \in\left[1,2^{*}\right), \text { quando } n \rightarrow \infty,
$$




$$
\tilde{v}_{k}(x) \rightarrow v(x), \nabla \tilde{v}_{k}(x) \rightarrow \nabla v(x) \text { quase sempre em } \mathbb{R}_{+}^{N} \text {, quando } n \rightarrow \infty \text {. }
$$

Afirmação I. Existe uma constante $C>0$ tal que $\operatorname{dist}\left(y_{n}, \Gamma_{1 \lambda_{n}}\right) \leq C$.

De fato, suponhamos por contradição que a Afirmação I é falsa. Então existem subsequências, cujas notações serão mantidas,

$$
\alpha:=\operatorname{dist}\left(y_{n}, \Gamma_{1 \lambda_{n}}\right) \rightarrow \infty \text { quando } n \rightarrow \infty \text {. }
$$

A seguir, vejamos que $v \in H^{1}\left(\mathbb{R}_{+}^{N}, \mathbb{R}\right)$ satisfaz o problema

$$
\left\{\begin{aligned}
-\Delta v+v & =f\left(v^{2}\right) v, \text { em } \mathbb{R}_{+}^{N} \\
v & =0, \text { sobre } \mathbb{R}^{N-1}
\end{aligned}\right.
$$

Com efeito, definamos

$$
w_{k}(x):=\xi\left(\frac{|x|}{\alpha_{k}}\right) \tilde{v}_{k}(x), \quad \forall x \in \tilde{\Omega}_{k},
$$

onde $\alpha_{k}>0$ é dado em (4.32) e $\xi \in C_{c}^{\infty}\left(\mathbb{R}_{+}\right)$é tal que $\xi(t)=1, t \in\left[0, \frac{1}{2}\right], \xi(t)=0, t \geq \frac{2}{3}$. Assim, $w_{n} \in H_{0}^{1}\left(\tilde{\Omega}_{n}, \mathbb{R}\right) \subset H_{0}^{1}\left(\mathbb{R}_{+}^{N} v\right)$ e $w_{n}(x) \rightarrow v(x)$ quase sempre em $\mathbb{R}_{+}^{N}$ quando $n \rightarrow \infty$. Como $\left(w_{n}\right) \subset H_{0}^{1}\left(\mathbb{R}_{+}^{N}, \mathbb{R}\right)$ é limitada, existe $w \in H_{0}^{1}\left(\mathbb{R}_{+}^{N}, \mathbb{R}\right)$ tal que $w_{n} \rightarrow w$ em $H_{0}^{1}\left(\mathbb{R}_{+}^{N}, \mathbb{R}\right)$. Como consequência da imersão de Sobolev, $w_{n}(x) \rightarrow w(x)$ quase sempre em $\mathbb{R}_{+}^{N}$ quando $n \rightarrow \infty$. Da unicidade do limite quase sempre, $v \equiv w$ em $H_{0}^{1}\left(\mathbb{R}_{+}^{N}, \mathbb{R}\right)$. Tomando $\phi \in C_{c}^{\infty}\left(\mathbb{R}_{+}^{N}\right)$, temos supp $\phi \subset \tilde{\Omega}_{n}$ para $n$ suficientemente grande. Por (4.29) e da definição de $\tilde{v}_{n}$, temos

$$
\int_{\tilde{\Omega}_{n}}\left(\nabla \tilde{v}_{n} \nabla \phi+\tilde{v}_{n} \phi-f\left(\tilde{v}_{n}^{2}\right) \tilde{v}_{n} \phi\right) \mathrm{d} x=o_{n}(1) \phi
$$

para todo $n$ suficientemente grande. Por (4.30), passando ao limite $n \rightarrow \infty$ em (4.33), obtemos

$$
\int_{\mathbb{R}_{+}^{N}}\left(\nabla v \nabla \phi+v \phi-f\left(v^{2}\right) v \phi\right) \mathrm{d} x=0 .
$$

Da arbitrariedade da escolha de $\phi, v$ é solução do problema $\left(P_{6}\right)$. Seja $J_{\tilde{\Omega}_{n}}: H^{1}\left(\tilde{\Omega}_{n}, \tilde{\Gamma}_{0 \lambda_{n}}\right) \rightarrow \mathbb{R}$ o funcional energia associado ao problema

$$
\left\{\begin{aligned}
-\Delta v+v & =f\left(v^{2}\right) v, \text { em } \tilde{\Omega}_{n} \\
\frac{\partial v}{\partial \nu} & =0, \text { sobre } \tilde{\Gamma}_{1 \lambda_{n}}, \\
v & =0, \text { sobre } \tilde{\Gamma}_{0 \lambda_{n}} .
\end{aligned}\right.
$$

Do fato de $v$ ser solução de $\left(P_{6}\right)$, pelo Lema de Fatou e (4.29), temos

$$
\begin{aligned}
c_{\infty} & =\liminf _{n \rightarrow \infty} J_{\tilde{\Omega}_{n}}\left(\tilde{v}_{n}\right)=\liminf _{n \rightarrow \infty} \frac{1}{2} \int_{\tilde{\Omega}_{n}}\left(f\left(\tilde{v}_{n}^{2}\right) \tilde{v}_{n}^{2}-F\left(\tilde{v}_{n}^{2}\right)\right) \mathrm{d} x \\
& =\liminf _{n \rightarrow \infty} \frac{1}{2} \int_{\mathbb{R}_{+}^{N}}\left(\chi_{\tilde{\Omega}_{n}} f\left(\tilde{v}_{n}^{2}\right) \tilde{v}_{n}^{2}-F\left(\tilde{v}_{n}^{2}\right)\right) \mathrm{d} x \geq \frac{1}{2} \int_{\mathbb{R}_{+}^{N}}\left(f\left(v^{2}\right) v^{2}-F\left(v^{2}\right)\right) \mathrm{d} x \\
& \geq c_{\mathbb{R}_{+}^{N}} \geq c_{\infty},
\end{aligned}
$$


isto é, $c_{\infty}=c_{\mathbb{R}_{+}^{N}}$. Mas $c_{\infty}=c_{\mathbb{R}_{+}^{N}} \geq c_{\mathbb{R}^{N}}=2 c_{\infty}$, o que é um absurdo. Dessa forma, a afirmação está mostrada.

Afirmação II. Dado $\epsilon>0$ qualquer, existe $R=R(\epsilon)>0$ tal que

$$
\lim _{n \rightarrow \infty} \frac{1}{2} \int_{\Omega_{n} \cap B_{R}\left(y_{n}\right)}\left(f\left(v_{n}^{2}\right) v_{n}^{2}-F\left(v_{n}^{2}\right)\right) \mathrm{d} x \geq c_{\infty}-\epsilon .
$$

De fato, primeiramente mostraremos que a função $v$ dada em (4.30)-(4.31) satisfaz $J_{\infty}(v)=c_{\infty}$ é solução de $\left(P_{1}\right)$. Seja $\phi \in C_{c}^{\infty}\left(\mathbb{R}^{N}\right)$ tal que $\phi=1 \mathrm{em} B_{1}(0), \phi=0$ em $B_{2}^{c}(0)$, $0 \leq \phi \leq 1$, e definamos

$$
\phi_{T}(x):=\phi\left(\frac{x}{T}\right), \quad \forall x \in \mathbb{R}^{N}, T>0 .
$$

Então a sequencia $\phi_{T} \tilde{v}_{n}$ é limitada em $H^{1}\left(\tilde{\Omega}_{n}, \tilde{\Gamma}_{0 \lambda_{n}}\right.$ e $\phi_{T} v \rightarrow v$ em $H^{1}\left(\mathbb{R}_{+}^{N}, \mathbb{R}\right)$ quando $T \rightarrow \infty$. Por (4.29), temos

$$
\int_{\tilde{\Omega}_{n}}\left(\nabla \tilde{v}_{n} \nabla\left(\phi_{T} \tilde{v}_{n}\right)+\left|\tilde{v}_{n}\right|^{2} \phi_{T}\right) \mathrm{d} x=\int_{\tilde{\Omega}_{n}}\left(f\left(\tilde{v}_{n}^{2}\right) \tilde{v}_{n}^{2} \phi_{T}\right) \mathrm{d} x+o_{n}(1)
$$

isto é,

$$
\int_{\tilde{\Omega}_{n}}\left|\nabla \tilde{v}_{n}\right|^{2} \phi_{T} \mathrm{~d} x+\int_{\tilde{\Omega}_{n}} \tilde{v}_{n} \nabla \tilde{v}_{n} \nabla \phi_{T} \mathrm{~d} x+\int_{\tilde{\Omega}_{n}}\left|\tilde{v}_{n}\right|^{2} \phi_{T} \mathrm{~d} x=\int_{\tilde{\Omega}_{n}} f\left(\tilde{v}_{n}^{2}\right) \tilde{v}_{n}^{2} \phi_{T} \mathrm{~d} x+o_{n}(1) .
$$

Mostremos que

$$
\begin{aligned}
& \int_{\tilde{\Omega}_{n}} \tilde{v}_{n} \nabla \tilde{v}_{n} \nabla \phi_{T} \mathrm{~d} x \rightarrow \int_{\mathbb{R}_{+}^{N}} v \nabla v \nabla \phi_{T} \mathrm{~d} x, \\
& \int_{\tilde{\Omega}_{n}}\left|\tilde{v}_{n}\right|^{2} \phi_{T} \mathrm{~d} x \rightarrow \int_{\mathbb{R}_{+}^{N}}|v|^{2} \phi_{T} \mathrm{~d} x \\
& \int_{\tilde{\Omega}_{n}} f\left(\tilde{v}_{n}^{2}\right) \tilde{v}_{n}^{2} \phi_{T} \mathrm{~d} x \rightarrow \int_{\mathbb{R}_{+}^{N}} f\left(v^{2}\right) v^{2} \phi_{T} \mathrm{~d} x,
\end{aligned}
$$

quando $n \rightarrow \infty$. Sejam $\epsilon>0$ e $T>1$ quaisquer. Fixemos $t>0$ a ser escolhido posteriormente e definamos

$$
E_{t}:=\left\{x \in B_{2 T}(0) ; 0 \leq x^{N} \leq t\right\}
$$

Usando que $\left(\left\|\tilde{v}_{n}\right\|_{\tilde{\Omega}_{n}}\right)_{n}$ é limitada e a desigualdade de Holder, obtemos

$$
\int_{E_{t}}\left|\tilde{v}_{n}\right|^{2} \mathrm{~d} x=\left(\int_{E_{t}}\left|\tilde{v}_{n}\right|^{2^{*}} \mathrm{~d} x\right)^{\frac{2}{2^{*}}}\left(\int_{E_{t}} 1^{N} \mathrm{~d} x\right)^{\frac{2}{2^{*}}} \leq M\left|E_{t}\right|^{\frac{2}{N}} \leq M T^{(N-1) \frac{2}{N}} t^{\frac{2}{N}}
$$

e

$$
\int_{E_{t}}\left|\tilde{v}_{n}\right|^{q} \mathrm{~d} x=\left(\int_{E_{t}}\left|\tilde{v}_{n}\right|^{2^{*}} \mathrm{~d} x\right)^{\frac{q}{2^{*}}}\left(\int_{E_{t}} 1^{\alpha} \mathrm{d} x\right)^{\frac{q}{\alpha}} \leq M\left|E_{t}\right|^{\frac{q}{\alpha}} \leq M T^{(N-1) \frac{q}{\alpha}} t^{\frac{q}{\alpha}},
$$

para alguma constante $M>0$, onde $\alpha=\frac{2^{*}}{2^{*}-q}$. Ponhamos $\kappa:=\max \{\alpha / q, N / 2\}$ e tomemos $t=\epsilon^{\kappa} T^{N-1}$. Logo

$$
T^{(N-1) \frac{2}{N}} t^{\frac{2}{N}}=\epsilon^{\kappa \frac{2}{N}} \quad \text { e } \quad T^{(N-1) \frac{q}{\alpha}} t^{\frac{q}{\alpha}}=\epsilon^{\kappa \frac{q}{\alpha}}, \quad \operatorname{com} \min \{\kappa 2 / N, \kappa q / \alpha\}=1>0 .
$$


donde

$$
\lim _{\epsilon \rightarrow 0} \epsilon^{\kappa \frac{q}{\alpha}}=\lim _{\epsilon \rightarrow 0} \epsilon^{\kappa \frac{2}{N}}=0 .
$$

Assim, pela escolha de $t$, obtemos

$$
\int_{E_{t}}\left|\tilde{v}_{k}\right|^{2} \leq \epsilon^{\kappa \frac{2}{N}} M \quad \text { e } \quad \int_{E_{t}}\left|\tilde{v}_{k}\right|^{q} \leq \epsilon^{\kappa \frac{q}{\alpha}} M .
$$

Vale ressaltar que, pelo Lema de Fatou e (4.31), $v$ também satisfaz (4.39). Além disso, $B_{2 T} \backslash E_{t} \subset \tilde{\Omega}_{n}$, para $n$ suficientemente grande. Aplicando a desigualdade de Holder, (4.30), (4.31) e (4.39), para todo $n$ suficientemente grande, temos

$$
\begin{aligned}
& \left|\int_{\tilde{\Omega}_{n}} \tilde{v}_{n} \nabla \phi_{T} \nabla \tilde{v}_{n} \mathrm{~d} x-\int_{\mathbb{R}_{+}^{N}} v \nabla \phi_{T} \nabla v\right| \mathrm{d} x \leq \\
& \quad \leq\left|\int_{B_{2 T} \backslash E_{t}}\left(\tilde{v}_{n} \nabla \phi_{T} \nabla \tilde{v}_{n}-v \nabla \phi_{T} \nabla v\right) \mathrm{d} x\right|+\int_{E_{t}}\left(\left|\tilde{v}_{n} \nabla \phi_{T} \nabla \tilde{v}_{n}\right|+\left|v \nabla \phi_{T} \nabla v\right|\right) \mathrm{d} x \\
& \quad \leq\left|\int_{B_{2 T} \backslash E_{t}} \nabla \phi_{T} \nabla \tilde{v}_{n}\left(\tilde{v}_{n}-v\right) \mathrm{d} x\right|+\left|\int_{B_{2 T} \backslash E_{t}} v \nabla \phi_{T}\left(\nabla \tilde{v}_{n}-\nabla v\right) \mathrm{d} x\right|+M \int_{E_{t}}\left(\left|\tilde{v}_{n}\right|^{2}+|v|^{2}\right) \mathrm{d} x \\
& \quad \leq o_{n}(1)+M\left(\epsilon^{\kappa \frac{q}{\alpha}}+\epsilon^{\kappa \frac{2}{N}}\right) .
\end{aligned}
$$

Por (4.38) e pelo fato de que podemos escolher $\epsilon>0$ arbitrariamente pequeno, (4.35) é válido para todo $T>1$. Argumentando de forma análoga, concluímos (4.36). Sobre (4.37), combinamos $\left(f_{6}\right)$ e (4.39) para obtermos

$$
\int_{E_{t}} f\left(\tilde{v}_{k}^{2}\right) \tilde{v}_{k}^{2} \mathrm{~d} x \leq \epsilon \int_{E_{t}} \tilde{v}_{k}^{2} \mathrm{~d} x+c_{\epsilon} \int_{E_{t}} \tilde{v}_{k}^{q} \mathrm{~d} x \leq M\left(\epsilon^{\kappa \frac{q}{\alpha}}+\epsilon^{\kappa \frac{2}{N}}\right)
$$

Vale ressaltar que, sem perda de generalidade, $v$ satisfaz a estimativa acima. Por (4.30) e (4.40), temos

$$
\begin{aligned}
\left|\int_{\tilde{\Omega}_{n}} f\left(\tilde{v}_{n}^{2}\right) \tilde{v}_{n}^{2} \phi_{T} \mathrm{~d} x-\int_{\mathbb{R}_{+}^{N}} f\left(v^{2}\right) v^{2} \phi_{T} \mathrm{~d} x\right| \leq \\
\quad \leq\left|\int_{B_{2 T} \backslash E_{t}}\left(f\left(\tilde{v}_{n}^{2}\right) \tilde{v}_{n}^{2}-f\left(v^{2}\right) v^{2}\right) \phi_{T} \mathrm{~d} x\right|+\mid \int_{E_{t}}\left(f\left(\tilde{v}_{n}^{2}\right) \tilde{v}_{n}^{2}|+| f\left(v^{2}\right) v^{2} \mid\right) \mathrm{d} x \\
\quad \leq o_{n}(1)+M\left(\epsilon^{\kappa \frac{q}{\alpha}}+\epsilon^{\kappa \frac{2}{N}}\right) .
\end{aligned}
$$

Portanto, como na conclusão de (4.35), segue (4.37) para todo $T>1$. Combinando (4.34)-(4.37) e o Lema de Fatou, obtemos

$$
\int_{\mathbb{R}_{+}^{N}}|\nabla v|^{2} \phi_{T}+\int_{\mathbb{R}_{+}^{N}} v \nabla v \nabla \phi_{T}+\int_{\mathbb{R}_{+}^{N}}|v|^{2} \phi_{T} \leq \int_{\mathbb{R}_{+}^{N}} f\left(v^{2}\right) v^{2} \phi_{T},
$$

para todo $T>1$. Finalmente, tomando o limite $T \rightarrow \infty$, encontramos

$$
\int_{\mathbb{R}_{+}^{N}}\left(|\nabla v|^{2}+v^{2}\right) \leq \int_{\mathbb{R}_{+}^{N}} f\left(v^{2}\right) v^{2} \mathrm{~d} x
$$


Por $\left(f_{1}\right)-\left(f_{4}\right)$, existe $t_{0}>0$ tal que $t_{0} v \in N_{\infty}$. Por (4.41), $0<t_{0} \leq 1$. Suponha por contradição que $t_{0}<1$. Neste caso, usando que a função $s \mapsto f(s) s-F(s)$ é crescente em $[0,+\infty)$, por $\left(f_{4}\right)$, Lema de Fatou e (4.31),

$$
\begin{aligned}
c_{\infty} & =\liminf _{n \rightarrow \infty} J_{\tilde{\Omega}_{n}}\left(\tilde{v}_{n}\right)-\frac{1}{2} J_{\tilde{\Omega}_{n}}^{\prime}\left(\tilde{v}_{n}\right) \tilde{v}_{n}=\liminf _{n \rightarrow \infty} \frac{1}{2} \int_{\tilde{\Omega}_{n}} f\left(\tilde{v}_{n}^{2}\right) \tilde{v}_{n}^{2}-F\left(\tilde{v}_{n}^{2}\right) \\
& \geq \frac{1}{2} \int_{\mathbb{R}_{+}^{N}}\left(f\left(v^{2}\right) v^{2}-F\left(v^{2}\right)\right) \mathrm{d} x>\frac{1}{2} \int_{\mathbb{R}_{+}^{N}}\left(f\left(t_{0}^{2} v^{2}\right) t_{0}^{2} v^{2}-F\left(t_{0}^{2} v^{2}\right)\right) \mathrm{d} x \\
& =J_{\infty}\left(t_{0} v\right)-\frac{1}{2} J_{\infty}^{\prime}\left(t_{0} v\right) t_{0} v \geq c_{\infty},
\end{aligned}
$$

o que é um absurdo. Logo $t_{0}=1$ e $v \in N_{\infty}$. Mais ainda, $v$ satisfaz

$$
c_{\infty} \geq \frac{1}{2} \int_{\mathbb{R}_{+}^{N}} f\left(v^{2}\right) v^{2}-F\left(v^{2}\right)=J_{\infty}(v)-\frac{1}{2} J_{\infty}^{\prime}(v) v=J_{\infty}(v) \geq c_{\infty}
$$

ou seja, $J_{\infty}(v)=c_{\infty}$ e $v$ é uma solução de $\left(P_{1}\right)$. De (4.42), dado $\epsilon>0$ qualquer, existe $R>0$ tal que

$$
\frac{1}{2} \int_{\mathbb{R}_{+}^{N} \cap B_{R}(0)} f\left(v^{2}\right) v^{2}-F\left(v^{2}\right) \geq c_{\infty}-\epsilon .
$$

Como $\chi_{B_{R} \cap \tilde{\Omega}_{n}}(x) \tilde{v}_{n}(x) \rightarrow \chi_{B_{R}^{+}}(x) v(x)$ quase sempre em $\mathbb{R}_{+}^{N}$ quando $n \rightarrow \infty$, pelo Lema de Fatou, temos

$$
\begin{aligned}
\liminf _{n \rightarrow \infty} \frac{1}{2} \int_{B_{R}\left(y_{n}\right) \cap \Omega_{n}}\left(f\left(v_{n}^{2}\right) v_{n}^{2}-F\left(v_{n}^{2}\right)\right) \mathrm{d} x & =\liminf _{n \rightarrow \infty} \frac{1}{2} \int_{B_{R} \cap \tilde{\Omega}_{n}}\left(f\left(\tilde{v}_{n}^{2}\right) \tilde{v}_{n}^{2}-F\left(\tilde{v}_{n}^{2}\right)\right) \mathrm{d} x \\
& \geq \frac{1}{2} \int_{\mathbb{R}_{+}^{N} \cap B_{R}(0)}\left(f\left(v^{2}\right) v^{2}-F\left(v^{2}\right)\right) \mathrm{d} x \\
& \geq c_{\infty}-\epsilon
\end{aligned}
$$

e a Afirmação II está mostrada.

Agora estamos aptos a mostrar (4.25). Por (4.28) e pelas imersões de Sobolev, a sequência $\left\{t_{k}\left|u_{k}\right| \in H^{1}\left(\Omega_{k}, \Gamma_{0 \lambda_{k}}\right)\right\}_{k}$ tem o mesmo limite quase sempre de $\left\{v_{k} \in H^{1}\left(\Omega_{k}, \Gamma_{0 \lambda_{k}}\right)\right\}_{k}$.

Portanto a Afirmação II também é válida para $\left\{t_{n}\left|u_{n}\right|\right\}$, isto é,

$$
\liminf _{n \rightarrow \infty} \frac{1}{2} \int_{B_{R}\left(y_{n}\right) \cap \Omega_{\lambda_{n}}}\left(f\left(\left|t_{n} u_{n}\right|^{2}\right)\left|t_{n} u_{n}\right|^{2}-F\left(\left|t_{n} u_{n}\right|^{2}\right)\right) \mathrm{d} x \geq c_{\infty}-\epsilon,
$$

Disto, de $(4.27)$ e $\left(f_{5}\right)$, segue que

$$
\liminf _{k \rightarrow \infty} \int_{B_{R}^{c}\left(y_{k}\right) \cap \Omega_{\lambda_{k}}} c\left|t_{k} u_{k}\right|^{q} \leq \epsilon
$$

Pela Afirmação I, podemos supor que $y_{n} \in \Gamma_{1 \lambda_{n}}$, isto é, $y_{n} / \lambda_{n} \in \Gamma_{1}$ e $y_{n} / \lambda_{n} \rightarrow x_{0} \in \Gamma_{1}$ quando $n \rightarrow \infty$, uma vez que $\bar{\Gamma}_{1}$ é um conjunto compacto. Seja $j \in\{1, \ldots, N\}$. Pela 
definição do baricentro, temos

$$
\begin{aligned}
\left|\frac{\beta_{\lambda_{n}}^{j}\left(u_{n}\right)}{\lambda_{n}}-x_{0}^{j}\right| & =\left|\frac{\int_{\Omega_{\lambda_{n}}} \frac{x^{j}}{\lambda_{n}}\left|u_{n}\right|^{q} \mathrm{~d} x}{\int_{\Omega_{\lambda_{n}}}\left|u_{n}\right|^{q} \mathrm{~d} x}-\frac{\int_{\Omega_{\lambda_{n}}} x_{0}^{j}\left|u_{n}\right|^{q} \mathrm{~d} x}{\int_{\Omega_{\lambda_{n}}}\left|u_{n}\right|^{q} \mathrm{~d} x}\right| \\
& \leq \frac{\int_{\Omega_{\lambda_{n}}}\left|\frac{x^{j}}{\lambda_{n}}-x_{0}^{j}\right|\left|t_{n} u_{n}\right|^{q} \mathrm{~d} x}{\int_{\Omega_{\lambda_{n}}}\left|t_{n} u_{n}\right|^{q} \mathrm{~d} x} .
\end{aligned}
$$

Usando o Lema 3.10 e o fato de $t_{n}\left|u_{n}\right| \in M_{\lambda_{k}}$, podemos admitir que

$$
\int_{\Omega_{\lambda_{n}}}\left|t_{n} u_{n}\right|^{q} \geq \gamma>0, \quad \forall n \in \mathbb{N} .
$$

Consequentemente, por (4.44),

$$
\begin{aligned}
& \gamma\left|\frac{\beta_{\lambda_{n}}^{j}\left(u_{n}\right)}{\lambda_{n}}-x_{0}^{j}\right| \leq\left|\frac{\beta_{\lambda_{n}}^{j}\left(u_{n}\right)}{\lambda_{n}}-x_{0}^{j}\right| \int_{\Omega_{\lambda_{n}}}\left|t_{n} u_{n}\right|^{q} \mathrm{~d} x \leq \int_{\Omega_{\lambda_{n}}}\left|\frac{x^{j}}{\lambda_{n}}-x_{0}^{j}\right|\left|t_{n} u_{n}\right|^{q} \mathrm{~d} x \\
& =\int_{\Omega_{\lambda_{n}} \cap B_{R}\left(y_{n}\right)}\left|\frac{x^{j}}{\lambda_{n}}-x_{0}^{j}\right|\left|t_{n} u_{n}\right|^{q} \mathrm{~d} x+\int_{\Omega_{\lambda_{n}} \backslash B_{R}\left(y_{n}\right)}\left|\frac{x^{j}}{\lambda_{n}}-x_{0}^{j}\right|\left|t_{n} u_{n}\right|^{q} \mathrm{~d} x \\
& \leq \int_{\Omega_{\lambda_{n}} \cap B_{R}\left(y_{n}\right)}\left|\frac{x^{j}}{\lambda_{n}}-\frac{y_{n}^{j}}{\lambda_{n}}\right|\left|t_{n} u_{n}\right|^{q} \mathrm{~d} x+\int_{\Omega_{\lambda_{n}} \cap B_{R}\left(y_{n}\right)}\left|\frac{y_{n}^{j}}{\lambda_{n}}-x_{0}^{j}\right|\left|t_{n} u_{n}\right|^{q} \mathrm{~d} x+ \\
& +\int_{\Omega_{\lambda_{n}} \backslash B_{R}\left(y_{n}\right)}\left|\frac{x^{j}}{\lambda_{n}}-x_{0}^{j}\right|\left|t_{n} u_{n}\right|^{q} \mathrm{~d} x \\
& \leq \frac{R}{\lambda_{n}} \int_{\Omega_{\lambda_{n}}}\left|t_{n} u_{n}\right|^{q} \mathrm{~d} x+\left|\frac{y_{n}}{\lambda_{n}}-x_{0}\right| \int_{\Omega_{\lambda_{n}}}\left|t_{n} u_{n}\right|^{q} \mathrm{~d} x+
\end{aligned}
$$

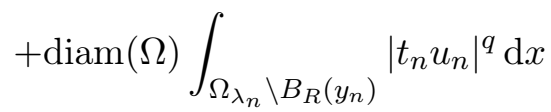

$$
\begin{aligned}
& =\left(\frac{R}{\lambda_{n}}+\left|\frac{y_{n}}{\lambda_{n}}-x_{0}\right|\right) \int_{\Omega_{n}}\left|t_{n} u_{n}\right|^{q} \mathrm{~d} x+\operatorname{diam}(\Omega) \int_{\Omega_{\lambda_{n} \backslash B_{R}\left(y_{n}\right)}}\left|t_{n} u_{n}\right|^{q} \mathrm{~d} x .
\end{aligned}
$$

De (4.43), do fato de a sequência $\left(\left\|t_{n} u_{n}\right\|\right)_{A_{\lambda_{n}}}$ ser limitada e $y_{n} / \lambda_{n} \rightarrow x_{0}$ quando $n \rightarrow \infty$, encontramos

$$
0 \leq \liminf _{n \rightarrow \infty}\left|\frac{\beta_{\lambda_{n}}^{j}\left(u_{n}\right)}{\lambda_{n}}-x_{0}^{j}\right| \leq \operatorname{diam}(\Omega) \frac{\epsilon}{\gamma C}, \quad \forall j \in\{1, \ldots, N\} .
$$

Como $\epsilon>0$ é arbitrário, podemos encontra uma subsequência, para a qual manteremos as notações, tal que

$$
\operatorname{dist}\left(\frac{\beta_{\lambda_{n}}\left(u_{n}\right)}{\lambda_{n}}, \Gamma_{1}\right) \rightarrow 0, \text { quando } n \rightarrow \infty
$$

Concluímos que $\operatorname{dist}\left(\beta_{\lambda_{n}}\left(u_{n}\right), \Gamma_{1 \lambda_{n}}\right) \leq \lambda_{n} r$, para todo $n$ suficientemente grande, donde vale (4.25) e o resultado segue. 
Seja $\epsilon^{*}$ dado na Proposição 4.13. Definamos

$$
b_{\lambda}^{*}=b_{\lambda}+\epsilon^{*} .
$$

Como consequência do Lema 4.8 e das Proposições 4.10 e 4.13, obtemos o seguinte resultado, o qual é fundamental na comparação das topologias dos subníveis do funcional $I_{\lambda}$ e de $\Gamma_{1 \lambda}$.

Lema 4.14. Existe $\lambda^{*}>0$ tal que

$$
\Phi_{\lambda}\left(\Gamma_{1 \lambda}^{-}\right) \subset M_{\lambda}^{b_{\lambda}^{*}}, \quad \beta_{\lambda}\left(M_{\lambda}^{b_{\lambda}^{*}}\right) \subset \Gamma_{1 \lambda}^{+},
$$

para $\lambda>\lambda^{*}$, onde $M_{\lambda}^{b_{\lambda}^{*}}:=I_{\lambda}^{b_{\lambda}^{*}} \cap M_{\lambda}$.

\section{Demonstração.}

Pela Proposição 4.13, existe $\lambda_{1}>0$ tal que $\beta_{\lambda}\left(M_{\lambda}^{b_{\lambda}^{*}}\right) \subset \Gamma_{1 \lambda}^{+}$. Do Lema 4.8 e Proposição 4.10 , temos

$$
\lim _{\lambda \rightarrow \infty} \max _{y \in \Gamma_{1 \lambda}^{-}}\left|I_{\lambda}\left(\Phi_{\lambda}(y)\right)-b_{\lambda}\right|=0
$$

Pela definição de limite, (4.46) implica que, para $\epsilon^{*}>0$, existe $\lambda_{2}>0$ tal que

$$
I_{\lambda}\left(\Phi_{\lambda}(y)\right) \leq b_{\lambda}+\epsilon^{*}
$$

para todo $\lambda>\lambda_{2}$ e $y \in \Gamma_{1 \lambda}^{-}$. Ponhamos $\lambda^{*}:=\max \left\{\lambda_{1}, \lambda_{2}\right\}$. Então

$$
\Phi_{\lambda}\left(\Gamma_{1 \lambda}^{-}\right) \subset M_{\lambda}^{b_{\lambda}^{*}} \quad \text { e } \quad \beta_{\lambda}\left(M_{\lambda}^{b_{\lambda}^{*}}\right) \subset \Gamma_{1 \lambda}^{+}, \quad \text { para todo } \lambda>\lambda^{*} .
$$

Vale ressaltar que, pelo lema acima, $\beta \circ \Phi_{\lambda}$ é homotopicamente equivalente à identidade em $\Gamma_{1}$.

\subsection{Prova do Teorema 4.1}

Através das aplicações $\beta_{\lambda}$ e $\Phi_{\lambda}$ definidas anteriormente, é possível compararmos as topologias de $M_{\lambda}^{b_{\lambda}^{*}}$ e $\Gamma_{1 \lambda}$. A demonstração do resultado seguinte é idêntica à do Lema 3.17 do Capítulo 3, por isso ocultá-la-emos.

Lema 4.15. Seja $\lambda^{*}>0$ dado no Lema 4.14. Então, para qualquer $\lambda>\lambda^{*}$,

$$
\operatorname{cat}\left(M_{\lambda}^{b_{\lambda}^{*}}\right) \geq \operatorname{cat}\left(\Gamma_{1 \lambda}\right)
$$

A seguir, temos a demonstração do Teorema 4.1. Ela é idêntica à apresentada no Capítulo 2. 


\section{Demonstração do Teorema 4.1.}

Tomemos $\epsilon^{*}>0$ dado na Proposição $4.13, \lambda^{*}>0$ dado na Proposição 4.14 e $\lambda>\lambda^{*}$. Se $b_{\lambda}^{*}=b_{\lambda}+\epsilon^{*}$ é um valor crítico de $I_{\lambda}$ para todo $\epsilon \in\left(0, \epsilon^{*}\right]$, então $I_{\lambda}$ possui infinitos pontos críticos e a prova está completa. Por outro lado, admitamos que $b_{\lambda}^{*}$ é um valor regular de $I_{\lambda}$. Como $M_{\lambda}^{b_{\lambda}^{*}}$ é um conjunto fechado de $M_{\lambda}$, tendo em vista as Proposição $4.6, I_{\lambda}$ restrito a $M_{\lambda}^{b_{\lambda}^{*}}$ satisfaz $(P S)_{d}$, para todo $d \in \mathbb{R}$. Logo podemos aplicar a teoria de

Ljusternik-Schnirelmann e a Proposição 4.15 para obtemos $\operatorname{cat}\left(\Gamma_{1 \lambda}\right)$ pontos críticos de $\left.I_{\lambda}\right|_{M_{\lambda}^{b_{\lambda}^{*}}}$. Pelo fato de $b_{\lambda}^{*}$ ser valor regular, obtemos $\operatorname{cat}\left(\Gamma_{1 \lambda}\right)$ pontos críticos de $\left.I_{\lambda}\right|_{M_{\lambda}}$. Pelo Corolário 4.7 , estes são pontos críticos de $I_{\lambda}$.

A próxima seção traz os resultados de Teoria de Morse necessários à demonstração dos Teorema 4.2 e Corolário 4.3, assim como a demonstração destes.

\subsection{Provas dos Teorema 4.2 e Corolário 4.3}

Nesta seção usaremos os resultados mostrados na Seção A.3 do Apêndice A. Verifiquemos, então, que as condições exigidas na referida seção são satisfeitas. De fato:

- a condição $(i)$ segue da forma como o funcional $I_{\lambda}$ foi definido e das hipóteses sobre $f$;

- (ii) segue da Proposição 4.4 e de $H_{I_{\lambda}}(u): H_{A_{\lambda}}^{1}\left(\Omega_{\lambda}, \Gamma_{0 \lambda}\right) \times H_{A_{\lambda}}^{1}\left(\Omega_{\lambda}, \Gamma_{0 \lambda}\right) \rightarrow \mathbb{R}$ dada por

$$
H_{I_{\lambda}}(u)(v, w)=\langle v, w\rangle_{A_{\lambda}}-\int_{\Omega_{\lambda}}\left(2 f^{\prime}\left(|u|^{2}\right) \operatorname{Re}(u \bar{v}) \operatorname{Re}(u \bar{w})+f\left(|u|^{2}\right) \operatorname{Re}(w \bar{v})\right) \mathrm{d} x,
$$

para $v, w \in H_{A_{\lambda}}^{1}\left(\Omega_{\lambda}, \Gamma_{0 \lambda}\right)$, ser uma forma bilinear simétrica, para todo $u \in H_{A_{\lambda}}^{1}\left(\Omega_{\lambda}, \Gamma_{0 \lambda}\right)$;

- $\left(\right.$ iii) e $\left(\right.$ iv) são consequências da Proposição 4.5 - vale ressaltar que $\delta_{0}>0$ independente de $\lambda>0$;

- $(v)$ segue pelo Lema 4.14 para $\lambda>\lambda^{*}$ : temos $\Phi_{\lambda}: \Gamma_{1 \lambda}^{-} \rightarrow M_{\lambda}^{b_{\lambda}^{*}}$ e $\beta_{\lambda}: M_{\lambda}^{b_{\lambda}^{*}} \rightarrow \Gamma_{1 \lambda}^{+}$, onde $b_{\lambda}^{*}$, valor regular de $I_{\lambda}$, é dado em (4.45), e, por construção $\beta_{\lambda} \circ \Phi_{\lambda}=I d_{\Gamma_{1 \lambda}^{-}}, \Gamma_{1 \lambda}^{+}, \Gamma_{1 \lambda}^{-} \mathrm{e}$ $\Gamma_{1 \lambda}$ homotopicamente equivalentes.

Assim, tomando, para $\lambda>\lambda^{*}$, os entes matemáticos deste capítulo que correspondem aos que foram fixados na Seção A.3 do Apêndice A, vamos às demonstrações dos resultados principais.

\section{Demonstração do Teorema 4.2.}

Seja $\lambda \geq \lambda^{*}, \lambda^{*}>0$ dado no Lema 4.14. Seja também $\mathcal{K}$ o conjunto dos pontos críticos de $I_{\lambda}$. Suponha que $\mathcal{K}$ é discreto. 
Definamos

$$
\mathcal{C}_{1}:=\left\{u \in \mathcal{K} ; \delta<I_{\lambda}(u) \leq b_{\lambda}^{*}\right\}
$$

e

$$
\mathcal{C}_{2}:=\left\{u \in \mathcal{K} ; b_{\lambda}^{*}<I_{\lambda}(u)\right\}
$$

$\operatorname{com} \delta \in\left(0, \delta_{0}\right)$ dado na Proposição $4.5, b_{\lambda}^{*}$ como em (4.45). Então, como $\mathcal{C}_{1}, \mathcal{C}_{2}$ são conjuntos críticos isolados e $\mathcal{K}=\mathcal{C}_{1} \cup \mathcal{C}_{2}$, pelas propriedades do índice de Morse generalizado

$$
i_{t}\left(\mathcal{K}, I_{\lambda}\right)=i_{t}\left(\mathcal{C}_{1},\left.I_{\lambda}\right|_{\overline{\Sigma\left(\delta, b_{\lambda}^{*}\right)}}\right)+i_{t}\left(\mathcal{C}_{2},\left.I_{\lambda}\right|_{\overline{\Sigma\left(b_{\lambda}^{*},+\infty\right)}}\right)=\sum_{u \in \mathcal{C}_{1}} i_{t}(u)+\sum_{u \in \mathcal{C}_{2}} i_{t}(u)
$$

Pelo Lema A.26,

$$
\sum_{u \in \mathcal{C}_{1}} i_{t}(u)=t \mathcal{P}_{t}\left(\Gamma_{1 \lambda}\right)+t \mathcal{Q}(t)+(1+t) \mathcal{Q}_{1}(t)
$$

e

$$
\sum_{u \in \mathcal{C}_{2}} i_{t}(u)=t^{2}\left[\mathcal{P}_{t}\left(\Gamma_{1 \lambda}\right)+\mathcal{Q}(t)-1\right]+(1+t) \mathcal{Q}_{2}(t)
$$

Então, como $\mathcal{Q}$ é um polinômio com coeficientes não negativos,

$$
\sum_{u \in \mathcal{K}} i_{t}(u)=t \mathcal{P}_{t}\left(\Gamma_{1 \lambda}\right)+t^{2}\left[\mathcal{P}_{t}\left(\Gamma_{1 \lambda}\right)-1\right]+(1+t) \mathcal{Q}_{3}(t)
$$

com $\mathcal{Q}_{3}$ é um polinômio com coeficientes não negativos..

\section{Demonstração do Corolário 4.3.}

Seja $\lambda \geq \lambda^{*}, \lambda^{*}>0$ dado na Proposição 4.14. Seja também $\mathcal{K}$ o conjunto dos pontos críticos de $I_{\lambda}$. Suponha que todos os pontos críticos de $I_{\lambda}$ são não degenerados. Basta, então, observar que, nestas circunstâncias,

$$
i(u)=t^{m(u)} \text {, para todo } u \in \mathcal{K} .
$$




\section{Teoria de Morse e resultados de} homologia

Neste apêndice daremos algumas noções gerais sobre a Teoria de Morse e resultados de Homologia que são necessários para o entendimento dos argumentos utilizados.

\section{A.1 Alguns resultados básicos de homologia}

Definição A.1. Sejam $X$ espaço topológico e $A \subseteq X$ um subespaço. Chamaremos de par topológico o par $(X, A)$.

Dado um par topológico $(X, A)$, denotaremos por $H_{q}(X, A)$ o $q$-ésimo grupo de homologia singular relativa com coeficientes em algum corpo $\mathbb{K}$.

A seguir enunciaremos alguns resultados que serão utilizados no decorrer deste apêndice. Antes disso, vejamos algumas definições auxiliares.

Definição A.2. Sejam $(X, A)$ um par topológico. Duas aplicações $f, g: X \rightarrow X$ são chamadas homotópicas se existe uma aplicação contínua $h:[0,1] \times X \rightarrow X$ tal que $h(0,)=$.$f e h(1,)=$.$g . Dizemos que h$ é uma homotopia entre $f$ e $g$.

Definição A.3. Sejam $X, Y$ espaços topológicos. Uma aplicação $f: X \rightarrow Y$ é uma equivalência homotópica se existir uma aplicação $g: Y \rightarrow X$ tal que as compostas $f \circ g$ e $g \circ f$ são homotópicas às identidades de $X$ e $Y$, respectivamente. Diremos, então, que $X$ e $Y$ 
têm o mesmo tipo de homotopia. Dizemos que um espaço é contrátil se tiver o mesmo tipo de homotopia de um ponto.

Definição A.4. Uma aplicação contínua $r: X \rightarrow A$ e uma retração se $r(x)=x, \forall x \in A$. Nestas condições, dizemos que $A$ é um retrato de $\mathrm{X}$. Se, além disso, existe uma homotopia $h:[0,1] \times X \rightarrow X$ tal que, para todo $x \in X, h(0,)=$.$x e h(1,)=.r(x)$, então dizemos que $A$ é um retrato de deformação de $X$. Dizemos ainda que $A$ é um retrato de deformação forte de $X$ se $h(t,)=$.$x para todo x \in A$.

Dados pares topológicos $(X, A),(A, B)$, tem-se:

(A.1) dim $H_{0}(X)$ é o número de componentes conexas por caminhos de $X$;

(A.2) $\operatorname{dim} H_{0}(X, A)$ é o número de componentes conexas por caminhos de $X$ que não intersectam $A$;

(A.3) se $A$ é um retrato de deformação forte de $X$, então a injeção canônica induz um isomorfismo $H_{k}(A, B) \cong H_{k}(X, B), \forall k \in \mathbb{N}$;

(A.4) se $B$ é um retrato de deformação forte de $A$, então a injeção canônica induz um isomorfismo $H_{k}(X, B) \cong H_{k}(X, A), \forall k \in \mathbb{N}$.

Para uma demonstração dos resultados acima, ver Ramos [33]. Além destes, usaremos também o seguinte fato (ver [11]):

(A.5) a esfera unitária de um espaço normado de dimensão infinita $X, S_{X}$, é contrátil - em outras palavras, $\operatorname{dim} H_{0}\left(S_{X}\right)=1$ e $\operatorname{dim} H_{q}\left(S_{X}\right)=0$, para todo $q \neq 0$.

Definição A.5. O polinômio de Poincarè de um par topológico $(X, A)$ é definido por

$$
\mathcal{P}_{t}(X, A)=\sum_{q \in \mathbb{N}} t^{q} \operatorname{dim} H_{q}(X, A) .
$$

Poremos, quando $A=\emptyset, \mathcal{P}_{t}(X)=\mathcal{P}_{t}(X, \emptyset)$.

\section{A.2 Teoria de Morse para funcionais sobre espaços de Hilbert e o índice de Morse}

Sejam $E$ um espaço de Hilbert e $f: E \rightarrow \mathbb{R}$ um funcional de classe $C^{2}(E, \mathbb{R})$. Denotaremos por $\mathcal{K}_{f}$ o conjunto dos pontos críticos de $f$.

Definição A.6. Sejam $v \in \mathcal{K}_{f}$ e $H_{f}(v)$ a hessiana de $f$ em $v$. Dizemos que $u$ é um ponto crítico não degenerado se existem uma decomposição de $E, E=E^{+} \oplus E^{-}$, e $\nu>0$ satisfazendo

(i) $H_{f}(v)(u, u) \geq \nu\|u\|_{E}^{2}, \forall u \in E^{+}$; 
(ii) $H_{f}(v)(u, u) \leq-\nu\|u\|_{E}^{2}, \forall u \in E^{-}$.

Definição A.7. Seja $v \in \mathcal{K}_{f}$ não degenerado. O índice de Morse de $v, m(v, f)$, é a dimensão do subespaço $E^{-}$da Definição A.6.

Antes de continuarmos, precisamos dar algumas noções sobre a família $\mathbb{S}$ de séries formais em uma variável $t$ com coeficientes em $\mathbb{N} \cup\{+\infty\}$, isto é, $\sum_{k \in \mathbb{N}} a_{k} t^{k}, a_{k} \in \cup\{+\infty\}$.

Para $\mathcal{P} \in \mathbb{S}$, denotamos o $k$-ésimo coeficiente de $\mathcal{P}$ por $c_{k}$, isto é,

$$
c_{k}(\mathcal{P})=a_{k} \Longleftrightarrow \mathcal{P}(t)=\sum_{k} a_{k} t^{k}
$$

Definimos a noção de limite em $\mathbb{S}$ da seguinte forma:

$$
\mathcal{R}=\lim _{n \rightarrow \infty} \mathcal{P}_{n} \Longleftrightarrow c_{k}\left(\mathcal{P}_{n}\right) \rightarrow c_{k}(\mathcal{R}), \text { quando } n \rightarrow \infty \text {, para todo } k \in \mathbb{N} \text {. }
$$

Se identificarmos a série formal $\sum_{k} a_{k} t^{k}$ com a sequência $\left(a_{k}\right)_{k}$, a topologia induzida por (A.1) é equivalente à topologia produto $\prod_{j=0}^{\infty} X_{j}$, onde $X_{j}=\mathbb{N} \cup\{+\infty\}$. Logo, pelo Teorema de Tychonoff, $\mathbb{S}$ é compacto. Se $\mathbb{A} \subset \mathbb{S}$, denotamos por $\overline{\mathbb{A}}$ o fecho de $\mathbb{A}$, isto é,

$$
\overline{\mathbb{A}}=\left\{\mathcal{P} \in \mathbb{S} ; \exists\left(\mathcal{P}_{n}\right)_{n} \subset \mathbb{S} \text { tal que } \mathcal{P}=\lim _{n \rightarrow \infty} \mathcal{P}_{n}\right\}
$$

Definimos, então, os supremo e ínfimo em $\mathbb{S}$ da seguinte forma:

Definição A.8. Se $\mathbb{A} \subset \mathbb{S}$, pomos $\mathcal{R}=\inf \mathbb{A}$, se $\mathcal{R}=\min \overline{\mathbb{A}}$ e $\mathcal{R}=\sup \mathbb{A}$, se $\mathcal{R}=\max \overline{\mathbb{A}}$. Vale ressaltar que inf e sup existem e são únicos [8, Theorem I.1.11].

Definição A.9. Seja $\Lambda$ um conjunto aberto de $E$. Uma função $f \in C^{1}(\bar{\Lambda})$ é chamada função de Morse (generalizada) se

- os pontos críticos de $f$ são todos não degenerados;

- $f$ satisfaz a condição $(P S)$ em $\Lambda$;

- $f$ pode ser estendida a uma função de classe $C^{1}$ em uma vizinhança de $\bar{\Lambda}$.

O conjunto das funções de Morse generalizadas será denotado por $\mathcal{M}(\bar{\Lambda})$.

\section{A.2.1 Blocos de Conley}

Consideremos um campo de vetores $F: E \rightarrow E$ e denotemos por $\eta(t, x)$ a solução do problema de Cauchy

$$
\left\{\begin{array}{l}
\frac{\mathrm{d} \eta}{\mathrm{d} t}=F(\eta) \\
\eta(0, x)=x
\end{array}\right.
$$


Suponhamos que $F$ é tal que o problema anterior é bem posto e, para cada, $x \in E, \eta(t, x)$ está definida, para todo $t \in \mathbb{R}$. Para qualquer conjunto $A \subseteq E$, ponhamos

$$
\begin{aligned}
& W_{+}(A) \equiv W_{+}(F, A)=\bigcap_{t \leq 0} \eta(t, A)=\{x \in A ; \eta(t, x) \in A, \text { para todo } t \geq 0\} ; \\
& W_{-}(A) \equiv W_{-}(F, A)=\bigcap_{t \geq 0} \eta(t, A)=\{x \in A ; \eta(t, x) \in A, \text { para todo } t \leq 0\} ; \\
& G(A) \equiv G(A, F)=W_{+}(A, F) \cap W_{-}(A, F)=\{x \in A ; \eta(t, x) \in A, \text { para todo } t \in \mathbb{R}\} .
\end{aligned}
$$

Além disso, para qualquer $A \subset E$ e $T \geq 0$, pomos

$$
\begin{aligned}
& W_{+}^{T}(A) \equiv W_{+}^{T}(F, A)=\bigcap_{t \in[-T, 0]} \eta(t, A) ; \\
& W_{-}^{T}(A) \equiv W_{-}^{T}(F, A)=\bigcap_{t \in[0, T]} \eta(t, A) ; \\
& G^{T}(A) \equiv G^{T}(F, A)=W_{+}^{T}(A) \cap W_{-}^{T}(A) .
\end{aligned}
$$

Definição A.10. Dado $A \subset E$ fechado, definimos

$$
\Gamma(A)=\left\{x \in \partial A ; \forall \epsilon_{0} \geq 0, \exists \epsilon \in\left(0, \epsilon_{0}\right) \text { tal que } \eta(\epsilon, x) \notin A\right\} .
$$

A este conjunto chamamos conjunto de saída (relativo a $A$ ) e seus pontos são chamados pontos de saída (relativos a A). Definimos também

$$
\Sigma=\Sigma_{F}=\{N \subset E ; \Gamma(N) \text { é fechado }\}
$$

Um conjunto $A \in \Sigma$ é chamado de bloco de Conley.

Vale ressaltar que, mesmo $A$ sendo fechado, $\Gamma(A)$ pode não ter esta propriedade. Desse modo, a definição de bloco de Conley é plausível.

Definição A.11. Se $N \in \Sigma$, definimos o índice de $N$ como

$$
I_{t}(N)=I_{t}(N, F)=\mathcal{P}_{t}(N, \Gamma(N)) .
$$

Dado $N \in \Sigma$, gostaríamos de ressaltar as seguintes propriedades [8, Theorem I.3.8]:

- $I_{t}(N)=\mathcal{P}_{t}\left(N, N \backslash W_{+}(N)\right)$;

- se para algum $T>0, G^{T}(N) \subset \operatorname{int} N$, então

$$
I_{t}(N)=\mathcal{P}_{t}\left(\operatorname{int} N,(\operatorname{int} N) \backslash W_{+}(N)\right),
$$

onde int $N$ denota o interior de $N$ quando $N$ é o fecho de um aberto de $E$, e caso $N=\bar{M}, M$ uma subvariedade de $E, \operatorname{int} N$ denota a própria variedade $M$. 


\section{A.2.2 Índice de Morse generalizado}

Seja a classe de funções

$$
\mathcal{M}_{f}^{\epsilon}(\bar{\Lambda})=\left\{g \in \mathcal{M}(\bar{\Lambda}) ; g(x)=f(x), \text { para } x \notin\left(\mathcal{K}_{f}(\bar{\Lambda})\right)_{\epsilon}\right\},
$$

onde, para $C \subset E,(C)_{\epsilon}$ denota uma $\epsilon$-vizinhança de $C$. Definimos, então

$$
\mathcal{F}(\bar{\Lambda})=\left\{f \in C^{1}(\bar{\Lambda}) ; \mathcal{M}_{f}^{\epsilon}(\bar{\Lambda}) \neq \emptyset, \text { para todo } \epsilon>0\right\} .
$$

Chamamos a atenção para o fato de que se $\Lambda \subset E$ é limitado e $E$ tem dimensão finita, então $\mathcal{F}(\bar{\Lambda})=C^{1}(\bar{\Lambda})$.

Antes de apresentarmos um resultado sobre a classe de funções definida acima, lembremos a definição de operador de Fredholm e indice de Fredholm.

Definição A.12. Seja $E$ espaços de Hilbert. Um operador limitado $T: E \rightarrow E$ é dito de Fredholm se o núcleo $\operatorname{ker} T$ e o conúcleo coker $T:=\operatorname{ker}\left(T^{*}\right)=(\operatorname{im} T)^{\perp}$ têm dimensão finita. O indice de Fredholm de T é o valor

$$
\operatorname{dim} \operatorname{ker} T-\operatorname{dim} \operatorname{coker} T \text {. }
$$

Teorema A.13. ([8, Example I.5.1]) Seja $f$ de classe $C^{2}$ em uma vizinhança de $\bar{\Lambda}$ and satisfazendo a condição $(P S)$. Suponhamos que, para qualquer ponto crítico não degenerado $x \in \mathcal{K}_{f}(\bar{\Lambda})$, o operador linear associado à Hessiana $H_{f}(x)$ seja um operador de Fredholm de indice 0 . Então $f \in \mathcal{F}(\bar{\Lambda})$.

Definição A.14. Um conjunto compacto $\mathcal{K} \subset \mathcal{K}_{f}$ é dito conjunto crítico isolado se existe um conjunto aberto $A$ tal que $\mathcal{K}=A \cap \mathcal{K}_{f}$. Seja $\mathcal{K}$ um conjunto crítico isolado de $f$.

Definimos

$$
i_{t}(\mathcal{K}, f) \equiv i_{t}(\mathcal{K}, f, \omega)=\sup _{\epsilon>0}\left(\inf _{g \in \mathcal{M}_{f}^{\epsilon}(\bar{\omega})} m_{t}\left(\mathcal{K}_{g}(\bar{\omega}), g\right)\right) .
$$

A série formal $i_{t}(\mathcal{K}, f)$ é chamada indice de Morse (generalizado) de $\mathcal{K}$.

Definição A.15. Se $v \in \mathcal{K}_{f}(\bar{\Lambda})$ é um ponto crítico isolado, chamamos de multiplicidade de $v$ o número inteiro $i_{1}(\{v\}, f)$.

Sobre o índice $i_{t}$, temos as seguintes propriedades ([8, Theorem I.5.8]): seja $f \in \mathcal{F}(\bar{\Lambda})$.

- Se $x_{0}$ é um ponto críticos não degenerado de $f$,

$$
i_{t}\left(\left\{x_{0}\right\}\right)=t^{m\left(x_{0}, f\right)},
$$

em particular, $i_{1}\left(\left\{x_{0}\right\}\right)=1^{m\left(x_{0}, f\right)}=1$, isto é, sua multiplicidade é 1 ;

- Se $\mathcal{K}_{1}, \mathcal{K}_{2} \subset \mathcal{K}_{f}$ são conjuntos críticos isolados, então

$$
i_{t}\left(\mathcal{K}_{1} \cup \mathcal{K}_{2}, f\right)=i_{t}\left(\mathcal{K}_{1}, f\right)+i_{t}\left(\mathcal{K}_{2}, f\right) .
$$


- Se $\mathcal{K}$ é discreto, então

$$
i_{t}(\mathcal{K}, f)=\sum_{x \in \mathcal{K}} i_{t}(x, f)
$$

Antes de apresentarmos o teorema mais importante para os nossos estudos, vejamos um resultado auxiliar.

Teorema A.16. (de deformação [8, Theorem I.4.3]) Seja $f \in \mathcal{M}(\bar{\Lambda})$. Existe um campo de vetores pseudo-gradiente $-F$ tal que o problema de Cauchy

$$
\left\{\begin{array}{l}
\frac{\mathrm{d} \eta}{\mathrm{d} t}=F(\eta), \\
\eta(0, v)=v
\end{array}\right.
$$

é bem posto, $\eta(t, v)$ é definida para todo $v \in E$ e $t \in \mathbb{R} e$

$$
\frac{\mathrm{d}}{\mathrm{d} t}(f \circ \eta(t, v))<0 \text { para qualquer } v \notin \mathcal{K}_{f}, t \in \mathbb{R} .
$$

Teorema A.17. ([8, Theorem I.5.9]) Assuma que $f \in \mathcal{F}(\bar{\Lambda})$ é limitada inferiormente em $\Lambda$. Se $F$ é o campo de vetores como no teorema anterior e $\bar{\Lambda} \in \Sigma$, então

$$
i_{t}\left(\mathcal{K}_{f}, f\right)=I_{t}(\bar{\Lambda}, F)+(1+t) \mathcal{Q}(t)
$$

onde $\mathcal{Q} \in \mathbb{S}$.

\section{A.3 Teoria de Morse e multiplicidade e soluções}

Aqui exporemos os resultados relacionados à obtenção da contagem de soluções para os problemas tratados nesta tese. Com este objetivo, fixemos nossas notações. Sejam $E$ um espaço de Hilbert real munido do produto interno $\langle,\rangle_{E}: E \times E \rightarrow \mathbb{R}$, com norma induzida $\|\cdot\|_{E}^{2}=\langle,\rangle_{E}, I: E \rightarrow \mathbb{R}$ um funcional de classe $C^{2}(E, \mathbb{R}), M=\left\{u \in E \backslash\{0\} ; I^{\prime}(u) u=0\right\}$ a variedade de Nehari associada a $I, b=\inf _{M} I$. Seja também $b^{*}>b$ um valor regular de $I$ e definamos os conjuntos $I^{a}=\{u \in E ; I(u) \leq a\}, M^{a}=M \cap I^{a}$, para $a \in \mathbb{R}$.

Suponhamos que:

(i) $I=\frac{1}{2}\|\cdot\|_{E}^{2}-\Psi$, onde $\Psi: E \rightarrow \mathbb{R}$ é tal que $\Psi(0)=0$ e $t>0 \mapsto \Psi^{\prime}(t u) u / t$ é crescente e ilimitado superiormente, para todo $u \in E \backslash\{0\}$;

(ii) I satisfaz a condição $(P S)_{c}$ para todo $c \in \mathbb{R}$, e dado $u \in E$, existe um operador auto-adjunto $L(u): E \rightarrow E$ tal que $H_{I}(u)(v, v)=\langle L(u) v, v\rangle_{E}$, para todo $v \in E$;

(iii) $M$ é difeomorfo à esfera unitária de $E$;

(iv) existe $\delta_{0}>0$ tal que $\inf _{M} I \geq \delta_{0}$; 
$(v)$ existem um conjunto não vazio $\Theta \subset \mathbb{R}^{N}$ e aplicações $\Phi: \Theta^{-} \rightarrow M^{b^{*}}, \beta: M^{b^{*}} \rightarrow \Theta^{+}$ tais que $\beta \circ \Phi=I d_{\Theta^{-}}$, onde $\Theta^{+}=\left\{x \in \mathbb{R}^{N} ; \operatorname{dist}(x, \Theta) \leq r\right\}$,

$\Theta^{-}=\{x \in \Theta ; \operatorname{dist}(x, \partial \Theta) \geq r\}, r>0$ tomado de modo que $\Theta, \Theta^{+}$e $\Theta^{-}$são homotopicamente equivalentes.

As hipóteses requeridas são todas satisfeitas pelos respectivos entes presentes nos capítulos deste trabalho.

O primeiro resultado mostra como o conjunto $\Theta$ relaciona-se com o conjunto $M^{b^{*}}$.

Lema A.18. Sob as hipóteses e notações acima, temos

$$
\mathcal{P}_{t}\left(M^{b^{*}}\right)=\mathcal{P}_{t}(\Theta)+\mathcal{Q}(t)
$$

onde $\mathcal{Q}$ é um polinômio com coeficientes não-negativos.

\section{Demonstração.}

Observe que a aplicação $\Phi$ induz o homeomorfismo $(\Phi)_{k}$ entre os grupos de homologia de dimensão $k, k \in \mathbb{N}$,

$$
H_{k}\left(\Theta^{-}\right) \stackrel{(\Phi) k}{\rightarrow} H_{k}\left(M^{b^{*}}\right) .
$$

Mais ainda, $(\Phi)_{k}$ é injetor, uma vez que $\Phi$ o é. Logo $(\Phi)_{k}$ é um isomorfismo sobre sua imagem, donde

$$
\operatorname{dim} H_{k}\left(\Theta^{-}\right) \leq \operatorname{dim} H_{k}\left(M^{b^{*}}\right) .
$$

Portanto

$$
\begin{aligned}
\mathcal{P}_{t}\left(M^{b^{*}}\right) & =\sum_{k \in \mathbb{N}} \operatorname{dim} H_{k}\left(M^{b^{*}}\right) t^{k}=\sum_{k \in \mathbb{N}} \operatorname{dim} H_{k}\left(\Theta^{-}\right) t^{k}+\mathcal{Q}(t) \\
& =\mathcal{P}_{t}\left(\Theta^{-}\right)+\mathcal{Q}(t)
\end{aligned}
$$

onde $\mathcal{Q}$ é um polinômio com coeficientes não-negativos.

Por fim, como $\Theta$ e $\Theta^{-}$são homotopicamente equivalentes,

$$
H_{k}(\Theta) \cong H_{k}\left(\Theta^{-}\right), \forall k \in \mathbb{N},
$$

donde

$$
\mathcal{P}_{t}\left(M^{b^{*}}\right)=\mathcal{P}_{t}(\Theta)+\mathcal{Q}(t)
$$

Dado $a \in \mathbb{R}$, definamos o conjunto $I^{a}=\{u \in E ; I(u) \leq a\}$.

Lema A.19. Sejam $\delta \in\left(0, \delta_{0}\right), \delta_{0}$ fixado no início desta seção, e a $\in(\delta, \infty]$ um valor regular. Temos

$$
\mathcal{P}_{t}\left(I^{a}, I^{\delta}\right)=t \mathcal{P}_{t}\left(M^{a}\right)
$$

Para mostrarmos este lema, precisamos de alguns resultados. 
Lema A.20. Se $d<a, d<b=\inf _{M} I$, são valores regulares, então

$$
\mathcal{P}_{t}\left(I^{a}, I^{d}\right)=P_{t}\left(\overline{\Sigma(d, a)}, I^{-1}(\{d\})\right),
$$

onde $\Sigma(d, a):=\{u \in E ; d<I<a\}$.

\section{Demonstração.}

Mostremos que

$$
H_{k}\left(I^{a}, I^{d}\right)=H_{k}\left(\overline{\Sigma(d, a)}, I^{-1}(\{d\})\right), \forall k \in \mathbb{N} .
$$

Para isto, usaremos as propriedades $(A .3)$ e $(A .4)$.

Sejam os conjuntos

$$
A:=\{u \in E ; t(u)>1\} \quad \text { e } \quad B:=\{u \in E ; t(u)<1\}
$$

onde, para cada $u \in E \backslash\{0\}, t(u)>0$ é o (único) número tal que $t(u) u \in M$.

Primeiramente, vejamos que, para $\alpha \geq 0$ suficientemente pequeno,

$$
I^{-1}(\{d\}) \cap B \text { é um retrado de deformação forte de } I^{d+\alpha} \cap B \text {. }
$$

Com efeito, definamos, para cada $u \in I^{d+\alpha} \cap B, \xi(u)>0$ como sendo o valor tal que

$$
I(\xi(u) u)=d .
$$

Vale ressaltar que, pela hipótese sobre $\Psi$ dada no início deste apêndice, $\xi(u)$ é único. Notemos que $\xi$ é contínua. De fato, seja $G:(0, \infty) \times\left(I^{d+\alpha} \cap B\right) \rightarrow \mathbb{R}$ dada por

$$
G(\xi, u)=I(\xi u)-d
$$

Sejam $\xi_{0}>0$ e $u_{0} \in I^{d+\alpha} \cap B$ tais que $G\left(\xi_{0}, u_{0}\right)=0$. Logo $I\left(\xi_{0} u_{0}\right)=d<b$ e, consequentemente, $\xi_{0} u_{0} \notin M$, donde

$$
\frac{\partial G}{\partial t}\left(\xi_{0}, u_{0}\right)=I^{\prime}\left(\xi_{0} u_{0}\right) u_{0} \neq 0 .
$$

Assim, pelo Teorema da Função Implícita, $\xi$ é contínua em $I^{d+\alpha} \cap B$.

Consideremos a aplicação

$$
\begin{aligned}
H:[0,1] \times I^{d+\alpha} \cap B & \rightarrow I^{d+\alpha} \cap B \\
(t, u) & \mapsto H(t, u):=(1-t+t \xi(u)) u
\end{aligned}
$$

Então, para todo $u \in I^{d+\alpha} \cap B$ :

- $H$ é contínua e $H(t, u) \in I^{d+\alpha} \cap B, \forall t \in[0,1]$, isto é, $H$ está bem definida;

- $H(0, u)=u$;

- $I(H(1, u))=d$; 
- $H(t, u)=u, \forall u \in I^{-1}(\{d\}) \cap B, \forall t \in[0,1]$.

Portanto, lembrando a Definição A.4, (A.2) é válido.

Analogamente, mostramos que, para $\alpha \geq 0$ suficientemente pequeno,

$$
I^{-1}(\{d\}) \cap A \text { é um retrato de deformação forte de } \overline{\Sigma(d, d+\alpha)} \cap A
$$

usando a aplicação

$$
\begin{aligned}
\sigma: \overline{\Sigma(d, d+\alpha)} \cap A & \rightarrow \mathbb{R} \\
u & \mapsto \sigma(u) \in(0,1] \text { tal que } I(\sigma(u) u)=d .
\end{aligned}
$$

Com as aplicações acima, definamos

$$
\begin{aligned}
\tilde{\xi}:(\overline{\Sigma(d, d+\alpha)} \cap A) \cup\left(I^{d+\alpha} \cap B\right) & \rightarrow \mathbb{R} \\
u & \mapsto \tilde{\xi}= \begin{cases}\sigma(u), & u \in \overline{\Sigma(d, d+\alpha)} \cap A \\
\xi(u), & u \in I^{d+\alpha} \cap B,\end{cases}
\end{aligned}
$$

e, como antes,

$$
I^{-1}(\{d\}) \text { é um retrato de deformação forte de }(\overline{\Sigma(d, d+\alpha)} \cap A) \cup\left(I^{d+\alpha} \cap B\right)
$$

Temos, também, com $\tilde{\sigma}: I^{d+\alpha} \rightarrow \mathbb{R}$ dada por

$$
\tilde{\sigma}(u):= \begin{cases}\sigma(u), & u \in \overline{\Sigma(d, d+\alpha)} \cap A, \\ \bar{\sigma}(u), & u \in \overline{\Sigma(d, d+\alpha)} \cap B, \\ 1, & u \notin \overline{\Sigma(d, d+\alpha)},\end{cases}
$$

onde $\bar{\sigma}(u) \geq 1$ é o análogo de $\sigma$ para $u \in \overline{\Sigma(d, d+\alpha)} \cap B$,

$$
I^{d} \text { é um retrato de deformação forte de } I^{d+\alpha},
$$

para $\alpha \geq 0$ suficientemente pequeno, e

$$
\overline{\Sigma(d, a)} \text { é um retrato de deformação forte de } I^{a} \backslash\left(\operatorname{int}\left(I^{d} \cap A\right)\right) \text {, }
$$

usando $\bar{\xi}: I^{a} \backslash\left(\operatorname{int}\left(I_{\lambda}^{d} \cap A\right)\right) \rightarrow \mathbb{R}$ dada por

$$
\bar{\xi}(u):= \begin{cases}\xi(u), & u \in I^{d} \cap B, \\ 1, & u \in \overline{\Sigma(d, a)} .\end{cases}
$$

Com os retratos listados, temos, para todo $k \in \mathbb{N}$ :

- $\operatorname{por}($ A.4),

$$
H_{k}\left(I^{a}, I^{d}\right)=H_{k}\left(I^{a}, I^{d+\alpha}\right)
$$


- pela propriedade da excisão e pelo fato $\operatorname{de} \overline{\operatorname{int}\left(I^{d} \cap A\right)} \subset \operatorname{int}\left(I^{d+\alpha} \cap A\right)$,

$$
H_{k}\left(I^{a}, I^{d+\alpha}\right)=H_{k}\left(I^{a} \backslash \operatorname{int}\left(I^{d} \cap A\right), I^{d+\alpha} \backslash \operatorname{int}\left(I^{d} \cap A\right)\right) ;
$$

- tendo em vista que $I^{d+\alpha} \backslash \operatorname{int}\left(I^{d} \cap A\right)=(\overline{\Sigma(d, d+\alpha)} \cap A) \cup\left(I^{d+\alpha} \cap B\right)$,

$$
\begin{aligned}
H_{k}\left(I^{a} \backslash \operatorname{int}\left(I^{d} \cap A\right), I^{d+\alpha} \backslash \operatorname{int}\left(I^{d} \cap A\right)\right) & \\
\quad= & H_{k}\left(I^{a} \backslash \operatorname{int}\left(I^{d} \cap A\right),(\overline{\Sigma(d, d+\alpha)} \cap A) \cup\left(I^{d+\alpha} \cap B\right)\right) ;
\end{aligned}
$$

- $\operatorname{por}(\mathrm{A} .3)$,

$$
\begin{aligned}
H_{k}\left(I^{a} \backslash \operatorname{int}\left(I^{d} \cap A\right),(\overline{\Sigma(d, d+\alpha)} \cap A) \cup\left(I^{d+\alpha} \cap B\right)\right) & = \\
& =H_{k}\left(I^{a} \backslash \operatorname{int}\left(I^{d} \cap A\right), I^{-1}(\{d\})\right) ;
\end{aligned}
$$

- $\operatorname{por}(\mathrm{A} .5)$,

$$
H_{k}\left(I^{a} \backslash \operatorname{int}\left(I^{d} \cap A\right), I^{-1}(\{d\})\right)=H_{k}\left(\overline{\Sigma(d, a)}, I^{-1}(\{d\})\right) ;
$$

Então

$$
H_{k}\left(I^{a}, I^{d}\right)=H_{k}\left(\overline{\Sigma(d, a)}, I^{-1}(\{d\})\right), \quad \forall k \in \mathbb{N},
$$

e daí

$$
\mathcal{P}_{t}\left(I^{a}, I^{d}\right)=\mathcal{P}_{t}\left(\overline{\Sigma(d, a)}, I^{-1}(\{d\})\right)
$$

como queríamos.

No próximo resultado, invocaremos as definições mencionadas na Seção A.2.1 deste apêndice. Dito isto, sejam $d, a$ valores regulares de $I, d<b<a$, e $\sigma>0$ tal que $d+3 \sigma<b$ e consideremos a aplicação $J: E \rightarrow \mathbb{R}$ dada por $J(u)=\nabla I(u) u$. Sejam também os conjuntos

$$
U=J^{-1}((-\sigma, \sigma)) \cap I^{-1}((d+2 \sigma,+\infty)), \quad V=J^{-1}((-2 \sigma, 2 \sigma)) \cap I^{-1}((d+\sigma,+\infty))
$$

e a aplicação $\chi: E \rightarrow \mathbb{R}$ dada por

$$
\chi(u)=\frac{\operatorname{dist}(u, E \backslash V)}{\operatorname{dist}(u, E \backslash V)+\operatorname{dist}(u, U)} .
$$

Desta forma, o campo de vetores

$$
F(u)=-\nabla I(u)+\chi(u) \frac{\langle\nabla I(u), \nabla J(u)\rangle_{E}}{\|\nabla J(u)\|_{E}^{2}} \nabla J(u)
$$

é localmente lipschitziana. Além disso, $F$ é tangente a cada curva de nível de $J$ contida em $U$, em particular, a $M=J^{-1}(0)$. 
Lema A.21. Sejam $d<a, d<b=\inf _{M} I$, valores regulares e $F$ o campo dado em (A.6). Então $\overline{\Sigma(d, a)}$ é um bloco de Conley e

$$
\Gamma(\overline{\Sigma(d, a)})=I^{-1}(\{d\}) \text { e } W_{+}(\overline{\Sigma(d, a)})=\overline{\Sigma(d, a)} \cap M=M^{a} .
$$

\section{Demonstração.}

Seja $\eta: \mathbb{R} \times E \rightarrow E$ o fluxo associado à EDO

$$
\left\{\begin{array}{l}
\frac{\mathrm{d} \eta}{\mathrm{d} t}(t, u)=\frac{F(\eta)}{1+\|F(\eta)\|_{E}} \\
\eta(0, u)=u
\end{array}\right.
$$

Notemos que, para qualquer $u \in E$ e todo $t \in \mathbb{R}$,

$$
\begin{aligned}
\frac{\mathrm{d}}{\mathrm{d} t} I(\eta(t, u)) & =\left[-\|\nabla I(\eta(t, u))\|_{E}^{2}+\chi(\eta(t, u)) \frac{\langle\nabla I(\eta(t, u)), \nabla J(\eta(t, u))\rangle_{E}^{2}}{\|\nabla J(\eta(t, u))\|_{E}^{2}}\right] \frac{1}{1+\|F(\eta(t, u))\|_{E}} \\
& \leq\left[\|\nabla I(\eta(t, u))\|_{E}^{2}(\chi(\eta(t, u))-1)\right] \frac{1}{1+\|F(\eta(t, u))\|_{E}} \\
& \leq 0 .
\end{aligned}
$$

Além disso, se $u \in I^{-1}(\{d, a\})=\partial \Sigma(d, a)$, pela natureza de $a$ e $d$ e pela forma como a vizinhança $V$ foi tomada,

$$
\left.\frac{\mathrm{d}}{\mathrm{d} t} I(\eta(t, u))\right|_{t=0}<0 .
$$

Portanto, tendo em vista a continuidade do fluxo $\eta$,

$$
\Gamma(\overline{\Sigma(d, a)})=I^{-1}(\{d\}) .
$$

Daí segue pela Definição A.10 que $\overline{\Sigma(d, a)}$ é um bloco de Conley. Pelo fato de o campo ser tangente a $M$ e por (A.7), temos $W_{+}(\overline{\Sigma(d, a)})=M^{a}$.

Corolário A.22. Sob as condições e notações do Lema A.21,

$$
\mathcal{P}_{t}\left(\overline{\Sigma(d, a)}, I^{-1}(\{d\})\right)=\mathcal{P}_{t}(\Sigma(d, a), \Sigma(d, a) \backslash M) .
$$

\section{Demonstração.}

Com efeito, como $d, a$ são valores regulares e o fluxo $\eta$ é contínuo, temos, para $T>0$ suficientemente pequeno,

$$
G^{T}(\overline{\Sigma(d, a)}) \subset \Sigma(d, a) .
$$

Logo o resultado segue do Lema A.21, Definição A.11 e das propriedades do índice de blocos de Conley. 
Sobre a demonstração acima, vale ressaltar que podemos concluir que

$$
H_{k}\left(\overline{\Sigma(d, a)}, I^{-1}(\{d\})=H_{k}(\overline{\Sigma(d, a)}, \overline{\Sigma(d, a)} \backslash M)\right.
$$

usando argumentos semelhantes aos usados no Lema A.20.

Para a demonstração do próximo resultado, ver [24, Corollary 11.14].

Lema A.23. Sejam $\mathcal{M}$ uma variedade e $\mathcal{N} \subset \mathcal{M}$ uma subvariedade fechada e orientável de codimensão d. Se $W \subset \mathcal{N}$ é fechado em $\mathcal{N}$, então

$$
\mathcal{P}_{t}(\mathcal{M}, \mathcal{M} \backslash W)=t^{d} \mathcal{P}_{t}(\mathcal{N}, \mathcal{N} \backslash W)
$$

\section{Demonstração do Lema A.19.}

Basta aplicar o Lema A.23 $\operatorname{com} \mathcal{M}=\overline{\Sigma(d, a)}$ e $\mathcal{N}=W=\overline{\Sigma(d, a)} \cap M$. Logo

$$
\begin{aligned}
\mathcal{P}_{t}(\overline{\Sigma(d, a)}, \overline{\Sigma(d, a)} \backslash M) & =t \mathcal{P}_{t}(\overline{\Sigma(d, a)} \cap M, \emptyset) \\
& =t \mathcal{P}_{t}\left(M^{a}\right),
\end{aligned}
$$

uma vez que $M$ é difeormorfo à esfera unitária em $E$. A conclusão segue dos Lema A.20 e Corolário A.22 tomando $d=\delta$.

Corolário A.24. Sejam $\delta$ como no Lema A.19. Temos

$$
\mathcal{P}_{t}\left(I^{b^{*}}, I^{\delta}\right)=t \mathcal{P}_{t}(\Theta)+t \mathcal{Q}(t)
$$

$e$

$$
\mathcal{P}_{t}\left(E, I^{\delta}\right)=t \mathcal{P}_{t}(M)=t,
$$

onde $\mathcal{Q}$ é um polinômio com coeficientes não-negativos.

\section{Demonstração.}

Notemos que $b^{*}$ é um valor regular de $I$. Então (A.8) segue dos Lemas A.18 e A.19 com $a=b^{*}$. A igualdade (A.9) segue do Lema A.19 com $a=\infty$ e do fato de $M$ ser difeomorfa à esfera unitária de $E$ com (A.5).

Lema A.25. Seja $\delta$ como no Lema A.19. Temos

$$
\mathcal{P}_{t}\left(E, I^{b^{*}}\right)=t^{2}\left[P_{t}(\Theta)+\mathcal{Q}(t)-1\right],
$$

onde $\mathcal{Q}$ é um polinômio com coeficientes não-negativos. 


\section{Demonstração.}

Consideremos $\delta>0$ como no enunciado, e a sequência exata

$$
\begin{aligned}
\ldots \longrightarrow & \longrightarrow H_{k}\left(E, I^{\delta}\right) \stackrel{j_{k}}{\longrightarrow} H_{k}\left(E, I^{b^{*}}\right) \stackrel{\partial_{k}}{\longrightarrow} \\
& \stackrel{\partial_{k}}{\longrightarrow} H_{k-1}\left(I^{b^{*}}, I^{\delta}\right) \stackrel{i_{k-1}}{\longrightarrow} H_{k-1}\left(E, I^{\delta}\right) \longrightarrow \ldots
\end{aligned}
$$

Por (A.9),

$$
\operatorname{dim} H_{k}\left(E, I^{\delta}\right)=0, \forall k \neq 1 .
$$

Logo, da exatidão da sequência acima, $\partial_{k}$ é um isomorfismo, para todo $k \geq 3$, donde,

$$
\operatorname{dim} H_{k}\left(E, I^{b^{*}}\right)=\operatorname{dim} H_{k-1}\left(I^{b^{*}}, I^{\delta}\right), \forall k \geq 3 .
$$

Para $k=2$, temos

$$
\begin{aligned}
\ldots \longrightarrow & H_{2}\left(E, I^{\delta}\right) \stackrel{j_{2}}{\longrightarrow} H_{2}\left(E, I^{b^{*}}\right) \stackrel{\partial_{2}}{\longrightarrow} \\
& \stackrel{\partial_{2}}{\longrightarrow} H_{1}\left(I^{b^{*}}, I^{\delta}\right) \stackrel{i_{1}}{\longrightarrow} H_{1}\left(E, I^{\delta}\right) \longrightarrow \ldots
\end{aligned}
$$

Como $j_{2}$ é sobrejetora, pois é o homomorfismo induzido pela projeção canônica, e $\operatorname{dim} H_{2}\left(E, I^{\delta}\right)=0$, por (A.9), temos

$$
H_{2}\left(E, I^{b^{*}}\right)=j_{2}\left(H_{2}\left(E, I^{\delta}\right)\right)=\{0\} .
$$

Para $k=1$,

$$
\begin{aligned}
\ldots \longrightarrow & H_{1}\left(I^{b^{*}}, I^{\delta}\right) \stackrel{i_{1}}{\longrightarrow} H_{1}\left(E, I^{\delta}\right) \stackrel{j_{1}}{\longrightarrow} \\
& \stackrel{j_{1}}{\longrightarrow} H_{1}\left(E, I^{b^{*}}\right) \stackrel{\partial_{1}}{\longrightarrow} H_{0}\left(I^{b^{*}}, I^{\delta}\right) \longrightarrow \ldots
\end{aligned}
$$

Temos, pelo fato de $E$ ser conexo e (A.2),

$$
H_{0}\left(E, I^{b^{*}}\right)=0 .
$$

Note que $i_{1}$ é um isomorfismo. Com efeito, por (A.1),

$$
H_{0}(\Theta) \neq\{0\} .
$$

Logo, por (A.8),

$$
H_{1}\left(I^{b^{*}}, I^{\delta}\right) \neq\{0\} .
$$

Além disso, por (A.9),

$$
\operatorname{dim} H_{1}\left(E, I^{\delta}\right)=1 .
$$

Portanto, como $i_{1}$ é injetora, já que é o homomorfismo induzido pela injeção canônica, temos

$$
\operatorname{dim} H_{1}\left(I^{b^{*}}, I^{\delta}\right)=1
$$

e, consequentemente, $i_{1}$ é um isomorfismo. 
Agora, da exatidão da sequência, de $i_{1}$ ser isomorfismo e $j_{1}$ ser sobrejetora,

$$
\operatorname{dim} H_{1}\left(E, I^{b^{*}}\right)=0 .
$$

Por fim, pelo Corolário A.24 e (A.10) - (A.15),

$$
\begin{aligned}
\mathcal{P}_{t}\left(E, I^{b^{*}}\right)= & \sum_{k \geq 0} t^{k} \operatorname{dim} H_{k}\left(E, I^{b^{*}}\right) \\
= & \sum_{k \geq 3} t^{k} \operatorname{dim} H_{k}\left(E, I^{b^{*}}\right)+t^{2} \operatorname{dim} H_{2}\left(E, I^{b^{*}}\right) \\
& +t \operatorname{dim} H_{1}\left(E, I^{b^{*}}\right)+\operatorname{dim} H_{0}\left(E, I^{b^{*}}\right) \\
= & t \sum_{k \geq 3} t^{k} \operatorname{dim} H_{k}\left(E, I^{b^{*}}\right)+0+0+0 \\
= & t\left(P_{t}\left(I^{b^{*}}, I^{\delta}\right)-t \operatorname{dim} H_{1}\left(I^{b^{*}}, I^{\delta}\right)-\operatorname{dim} H_{0}\left(I^{b^{*}}, I^{\delta}\right)\right) \\
= & t^{2}\left(\mathcal{P}_{t}(\Omega)+\mathcal{Q}(t)-1\right) .
\end{aligned}
$$

Lema A.26. Suponha que o conjunto $\mathcal{K}_{I}$ de pontos críticos de $I$ é discreto. Então

$$
\sum_{u \in \mathcal{C}_{1}} i_{t}(u)=t \mathcal{P}_{t}(\Theta)+t \mathcal{Q}(t)+(1+t) \mathcal{Q}_{1}(t)
$$

$$
\sum_{u \in \mathcal{C}_{2}} i_{t}(u)=t^{2}\left[\mathcal{P}_{t}(\Theta)+\mathcal{Q}(t)-1\right]+(1+t) \mathcal{Q}_{2}(t)
$$

onde

$$
\mathcal{C}_{1}:=\left\{u \in \mathcal{K} ; \delta<I(u) \leq b^{*}\right\}
$$

$e$

$$
\mathcal{C}_{2}:=\left\{u \in \mathcal{K} ; b^{*}<I(u)\right\}
$$

\section{Demonstração.}

De fato, primeiramente observemos que $\mathcal{C}_{1}, \mathcal{C}_{2}$ são conjuntos críticos isolados de $I$. Logo $i_{t}\left(\mathcal{C}_{1}, I\right)$ e $i_{t}\left(\mathcal{C}_{2}, I\right)$ estão bem definidos.

Supondo que $\left.I\right|_{\overline{\Sigma\left(\delta, b^{*}\right)}} \in \mathcal{F}\left(\overline{\Sigma\left(\delta, b^{*}\right)}\right)$, fato que veremos ao final da demonstração, temos, pelo Teorema A.17,

$$
i_{t}\left(\left.I\right|_{\overline{\Sigma\left(\delta, b^{*}\right)}}\right)=I_{t}\left(\overline{\Sigma\left(\delta, b^{*}\right)}, F\right)+(1+t) \mathcal{Q}_{1}(t),
$$

com $\mathcal{Q}_{1} \in \mathbb{S}$. Além disso, pela Definição A.11 e Lemas A.21 e A.20, com $a=b^{*}, d=\delta$,

$$
i_{t}\left(\left.I\right|_{\overline{\Sigma\left(\delta, b^{*}\right)}}\right)=\mathcal{P}_{t}\left(I^{b^{*}}, I^{\delta}\right)+(1+t) \mathcal{Q}_{1}(t) .
$$

Por fim, pelo Corolário A.24, mais precisamente por (A.8),

$$
i_{t}\left(\left.I\right|_{\overline{\Sigma\left(\delta, b^{*}\right)}}\right)=t \mathcal{P}_{t}(\Theta)+t \mathcal{Q}(t)+(1+t) \mathcal{Q}_{1}(t) .
$$


A igualdade (A.17) segue de forma análoga pelo Lema A.20, com $a=+\infty, d=b^{*}$, e Corolário A.24, (A.9).

Vejamos que $\left.I\right|_{\overline{\Sigma\left(\delta, b^{*}\right)}} \in \mathcal{F}\left(\overline{\Sigma\left(\delta, b^{*}\right)}\right)$. Para isto, usaremos o Teorema A.13. Mostremos, então, que, dado $u \in \overline{\Sigma\left(\delta, b^{*}\right)}$ ponto crítico não degenerado de $I$, o operador linear associado à Hessiana $H_{I}(u)$ tem índice de Fredholm 0. Com efeito, por hipótese, existe $L(u): E \rightarrow E$ auto-adjunto tal que

$$
H_{I}(u)(v, v)=\langle L(u) v, v\rangle_{E}, \quad \forall v \in E .
$$

Dessa forma $\operatorname{dim} \operatorname{ker} L(u)=\operatorname{dim} \operatorname{coker} L(u)$. Resta mostrar, então, que $L(u)$ é um operador de Fredholm, isto é, que ker $L(u)$ te dimensão finita.

Afirmamos que ker $L(u)=\{0\}$. De fato, pela definição de ponto crítico não degenerado, existem $\nu>0$ e uma decomposição de $E$ em soma direta $E=E^{+} \oplus E^{-}$tais que

$$
H_{I}(u)(v, v) \geq \nu\|v\|_{E}^{2}, \forall v \in E^{+} \quad \text { e } \quad H_{I}(u)(v, v) \leq-\nu\|v\|_{E}^{2}, \forall v \in E^{-} .
$$

Seja $w \in \operatorname{ker} L(u)$. Sejam, também, $w^{+} \in E^{+}, w^{-} \in E^{-}$tais que $w=w^{+}+w^{-}$. Observemos que

$$
\begin{aligned}
0 & =\left\langle L(u) w, w^{+}-w^{-}\right\rangle_{E}=\left\langle L(u) w^{+}, w^{+}\right\rangle_{E}-\left\langle L(u) w^{-}, w^{-}\right\rangle_{E} \\
& =H_{I}(u)\left(w^{+}, w^{+}\right)-H_{I}(u)\left(w^{-}, w^{-}\right) \\
& \geq \nu\left(\left\|w^{+}\right\|_{E}^{2}+\left\|w^{-}\right\|_{E}^{2}\right)
\end{aligned}
$$

ou seja, $w=0$. Assim, $\operatorname{ker} L(u)=\{0\}$ e, pelas considerações feitas, $\left.I\right|_{\overline{\Sigma\left(\delta, b^{*}\right)}} \in \mathcal{F}\left(\overline{\Sigma\left(\delta, b^{*}\right)}\right)$. 



\section{Referências Bibliográficas}

[1] ABATANGELO, L.; TERRACINI, S. Solutions to nonlinear Schrödinger equations with singular electromagnetic potential and critical exponent. J. Fixed Point Theory Appl., v. 10, p. 147-180, 2011.

[2] ADAMS, R.; FOURNIER, J. F. Sobolev Spaces. Academic Press, 2 ed., 2003.

[3] ALVES, C. O.; FIGUEIREDO, G. M.; FURTADO, M. F. Multiple solutions for a nonlinear Schrödinger equation with magnetic fields. J. Differential Equations, v. 251, p. 2534-2548, 2011.

[4] AmBrosetti A.; BADiAle, M.; CINGOlAnI, S. Semiclassical states of nonlinear Schrödinger equations. Arch. Rational Mech. Anal., v. 140, p. 285-300, 1997.

[5] AMBROSETTI A.; RABINOWITZ, P. H. Dual variational methods in critcal point theory and applications. J. Funct. Anal., v. 44, p. 349-381, 1973.

[6] AMBROSETTI A.; RUIZ, D. Radial solutions concentrating on spheres of nonlinear Schrödinger equations with vanishing potentials. Proc. Royal Soc. Edinburgh, v. 136 A, p. 889-907, 2006.

[7] BARILE, S. A multiplicity result for singular NLS equations with magnetic potentials. Nonlinear Anal., v. 68, p. 3525-3540, 2008.

[8] BENCI, V. Introduction to Morse theory: a new approach. In: MATZEU, M.; VIGNOLI, A. Topological nonlinear analysis: Degree, Singularity, and Variations. Progr. Nonlinear Differential Equations Appl., 15. Boston: Birkhäuser Boston, 1995. cap. 2, p. 37-177. 
[9] BENCI, V.; CERAMI, G. Multiple positive solutions of some elliptic problems via the Morse theory and the domain topology. Calc. Var. Partial Differential Equations, v. 2, p. 29-48, 1994.

[10] BENCI, V.; CERAMI, G. The effect of the domain topology on the number of positive solutions of nonlinear elliptic problems. Arch. Rational Mech. Anal., v. 114, p. 79-93, 1991.

[11] BENYAMINI, Y.; STERNFELD, Y. Sphere in infinite-dimensional normed spaces are lipschitz contractible. Proc. Amer. Math. Soc., v. 88, n. 3, 1983.

[12] BERESTYCKI, H.; LIONS, P.L. Nonlinear scalar field equations, I. Arch. Ration. Mech. Anal., v. 82, p. 313-346, 1983.

[13] BREZIS, H. Analyse Fonctionnelle: Théorie et applications. Collection Mathématiques Appliquées pour la Maîtrise. Paris: Masson, 1983.

[14] CANDELA, A. M.; LAZZO, M. Positive solutions for a mixed boundary problem. Nonlinear Anal., v. 24, p. 1109-1117, 1995.

[15] CAO, D.; TANG, Z. Existence and uniqueness of multi-bump bound states of nonlinear Schrödinger equations with electromagnetic fields. J. Differential Equations, v. 222, p. 381-424, 2006.

[16] CHABROWSKI, J. AND A. SZULKIN On the Schrödinger equation involving a critical Sobolev exponent and magnetic field, Top. Meth. Nonlinear Anal., v. 25, p. 3-21, 2005.

[17] CINGOLANI, S. Semiclassical stationary states of Nonlinear Schrödinger equations with an external magnetic field. J. Differential Equations, v. 188, p. 52-79, 2003.

[18] CINGOLANI, S.; CLAPP M. Intertwining semiclassical bound states to a nonlinear magnetic Schrödinger equation. Nonlinearity, v. 22, p. 2309-2331, 2009.

[19] CINGOLANI, S. JEANJEAN; L.; SECCHI, S. Multi-peak solutions for magnetic NLS equations without non-degeneracy conditions. ESAIM Control Optim. Calc. Var., v. 15, p. 653-675, 2009.

[20] CINGOLANI, S. JEANJEAN; L.; SECCHI, S. Semiclassical limit for nonlinear Schrödinger equations with electromagnetic fields. J. Math. Anal. Appl., v. 275, p. 108-130, 2002.

[21] CINGOLANI, S.; SECCHI, S. Semiclassical states for NLS equations with magnetic potentials having polynomial growths. J. Math. Phys., v. 46, n. 5, p. 1-19, 2005. 
[22] CINGOLANI, S.; VANNELLA, G. Multiple positive solutions for a critical quasilinear equation via Morse theory. Ann. Inst. H. Poincaré Anal. Non Linéaire, v. 26, p. 397-413, 2009.

[23] DEL PINO, M.; FELMER, P. Local mountain pass for semilinear elliptic problems in unbounded domains. Calc. Var. Partial Differential Equations, v. 4, p. 121-137, 1996.

[24] DOLD, A. Lectures on Algebraic Topology. Springer-Verlag, 2 ed., 1980.

[25] EKeland, I. On the variational principle, J. Math. Anal. Appl., v. 47, p. 324-353, 1974.

[26] ESTEBAN, J. M.; LIONS, P. L. Stationary solutions of nonlinear Schrödinger equations with an external magnetic field. In: COLOMBINI, F. et al. PDE and Calculus of Variations. Progr. Nonlinear Differential Equations Appli., v. 1, Boston: Birkhäuser, 1989, p. 401-449.

[27] FLOER, L. A.; WEINSTEIN, A. Nonspreading wave packets for the cubic Schrödinger equation with a bounded potential. J. Funct. Anal., v. 69, p. 397-408, 1986.

[28] KURATA, K. Existence and semi-classical limit of the least energy solution to a nonlinear Schrödinger equation with electromagnetic fields. Nonlinear Anal., v. 62, p. 615-628, 2005.

[29] LI, G.; PENG, S.; WANG, C. Infinitely many solutions for nonlinear Schrödinger equations with electromagnetic fields. J. Differential Equations, v. 251, p. 3500-3521, 2011.

[30] LIANG, S.; ZHANG, J. Solutions of perturbed Schrödinger equations with electromagnetic fields and critical nonlinearity. Proc. Edinb. Math. Soc., v. 54, p. 131-147, 2011.

[31] LIONS, P. L. The concentration-compactness principle in the calculus of variations. The locally compact case, Part 2. Ann. Inst. H. Poincaré Anal. Non Linéaire, v. 1, p. 223-283, 1984.

[32] RABINOWITZ, P. H. On a class of nonlinear Schrödinger equations. Z. Angew Math. Phys., v. 43, n. 2, p. 270-291, 1992.

[33] RAmOS, M. Teoremas de Enlace na Teoria dos Pontos Críticos. Lisboa: Faculdade de Ciências da Universidade de Lisboa, v. 2, 1993.

[34] SQUASSINA, M. Soliton dynamics for the nonlinear Schrödinger equation with magnetic field. Manuscripta Math., 130, p. 461-494, 2009. 
[35] W. A. STRAUSS, Existence of solitary waves in higher dimensions. Comm. Math. Phys., v. 55, n. 2, p. 149-162, 1977.

[36] SUlEM, C.; SUlEM, P. L. The Nonlinear Schrödinger Equation: Self-Focusing and Wave Collapse. New York: Springer, 1999.

[37] TANG, Z. Multi-bump bound states of nonlinear Schrödinger equations with electromagnetic fields and critical frequency. J. Differential Equations, V. 245, p. 2723-2748, 2008.

[38] TANG, Z. On the least energy solutions of nonlinear Schr"odinger equations with electromagnetic fields. Computers and Mathematics with Applications, v. 54, p. 627-637, 2007.

[39] TANG, Z. Multiplicity of standing wave solutions of nonlinear Schrödinger equations with electromagnetic fields Zhongwei Tang. Z. angew. Math. Phys., v. 59 , p. $810-833,2008$.

[40] WANG, X.; ZENG, B. On concentration of positive bound states of nonlinear Schrödinger equations with competing potential functions, SIAM J. Math. Anal., v. 28, p. 633-655, 1997.

[41] WANG, X. On concentration of positive bound states of nonlinear Schrödinger equations. Comm. Math. Phys., v. 153, n. 2, p. 229-244, 1993.

[42] WANG, Z.-Q. On the existence of multiple, single-peaked solutions for a semilinear Neumann problem. Arch. Rational Mech. Anal., v. 120, p. 375-399, 1992.

[43] WILLEM, M. Minimax theorems. Boston: Birkhäuser, 1996. 
